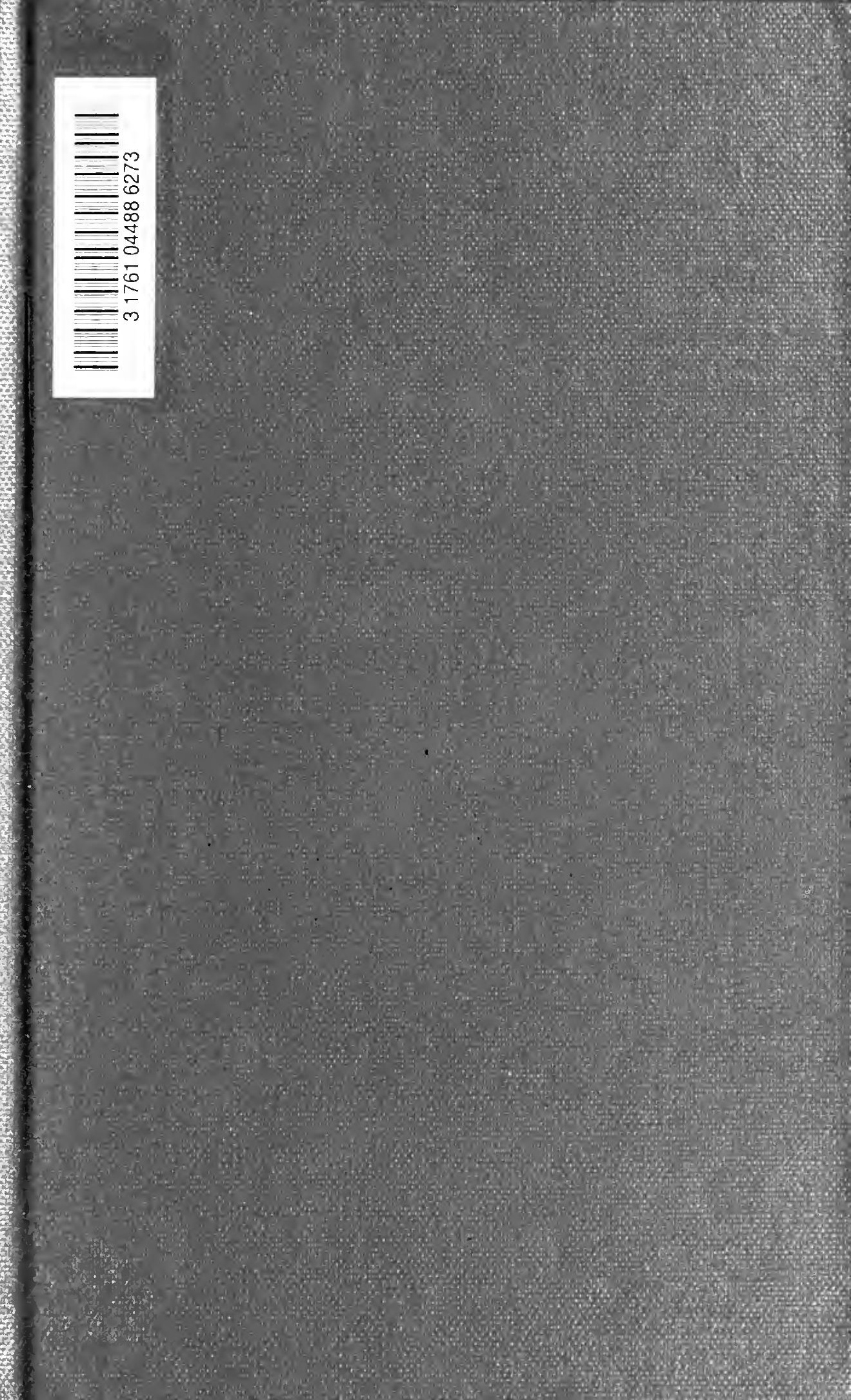


Digitized by the Internet Archive in 2007 with funding from Microsoft Corporation 



\section{PSYCHOLOGY OF THE}

\section{NORMAL AND SUBNORMAL}

BY

HENRY HERBERT GODDARD, A.M., Рh D.

Director Ohio Bureau of Juvenile Research, formerly Director Vineland Laboratory, author of "Kallikak Family," "Feeble Mindedness: its Causes and Consequences," "The Criminal Imbecile," "School Training of Defective Children."
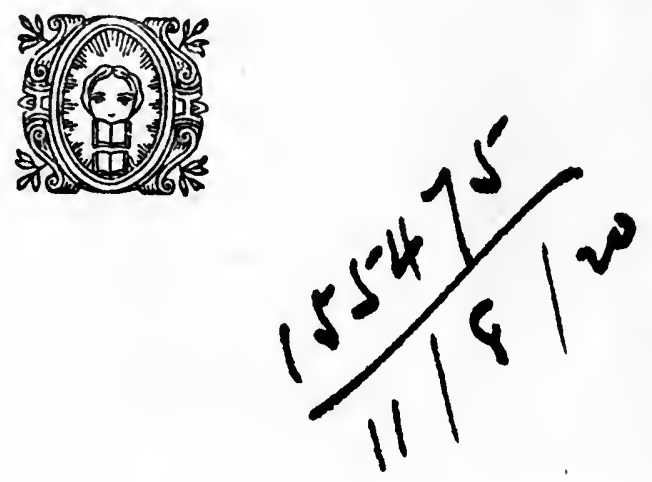

NEW YORK

DODD, MEAD AND COMPANY 
Copyright, 1919

BY DODD, MEAD AND COMPANY, INO.

PRDTTED IN U.8.A. 
THE MEMORY

OF

ALFRED BINET 



\section{PREFACE}

THE writer was once asked in what work he was engaged. When he replied, "Psychology," he was met by the remark, "Do you believe in that stuff? I used to, but I became convinced that it was all a fake." It turned out that to this man psychology meant hypnotism.

This is a fair illustration of one of the many different connotations the term psychology has in the popular mind. It is unfortunate that a science of such fundamental importance for human welfare should be so little understood.

Psychology is the science of mind; and mind determines human conduct. It would seem therefore highly desirable that the science of mind should be so formulated as to contribute to useful behaviour.

Geology is of value to civilization in that it enables man not only to understand the earth's history but also to build, to mine, to cultivate it intelligently, in short to use the earth efficiently.

Zoology enables us to know the nature of animals and to use them for our purposes.

Likewise psychology should enable us to understand the nature of mental processes and to use that knowledge to improve our behaviour and to increase our efficiency.

Psychology should enable us to control our conduct both thru our understanding of ourselves and thru an understanding of the motives and actions of others. 


\section{PREFACE}

That psychology has never reached this plane of functioning needs no proof.

Even psychologists, as a group, have not impressed their associates with any superior ability wisely to direct their own energies or to interpret the actions of others. Indeed it is to be feared that they are more generally considered as decidedly unpractical theorists, "highbrows," or even visionaries. The exceptions are probably considered exceptional men rather than exceptional psychologists. As for interpreting the actions and motives of others the case is no better. Some of those who are considered our greatest psychologists are known as the poorest judges of men.

No more surprising instance of this can be found than that afforded by the world war. For a generation we had thought the Germans were the greatest living psychologists. Yet no small cause of their downfall has been their inability to comprehend the minds of their opponents. This is shown conclusively by their methods of making war.

When one asks the reason for this failure of psychology to have practical value one is apt to get the answer that psychology is a young science. But psychology is as old as Aristotle and Plato. If someone should reply that psychology has until recently been tied to philosophy, he only proves the point. The fact that psychology remained for centuries enveloped in the mists of philosophy only shows that it was not virile enough to escape-not of enough practical value to be in demand.

It seems to us that the explanation is to be found in one of the facts of mind itself.

There seem to be two types of human beings; those [viii] 


\section{PREFACE}

who deal mainly with concrete experiences and those who chiefly use symbols.

There are mathematicians who can do wonders with figures but are not able to master the calculus.

Most "captains of industry" have "started at the bottom" and worked through all the concrete phases of the business.

Undoubtedly human development has progressed by the increased use of symbols. Symbols are time savers and energy conservers. Nevertheless symbols have their dangers. One may become so enamoured of the symbols that he forgets what they symbolize--he even gets to the point where he cares nothing for the thing symbolized. He is quite content with a clear and logical array of symbols. Now this may be very good for those who understand the symbols and enjoy their manipulation. We do not even deny that it is useful to pure science-provided it does not become a mere juggling of symbols; and, we think we should add, provided the handler of the symbols occasionally comes back to earth to see what his new symbols stand for.

But all this symbolizing takes the subject out of reach of that much larger group who must have the matter in concrete form. Moreover there will inevitably be a group who can handle the symbols fairly well but who have no notion of their significance. Who does not feel that the average graduate from a course in psychology is merely a more or less successful juggler with psychological terms!

Unfortunately for the practical use of psychology, our text books have generally been written by those who are capable of handling the symbols-with, we suspect, an 


\section{PREFACE}

occasional one written by a mere juggler with words.

If our explanation is correct, it follows that psychology must eventually be written from the practical standpoint, in terms that can be appreciated by those who belong to the second type-those who do not enjoy symbols to the extent now used in psychological works.

If the present book in any degree paves the way for this, it will serve its purpose.

If the writer has achieved any success in developing a picture of the mental processes in any way more graphic than that usually drawn it is probably due to the fact that for twelve years he has been in a position where he has been compelled by the nature of the work to look for such a picture.

In September, 1906, we opened the Vineland laboratory for the investigation of feeble-mindedness. It was not a problem of formulating a consistent theory, nor yet of applying existent theories, but rather of observing what actually occurred in the functioning of these minds.

In one particular the problem proved easy. These feeble minds were so simple that it was relatively easy to follow the various processes. It was not so easy to work it altogether into a complete picture. Indeed there are many lacunce which it has been necessary to bridge by more or less bald assumptions. In making these assumptions, however, we have constantly kept in mind one rule, namely, never to assume anything that was inconsistent with known facts.

If this book has any distinguishing characteristics we believe they are mainly two: first, the recognition of the unity of mind which we have tried to picture more consistently than is usually done; and second, in the view of the 


\section{PREFACE}

emotions which so far as we know has never been presented in a text on psychology.

Perhaps Mosso's discovery made twenty years ago was too surprising to be readily accepted in psychology or perhaps its announcement hidden away in a volume of "proceedings" did not get to the attention of the psychologists. Be that as it may, the recent work of Cannon so completely corroborates Mosso, and the view fits so well with our observations of feeble minds that we cannot doubt its correctness.

So important is this discovery that we have obtained permission to reprint Mosso's lecture. It will be found in the appendix, complete except for the description of his apparatus for which anyone interested must refer to the volume mentioned.

This view like much of the rest is destined to be much more fully elaborated as scientific experimentation is carried on.

It is hoped that this book will prove useful not only to those beginning the study of psychology in Normal Schools and Colleges but also to teachers who read for themselves, to parents who desire to understand their children, to the general reader who finds he needs to understand something of psychology in order to follow current discussions in pedagogy, sociology, feeble-mindedness, vocational guidance and allied subjects, and finally to all who wish to increase their own efficiency by understanding something of the workings of their own minds as well as the minds of their associates.

Acknowledgements are due to so many persons that the task of naming them all is hopeless. The book is essentially a product of the Vineland laboratory and all 


\section{PREFACE}

who have helped in that work have contributed to the result. This includes not only those who have been members of the laboratory staff but the Paidological Staff, the Board of Directors, Officers of the Institution and those who have helped in a financial way to make the work of the laboratory possible. My thanks and appreciation are hereby expressed to one and all.

My debt to the literature is only partly indicated by the Bibliography. The rest will be evident to the reader. Henry Herbert Goddard.

Vineland, August 1918. 


\section{CONTENTS}

\section{PART I}

CHAPTER PAGE

I Nervous System • . . . . . . . . . . .

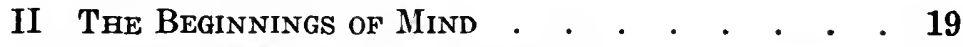

III Arrested Mental Development . . . . . . 52

IV Some Properties Inherent in the Nervous MechANISM . . . . . . . . . . . . . . . 69

[V Higher Mental Processes . . . . . . . . . 90

VI Higher Mental Processes-Continued . . . . 110

VII The Affective Side of Experience . . . . . 123

VIII Complex Emotions . . . . . . . . . . 140

IX Тнот . . . . . . . . . . . . . . 158

$\mathrm{X}$ Тнот-Continued . . . . . . . . . . 174

XI ACTION . . . . . . . . . . . . . 193

XII HABIT . . . . . . . . . . . . . . 212

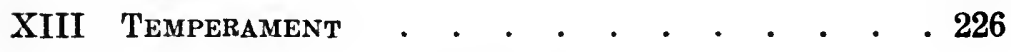

PART II

$\checkmark$ I Applications . . . . . . . . . . . . . 233

II The Determination of Mental Levels . . . . 249

III INTELLIGence ANd Will . . . . . . . . 264

IV Intelligence ANd Emotion . . . . . . . 270

V EXPERIENCE . . . . . . . . . . . . . . 277

V́l Pedagogical Applications . . . . . . . . 288

VII Moral Training . . . . . . . . . . . 310

ApPEndix

The Mechanism of the Emotions, by Professor

Angelo Mosso . . . . . . . . . . .

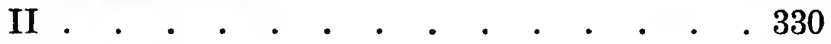

III . . . . . . . . . . . . . . 334

Bibliography • • • • • • • • . . 337

INDEX . . . . . . . . . . . . . 343 



\section{ILLUSTRATIONS}

General view of the cerebro-spinal nervous system . Facing 4

Profile of a human embryo of six weeks . . . . Facing 5

The sympathetic system . . . . . . . . . . Facing 6

Relation of the sympathetic ganglia to the spinal cord and spinal nerves . . . . . . . . . Facing 7

Profile view of a human embryo of six weeks . F Facing 8

Lateral views of human embryo at four, five and one half, and six months . . . . . . Following 8

Lateral view of adult human brain . . . . Following 8

Brain of a fifteen year old idiot . . . . . . Following 8

Section of human brain parallel to mesial surface Following 8

Section from the cortex of a two months' human

fetus . . . . . . . . . . . . Following 8

Section from the cortex of a human fetus at the beginning of the third month . . . . Following 8

Adult hrman cortical cell, showing dendrite cell body and neurite . . . . . . . . Following 8

Phylogenetic and octogenetic development of neurons . . . . . . . . . . . . Following 8

Development from birth to 110 days of a Purkinje nerve cell from the cerebellum of a white rat Following 8

Sections from the cortex of rats of different ages from birth to 20 days . . . . . . . . . Facing 9

A few of the many types of neurons in the human nervous system . . . . . . . . . . Facing 10

Section thru a convolution of the cortex of a one month old child 


\section{ILLUSTRATIONS}

Diagram showing the simplest neuron pattern from

a stimulus applied to the toe, producing sensation in cortex and returning to the muscle that moves the toe . . . . . . . . . Following 10

Central connections of the olfactory neurons . Following 10 Diagram to show the layers of cells and fibres in the gray matter of the human cerebral cortex Following 10

Human cerebral hemisphere seen from the left side . Facing 12 Some of the chief association tracts of the cerebral hemisphere . . . . . . . . . . . Facing 12

Lateral and median views of the human cerebral hemispheres . . . . . . . . . . Facing 14

Development from birth to 110 days of a Purkinje nerve cell . . . . . . . . . . . Facing 15

Sections thru brain of child at end of second week and second month of life . . . . . . Facing 16

Curves showing the thickness of the cortex in fourteen different areas of each hemisphere of the brain of Laura Bridgman in comparison with the average thickness of similar areas from ten normal brains . . . . . . . . . . Facing

Section of spinal cord showing simple reflex arc from skin to muscle . . . . . . . . Facing

Diagram of the simplest possible reflex are from epidermis thru cerebral cortex to striped muscle Facing

Showing the extra synapse in the thalamus and the pyramidal tract . . . . . . . Following

Value of synapses in reducing the number of neurons needed to associate stimuli . . . . . Facing

Diagram of telegraph relay as illustration of way nerve cells transmit energy . . . . . Facing

The probable course of impulses and the interneuronal connections in the cortex . . Following Neuron pattern slightly more complex . . Following 24 A relatively complex neuron pattern . . . . Following 24

Section from the brain illustrating a highly complex neuron pattern. Following 


\section{ILLUSTRATIONS}

Section of cortex showing the multiplicity of possi-

ble patterns .

Section of spinal cord showing simple reflex arc from skin to muscle . . . . . . . Facing 26

Diagram to illustrate possible curves of development

of intelligence from birth to 20 years . . . Facing 34

Some of the chief association tracts of the cerebral

hemisphere . . . . . . . . . . . Facing 36

Diagram to illustrate what happens in brain when

ideas are associated . . . . . . . . Facing 44

Curves showing the successive tests of several chil-

dren who are in the slowing-down stage . . Facing 62

Dorsal views of two brains . . . . . . . . Facing 64

Comparison of four types of brain cells, normal and

idiot . . . . . . . . . . . . Facing 65

Microcephalic idiot boy 22 years old . . . . . Facing 78

Brain of case pictured in Fig. 38 . . . . . . Facing 79

Curves showing development of ability as measured

by the Adaptation Board . . . . . . . Facing 104

The sympathetic system . . . . . . . . Facing 126

More important distributions of the autonomic ner-

vous system

Facing 128

MeDougall's theory of the emotions . . . . . Facing 142

Diagram of the mechanism of re-enforcement . . 151

High grade Moron, and a sample of his work . . Facing 190

Possible neuron connections in instructive, automatic

and deliberative action . . . . . . Facing 200

Curve of distribution . . . . . . . . . . . . $\quad 235$

Reproduction of Millet's Man with the Hoe . . . Facing 240

Attempts of five and six year old children to draw

a diamond . . . . . . . . . . . Facing 256

Attempts of seven and eight year old children to

draw a diamond

Facing 257

[xvii] 



\section{INTRODUCTION}

Psychology is the science of mind. It may be subdivided into human psychology and animal psychology. Human psychology may be divided into child psychology and adult psychology, and again into normal and abnormal psychology. Obviously these last overlap so that we may have normal and abnormal child psychology, and normal and abnormal adult psychology.

As long as psychology was confined to introspection for its data it was limited to normal adult human individual psychology.

In some respects this was unfortunate since it doomed the psychologist to work on the most complicated type of mind instead of the simplest.

The biologist does not seize upon the first animal or plant that comes to hand and attempt to solve his problem. Instead he seeks a plant or animal of the simplest structure that can furnish the data needed. He proceeds from the simple to the complex. The history of psychology shows the opposite procedure. The psychologist has seized upon the first material at hand. He has attempted to solve his problem by studying the adult human mind and usually a very highly developed adult. He has thus attacked the problem by the use of the most complex material possible. As a natural result, there has been much confusion, many controversies have arisen and the development of the science has been very slow.

$$
\text { [xix] }
$$




\section{INTRODUCTION}

The study of the child was a great step forward since the child mind is vastly simpler than that of the adult. But child study has had two significant limitations. The first comes from the fact that children are both normal and abnormal. But this fact was not recognized in early investigations. It is safe to say that in practically every statistical study of children $2 \%$ of the subjects were feeble-minded; and perhaps an equal per cent were abnormally bright. Such being the case the results must be contradictory and disappointing and of ten erroneous. It was like attempting to learn the normal human temperature by taking the temperature of a group of people some of whom had fever.

The second limitation comes from the fact that the normal child develops so rapidly that it is exceedingly difficult to ascertain his actual condition at any one time. It is now possible to eliminate both these difficulties. We can ascertain the normality or abnormality of the children that we study, and thus separate them into groups and study each group by itself.

In the feeble-minded we have a group of people who are not developing and consequently are more easily studied. A child arrested in his mental development at eight years has eight year mentality the rest of his life. We can therefore devote as much time as we wish to studying the actual conditions of the mind at the eight year level. The advantages of this are many. A person, who stands near-by and watches a passing train going at sixty miles an hour, can see very few of its details. If it passes at five miles an hour he can see much more; while, if it is side-tracked and stopped, the amount he can see is limited only by his time and his methods of investigation. The feeble-minded are stopped in their de[ $\mathrm{xx}$ ] 


\section{INTRODUCTION}

velopment and there are almost no limitations to the opportunities they afford for study.

In taking up such study a question at once arises as to the validity of conclusions drawn from abnormal material. Can we conclude that what we learn from these cases of arrested development gives us a true picture of normal mind? The question can be best answered by considering the general nature of the feeble-minded. First it must be noted that the feeble-minded are not cases of disease. It is true that the feeble-minded may become diseased just as do other people; but such cases must be ruled out or allowed for. The feeble-minded may become insane, they are subject to epilepsy and hydrocephalus, hemiplegias, and the like. But pure feeblemindedness is free from these and closely approximates the mental state of the normal child of the same mental age. Moreover, observation, such as a year's residence among a group of the feeble-minded permits, shows clearly that as far as they go their minds function much like the normal mind. While, as we shall see later, they are limited in the various mental processes, yet they have memories, they pay attention, they see, hear, taste and smell; they have instincts; they are glad and sorry, they exercise judgment and reason to a certain extent, show some choice and volition. They are good natured, happy, care free individuals interested in their work and their play. So that, if one is ignorant of their actual age, and their physical growth does not make the discrepancy obvious, one easily forgets that they are not normal. Indeed, visitors to an institution for the feeble-minded often think that some of the children must be the normal children of the officers of the institution.

We have just said, that if their physical growth did not [xxi] 


\section{INTRODUCTION}

make it evident that they were defective, one could forget the fact. This matter of physical growth is important. By physical growth we do not refer to deformities. A case of pure feeble-mindedness, uncomplicated by disease, shows no physical deformities that are worth considering. The group of children in the school department of any institution for the feeble-minded look like any other group of school children. In the case of the defectives of adult age, however, the body grows to adult form and stature, while the mind remains that of a child. What effect does this adult body have upon the mind? A child of six with a six year old body is normal, but a mind of six in a body of twenty must be something different. The possession of greater muscular power must produce a different mental content. For example, a larger hand with a stronger grasp must give a greater sense of power. Adult sex development with the strong instincts must modify the mental make-up to some degree. All this has to be allowed for in the final analysis; and fortunately experience indicates that it is relatively easy to make this allowance. Moreover the safety of our conclusion, that we may consider what we learn from defectives valid for normal mind, is strengthened by the fact that approximately two-thirds of such cases are cases of hereditary defect. Hence, according to the doctrine that acquired characteristics are not transmitted, we may feel sure that we do not have in these cases any gross deviation from the natural functioning of the human mind; but only a stopping of the development at an early point.

Still further, we are not limited to our study of the defectives themselves since it is always possible to turn from these to the normal child and compare the two, with the result that, having seen a trait or a process in the de- 


\section{INTRODUCTION}

fective, we are now able to see it in the normal child. Whereas not having first seen the trait in the defective we would not be able to see it in the normal child because of the complexity of his activity.

It may not be without value also to state that, from what we now know, the variation from the normal in the brains of defectives is exceedingly minute. That is to say, the gross anatomy has every appearance resembling the normal brain. Even the microscopic structure is to a large extent and in large areas apparently normal. From this we may safely conclude that up to a certain point the functioning of the brain will be normal. It is true that our knowledge of the brain of the defective is still limited, but from what we do know, we find that it is safe to reason to the normal brain; and from what we find of the mental make-up of these cases, coupled with what we know of normal brain and normal mind, we are able to draw certain conclusions and make certain analogies and inferences that tell us much about the condition of the feeble-minded. With these precautions we shall proceed in the following chapters to draw a picture of the workings of the human mind, taking into consideration at each step such facts as we have been clearly able to observe in these cases of arrested mental development.

Since a nervous system conditions the mental processes and it is easier to understand an abstract concept if we understand the concrete thing from which it was abstracted, we shall devote the first two chapters to a working plan of the nervous mechanism, using such facts as are generally accepted by neurologists and supplementing these by such hypotheses as are necessary to complete the picture and at the same time are not contradicted by any known facts. 


\section{INTRODUCTION}

It will be well for the student always to differentiate very carefully between what is known fact and what is an hypothesis assumed for the sake of completing the picture. Joseph Cook used to say that he did not like to call himself a seeker after truth because "truth" too often means one's own opinion. But he thought it was much safer to be a seeker after clearness. In the following pages we have sought for clearness with the underlying thought that while there is no expectation that our formulation is the final truth, yet if we violate no known facts, our formulation will be helpful in proportion as it is clear. 


\section{PART I}

\section{PSYCHOLOGY OF THE}

NORMAL AND SUBNORMAL 



\section{PSYCHOLOGY OF THE NORMAL AND SUBNORMAL}

\section{CHAPTER I \\ NERVOUS SYSTEM}

THE human nervous system is the most elaborate and complicated structure in the entire body. It consists of two main parts, the cerebro-spinal and the sympathetic. The latter is sometimes called the vegetative and more recently the autonomous, nervous system.

The cerebro-spinal system is again sub-divided for convenience into the central nervous system composed of the brain and spinal cord, and the peripheral system composed of the nerves extending to the surface of the body and modified as special sense organs or as the nerve endings attached to muscles.

The central nervous system, and especially the brain, has usually been considered the main and almost the only organ of mind. More recent studies, however, have shown the importance of the sympathetic system for an understanding of certain phases of mind. This will be discussed in its place.

Of the central nervous system the spinal cord is relatively simple. It may be thot of as a tube of nervous tissue, enclosed in and extending about two-thirds the length of, the spinal column. The wall of this tube is relatively very thick and the canal very small. From the anterior side of each segment of this cord, corresponding 


\section{PSYCHOLOGY OF THE NORMAL AND SUBNORMAL}

with each vertebra in the spinal column, nerves pass out to the muscles. At the posterior side of each segment enter nerves from the sensory organs at the surface of the body-the skin, tendons, etc. The nerves, passing up and down the cord, constitute the principal part of the cord itself.

The brain is so enormously complicated that it has taken years for scientists to understand as much as is now known about its structure. The problem is much simplified if we understand that the brain is the modified upper end of the spinal cord contained within the skull. The complication arises from the fact that within the skull this long, comparatively simple spinal cord has become bent up, its different parts have grown at different rates, and the whole has crowded itself into the generally spherical shape of the skull cavity. The result is the solid mass that we recognize as the brain.

The number and direction of the flexures that have taken place may be more easily understood if one thinks of a person in a closely crouching position. The foot will represent the spinal cord as it enters the skull; the ankle is the first bend, the knee the second and the hip the third. The under side of the thigh represents the part that develops into the cerebellum. Think now of the head which represents the cerebrum growing back and covering the body as far as the hips and one has a convenient, if crude; picture of the growth of the encephalon - the part within the head.

The arrangement of nerves going out from the cord and coming in from the surface may still be made out within the skull, tho because of the irregular growth and crowded condition their course is very much complicated and has been very difficult to trace. This part of the 

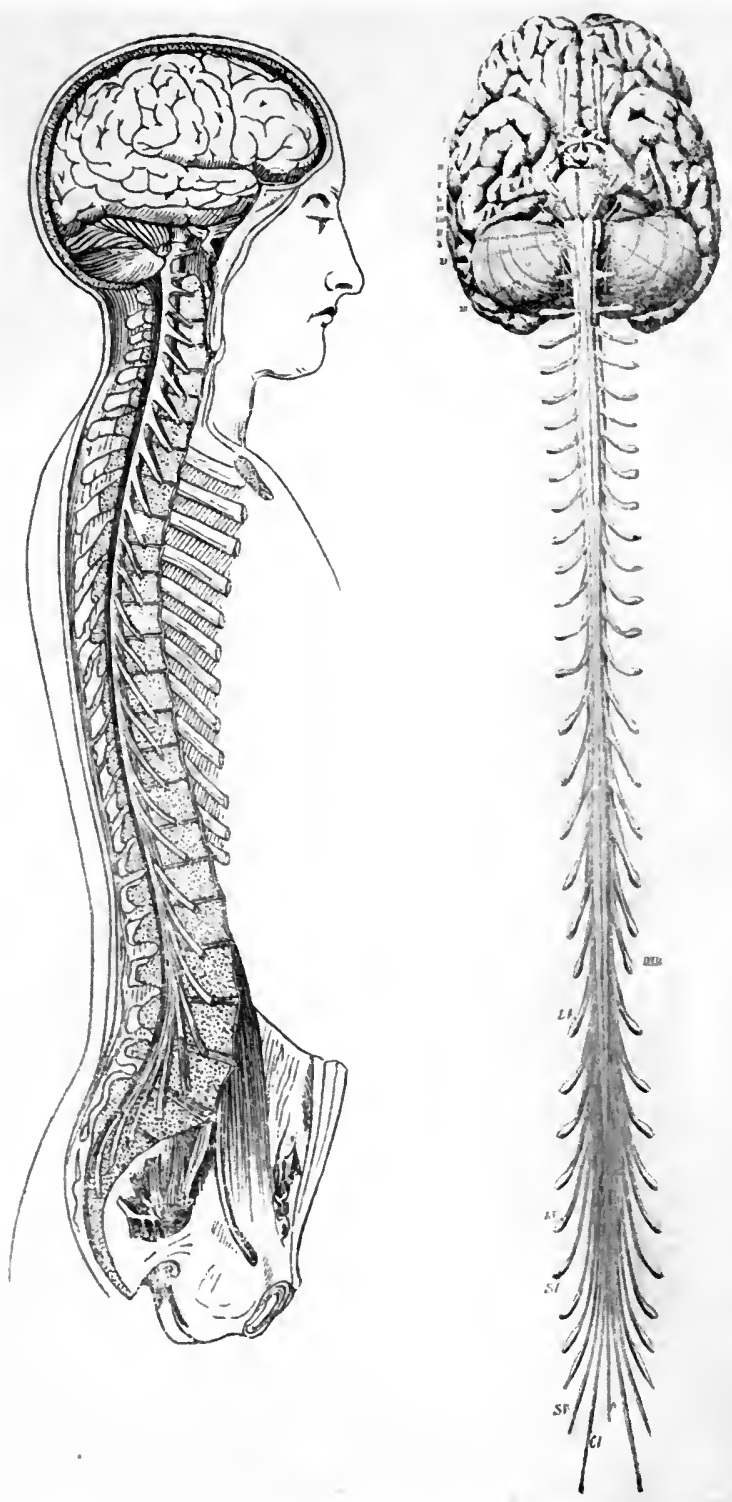

Fig. 1. A, General view of the cerebro-spinal nervous system slowing location in the head and spinal column.

Fig. 1. B, the same removed. Seen from the front with the brain (the enceplialon) turned back so as to show its under side. The paired branches are the spinal nerves. The slight swelling at the end of each indicates the spinal ganglion. At these points the nerves have been severed from the peripheral nervous system. 


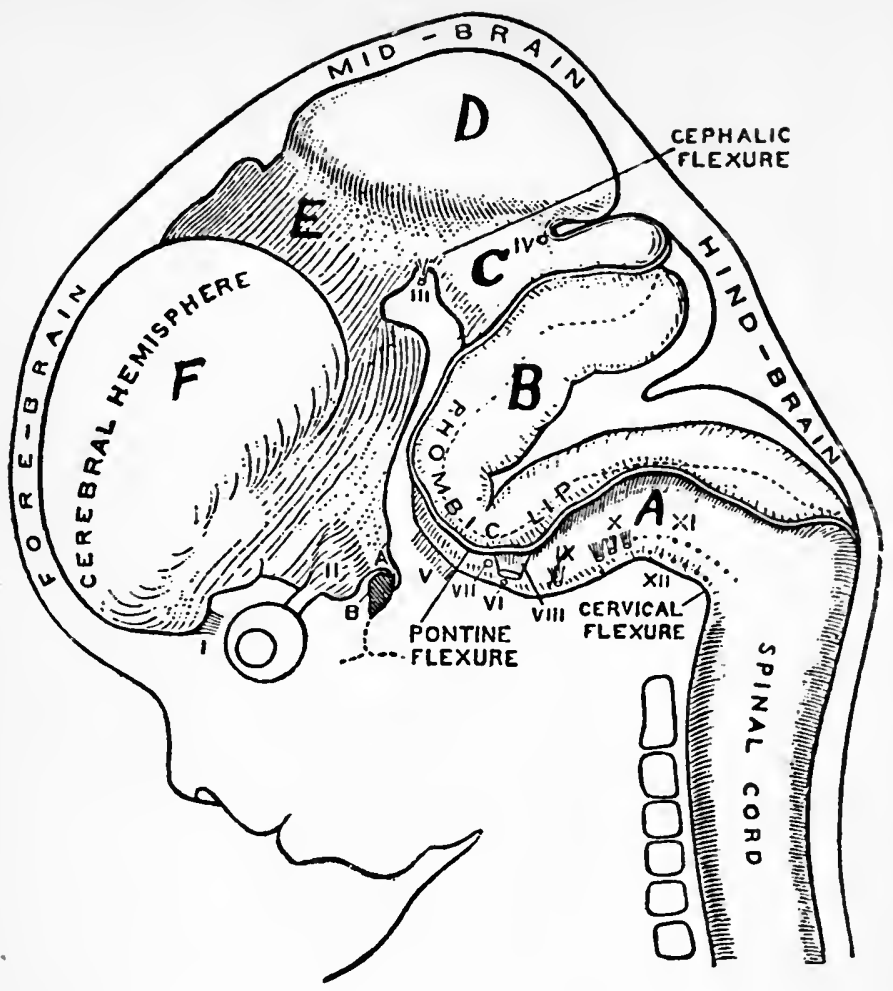

Fig. 2. Profile view of a human embryo of 6 weeks. As growth adrances the cerebellum will appear as an outgrowth at B; and F, cerebrum (sometimes called the pallium mantle) will rapidly extend until it envelops all that is here shown except a part of $\mathrm{A}$ and the cerebellum, which tho partly covered by the cerebrum is nevertheless distinctly visible in such a view as Fig. 1. A and B.

From Dunlap 
primitive spinal cord which is situated within the skull is called the brain, or often the encephalon. It comprises three main divisions, counting from the point where the cord enters the skull: the medulla oblongata, the cerebellum and the cerebrum.

Fig. 1 shows a picture of the central nervous system, that is, the spinal cord with the encephalon. A pictures it as it is located in the body. B the same removed. The position of the spinal nerves is shown and the points at which the peripheral nerves have been cut off.

Fig. 2 shows the encephalon while still in a primitive condition; it is possible to trace the several turnings and to note where the medulla, the cerebellum and the cerebrum develop. It will be noted in this figure that at this period the cerebrum is relatively small and located at the very extreme front end of the cord; and also that this outgrowth takes place on the two sides of the anterior end of the cord. From this it results that the complete cerebrum is in two parts known as the cerebral hemispheres. As development proceeds, the various parts become very much more closely crowded together with the result that in the adult brain it is impossible to trace the curves and bends of the cord.

The cerebellum develops from the part marked B in Fig. 2, and covers the medulla. The cerebrum grows so much faster and becomes so much larger than the rest of the encephalon that it grows over everything that is seen in Fig. 2, as far back as the part marked B, from which the cerebellum develops, and even covers a large part of that organ. We shall presently discuss this growth of the cerebrum and picture its development at various stages, both its external shape and its size and internal structure. 


\section{PSYCHOLOGY OF THE NORMAL AND SUBNORMAL}

The Sympathetic System.-Fig. 3, A and B, gives a general view of the arrangement of the nerves of the sympathetic system. This also is a very elaborate system in that it extends to all the organs within the abdominal cavity as well as to the involuntary muscles, and so extensive is it in each organ, that the statement has been made that were all the other tissue of each organ removed leaving only the sympathetic nerves, that they alone would preserve completely the shape of the organ. This will give some idea of its extent.

The cerebro-spinal system and the sympathetic are connected at a series of points. The nerves that pass in and out from the spinal cord at each vertebra, as already described, send off two branches each to what are known as the sympathetic ganglia. One of these branches is evidently outgoing and the other incoming. Fig. $4 \mathrm{~A}$ shows this connecton. Fig. $4 \mathrm{~B}$ shows it in detail. There is no other known connection between the two systems. We shall now turn to consider more in detail the growth and structure of the cerebrum.

Just as the body as a whole grows from about 6 pounds weight at birth to say 140 pounds at age of twenty and from a helpless weakling to a man of strength, so the brain grows from 360 grams (12 ounces) average weight at birth to about 1400 grams (3 lbs.) at age of twenty. ${ }^{1}$

Coincident with this growth of brain is a growth of mind from infant to adult intelligence.

The development of mind is dependent upon the growth of the brain.

1 Professor Marchand, of Marburg, in a study of 1169 cases, finds the average weight of the brain at birth of a male child is 360 grams and of a female child 353 grams. The maximum brain weight is usually attained about the twentieth year, when the male's average is about 1400 grams. The female maximum is usually reached about the seventeenth year, when the average is 1275 grams. 

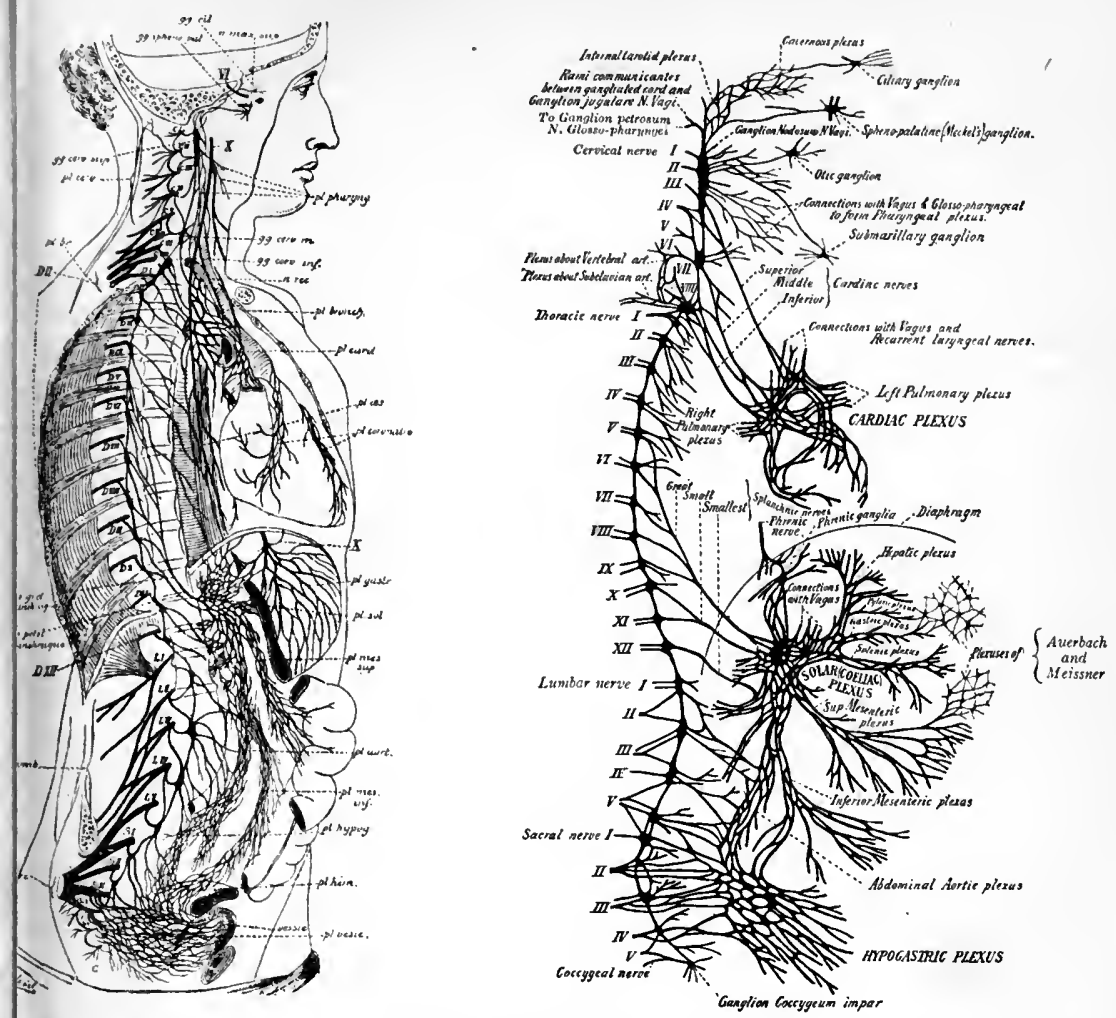

Fig. 3. A, the sympathetic system showing its location in the body.

Fig. 3. B, the same removed. The black knots in the chain are the sympathetic ganglia: the paired lines extending to the left from each ganglion are the rami communicantes or connecting branches that join the sympathetic system to the cerebro-spinal. This chain is just outside the spinal column.

From Van Gehuchten after Schwabbe 


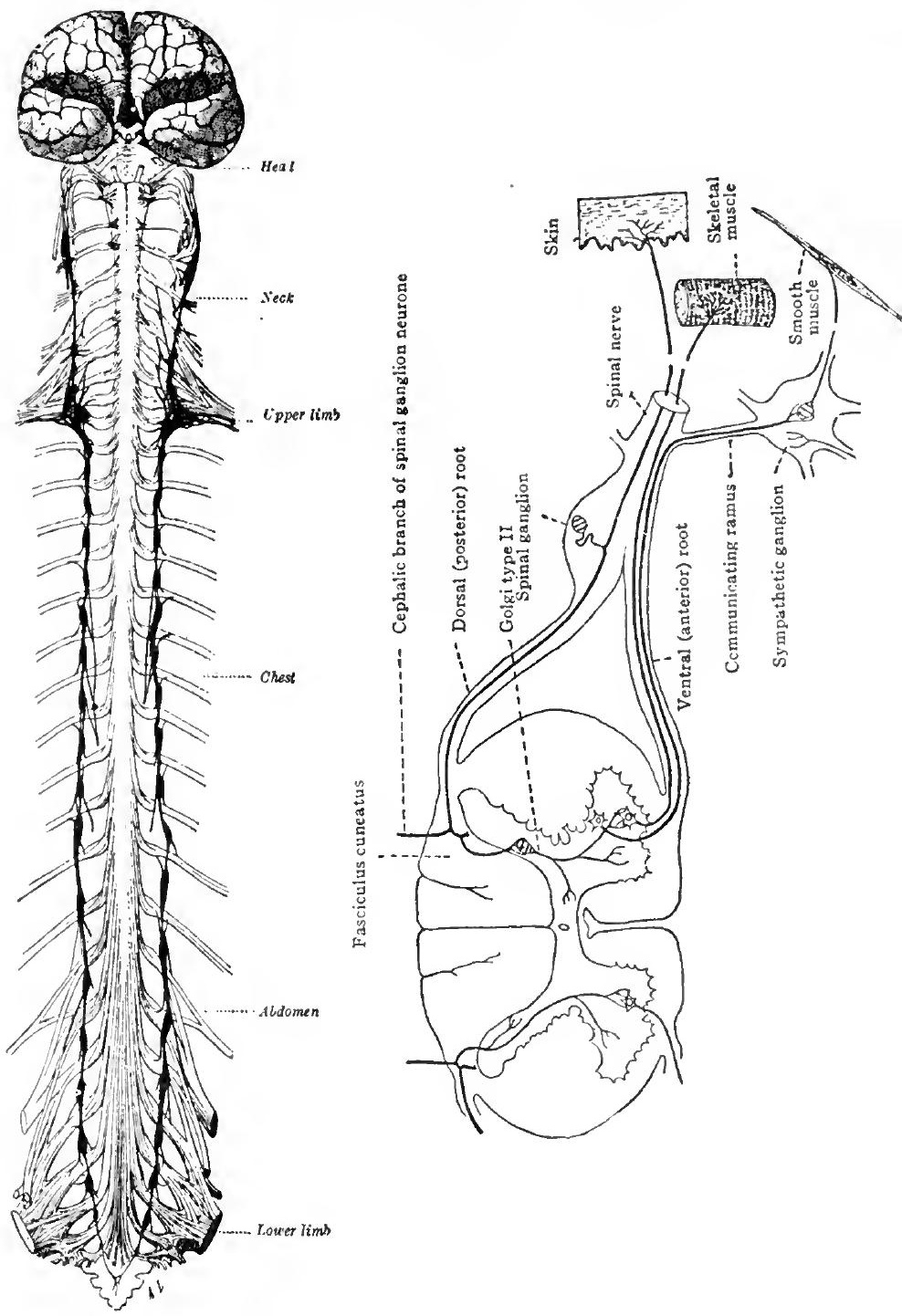

Fig. 4. A, detail showing the relation of the chain of sympathetie gan glia (black) to the spinal cord and spinal nerves. At the top, th brain seen from the front.

From Morat

Fig. 4. B, detail. Cross section at a segment of the spinal cord, show ing spinal nerves and spinal ganglion and sympathetic ganglion. 
Fig. 2 shows the brain of the human embryo at 6 weeks. We pointed out (p. 3) that the part marked cerebral hemisphere would, as development proceeded, largely cover the other parts here shown. At 16 weeks this has actually taken place. Fig. $6 \mathrm{~A}$ shows this stage, while B, C, \& D show the further growth up to adult life. An idea of the amount of growth can be had from comparing these cuts, since each shows the actual size at the time given. From the stage shown in Fig. $6 \mathrm{~A}$, the brain and especially the cerebrum grows faster than the skull which surrounds it. The result is that the cerebrum "folds in" making wrinkles or as they are technically called convolutions or gyri (singular gyrus). The space between two gyri is called a fissure or sulcus (plural sulci).

Fig. $6 \mathrm{~B} \mathrm{C} \mathrm{D}$, shows the increasing development of these convolutions. These figures show only the outside convolutions: there are also mesial surfaces where the two hemispheres meet. These mesial surfaces have their convolutions the same as the outside surfaces. These mesial surfaces can be seen in figure 7-bottom rightwhere the hemispheres have been cut through the median line and laid out flat. If one imagines the two parts here shown, hinged at their adjacent edges and folded together he will understand the true relationship.

In Fig. 8 can be seen the cerebrum extending over the cerebellum, the attachment of both to the "brain stem" (the end of the primitive spinal cord), and the layer of gray matter known as the cortex that constitutes the outer layer of the cerebrum and cerebellum.

The brain grows not only in bulk; as the weights given on page 6 would indicate, but also in complexity of structure and this is by far the most important fact.

Turning now to the internal structure and development 


\section{PSYCHOLOGY OF THE NORMAL AND SUBNORMAL}

of the brain we find conditions and changes no less striking than the change in external appearance. The brain is made up principally of two kinds of tissue. The nerve tissue comprises an enormous number of individual cells called neurons. The child at birth has ten thousand million $(10,000,000,000)$ brain cells or neurons, which number is never increased throughout the life of the individual. The growth and increase is in size, not in number. Since the brain is relatively well developed at birth, we must go back to an earlier period to see these cells in their simplest form. Fig. 9 shows a section from a two months old fetal brain. The nerve cells appear in ordinary cell form, microscopic, globular masses each with its nucleus and nucleolus. Fig 10 shows a section of the brain of a three months fetus. Here is seen the beginning of the development of these cells into the specialized neuron form. Fig. 11 shows the fully developed cortical brain cell of the adult. Fig. 12 (lower part) shows the various stages of brain development of cells from their earliest appearance in the embryo to full maturity.

Fig. 12 (upper part) shows the development of the same type of cell in the animal series, from which it will be seen that the human adult has the most elaborately developed cortical cell of all animals.

Not all the original cells develop into the same type. There are many forms. Fig. $13 \mathrm{~A}$ shows the development of another type, the Purkinje cell, found in the cerebellum. It is characterized, as will be seen, by the enormous branching of its dendritic process. B shows the development of the cortex in thickness and in complexity.

A nerve cell or neuron is composed of three parts, the cell body, the dendrite or tree-like portion and the neurite. See Fig. 11. In the cortical cell shown and in the 


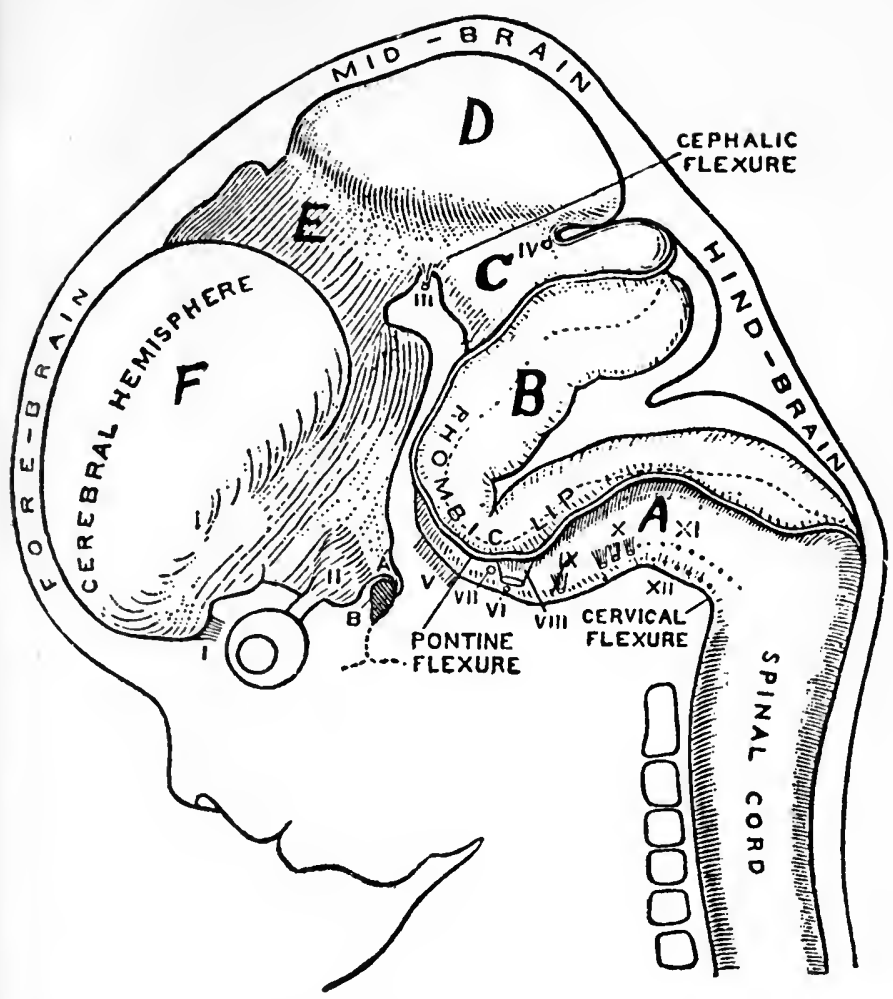

Fig. 5. Profile view of a human embryo of 6 weeks. As growth advances the cerelellum will appear as an outgrowth at $\mathrm{B}$; and $\mathrm{F}$, cerebrum (sometimes called the pallium mantle! will rapidly extend until it envelops all that is here shown exrept a part of $\mathrm{A}$ and the cerehellum, which tho partly covered by the cerebrum is nevertheless distinetly visible in sucli a view as Fig. 1. $A$ and $B$. 

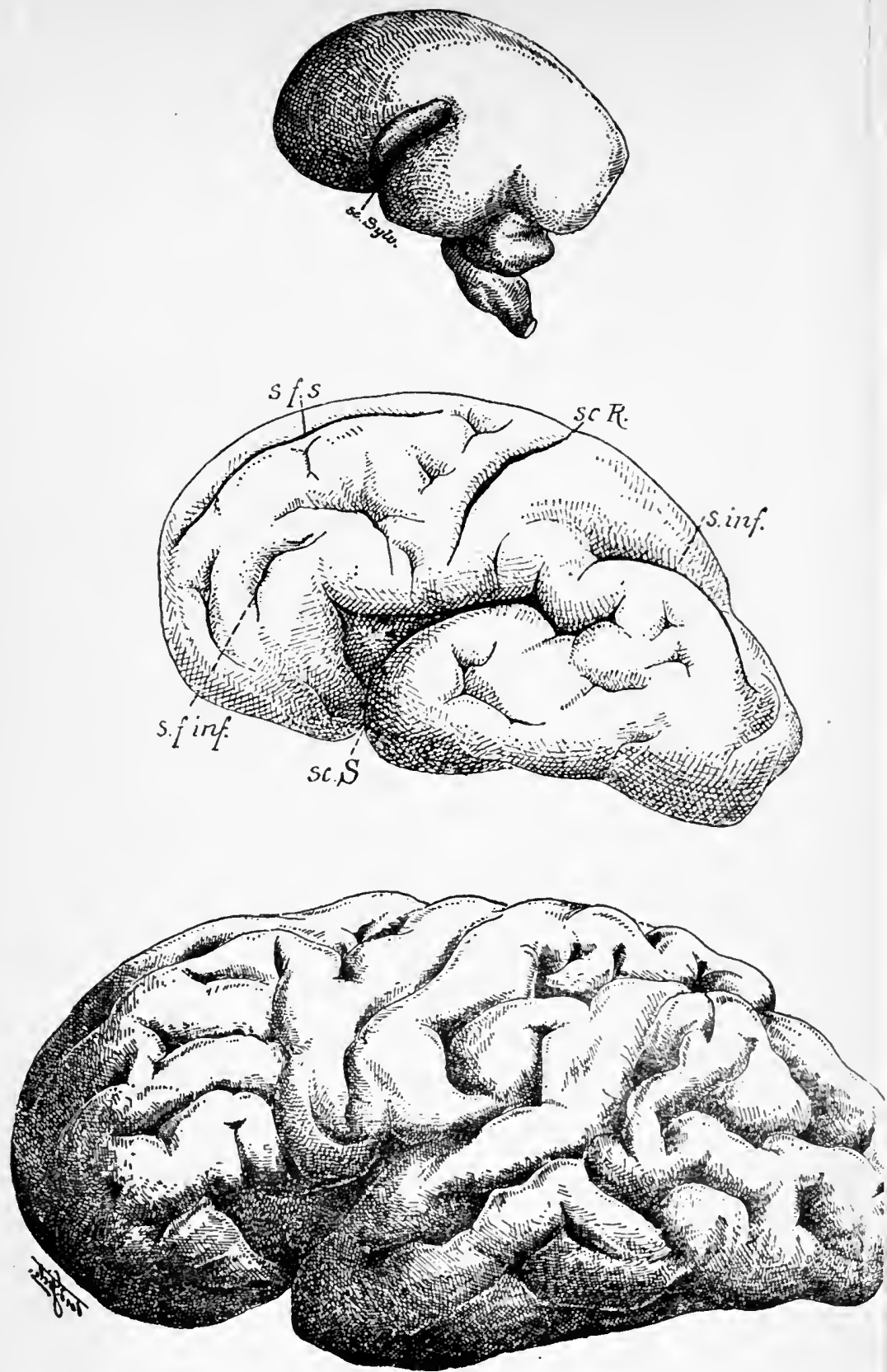

Fig. 6. A, lateral view of human embryo at about 4 months. Natural siz

Fig. 6. B, similar view of brain of human embryo at about $5 \frac{1}{2}$ month Natural size.

Fig. 6. C, similar view of ehild born at 7 months. Natural size. Note th rapid growth and increase of the number of convolutions and gyri. 


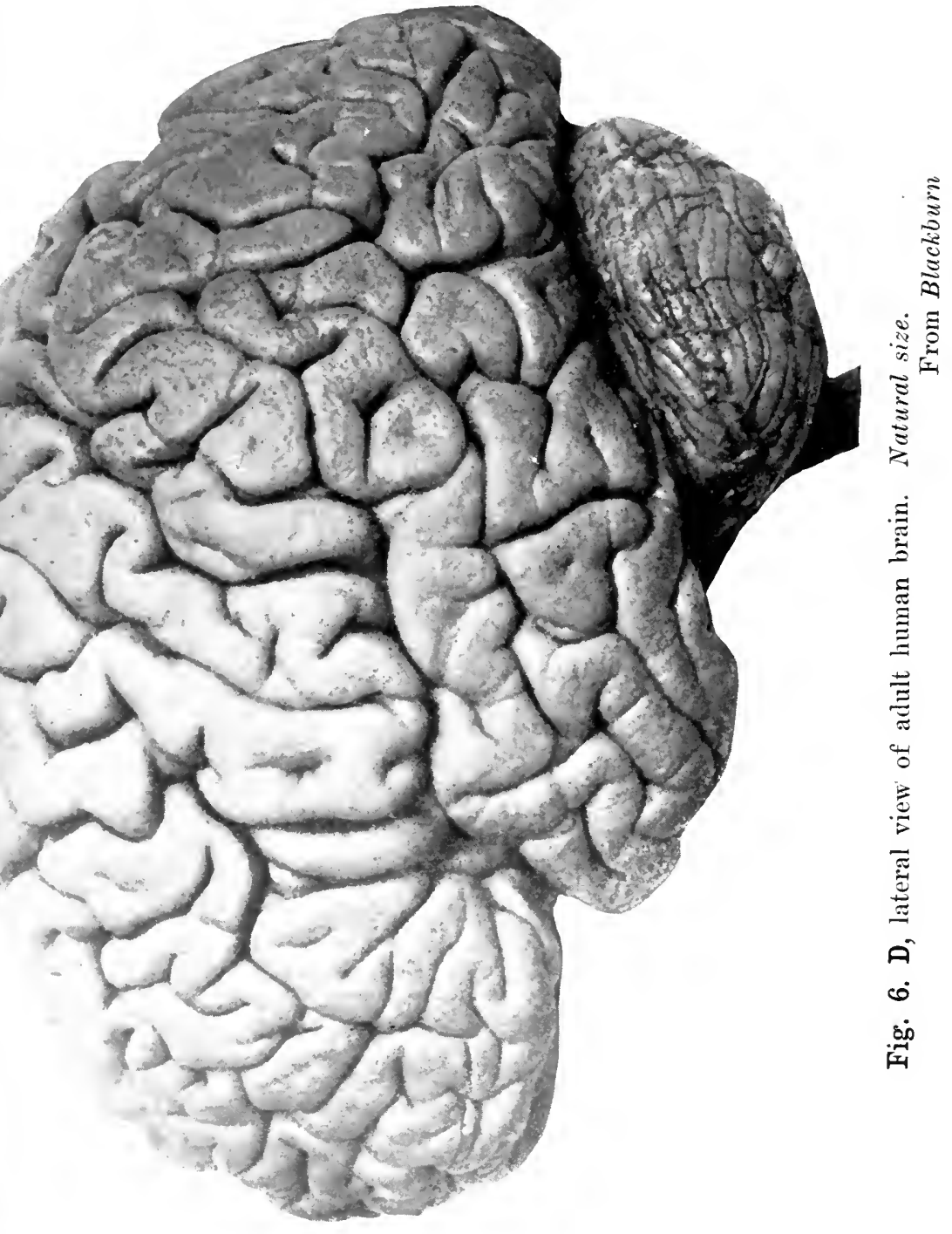




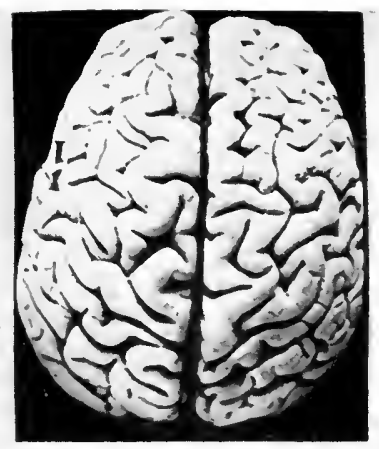

UPPER SURFACE OF CEREBRUM

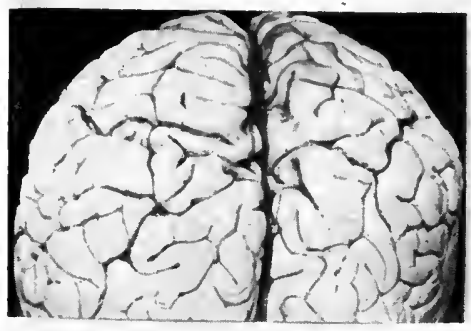

POSTERIOR END

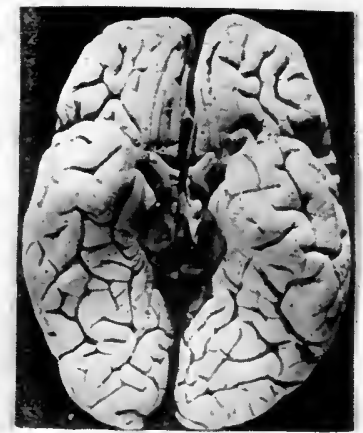

UNDER SURFACE OF CEREBRUM

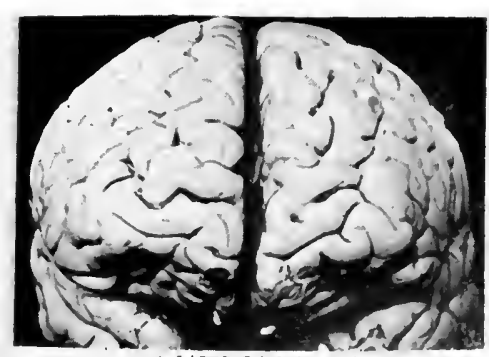

ANTERIOREND

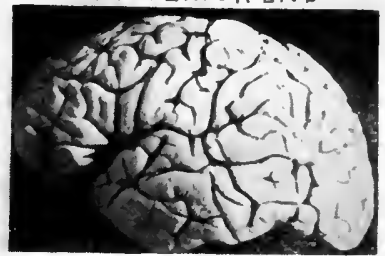

EXTERIOR SURFACE LEFT

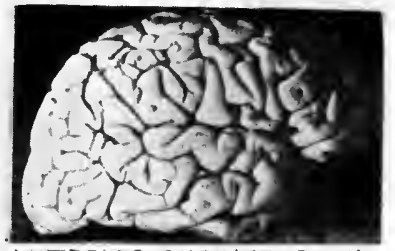

EXTERIOR SURFACE RIGHT

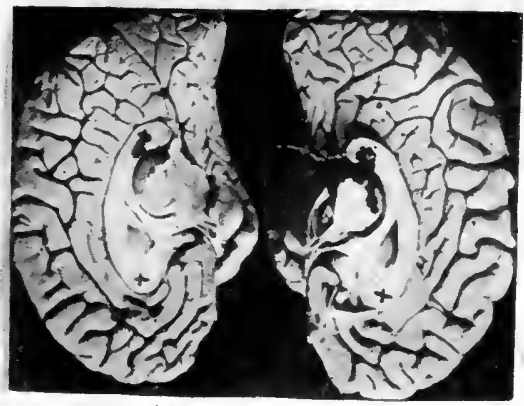

MESIAL SURFACES

LEFT HEMISPHERE RIGHT HEMISPHERE

Fig. 7. The brain of a 15 year old idiot viewed from every position-abore, below, right and left side, anterior and posterior ends, and mesial surface. For the latter the two hemispheres latre been cut apart. 


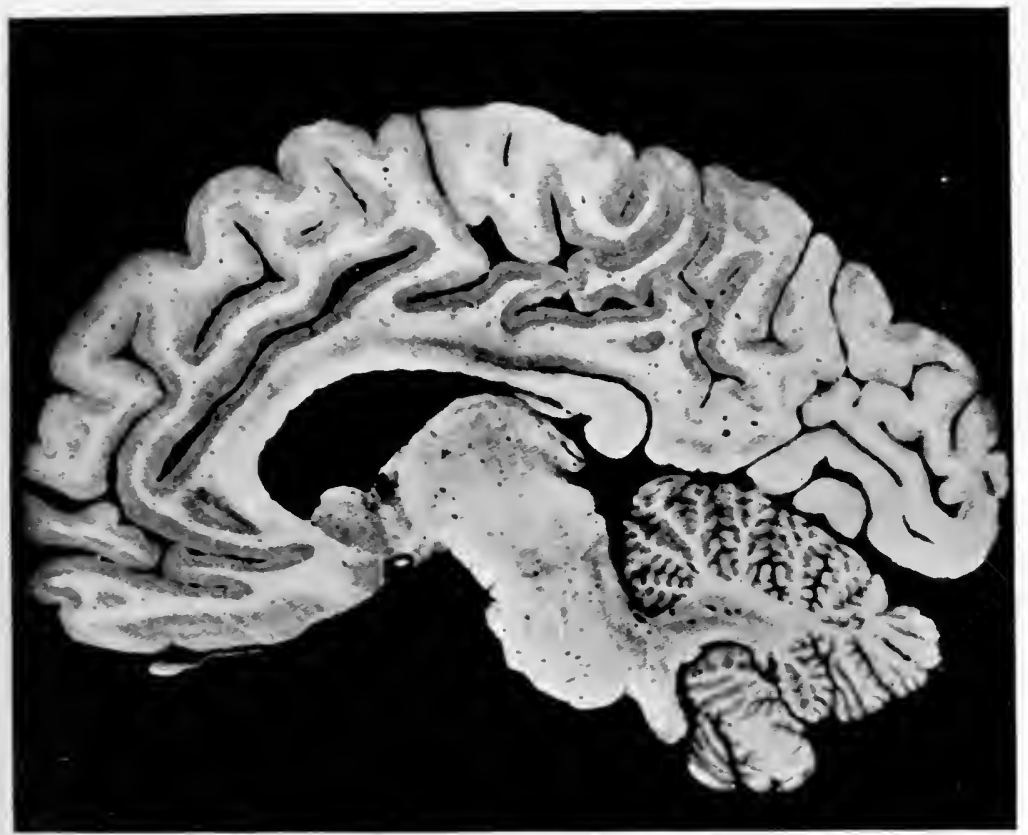

Fig. 8. Section of human brain parallel to mesial surface and about $1 / 4$ inch to the right of it. The section not only cuts across the convolutions of the external surface but it also cuts off the fissures from the mesial surface. Bounding these convolutions and fissures is the cortex (the gray band). The cerebellum is seen at the lower right. The central mass from: which the cerebellum seems to grow is the brain stem-the upper end of the primitive spinal cord. Note that the cerebrum is attached to it only at the very front (left) end. Section is about $2 \%$ natural size. Made from a preparation by the author. 


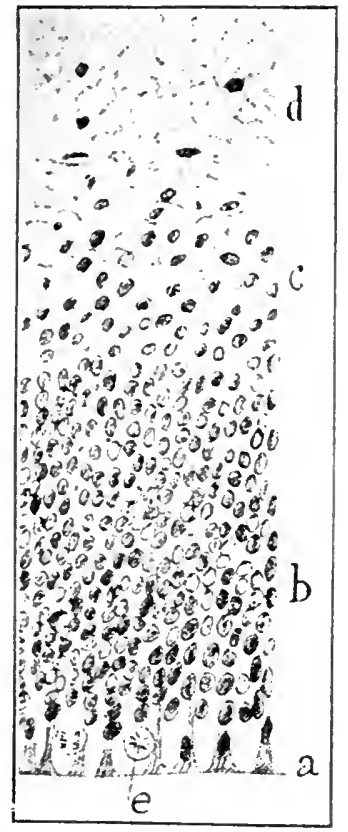

Fig. 9. Section from the cortex of a two months' human fetus. Figs. 9 and 10 from Bailey and Miller after His. Cajal

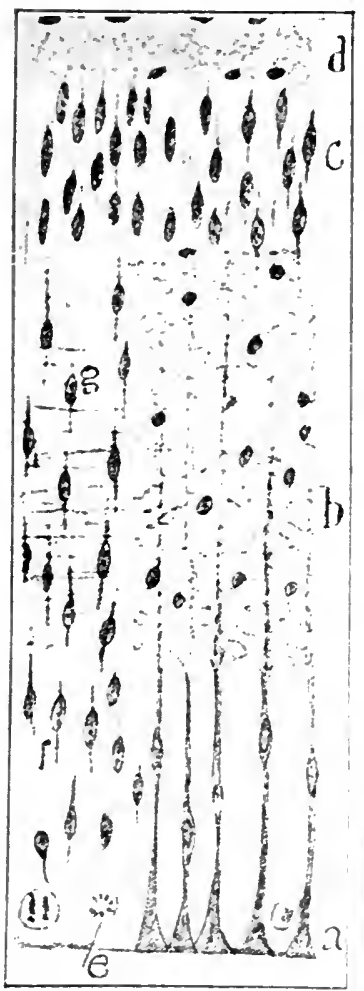

Fig. 10. Section from the corte: a human fetus at the begin of the third montl. Comi with Fig. 9 and note the r: development of the nerve $c$ 


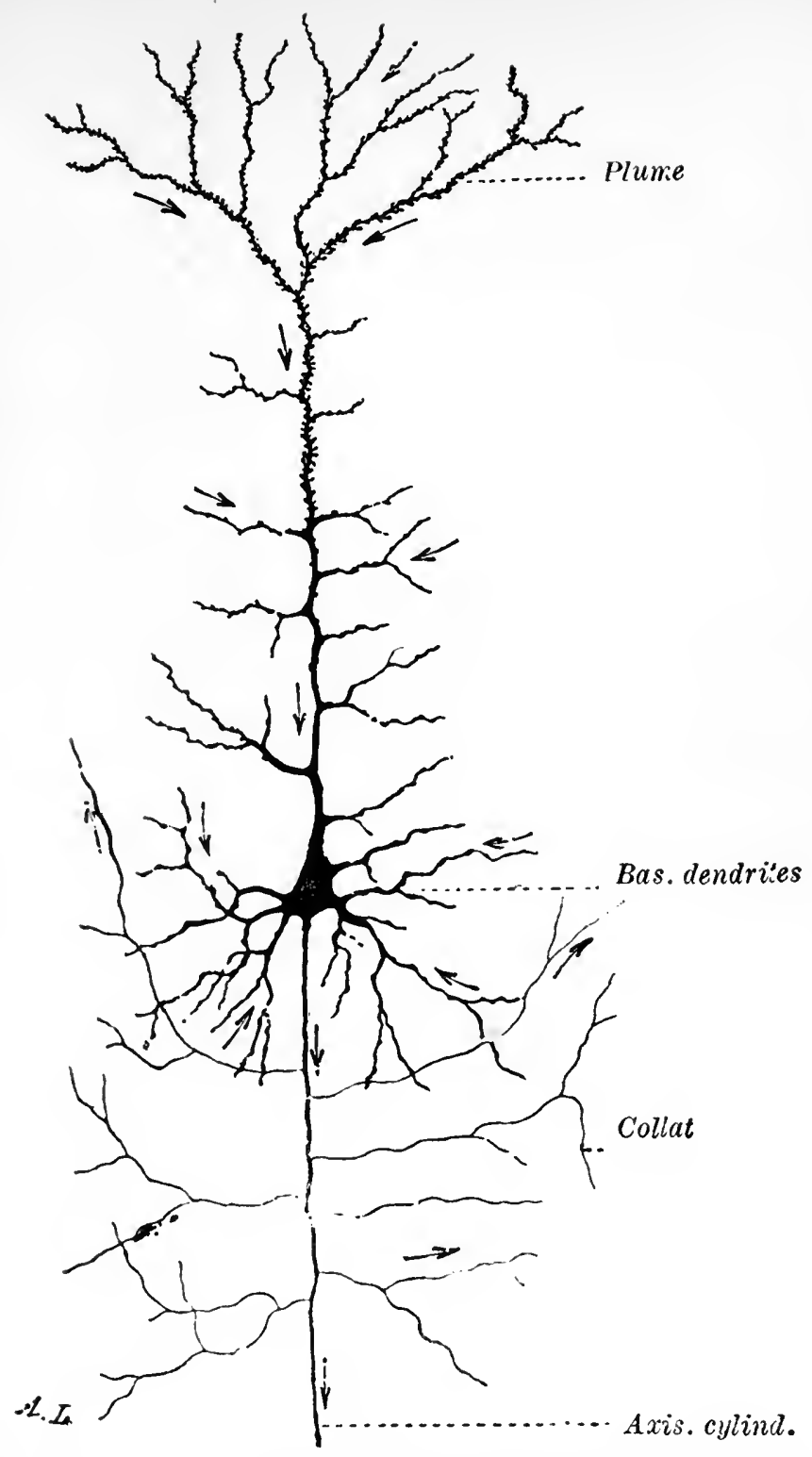

Fig. 11. Adult human cortical cell, slowing dendrite (tree like portion) cell body and neurite (fine vertical line and its branches the "collaterals"). Arrows show the direction of the flow of nervous energy.

From Morat 


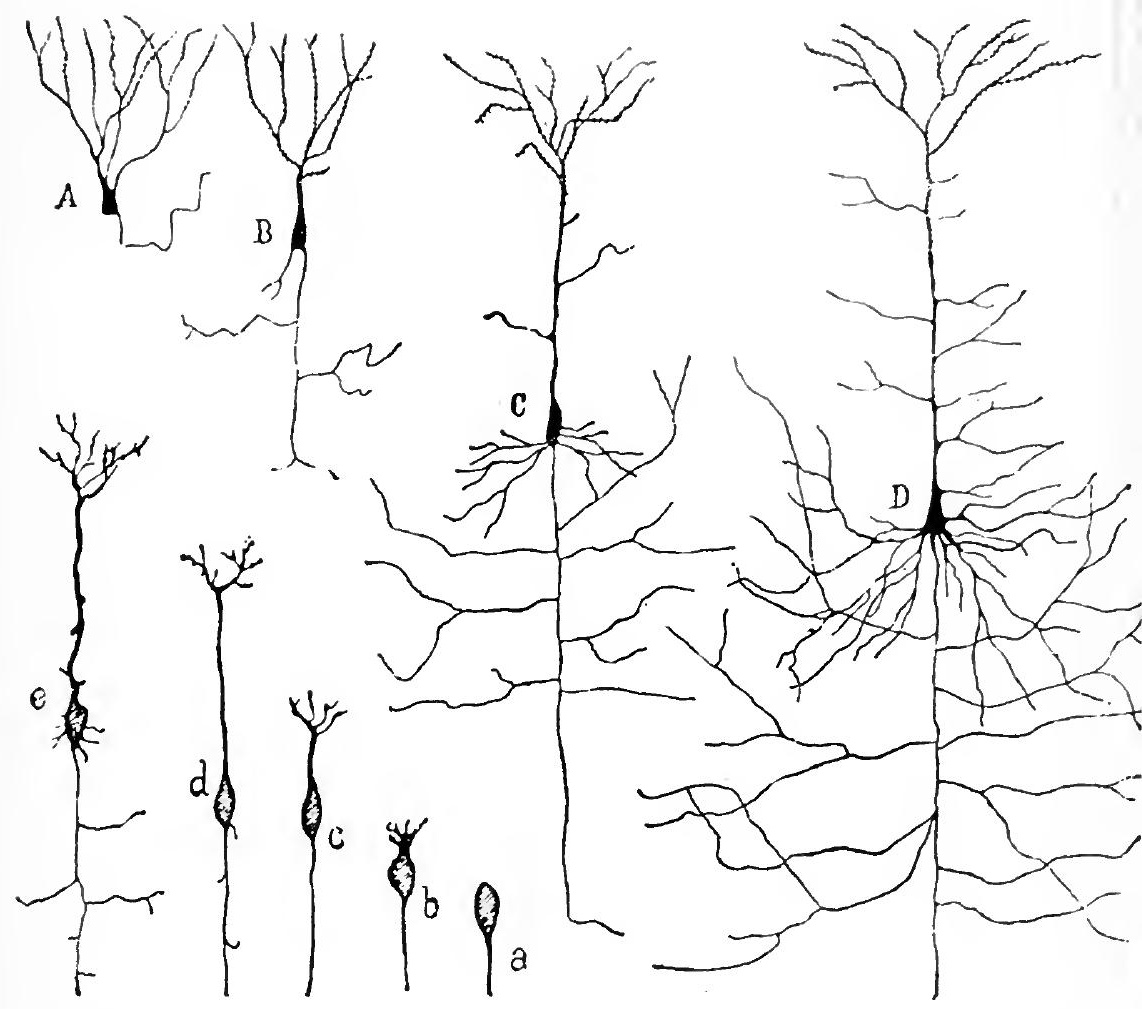

Fig. 12. Phylogenetic and untogenetic derelopment of neurons with long axones from pyramidal cells of eerebral cortex. Upper series represents phylogenetic development of these cells: $A$, in frog; B, in newt; $C$, rat; D, man. The lower series shows the ontogenetic development of the neuroblasts of those cells in five successive stages a, b, c, d, e.

From Cajal 


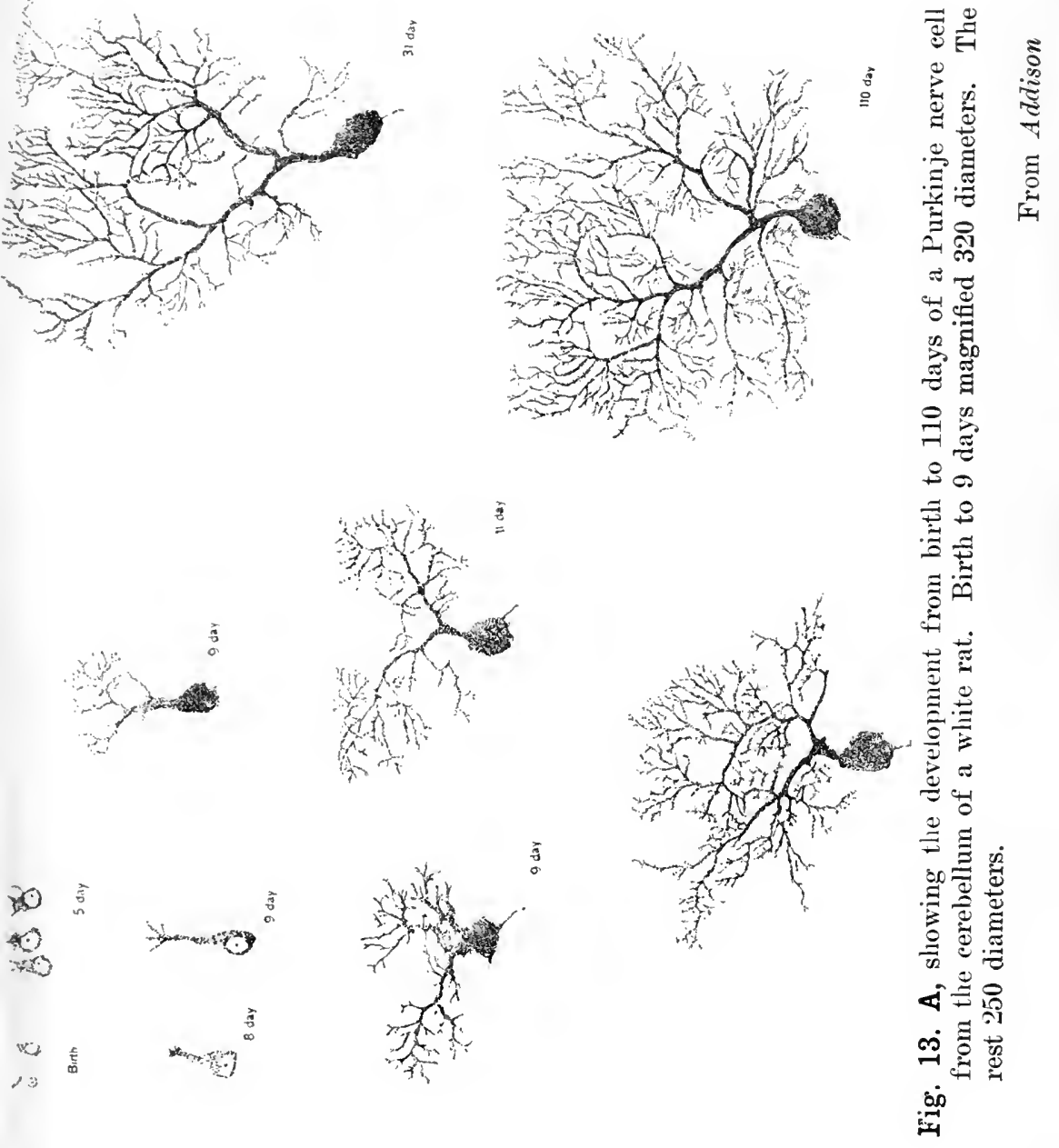


$3-1+5$

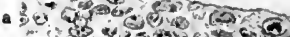
con colo s a b 20 ind a Q

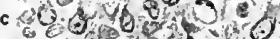
- o 6 कo (-) $\cos \theta+2$

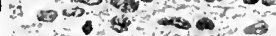

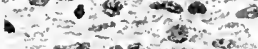

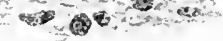

Birth

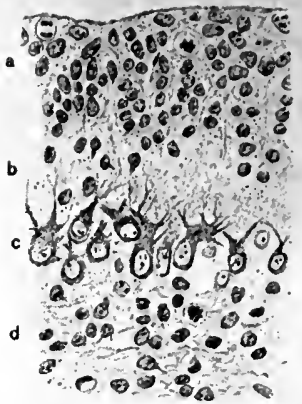

3 day

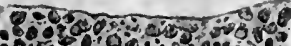

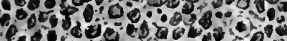

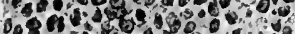

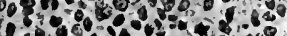
- $090^{\circ} 0^{\circ}-6^{\circ}$

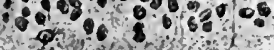

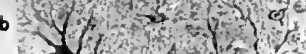

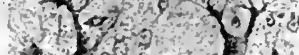

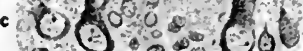

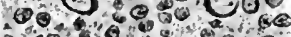

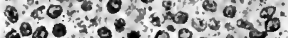

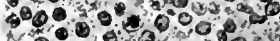
(j) d 0.400009090000

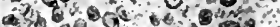
क. $=60.0 \times 0.090$ $0,0,00 \%$

8 day

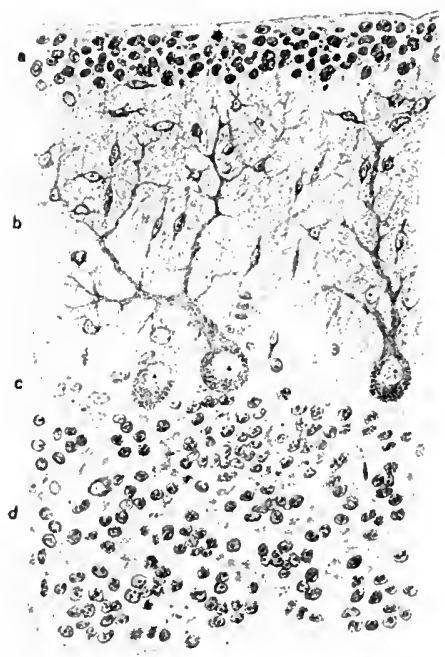

14 day

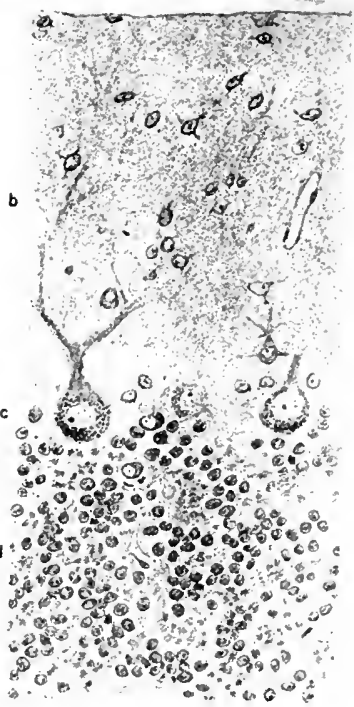

20 dey

Fig. 13. B, sections from the cortex of rats of different ages from birth to 20 days, showing rapidity of growth in thickness of cortex and of the cells.

From Addison. 


\section{NEURITES AND DENDRITES}

Purkinje cell the dendrite and the neurite have a distinctly different appearance, but in many types no such difference is apparent. Different views have been held as to the functions of these two, but at present there is no accepted difference except that the dendrite is the part that receives the energy and the neurite passes it onl. The cell body is approximately the same in all cells but the dendrites and neurites take many different forms. Whereas the cell body and the cross section of the neurite or dendrite are microscopic, the length of the neurites or dendrites may be relatively great. For example a sensory neuron may have its dendrite in the toe and the end of its neurite in the neck (see Fig. 16) a cortical cell (motor) has its dendrite and cell body in the cortex but its neurite may extend to the lowest segment of the spinal cord.

We must think of the ten thousand million neurons as divided into many groups. Some of the many different types are shown in Fig. 14. The tracing of these different groups and the inter-relations of their fibres and the functions of each constitutes a study by itself, the science of neurology. For our present purpose we need only a very crude and simple statement of the general plan. Those who aspire to a more thoro understanding of the system (and it is a most commendable aspiration) are referred to special books on the subject. Perhaps the most convenient for the beginner is Herrick (23), where will also be found abundant references to larger works.

Each neuron generates and transmits nerve energy, called neurokyme. The whole nerve system is perhaps best understood by likening it to an elaborate telephone system, where the cell body is the battery and the neu- 
rites and dendrites are the wires. The neurites and dendrites are often called nerve processes or nerve fibres and, whether long or short, when fully developed they generally end in many branches, each branch being in close relation to the branches of other neurites or dendrites. This point of contact or proximity of the endings of the branches of different neurons is called the synapse. By this arrangement, each neuron is in possible connection with a large number of other neurons just as in the telephone system one may call in over his line and become connected with any other line in the whole system.

Fig. 15 gives a good idea of the way these neurons are placed and the possibilities of inter-communication from one neuron to any one of many others. To make the matter still clearer we give in Fig. 16 the neuron connections for a simple sensori-motor experience. Something presses against my toe and I immediately move it to escape the pressure. The object touching my toe stimulates the end of the dendrite that is just under the skin. That dendrite extends from that point to a point just outside the spinal cord where the leg joins the trunk. Here will be found the cell body in what is known as a sensory ganglion or spinal ganglion. The neurite from that cell body passes into the spinal cord and up the cord to its very top in the neck. Here it ends in many branches. One of these is close to the very short dendrite of another neuron whose neurite extends on to the very top of the brain, just about at the top-most point of the head, in the cortex. Here it too ends in many branches, one of which is in contact with a very short dendrite, whose cell body lies near by, but whose neurite extends down thru the brain and thru the spinal cord to a point on the front side of the cord opposite the sensory ganglion 


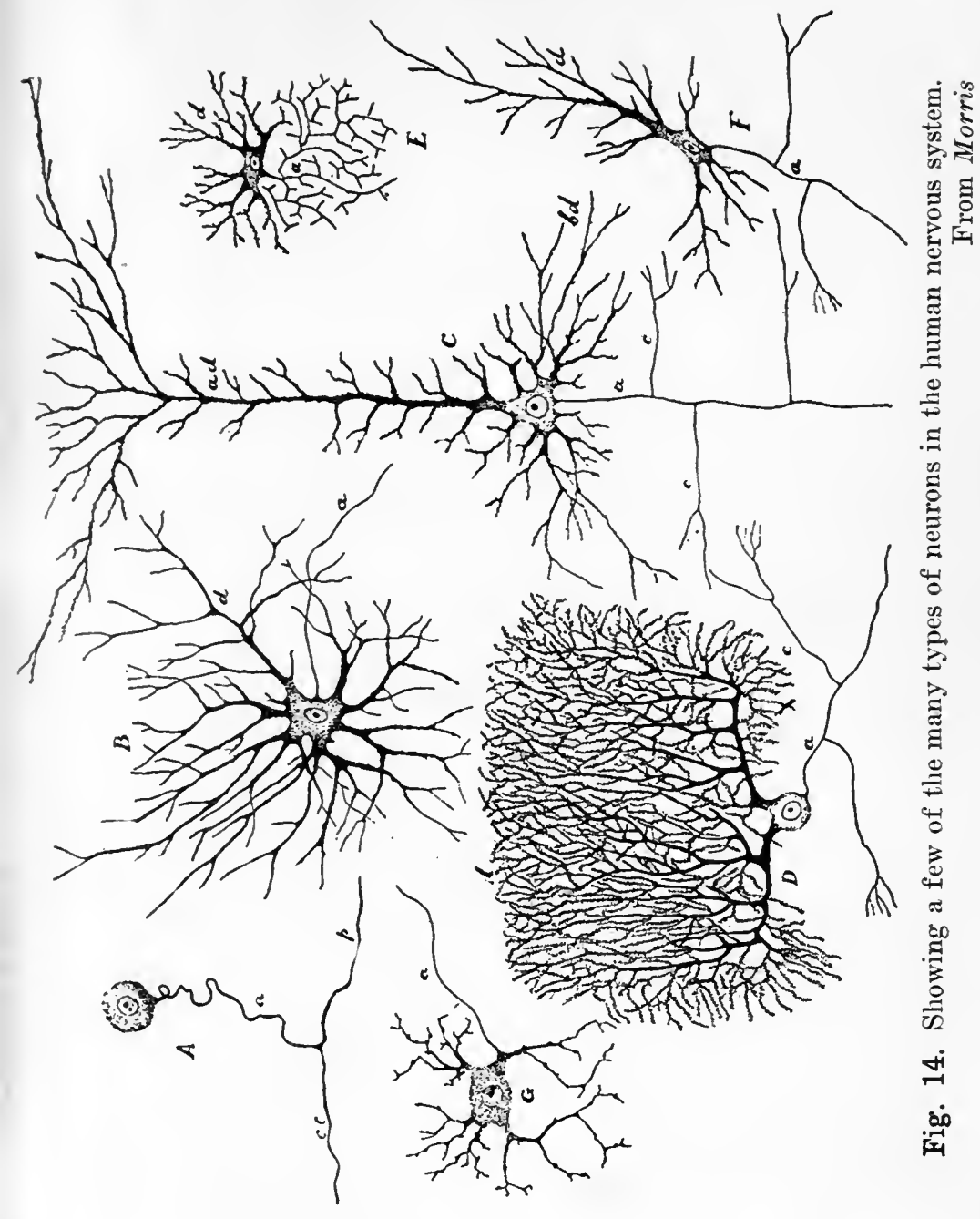




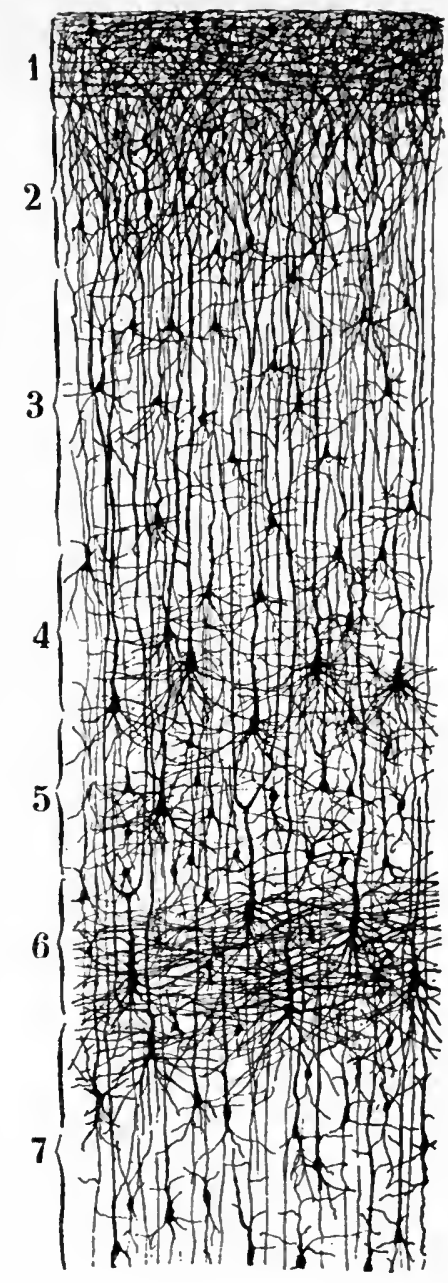

Fig. 15. Section thru a convolution of the cortex of a one month old child, showing structure and arrangement of neurons. Numbers at side slow the layers into which the cortex has been divided. 


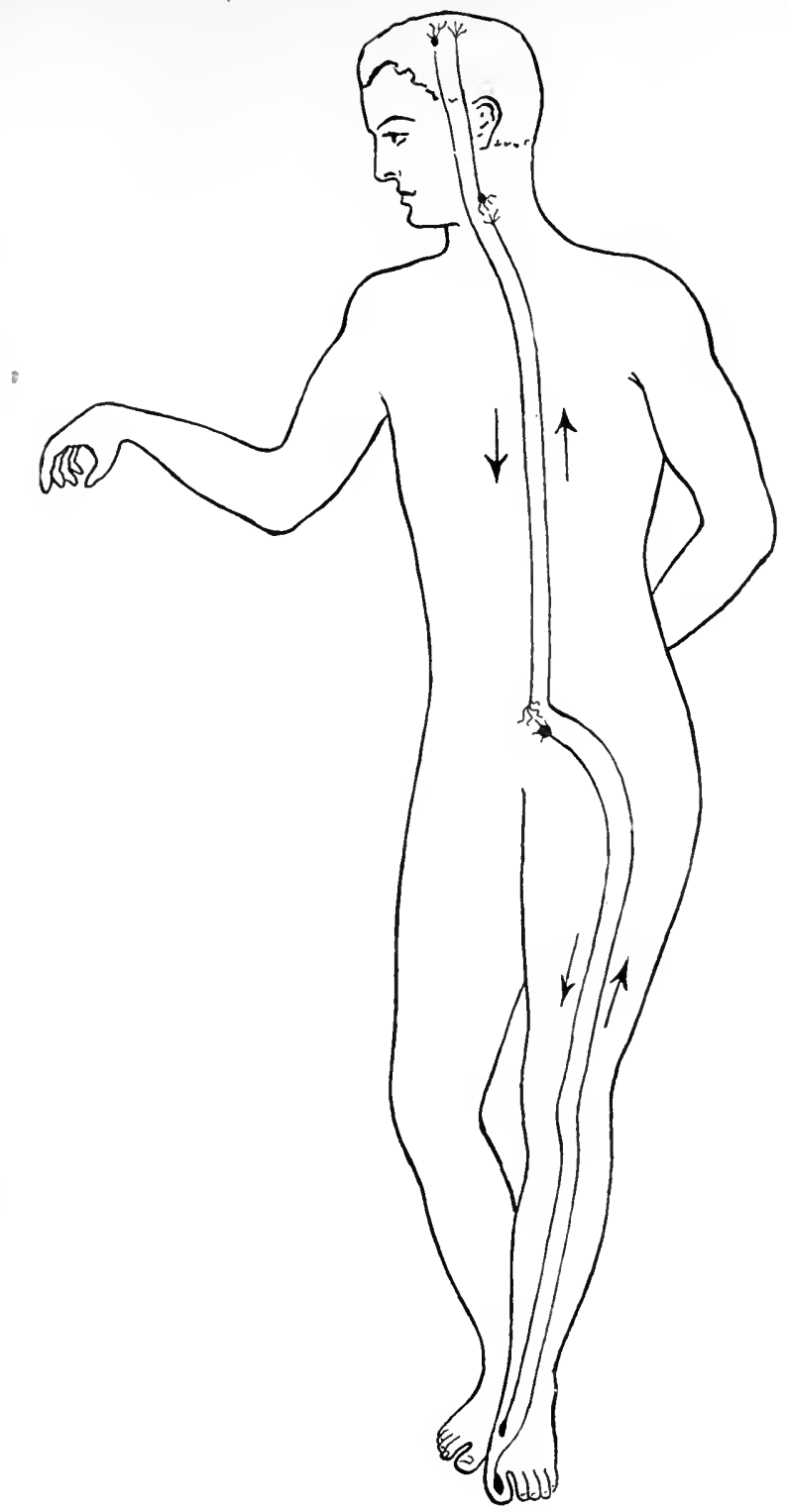

Fig. 16. Diagram showing the simplest neuron pattern from a stimulus applied to the toe producing sensation in cortex and returning to the muscle that moves the toe. Note the possible length of the various neurons. All these neurons would have many other possible connections not shown here. 


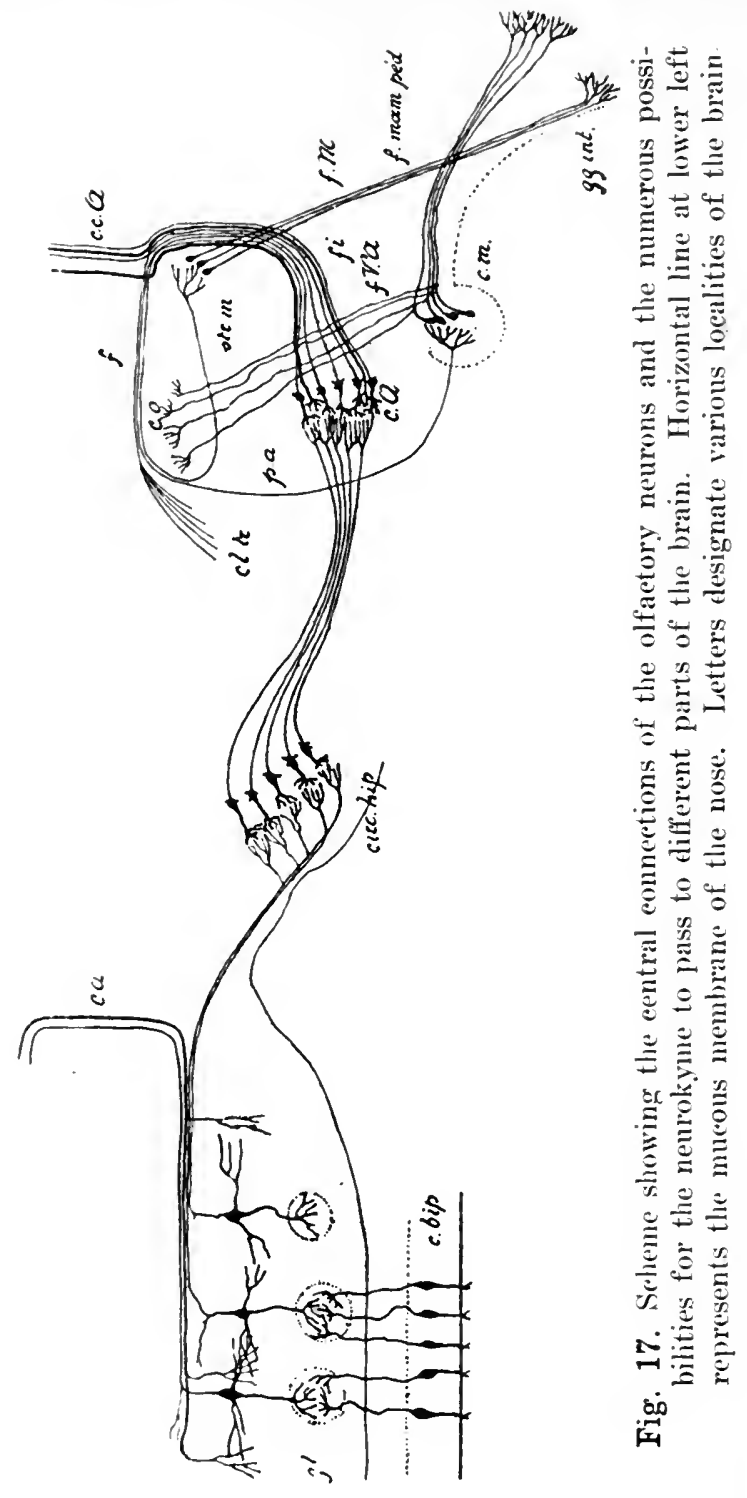



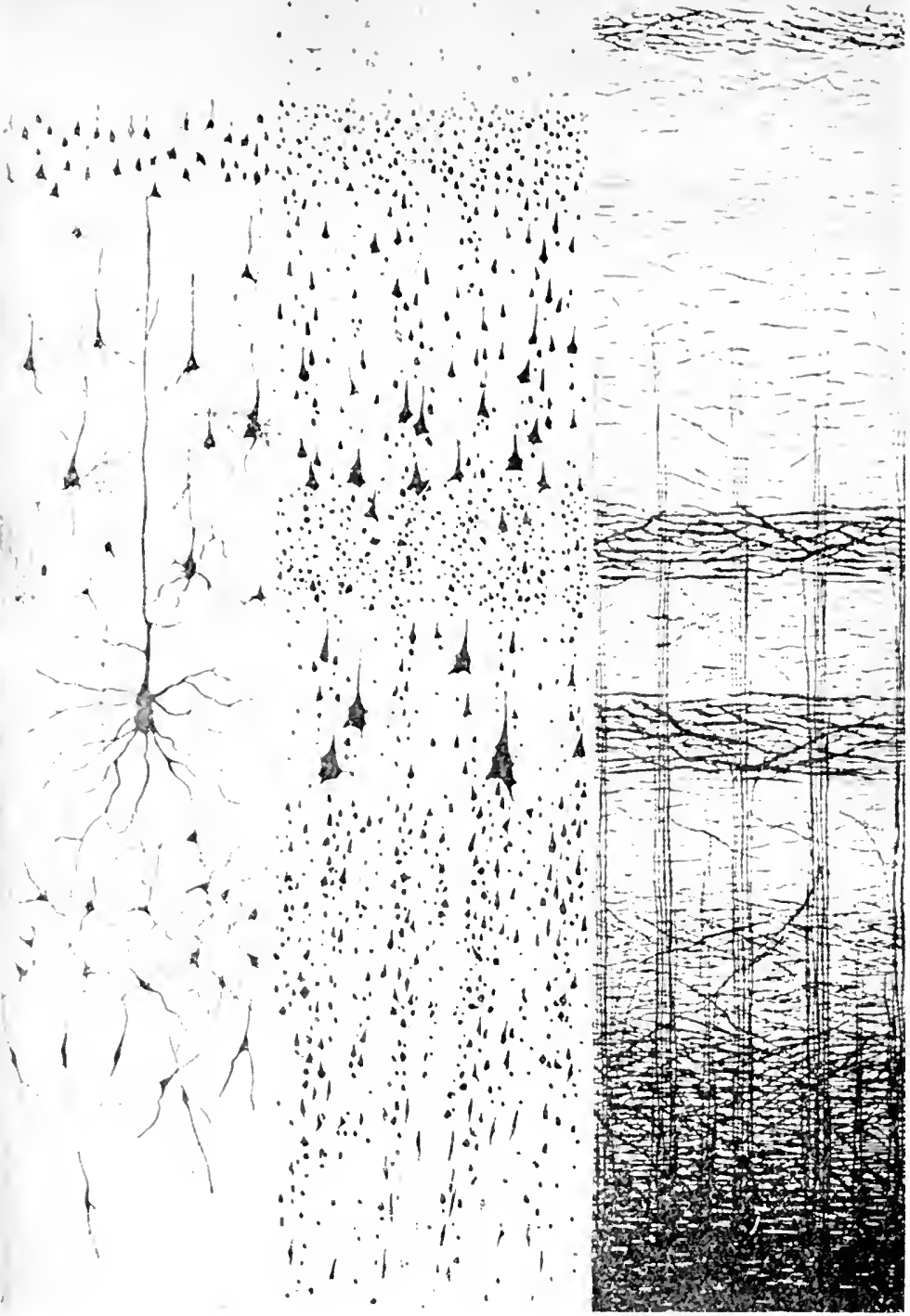

Fig. 18. Diagram to show the layers of cells and fibres in the grey matter of the human cerebral cortex, according to three histological methods: (a) Golgi; (b) Nissl; (c) Weigert.

From Luciani 



\section{NEURITES AND DENDRITES}

first spoken of. Here it also ends in several branches, one of which is in contact with a dendrite which is very short and whose cell body lies close at hand but whose neurite passes out thru the spinal cord and all the way down to the muscle of the great toe. Thus the stimulus from the pressure on the toe has travelled over this entire system and finally produces the movement of the toe.

It should be carefully noted that, whereas we have shown that at each point where one neuron comes in connection with another the neurokyme has taken a particular path, yet at each such point there are many possibilities. and innumerable other paths might have been followed. For example, the stimulus that we described as leading to a movement of the toe might by following other paths have led to an exclamation, or to a movement of the hand to remove the stimulus, or to any one of almost innumerable responses. Fig. 17 will give the reader a further appreciation of the complexity of neuron arrangement and the possibilities for different paths.

The reader must understand that in making these pictures of parts of an actual brain it is necessary to stain the different structures and that no one dye stains all the parts alike. Consequently it is necessary to use one stain for the dendrites and another for the cell bodies and different stains bring out different cell bodies in different ways. In Fig. 18 we have three sections from the same region prepared in three different ways. The right hand third shows the fibres, the middle and left hand parts show different kinds of cell bodies. These should really all be superposed except that the section would thus become so complicated that it would be impossible to see anything at all.

We must now return and consider our statement that 
the ten thousand million neurons are divided into groups each having its own rate of growth and its own function, or work to do. Let us consider the latter part first--the work to be done by each group. As is well known, we use the brain to hear with, to see with, to have sensations of pressure, to move various parts of the body and, as we say, to think with. There is one group of neurons that has particularly to do with seeing. The cortical cells that have to do with seeing are located at the very back of the brain in the occipital lobes. Those that have to do with hearing are located in the temporal lobes, parts of the cerebrum lying at the sides directly under the temples; and so on with the various other functions that will be best understood by a study of the diagram, Fig. 19 .

This mapping, in the brain, of the different functions is called brain localization, that is, the different mental experiences are localized in definite areas or "centres" of the brain. It will be noted that there are large areas here that have no functions assigned. Of these we shall have to speak later for they are of very great importance. We may call attention at this point to the fact that all these different areas are connected by what are called association neurons, that is, they connect or associate one part of the brain with another. Some idea of this will be obtained from Fig. 20; which shows the fibres (but not the cell bodies) connecting one part of the brain with another by various pathways. Besides this localization of function in different surface areas of the brain, it has been found that structurally it is possible to divide the cortex into lavers and recently it has been suggested that these different layers have different functions. The following quotation from Herrick (23, p. 289) will show the theory. 


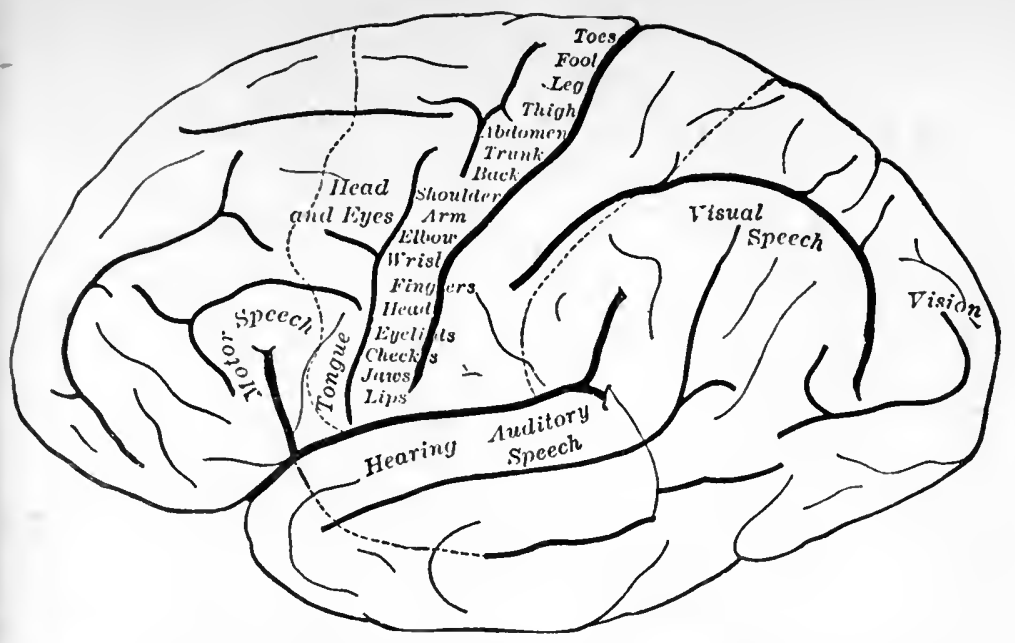

Fig. 19. The human eerebral hemisphere seen from the left side, upon which the functional areas of the cortex are indieated. This is brain localization. Note the large areas where no function is marked. These are the asscciation areas.

From Herrick after Starr

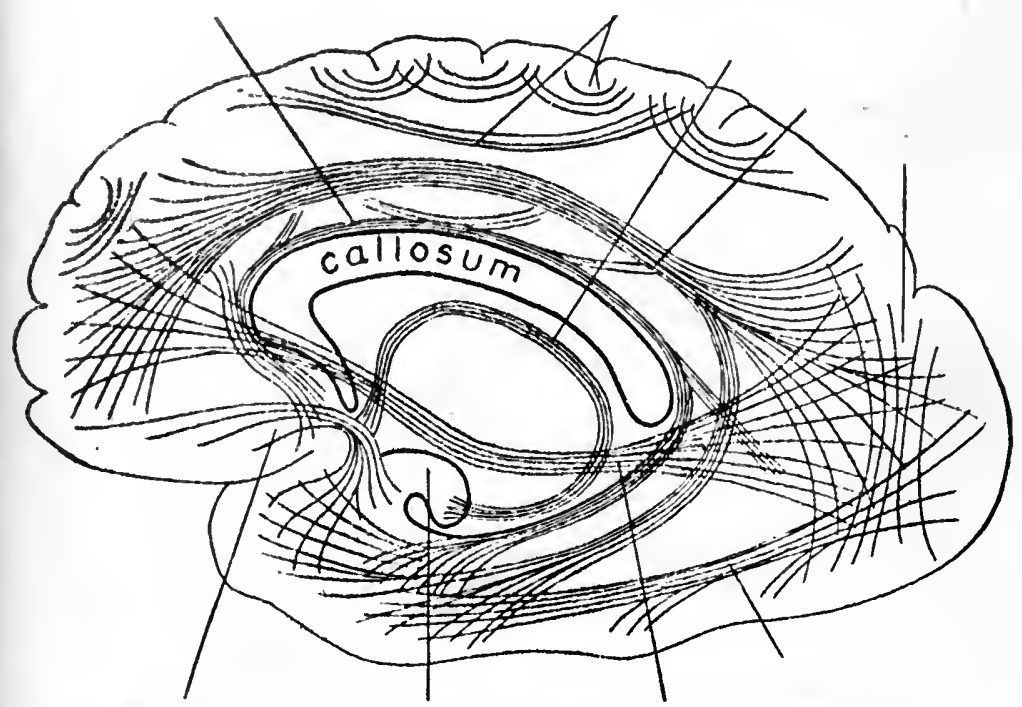

Fig. 20. Diagram illustrating some of the ehief association tracts of the cerebral hemisphere. Note how every part is connected with every other part of the brain.

From Herrick 



\section{NEURON LAYERS IN CORTEX}

"Several English neurologists, notably Bolton, from studies on the development and adult structure of the cortex in normal and abnormal men and in other mammals, have been led to the conclusion that, in addition to the mosaic localization pattern of which we have been speaking, there is a functional difference between the different layers of neurons of the cortex in general. Bolton believes that the granular layer marks an important boundary between functionally different cortical mechanisms. The infragranular portion of the cortex is thot to be concerned especially with the performance of the simpler sensori-motor reactions, particularly those of the instinctive type, while the supragranular layers serve the higher associations manifested by the capacity to learn by individual experience and to develop the intellectual life.

"The infragranular layers mature earlier in the development of the brain, and they are the last to suffer degeneration in the destruction of cortical cells in the acute dementias or insanities. The supragranular layers (notably the pyramidal neurons of Brodmann's third layer) mature later than any other layers. They are thinner in lower animals and in feeble-minded and imbecile men than in the normal man, and they are the first to show degenerative changes in dementia.

"This doctrine is controverted by some other neurologists, but the evidence seems to show that the supragranular pyramidal neurons are physiologically the most important elements in the higher associative processes of the cortex. In this connection it is significant that the granular and infragranular layers are thicker in the projection centres, while in the association centres the supragranular layers of pyramidal cells are thicker. But all of the layers in each region are very intimately related, 
the processes of most of the cells of the deeper layers extending thruout the thickness of the more superficial layers to reach the most superficial layer, and in the present state of our knowledge a functional difference between the layers cannot be said to have been established, save in very general terms."

We must now return to our other statement that these different groups of neurons grow at different rates and mature at different times. It is obvious that the particular mental process that is to result from the functioning of any particular group of cells cannot manifest itself until that group of neurons is developed. For example, a person could not see until the neurons in the sight centre had reached their complete development. Many animals are born blind. Their eyes do not open for several days after they are born. This is because the eye mechanism, probably including the cortical cells of the brain, is not ready for the stimulus of light. When it is ready the eyelids open and the animal sees. We shall find this principle playing a very important rôle in our later consideration of the mental processes. Fig. 21, A and B, will be found instructive in this connection as showing what one investigator has considered the order in which the various centres of the brain come to maturity and to complete functioning. This growth to complete maturity may be by one of several means, perhaps by an extension of the neurite and dendrite, or by the growth of the little branches at the ends so that the neuron can come in contact with other neurons.

By reference to Fig. $13 \mathrm{~A}$, it will be seen that if the right hand neuron is the normal, fully developed form, a neuron like any one of those to the left of this would not 


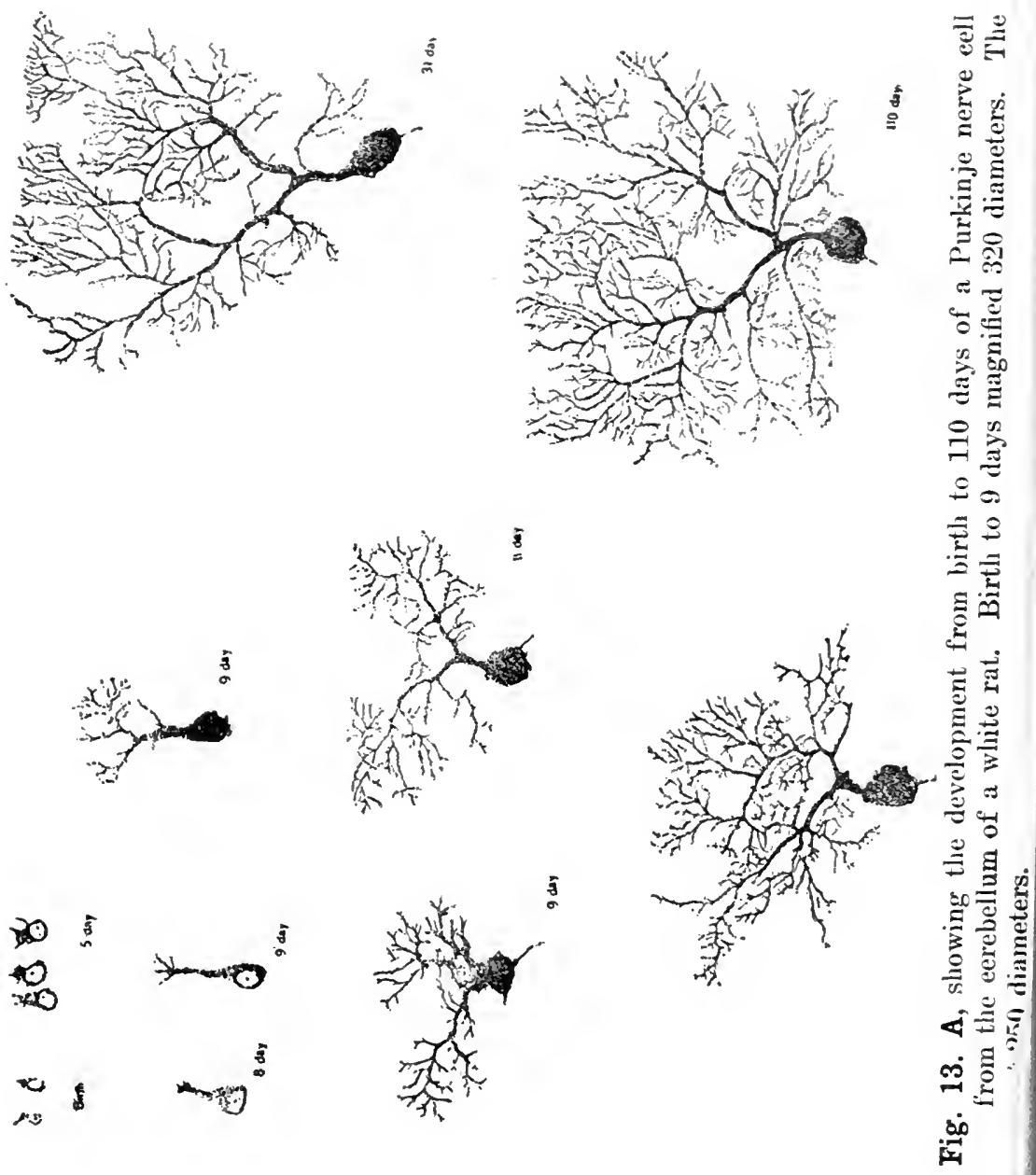


have as complete connection with the other neurons in the system and so would not be as able to receive or to transmit the neurokyme necessary to do its work. A person, therefore, with any group of neurons in the condition of these earlier ones, would be more or less deficient in whatever function was carried on by that group.

Besides being incomplete in size, neurons may also be incomplete in medullation. Each neurite and dendrite is surrounded by a covering known as its medullary sheath or medullation. Many neurons do not get this sheath until sometime after they have otherwise attained their maturity and it is believed that in the cerebrospinal nervous system the neuron does not function, that is do its work, until it gets that sheath. Consequently, neurons that have not yet developed the medullary sheath could not do their work. The function that was to be performed by that group would therefore be lacking. Flechsig (16) has shown (Fig. $22 \mathrm{~A}$ and B) that the child at birth has comparatively few medullated neurons in the whole brain but the medullation of certain fundamental tracts takes place very rapidly so that in a few hours, days or weeks there is a large amount of medullation. As we watch the child and see him develop and note his ever increasing mental capacity, we may easily imagine * that new groups of neurons are becoming medullated.

The neurons have been divided into afferent and efferent, sometimes called sensory and motor. More recently they are spoken of as receptors and effectors. Whatever terminology is adopted we understand that there is one group, the sensory neurons, or receptors that receive impressions (stimuli); there is another group that stimulate the muscles to action; these are the motor neurons or the effectors, and the system is so organized that 
we find it convenient to conceive that the goal of every stimulus is a muscle, that is, the circuit is complete when the energy that is started inward by the stimulus passes outward to a muscle. We shall see later that this goal is sometimes reached very quickly by a direct pathway, at other times by a very circuitous, round-about way. We shall also see that the more intelligent the being the more of this latter form of action we find.

Specific Stimuli.-While there are a great many neurons ready to receive impressions or stimuli and there are many kinds of stimuli to be applied, yet each different group of neurons responds only to its particular group of stimuli. The different receptors are modified for the purpose of reacting each to its specific stimulus. For instance, the neurons that are in connection with the visual centre of the cortex are modified to receive the stimulation of light. This special modification gives rise to what we know as the retina of the eye. Similarly we have a special modification for receiving the stimuli of wave motions in air which make sound. The ear does not respond to light, nor the eye to sound. Likewise we have special modifications in the skin for the sense of touch, in the nose for odours, in the tongue for taste. The structure and anatomy of these different end organs will be found in the proper books.

Not only does each group of neurons have its specific stimulus, but it is dependent upon such stimulation for its development. When the end organs such as eye or ear have been destroyed in early life by disease, it is found that the corresponding area of the cortex does not develop. Donaldson found from a study of the brain of the blind deaf mute Laura Bridgman that the cortex was 


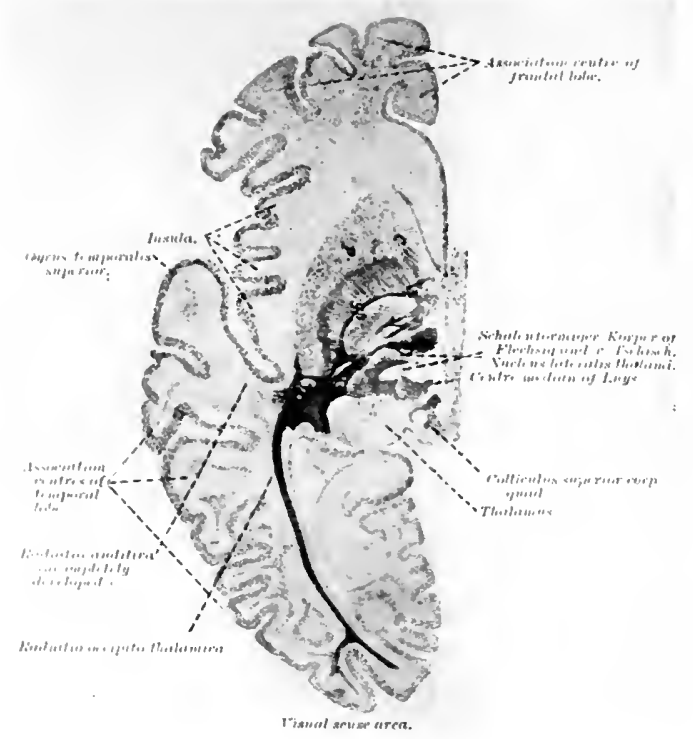

Fig. 22. A, section thru brain of child in the second week of life, showing medullated fibres (deep black) extending from visual area to contrai portion.

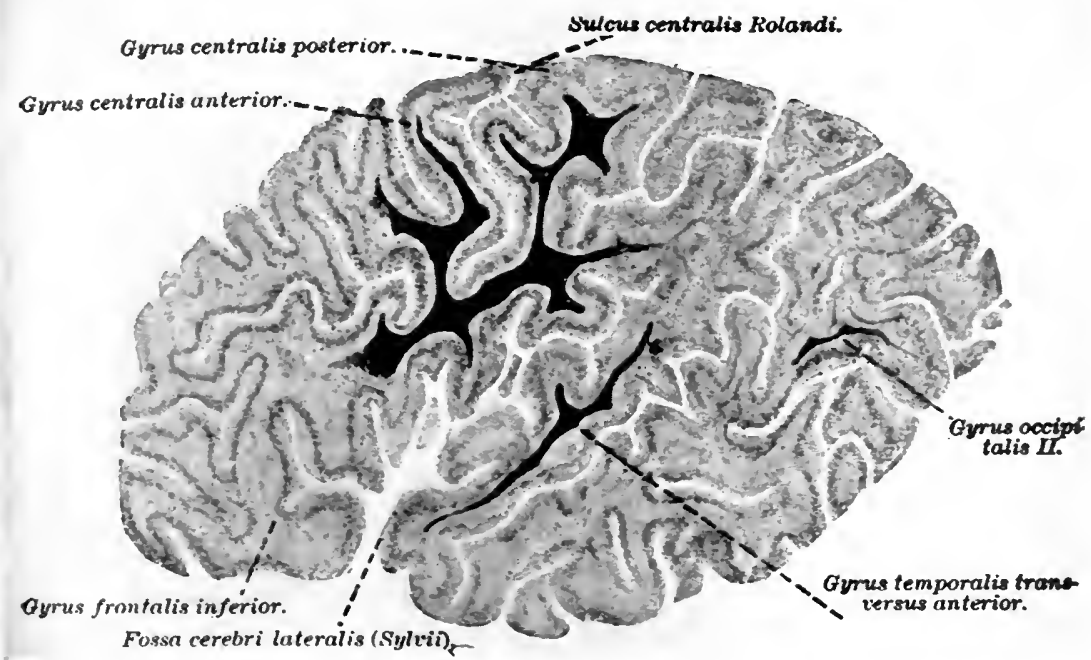

Fig. 22. B, similar section of brain of child at end of second month of life, showing medullated tracts in auditory area and in the sensori-motor region. In both $\mathrm{A}$ and $\mathrm{B}$ note the large areas as yet unmedullated. 



\section{LAURA BRIDGMAN'S BRAIN}

noticeably thin in those areas corresponding to the senses which had been lost. Laura Bridgman was a normal child who, as a result of a severe attack of scarlet fever at the age of two years became deaf and blind; and taste and smell were also impaired. She lived to be sixty years old. At autopsy the brain showed various abnormalities. Later it was carefully studied by Donaldson (13). The thickness of the cortex was carefully measured in fourteen different regions in each hemisphere and compared with the average normal thickness found by measuring homologous areas in nine normal brains.

Fig. 23 shows the result. The deviations from normal are greatest in the sight centres, in the hearing centres and centres for taste and smell. The nearest agreement with the normal is found in the "touch" centre, centre for head movements and other centres not so definitely located but which are probably motor centres. A very striking bit of evidence is seen in the difference between the sight centres of the right and left hemispheres. The cortex is much thinner in the sight centre of the right hemisphere than in that of the left. On referring to the history of her life it is found that while she was blind in the left eye at two years she had some sight in the right eye until she was eight years old. The optic nerves cross, hence this thin right hemisphere corresponds to blindness in left eye, and the better developed left hemisphere corresponds to partial sight in right eye until eight years of age. The evidence seems conclusive that the centres of the brain not stimulated did not develop.

Summary.-We have now laid out very schematically, 
and yet it is to be hoped with sufficient clearness, the plan of the nervous system. The facts that are important for our further understanding of mental processes are the following:

1. The brain grows to the age of approximately twenty years.

2. It not only grows in bulk but it grows in complexity.

3 . There are a great many different groups of neurons, each having its own function, its own rate of growth.

4. There is a very elaborate system of intercommunication between these neurons, so that every part of the brain is connected with every other part.

5. Because these neurons grow at different rates and come to maturity at different times, the different phases of the mind are developed at different rates and at different times.

6. There is an incoming and an outgoing movement of the nerve energy.

7. Each group of neurons has its specific stimulus and cannot be aroused to activity by any other stimulus.

We are now ready to understand what we mean by mind, first, in its simple processes and then, as it gradually develops, in its more complex processes. 


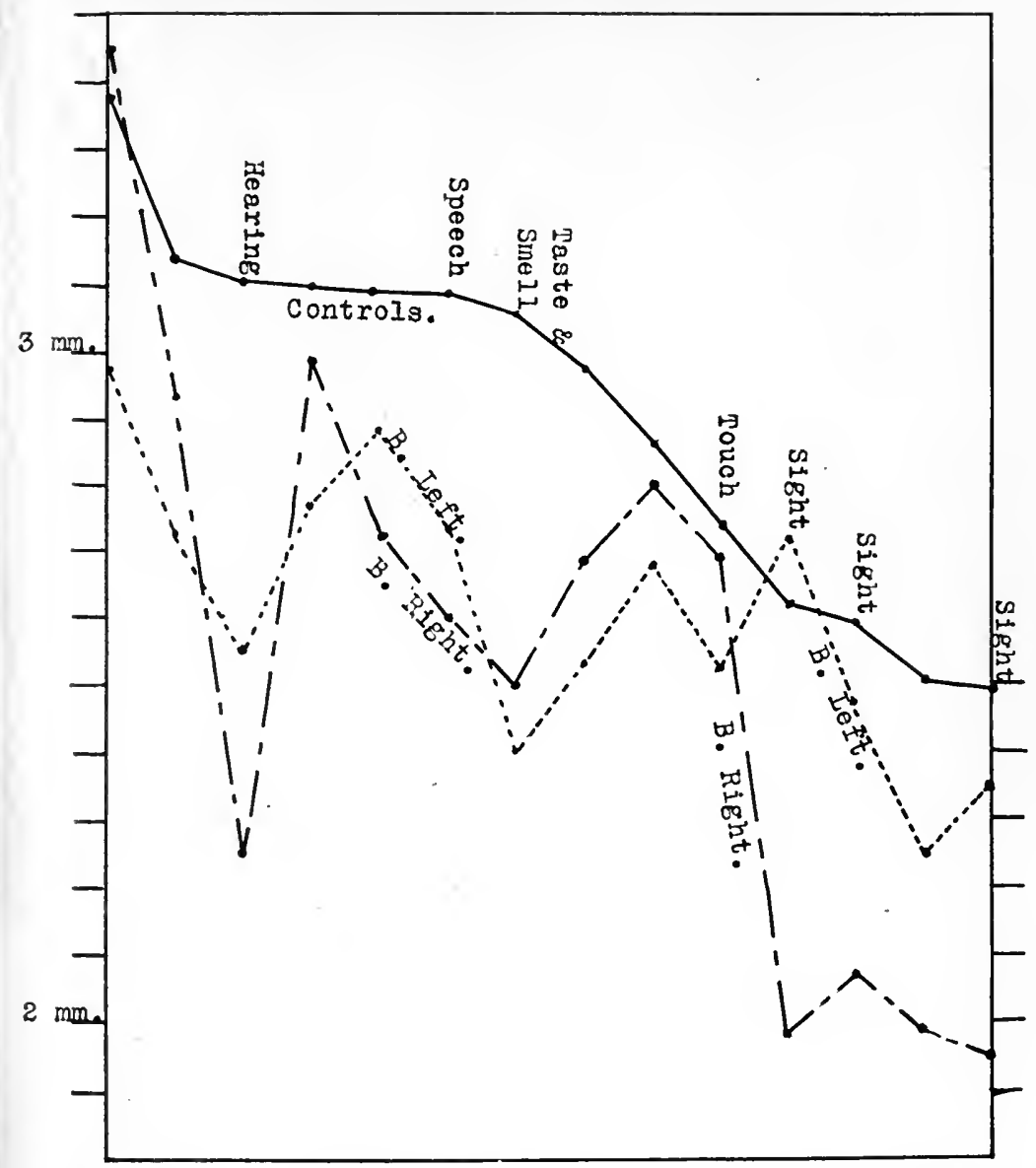

Fig. 23. Curves showing the thickness of the cortex in fourteen different areas of each hemisphere of the brain of Laura Bridgman in comparison with the average thickness of similar areas from ten normal brains. Figures at the side represent millimeters (and tenths) of thickness. Note that the cortex is thin in the areas corresponding to the lost senses; and nearly normal in the other areas. Compare also the sight areas of the two hemispheres. (See text.)

From Donaldson 



\section{CHAPTER II}

\section{THE BEGINNINGS OF MIND}

IN the previous chapter we have given a brief outline of the mechanism of the nervous system from which the student will understand something of the machinery which underlies mind.

Our next step is to try to see how the development of mind follows the growth of the nervous system. Something is known of the development of the nervous system and something is known of the development of mind. Our problem is to fit the two together to make as complete a picture as possible. We have two parts to our task: First, a consideration of the facts of nervous system and of mind; second, a consideration of such logical inferences as may be deduced from the facts. These deductions will enable us to form concepts from which further facts may be predicted. Certain facts of the nervous system enable us to formulate certain concepts in regard to mind and vice versa.

We will now consider the functions of the nervous system in the course of its development.

When a stimulus to the peripheral system, such as a pressure or a ray of light or a sound wave, is applied to any sensory dendrite, the nerve fibre is affected in such a way that some kind of transmission of energy takes place. This energy reaches the cell body and there liberates the stored up energy of the cell which passes on thru the neurite. It may then reach the nearby dendrite of some other 


\section{PSYCHOLOGY OF THE NORMAL AND SUBNORMAL}

neuron and the process be repeated. This energy, called neurokyme, is thus transmitted until it finally passes into a neurite which is in contact with a muscle and produces a contraction of that muscle. The nature of this energy for which we have adopted the suggested term neurokyme (nerve wave) is still a matter of dispute. According to some it is physical-in the nature of an electrical transmission; according to others it is chemical. It may be both. The term neurokyme must be understood as standing merely for a wave of energy passing thru the neuron-not that any substance passes from one neuron to another.

There are infinite possibilities of connection between all the different individuals of the ten thousand million nerve cells, - or such of them as are sufficiently developed to function. The simplest illustration of this is shown in Fig. 24, and may illustrate what takes place in a simple reflex such, for instance, as the well known patella reflex or knee jerk; the stimulus, applied in the form of a slight tap upon the nerve endings in the tendon, is transmitted to a cell body which is located just outside the spinal cord in a group of cell bodies known as a spinal ganglion. The neurite of this cell is within the spinal cord near or in contact with the dendrite of another cell which is thus stimulated. That cell body in turn is made to discharge its energy, which passes out over the neurite ending in a muscle which causes the foot to kick. This, as stated, is the simplest type of action known in the human body. From this simple circuit we progress to combinations so extensive, elaborate and complicated that no one can trace them.

It must be understood that while the afferent or sensory neuron is shown here in connection with only the 


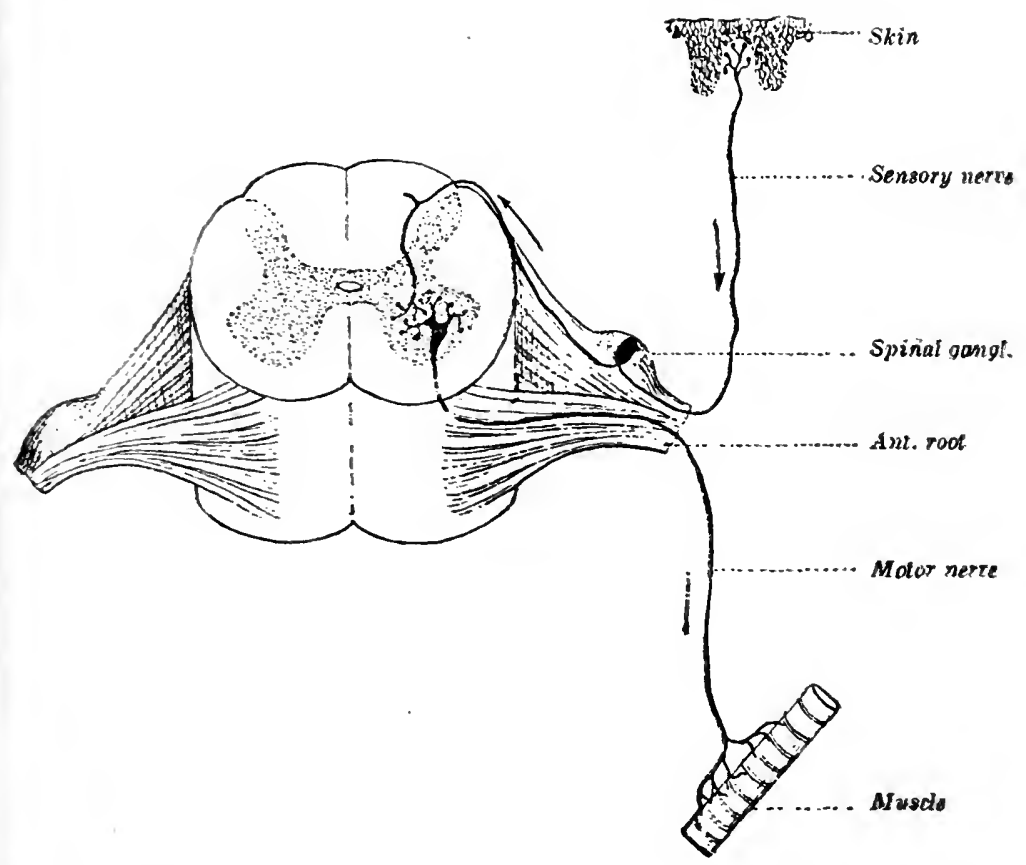

Fig. 24. Seetion of spinal cord showing simple reflex are from skin to musele. 



\section{THE NEURON PATTERN}

nearest motor neuron, in reality it has many branches and each branch may be in direct connection with another motor neuron, or there may be several or many intermediate neurons before the final motor neuron is reached. Thus the energy, started in the sensory neuron by the origial stimulus, has the possibility of travelling any one of a countless number of different paths. We shall hereafter speak of the group of neurons followed in any particular case as the neuron pattern.

The classic experiment with the pithed frog well illustrates not only some of the possibilities but the order followed. A frog is chloroformed and the spinal cord is cut behind the brain, thus removing all conscious or unconscious brain control. A drop of acid is now placed on the skin of the right side just in front of the hind leg. Immediately the right hind leg moves to wipe off the acid (remove the stimulus). Hold that leg and the other hind leg moves to the same end; hold this and the right fore leg takes up the action; hold this and the left fore leg moves in the same way. Apparently we can trace the course of the energy thru the different paths. When the right hind leg muscle contracts, the neuron pattern followed is the simple one pictured in Fig. 21. In the second case the neurokyme flows across the segment of the cord and out over the motor neuron to the muscle of the left hind leg. Thirdly, the neurokyme flows up the cord on the right side to the fore leg segment and out to the muscle of the right fore leg. Lastly, all these proving unavailable, it goes up the cord and across to the motor neuron leading to the muscle of the left fore leg. All these (and many other possibilities) are pure reflexes.

In man all these reflexes and many more occur. More[21] 
over, it is but a step to a most important phenomenon. Sometimes the neurokyme flows on up the cord thru the medulla to the cortex. Then consciousness may result. See Fig. $25 \mathrm{~A}$ and B. We shall consider that later.

The reader may ask why there is this multiplicity of neurons in a path instead of a single neuron extending from the stimulus directly to the muscle to be moved. The answer is two-fold: first, a saving of nerve material; and second, a saving of nerve energy.

For the first let us take the telephone as an illustration. If six homes are to be connected by single wires we should have something like Fig. $26 \mathrm{~A}$. But if a single relay station or switchboard is introduced we have the much simplified $26 \mathrm{~B}$.

For the saving of energy we may take our illustration from the telegraph. Almost every one understands that telegraphy is a system of clicks on an instrument which is actuated by electricity. The apparatus necessary is a galvanic battery, wires, a telegraph sounder and a key for making and breaking the circuit. If the key is open (circuit disconnected) the sounder is up. When the key is pressed down (closed) the circuit is closed, the current passing along the wire makes the magnet pull the sounder down causing the click. The key may be in one city and the sounder in another; the only thing necessary is to have the wires lengthened to reach between the cities. But, few people understand that in practice this is not exactly the situation. The reason for this is that electrical energy is lost as it passes over miles of wire and the cells of battery which would operate a telegraph system from one room to another, or from one building to another one nearby, would not operate it if the key, sounder and battery were miles apart, for the reason 


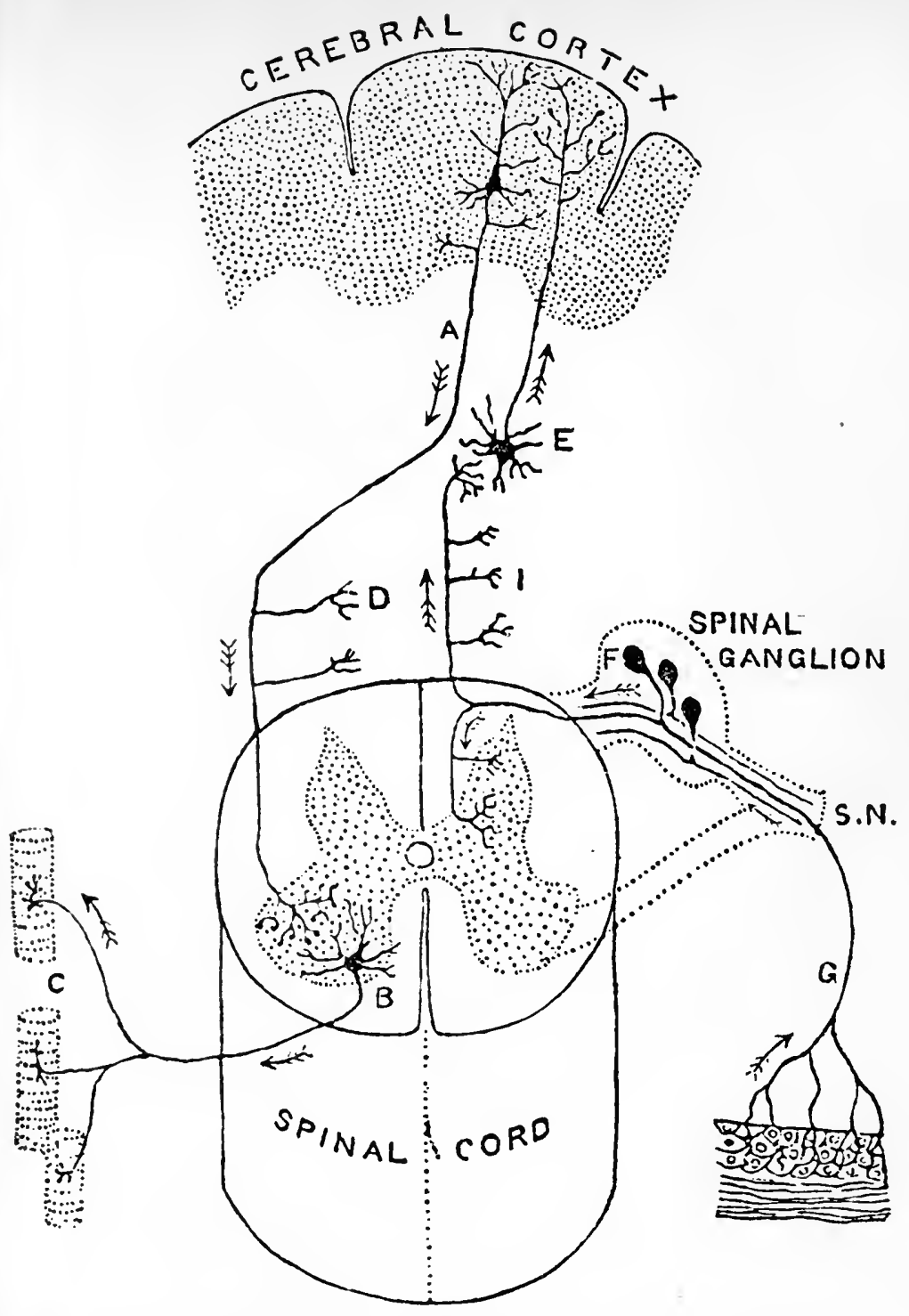

Fig. 25. A, diagram of the simplest possible reflex are from epidermis thru cerebral cortex to striped muscle. The are as drawn involves four neurons. According to some, there is another synapse between $\mathrm{E}$ and the cortex (see B). There may be intermediate neurons in the cortex, forming links between the afferent neuron $\mathrm{E}$ and the efferent neuron $\mathrm{A}$. 


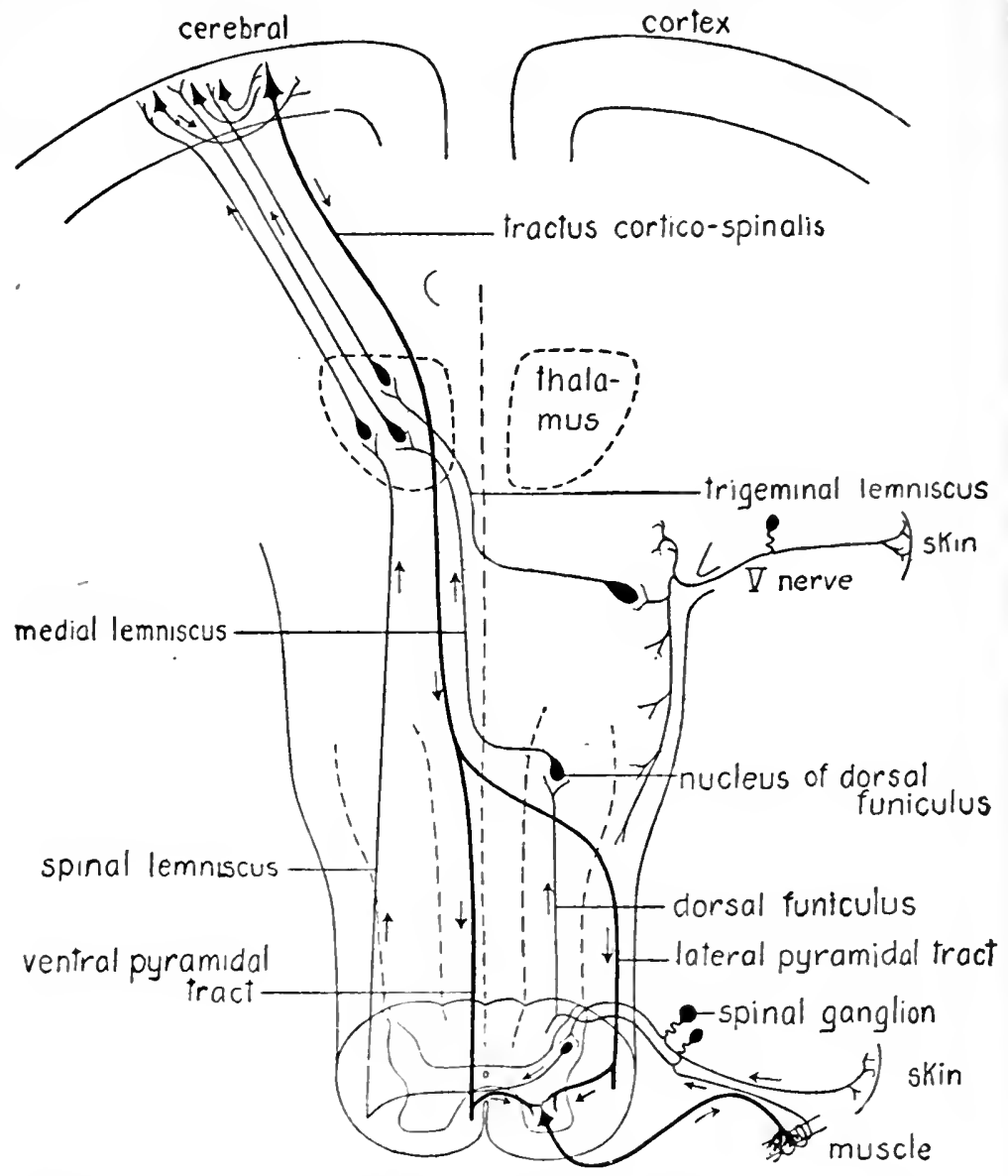

Fig. 25. B, similar to $A$ but showing the extra synapse in the thalamus and showing the pyramidal tract (motor, efferent)heavy line-part of which crosses over as do most tracts from left side of the braun to right side of eord; and another part which does not cross. The latter is known as the direct pyramidal tract. 
. 


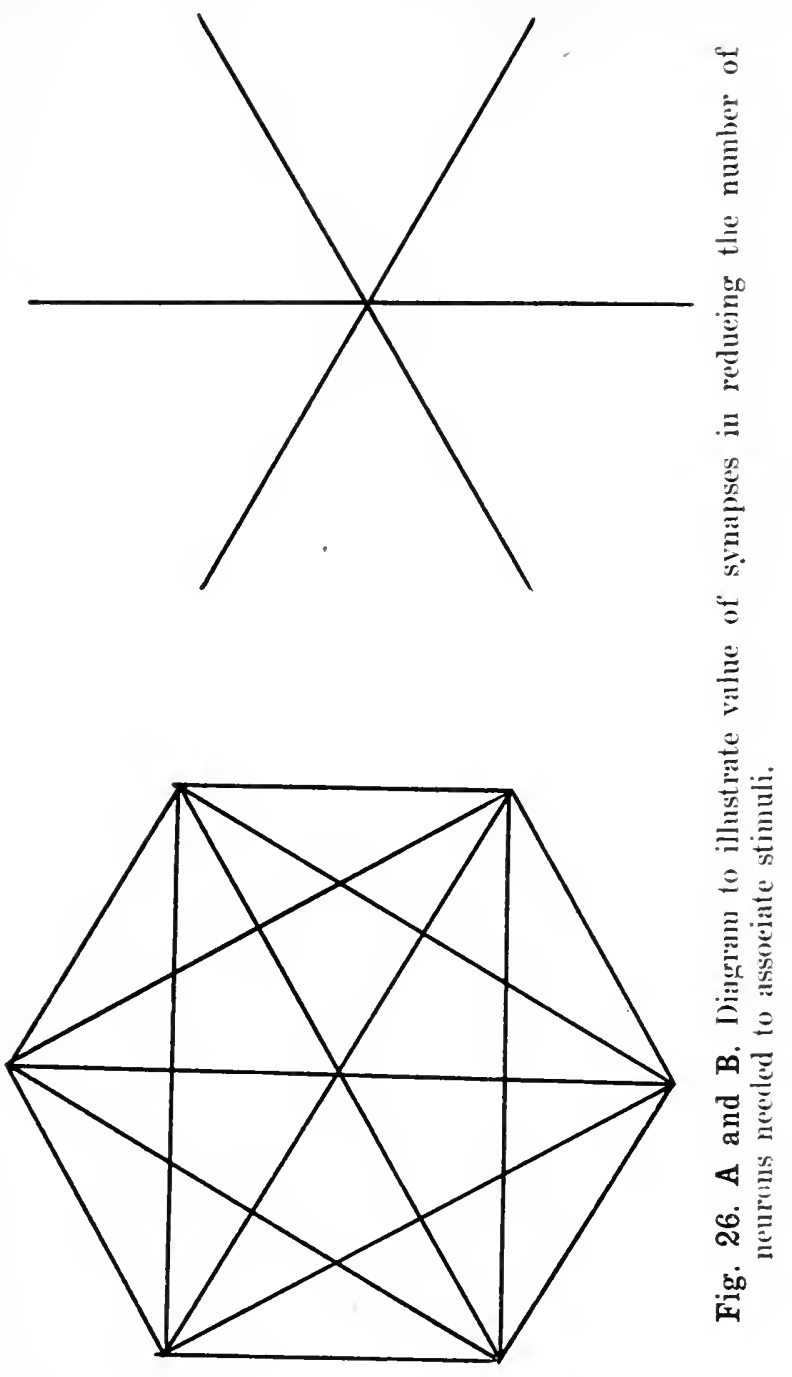




\section{NEURON PATTERNS}

that the battery would not be strong enough. Increasing the size of the battery offers only a partial solution. The difficulty is obviated by the use of what is known as a relay. The relay is an electro-magnet with an armature so delicately balanced that it requires only an exceedingly small current to move it. The electric current, which would not move the telegraph sounder sufficiently to make any sound whatever, will actuate the relay. The relay throws into the circuit a local battery which will actuate the local sounder. If it is a question of telegraphing a long distance, the relay throws into circuit another length of wire and another battery which will work for a certain number of miles, throw in another relay, and so on across the country, sounders being placed wherever they are wanted.

This will be easily understood from the accompanying diagram, Fig. 27 , and it is only necessary to change the name to make this illustrate what may be assumed to be the operation of nerve energy in the nervous system. Call each battery a cell body, each bit of wire a nerve fibre and each relay a synapse or point where one nerve fibre either touches or comes so close to another nerve fibre that the energy passes across and so sets the new cell body into activity. In some such way apparently nerve energy is transmitted thruout the nervous system.

It must be remembered that all that we actually know about this in the nervous system is the fact that there are these elements, the neurons with their cell bodies and projecting fibres, and the fact that energy is transmitted and that movements result from the stimuli.

Neuron Patterns.-On page 12 we set forth the generally accepted facts of brain localization, that is, that one part of the cortex gives rise to consciousness of sight, 
another to hearing, another to taste, another to smell, another to skin sensations, etc. In the immediately preceding pages we have developed the idea that the simplest possible neuron action, that of two neurons, sensory and motor, gives rise to a simple reflex act. It has also been stated that this separate act may be complicated by the inclusion of more neurons. We have shown a simple cortical path in which there were four neurons involved (Fig. 25).

All the foregoing as well as the evolution of the nervous system as we go up the animal series warrant us in concluding that the fewer the neurons involved, the simpler the action; and the more neurons, the more complex the action; and we may safely assume that when we are considering consciousness, the same relation holds-a fairly simple neuron pattern will underlie a fairly simple state of consciousness, a more elaborate state of consciousness will be dependent upon a more elaborate neuron pattern. To make this clear we picture the accompanying hypothetical neuron patterns; Figs. 28 to 31 , some simple, some more complex and some rather highly complex.

The reader must understand that, while these pictures are actual drawings of sections of the cortex, our use of them to illustrate neuron patterns is purely hypothetical and made simply to enable us to have some kind of mental picture of what may be the brain condition for various states of thot and mental processes

Consciousness.-Mind is almost universally considered as in some way synonymous with consciousness. Yet psychology has been compelled always to go somewhat beyond the limits of consciousness in attempting to explain mental phenomena. Long ago popular usage developed 


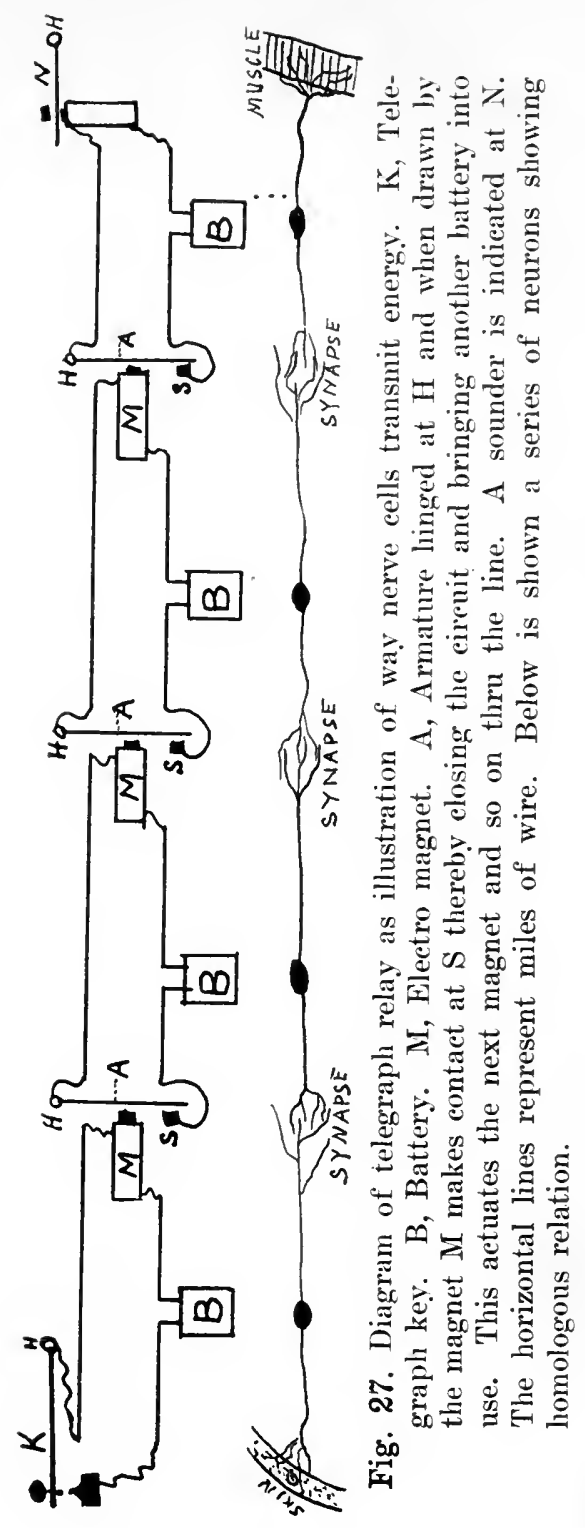




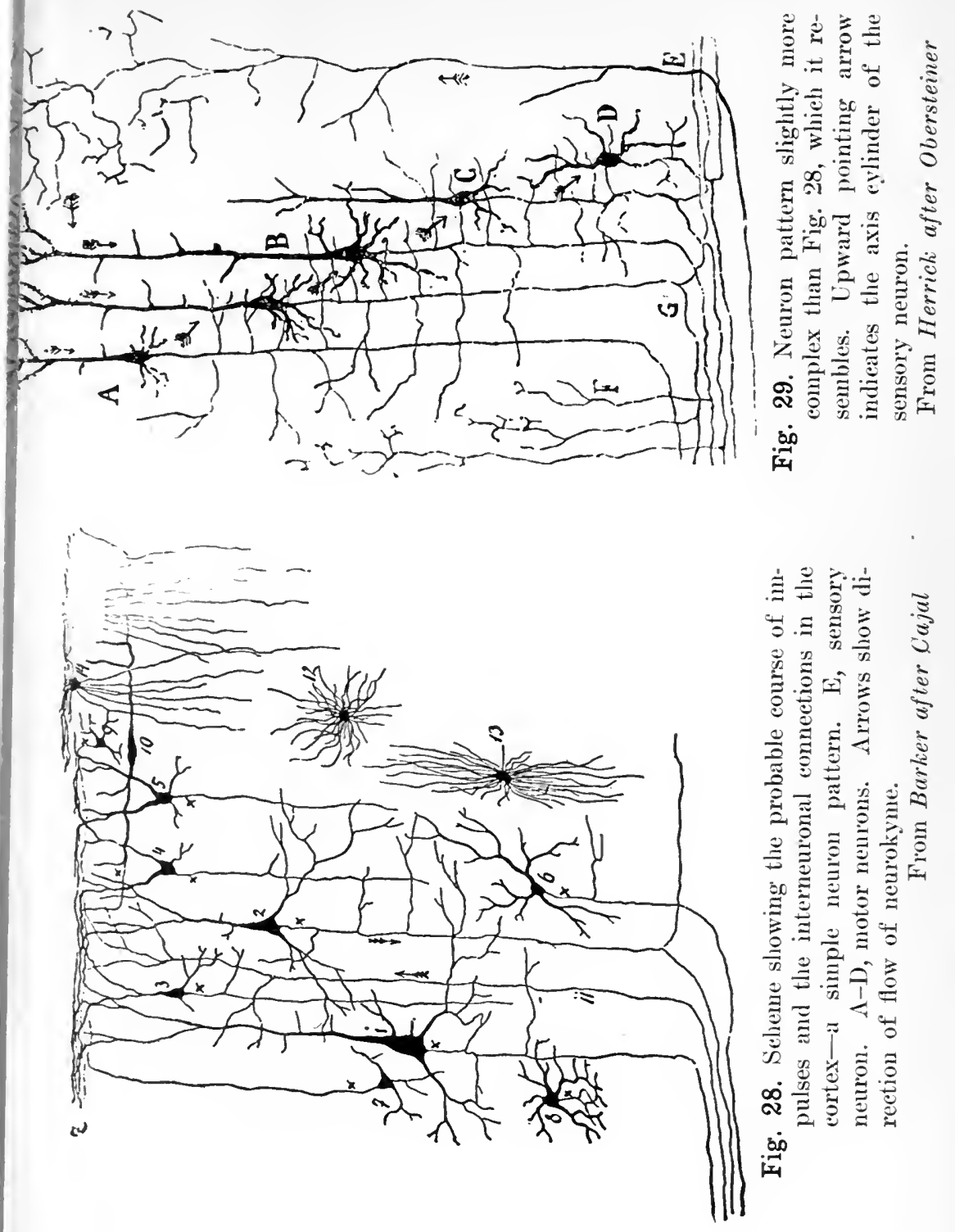


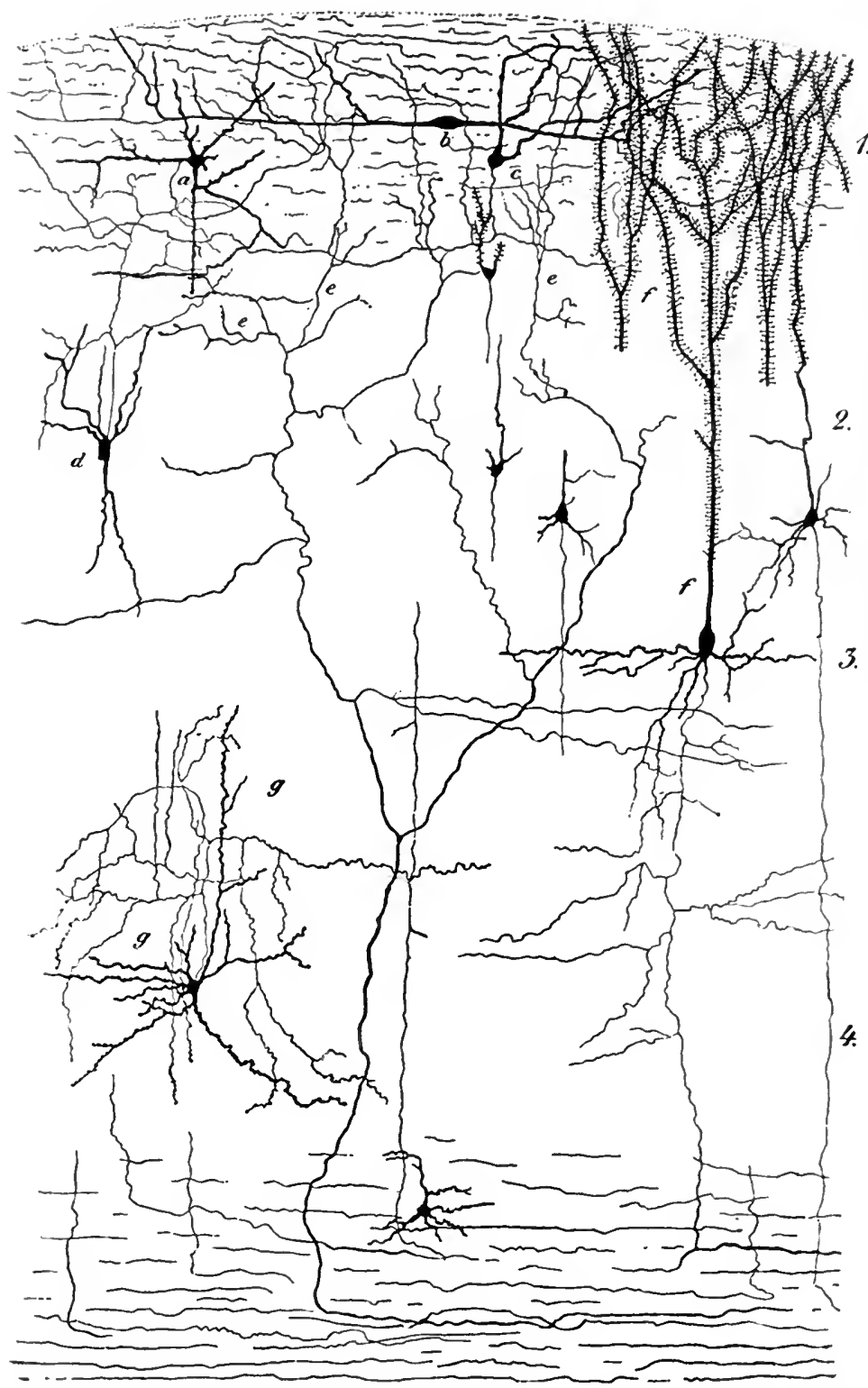

Fig. 30. Illustrating a relatively complex neuron pattern. The widely branching sensory neuron (marked e) is seen reaching to many different types of association and motor neurons.

From Edinger 


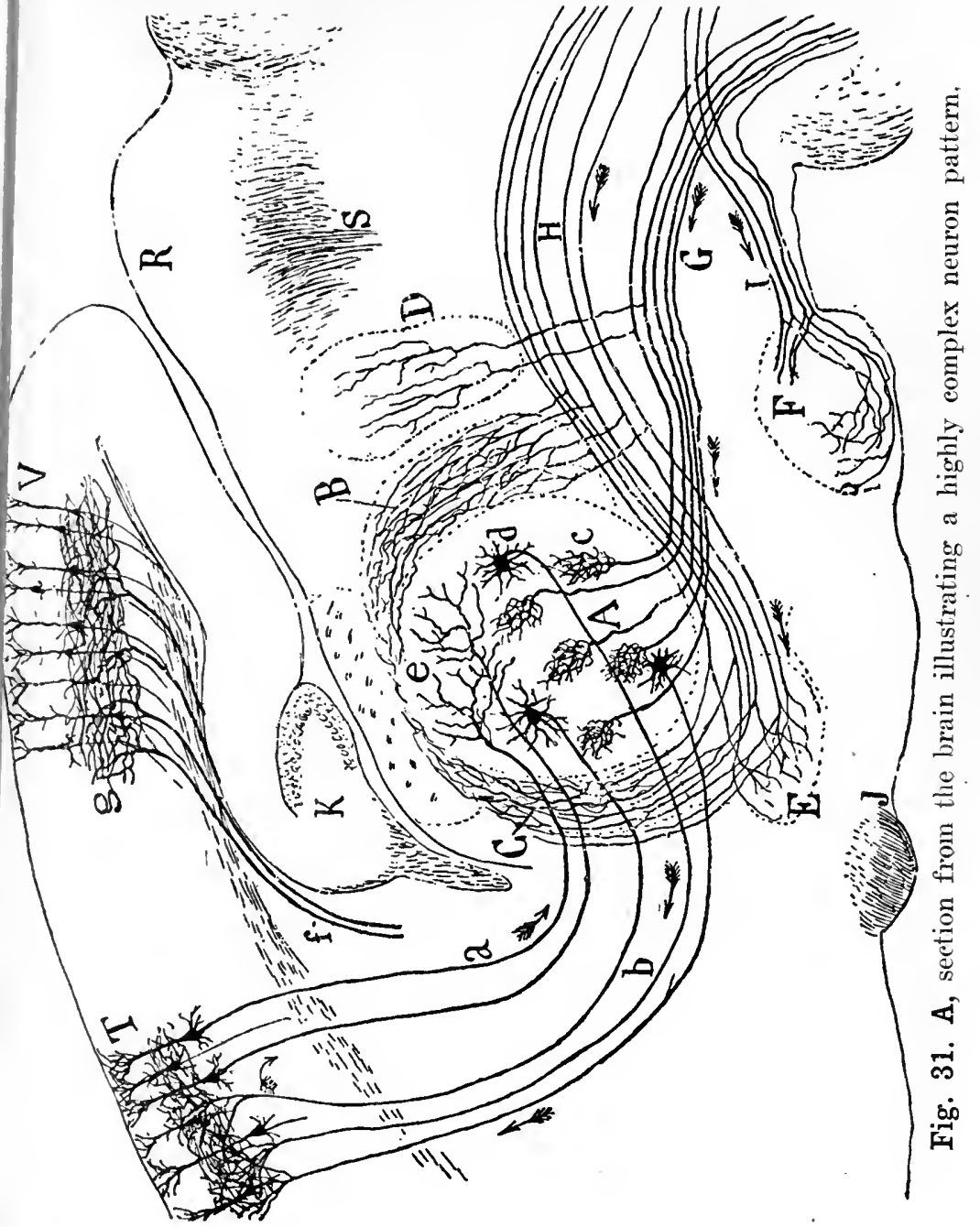




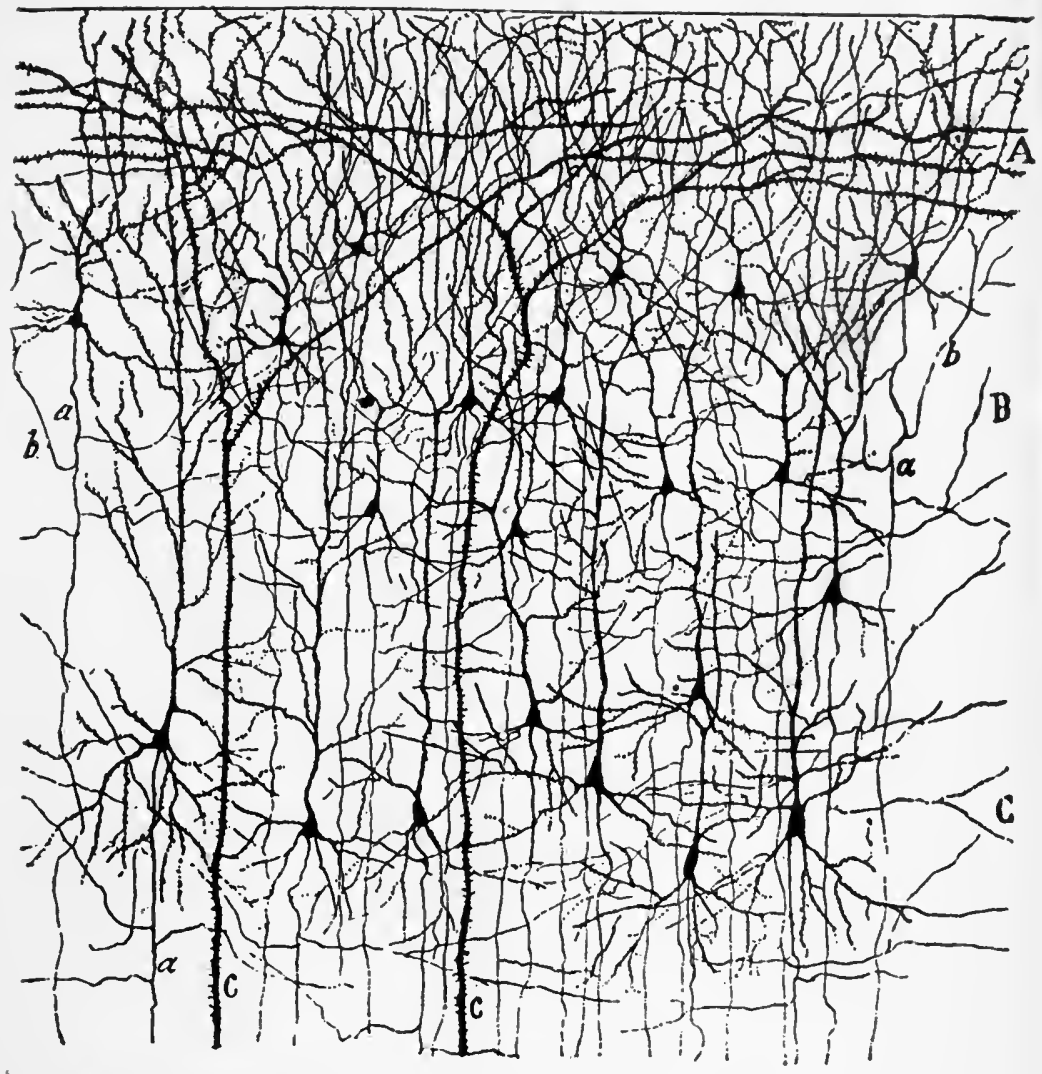

Fig. 31. B, detail of A. Section of cortex showing the multiplicity of possible patterns.

From Cajal 
the term unconscious mind to explain certain phenomena that were not conscious and yet to the layman seemed to be so much a part of mind as to deserve to be designated in this way. The term unconscious mind is of course a ridiculous contradiction. More recently scientists have gotten around this absurdity of terminology by speaking of "the unconscious" or "the sub-conscious." The study of the behaviour of animals and of man gets away from this difficulty by saying nothing about consciousness but putting all the emphasis upon the behaviour.

It seems clear that whatever be the difficulties of terminology they must be somehow surmounted, because psychology must deal with all the phenomena that result from nerve action, whether they be conscious or not. The figure of the iceberg has been used as a suggestive illustration of the true status of things; just as seveneighths of the iceberg is below the surface and out of sight and only one-eighth is visible, so there is a great submerged element which is part and parcel of what we call mind, and our preference for applying the term mind to the visible part only, must not blind us to the existence of the other part or to the importance of understanding it in order to know fully about the visible part.

Starting then with nerve action, we found the simplest mechanism in what is known as the reflex-arc. We have seen that Fig. 21 shows the simplest form of this nerve action. It represents (1) the organ for receiving the stimulus, for example, the skin, or tendon; (2) the sensory neuron whose cell body is in the spinal ganglion and whose neurite passes into the posterior part of the spinal cord and crosses over on the same level to the anterior side where it comes in contact with (3) a motor neuron thru which the neurokyme flows out directly to (4) the 


\section{PSYCHOLOGY OF THE NORMAL AND SUBNORMAL}

organ involved, the muscle. Here we have only two neurons, the stimulation of the sensory or afferent neuron leads directly to the movement of the muscle thru the motor or efferent neuron.

From this simple beginning we shall find the mechanism for most elaborate extensions increasing the number of neurons indefinitely. This first action, as pictured in Fig. 21, is a pure reflex and is believed to be absolutely without consciousness; but somewhere in the series, we do not at present know where, consciousness enters, and we have first a simple consciousness and then a more elaborate form and finally the most elaborate of which the mind of man is capable. The various steps must be traced out as carefully and elaborately as our knowledge will permit.

We must now consider briefly the advent of consciousness in relation to our problem. Reflexes take place whether the person is conscious or not. A child sound asleep and entirely unconscious will withdraw his foot if it be tickled. While awake we close the eyelids every few seconds to wash the eye ball and we are never conscious of doing so unless something definite calls it to our attention. It is a reflex and as such is normally unconscious.

Similarly a great many functions and activities of various organs of the body are controlled by reflexes and the normal man in good health has no consciousness in regard to them. On the other hand, a loud noise or a bright flash of light in the eye or pressure upon the skin produces consciousness.

We have already suggested that when cortical neurons are involved in the nerve path we get consciousness. Two cautions are here necessary. We are not sure that 


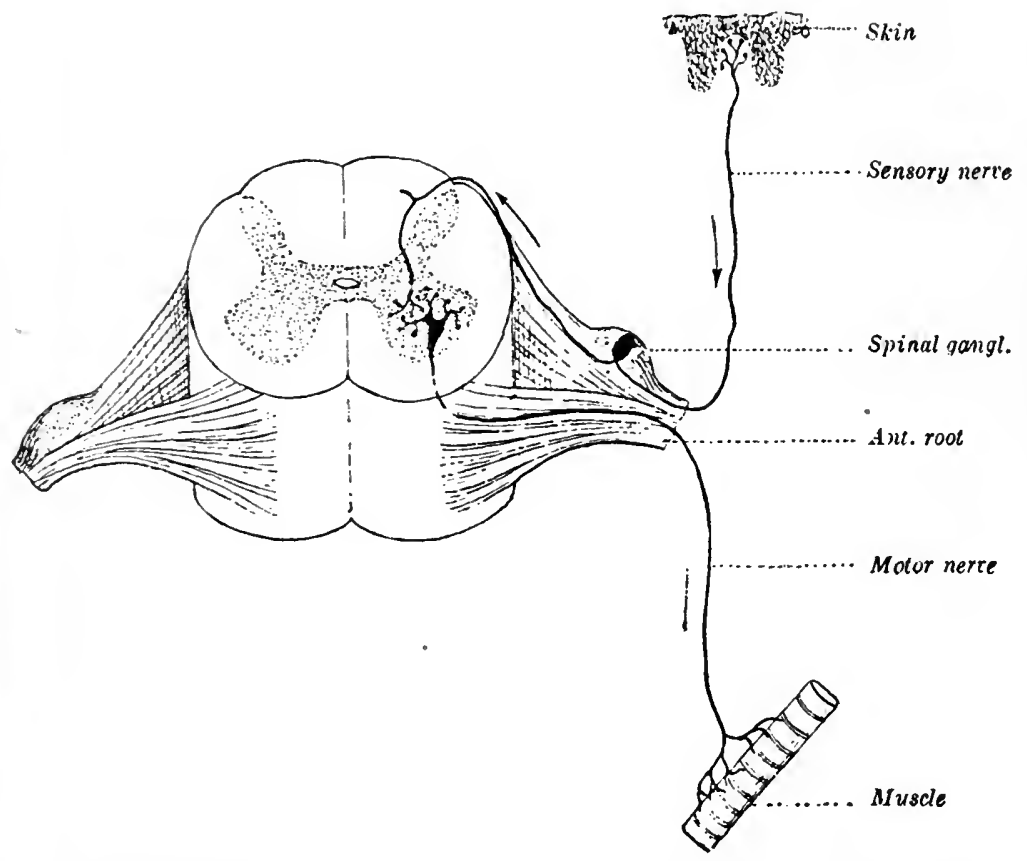

Fig. 24. Section of spinal cord showing simple reflex are from skin to muscle. 

all cortical action results in consciousness and secondly the statement does not necessarily mean that the cortex is the "seat of consciousness"; but both clinical experience and cortical structure make it fairly clear that at least there is no consciousness without cortical action.

Herrick (23, p. 291) says: "The higher mental proc" esses undoubtedly require the activity of association centres of the cerebral cortex, and the integrity of the associated mechanism as a whole is essential for their full efficiency. The cerebral cortex differs from the reflex centres of the brain stem chiefly in that all of its parts are interconnected by inconceivably complex systems of associational connections, many of which are probably acquired late in life under the influence of individual experience, and any combination of which may, under appropriate conditions of external excitation and internal physiological state, become involved in any cerebral process whatever."

Just how consciousness results from nerve actionwhether that action be in the cortex or elsewhere-is not known. So little is known in fact that there are not even accepted theories. Yet it may be helpful to consider some crude analogies. We have drawn upon the telephone and telegraph for illustrations of nerve action. The temptation is strong to see still other analogies. When two wires charged with a strong current are connected by a small wire or are allowed to barely touch each other the current, formerly imperceptible to any human sense, suddenly becomes manifest as heat. May it be that two nerve fibres (neurite and dendrite) in contact or in close juxtaposition make manifest the energy otherwise imperceptible? If so Herrick has furnished us with a ready understanding of why "consciousness is located in 
the cortex"-because of the "inconceivably complex systems of associational connections." In the cord and in the encephalon with the exception of the cortex the connections are few and simple. The "consciousness" resulting from two or even twenty neurons is too faint to be consciousness, but when thousands are involved it rises above the threshold.

The foregoing suggestions are in accord with existent hypotheses. For example, it has often been suggested by psychologists who deal with this phase of the problem that consciousness, especially in the higher thot processes, is in some unknown way the result of some interference with the free flow of the neurokyme. On the neurological side there is some evidence, not yet conclusive, that the nerve processes, the ends of the neurites and dendrites, have what is called ameboid movements, that is, they push out and also contract, the former movement of course bringing the various ends closer together, enabling the neurokyme to flow more easily, the latter movement drawing them apart and offering greater resistance. The following quotation from Herrick (23, p. 104) states the matter very concisely yet clearly:

"The numerous theories regarding the neurological processes taking place in the cerebral cortex during the progress of such mental functions as attention, association of ideas, etc., are likewise as yet entirely unproved. It has been suggested that during cerebral functions the resistance of some pathways may be diminished by the ameboid outgrowth of the dendrites so as to effect more intimate synaptic union with the physiologically related neurons, while the resistance of the other paths may be increased by the retraction of dendrites from their synapses. Others believe that the neuroglia may participate 


\section{AMEBOID MOVEMENTS IN NEURONS}

in the process by thrusting out ameboid processes between the nervous terminals in the synapses and thus increasing the resistance. Lugaro has suggested a different interpretation, in accordance with which during sleep there is a generally diffused extension of all nervous processes, thus providing for the uniform diffusion of incoming stimuli, while in the state of attention all of these processes retract save those which are directed in some definite direction, thus narrowing the stream of nervous discharge so as to intensify it and direct it into the appropriate centres. There is no direct evidence for any of these theories, and the scientifically correct attitude toward them is frankly to admit that at present we do not know what physiological processes are involved in any of these functions."

The view of Lugaro above referred to by Herrick would seem to account nicely for the difference between spinal cord neuron patterns and cerebral patterns. It also explains fairly well what has been a very difficult problem; why so many of our common activities, which are at first performed with very decided consciousness, later come to be performed with practically no consciousness. For example, the first time one winds a watch he attends to the winding to the exclusion of practically everything else, but he soon comes to the point where he winds it so unconsciously that he does not know afterwards whether he has wound it or not. We could imagine that on the first occasion he is establishing a new neuron pattern, and since it is new, the nerve endings have not grown out into contact with other nerve endings and consequently there is considerable difficulty in the passage of the nerve energy. But upon the second occasion these neurons are found in a little better contact, and upon third and successive occa- 


\section{PSYCHOLOGY OF THE NORMAL AND SUBNORMAL}

sions still better, until finally they are in such close contact that the stimulus has only to be given by the sight of the watch or the time of day when it is usually wound for the whole process to run smoothly from beginning to end without any impediment or interrupton and consequently without any noticeable consciousness.

Spinal cord and other inherited patterns would also be explained. Since they underlie actions long since perfected in the history of the race, those nerve endings develop of themselves into close contact so that the neurokyme flows very directly and with practically no interruption. We shall see as we come further to consider the sympathetic system that this view would account for the nerve action there also.

There is a bit of evidence from another field that has an interesting association with this problem. Greenman (21) has shown that if the peroneal nerve of the albino rat be severed and allowed to heal naturally, the nerve to regenerate, the result is an enormous branching of the nerve at the point of section. He says (p. 63):

"Following the degeneration in the operated nerve, regeneration, accompanied by branches of axons, takes place and there is an increase of from 64 to 249 per cent in the number of fibres on the proximal side of the lesion, more than 7,000 fibres appearing in some cases just proximal to the lesion in a nerve which should show about 2,300 fibres."

This enormous branching was thought to be due to the intrusion of a large mass of connective tissue which seemed to interfere with the easy growth and regeneration of the nerve. The experiment was then tried of surrounding the cut end of the nerve with a celluloid tube, thus protecting it against the interference of the 


\section{ORIGIN OF CONSCIOUSNESS}

connective tissue and allowing it to develop in a simple way. The result here was a great reduction in the branching and consequent number of fibres. The experiment was still in progress at the time of the report. This seems to indicate that operative interference with the natural course of the nerve results in an enormous branching. Just as trees when cut back increase the number of their branches. Of course this is very remote from our problem and is only cited because it helps one to form a picture of the way nerve fibres grow and one may easily conceive that something somewhat analogous may happen in the ordinary development of functions in neurons.

We may therefore conclude from our present knowledge that consciousness is somehow connected with the passage of the neurokyme from one neuron to another and that, since the cerebral cortex is the latest to develop (therefore at any given period most likely to be incompletely developed), and second, since the number of neurons is here inconceivably greater than anywhere else, we may very properly conclude that while we may not say that the cortex is the seat of consciousness, at least it is of vital importance to consciousness.

That consciousness develops and is somehow, somewhere located in the nervous system we know; but if we do not know how or where neither do we know when. It has often been said that we know no consciousness but our own. We think we have good reason for believing other people are conscious as well as ourselves, both because they tell us so and because they act as tho conscious. The latter however is not always a safe basis for the conclusion. A person walking and talking in his sleep may appear fully conscious and, while it may be true that he 
is partially conscious, yet he surely is not so conscious as he seems. Movement cannot be taken as a criterion of consciousness. Consequently when we are considering those who cannot talk and tell us, whether it be the human infant or an animal, we find ourselves on very uncertain ground.

The infant is commonly assumed to be conscious as soon as born but of this there is no certainty. He may be or he may not be. His activities for some time may be pure reflexes. If we could remember our life at that early period we might have some basis for a conclusion, but no one can remember his infancy. There are a few people who can remember as far back as the age of two, but for most persons little is remembered before the age of six, except a few isolated experiences. It may further be urged "if conscious at birth why not before birth?" The question is not so absurd as it at first appears. At the middle of the fifth month of fetal life occurs the phenomenon of the "quickening." This is probably the first reflex. There are still four months or more until birth, during which time nerve fibres are getting medullated and coming to function. At birth there are a few fully medullated cortical fibres and since the moment of birth cannot be considered as producing any sudden change in the amount of medullation, we must assume that there were medullated cortical fibres before birth.

By the foregoing discussion we do not mean to argue either that there is consciousness before birth or that there is none shortly after birth but only to emphasize the fact that no one knows; and secondly to bring out the point that there is clearly a gradual development. Therefore we may safely picture to ourselves a first appearance of a very simple reflex, perhaps at the time of quickening, 


\section{NEURON MATURITY}

and following this a development of ever increasingly complex reflexes: then somewhere in the series, probably not long after birth, the first gleams of consciousness to be followed by a more or less gradual increase in complexity until we arrive at what may be called maturity of consciousness, which may be located at the point of physical maturity or perhaps should be placed at the point of man's highest efficiency just before mental deterioration sets in-the onset of senility.

The parallel development of the nervous system is probably complete. We have seen the beginnings of brain and neuron development and have found that the brain continues to increase in weight until the twentieth year on the average. Beyond this we cannot go and even here we are on uncertain ground. On the one hand there may be a development of function of the brain thruout life, and on the other hand increase of weight does not necessarily mean increase of function. The limit of brain cell development may be reached some years before twenty. On the other hand, one authority claims that neurons may develop until forty-five. However, in the midst of this uncertainty there can be no harm in assuming the age of twenty as the average time for neuron maturity.

When we assume twenty as the average of maturity, we, by the very term average imply that some reach maturity later than twenty and some earlier, indeed we believe that for some it is very much earlier.

Let us turn now to the other side of the picture and see what are the first movements or reactions that we get and how these become complicated as we go up the scale.

The first movement of the human being takes place in utero at about the middle of the uterine period and is known technically as the quickening-the time when the 


\section{PSYCHOLOGY OF THE NORMAL AND SUBNORMAL}

child in the womb first moves. This is probably a reflex movement and may indicate the first moment when the simple reflex arc is complete, the neurons developed and ready for functioning. ${ }^{1}$

At birth we have a great extension of activity usually considered reflex, such as crying, sucking and movements of arms and legs. Then follow grasping, eye co-ordination, turning of head and many other movements all of which lead us to conclude not only that the infant is conscious but that mind is developing.

It is not necessary at this point to enumerate in detail the signs of mental development in the child. They are familiar to all. It is sufficient to note that from these early beginnings there is a constant and regular development up to maturity.

One thing not surely known is whether that development takes place at a constant rate of increase, i.e. whether the child mind normally develops as fast from say age 6 to age 7 as from 2 to 3 : from 11 to 12 as from 8 to 9 . In other words is the curve of mental development to be represented by $\mathrm{A}, \mathrm{B}, \mathrm{C}$, or $\mathrm{D}$, in the accompanying diagram (Fig. 32) ?

We can only say that it is almost certainly not $C$, not likely to be A, and is probably either B or D or some form of the $D$ plan.

We must next try to picture to ourselves how this development of mind is related to the nervous system or,

1 Quickening may of course have no close connection with the development of the nervous system. It may mean that the muscle has become sufficiently developed so that it can contract as the result of a general stimulation, since we know that normal muscle tissue from which the nerve connections have been removed may contract when stimulated eithe: electrically or mechanically. However, it seems rather more natural to conclude that there is a direct nerve connection. These movements of the fetus gradually increase until the time of birth. 


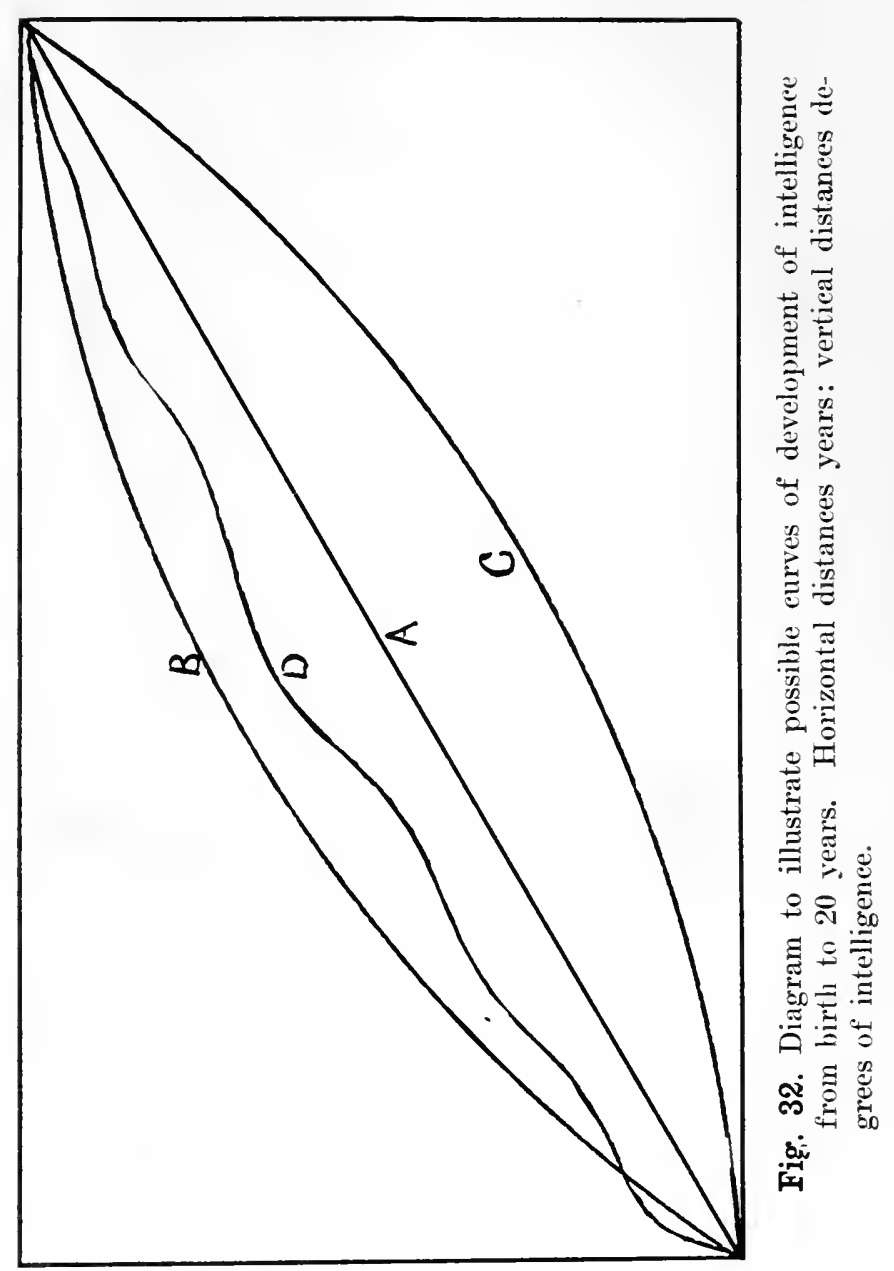





\section{SIMPLE NEURON PATTERNS}

stated differently, what takes place in the nervous system as the physical basis of each step of mental attainment.

We know very accurately, as already explained, what nerve action underlies a simple reflex such as the kneejerk. We have also pictured a somewhat longer nerve path extending to the cortex and we have shown that the cortex has abundant neurons for the most elaborate neuron patterns.

We may now draw some imaginary pictures to help us understand what may take place.

If we wish to attract a baby's attention, we clap our hands or otherwise make a rather loud noise. The air waves-the physical basis of sound-strike the ear drum and by means of the ear mechanism stimulate the auditory nerve; i.e., set into activity one or more sensory neurons. The neurokyme thus started passes to the cortex and into motor neurons leading to muscles that move the eye or turn the head. This is a relatively simple neuron pattern. We may imagine something like Fig. 28, where the energy aroused by the stimulus enters the cortex over the sensory neuron shown at the extreme right (E), and passes across to the series of motor neurons as indicated by the arrows (A, B, C, D). According to the laws of localization, this pattern lies in the auditory centre in the temporal lobe. See Fig. 19, p. 12.)

Again suppose one attracts the child by touching his cheek so that he turns his head. Fig. 29 might be another simple pattern that pictures this process. ${ }^{1}$

Once more, let us assume a bright light flashed in baby's

1 The student must understand that we have represented only a few fibres for the sake of simplicity. It is probable that even the simplest conscious act involves large groups of neurons and the complex processes to be discussed later involve correspondingly larger areas comprising an appreciable part of the $10,000,000,000$ neurons. 


\section{PSYCHOLOGY OF THE NORMAL AND SUBNORMAL}

eyes with his resulting movements to escape the glare. Fig. 30 is a section of the visual cortex and may be taken to illustrate the possible pattern.

These are all simple patterns. We may next consider a more complicated situation.

Suppose a stranger approaches the baby and baby sees him but does not at once move-we say he does not know whether to go to the stranger or to cry. Here apparently is a more complex state of mind and we may picture a neuron pattern involving as complicated a system as that shown in Fig. $31 \mathrm{~A}$ and $\mathrm{B}$.

Finally we may consider a case that will be typical of the higher mental processes. I am writing at my desk when I smell a peculiar odour; it is the odour of burning wool; I remember the fire on the hearth; I recall hearing it snap a few seconds ago; I am sure that a spark has fallen on the carpet and the carpet is burning; I imagine the whole room ablaze; I think of the fire extinguisher and see myself running with it to put out the fire; but the baby is asleep in a far corner of the room, I must carry him to a place of safety before I run for the fire extinguisher; already I imagine I hear him crying and see him squirming in his cradle.

All this "has gone thru my brain" while I am dropping my pen and getting on my feet. What is the neuron pattern? Very complicated indeed. No neurologist could trace it. Fig. 33 is only a diagram to indicate some of the paths and to show by comparison with the previous patterns Figs. 28-31 something of the relative complexities.

It will be noted that in our simpler illustrations the neuron patterns were located in a single cortical area e.g., the auditory centre, the visual, etc., but in this last 


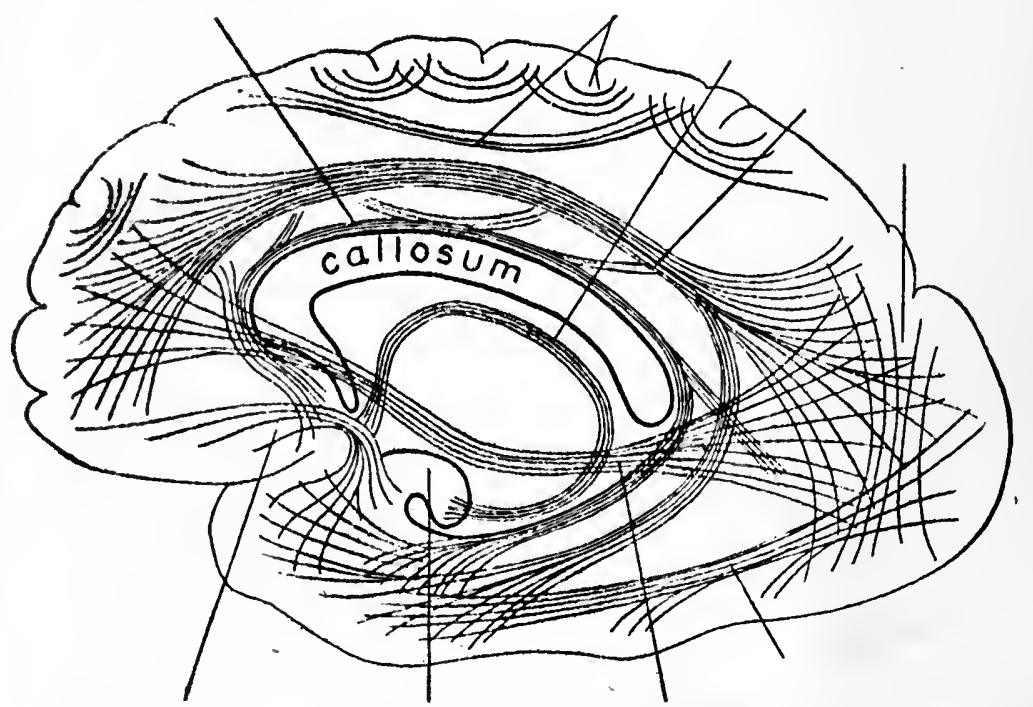

Fig. 33. Diagram illustrating some of the chief association tracts of the cerebral hemisphere. Note how every part is connected with every other part of the brain.

From Herrick 

case all the different centres are involved, including those not yet named. How these get connected we shall now consider.

How did it come about that when I smelled the strange odour I thought of ("saw," had a visual image of) the fire? I smelled the odour (was conscious of an olfactory sensation) because my nose was assailed by a vaporous or gaseous substance that stimulated the sensory neurons of the nose and the energy thus aroused was transmitted to the olfactory centre of the cortex. When I saw "in my mind" the fire, the cells of the occipital lobe were in activity. But those cells were not set into activity by the stimulations of the eye. How then? Before undertaking to answer this question we must consider another topic of fundamental importance.

On page 16 we defined specific stimulus and called attention to the fact that each localized area of the cortex responds to its own specific stimulus. We may now go further and cite the work of Brodmann. He has shown that each area has its peculiar type and arrangement of cells. This still further emphasizes the fact that the cortex has developed for specific functions. It is this idea that we must now extend in order to understand the mental processes that are related to this condition.

It is only a step from the fact of cortical localization (each type of stimulus has its area of the cortex prepared to respond) and Brodmann's discovery of a peculiar type and arrangement of cells in each region of the cortex (special cell structure provided for each special stimulus) to the further fact that there are what we may call specific neuron patterns (certain stimuli find neuron paths already marked out for them).

We have seen, page 20, Fig. 24, that stimulation of the 
patella tendon results in the activity of a simple reflex arc composed of two neurons. Similarly there are definite neuron patterns for the other reflexes.

But we need not stop with reflexes as usually defined. There is a great group of stimuli that are so universally followed by specific reactions that it is certain there is a specific neuron pattern for each. Such patterns give rise to actions that are said to be instinctive. These are the instincts.

Instincts probably differ from reflexes mainly in being more complex-having a more elaborate neuron pattern -and in that they are more or less closely connected with consciousness, perhaps from the fact that their neuron patterns extend into the cerebral cortex. Instinct has been defined by McDougall (34) as "An inherited or innate psycho-physical disposition which determines its possessor to perceive, and pay attention to, objects of a certain class, to experience an emotional excitement of a particular quality upon perceiving such an object, and to act in regard to it in a particular manner, or, at least, to experience an impulse to such action."

We need to emphasize, at the present stage of our discussion, the "innate psycho-physical disposition which determines its possessor" to do certain things, perceive, attend and act or have an impulse to act even if for any reason the act itself does not take place. This is easily translated into the language we have been using. Many of the neuron patterns such as we have described are innate, inherited, that is, they either are ready formed at birth or are potentially there, and do not have to be acquired by any kind of learning process. When the right stimulus is applied the complete reaction takes place and takes place just as it has taken place countless times be- 


\section{INSTINCTS}

fore in the history of the race. So sure is this, that one can predict with practical certainty what will happen. Like a set piece of fireworks the plan is all worked out and only the stimulus is necessary to set it all going.

The number of these patterns is unknown and there has never been any agreement among psychologists as to the number and character of the human instincts. It is agreed in a general way that they cover the necessary activities for the preservation of the individual and the race. Flight, pugnacity, curiosity, repulsion, self-assertion, self-abasement and parental instinct are primary instincts according to McDougall. These we shall discuss later.

We must now emphasize a point already referred to, viz., the late appearance of some instincts. We have already seen that the neurons and consequently the neuron patterns are in a process of development for a number of years after birth-possibly up to adult life. Many simple reflexes and some instincts are fully developed at birth. The rest of the reflexes probably take longer, since these instincts appear only after the lapse of months and even years. This is no contradiction of the fact that they are inborn. The inherited factor is the potential power of the neurons to grow into a predetermined pattern just as e.g., teeth are not present at birth but there are tissue cells with all the inherent prescribed mode of development whereby teeth must appear at the proper time. The same thing is true with instinct. Certain neuron patterns are innate and sure to appear in due time. This leads us to the observation that there are other neuron patterns not innate but acquired.

Thus we come to divide neuron patterns and with them the mental processes into two great groups-the innate 
and the acquired. The former has been called by Thorndike the original nature of man; the latter man's acquisitions. Together they comprise the complete nature of man.

Thus we are brought face to face with the many phased question of heredity and environment-nature and nurture.

The first sign that the new born infant is alive is a cry. This is considered a pure reflex. The change in temperature from the warm body of the mother to the surrounding atmosphere stimulates the nerves of temperature and produces a contraction of the diaphragm and activity of the lungs which produces the cry. No other assumption is possible than that there is at this time a thoroly developed nerve pattern which makes possible the pas. sage of the energy from the stimulus of cold to the contraction of the muscles which means the cry. When the infant is placed to the breast he immediately suckles and here again we have a thoroly formed neuron pattern which is the underlying basis for this reflex or instinctive act. These and many other patterns are well formed at birth, but from this time on new patterns are constantly being formed thru the growth and delevlopment of the neurons necessary to complete the various patterns. The neuron patterns involved in the instincts whatever they may be, or wherever they may be, certainly involve but part of the infant's ten thousand million neurons. What is the function of those neurons not involved in these inherited patterns?

We have said that the neuron pattern underlying an instinct. will be set into activity whenever the appropriate stimulus is applied. There are, therefore, a limited number of stimuli or external influences for which there arc 


\section{ACQUIRED NEURON PATTERNS}

in the human being already prepared receptors; but the number of stimuli to which he is subjected must be infinite. What of that great number of stimuli that do not find in the nervous system a ready prepared pattern? Evidently they must either be without any response, make no impression upon the sensorium, or they must make their own pathway thru the brain, that is, create their own pattern more or less at random. As a matter of fact, both of these things happen. There are many stimuli that so far as we know make no impression whatever upon the sensorium of man. There are wave motions in the air, sound waves, that are so frequent that we never hear the sound. There are wave motions in the ether which we have no eye mechanism to receive. There are many solutions that, applied to the organ of taste, produce no sensation of taste. There are many.vapours that, applied to the olfactory surface, produce no sensation of odour. Indeed it is probably true that only a small fraction of the stimuli that are constantly being applied to us have any effect whatever upon the mind of man.

There are, on the other hand, a great many stimuli that do make their impressions, not by setting into activity any inherited neuron pattern but by creating their own patterns. As I turn my eye in a certain direction the retina is stimulated by rays of light which, for the convenience of the reader, I must describe as dark red, but the single stimulus described would not give me any such reaction. It would indeed stimulate the retina and set into activity certain neurons. But what pattern that energy would follow or what neurons would be stimulated it is impossible to say. According to current psychology it would give me a sensation. It is possible that it would be entirely dissipated in the brain. There are 
many facts which tend to force upon us the conclusion

- that a single stimulus reaching the brain but not finding one of the already formed inherited patterns is lost. A young child makes no response to countless numbers of different stimuli, regardless of their frequency or strength. And it is not difficult to find stimuli that do not provoke a response even in older children. This cannot be due to entire absence of nerve cells since the brains of these children are by no means wanting in neurons and there is every reason to believe that there are, even in quite young children, enough neurons to respond to a large number of stimuli, if a single nonspecific stimulus could produce a response.

If we accept for the time being this view of the case, the next question that arises is, how do these stimuli ever produce a response? The explanation is found in the fact that stimuli do not come singly but in multiples and not thru one sense organ but thru two or more and it is by this means that neuron patterns that fit the child's special environment are built up. How this is done we must now study.

Acquired Neuron Patterns.-In the foregoing discussion it was suggested that the nerve effect of a single stimulus was probably lost in the brain. The more common way of expressing this is to say that the nerve energy started by a single stimulus tends to radiate in all directions, to all parts of the brain, at least to all of the principal localized centres. If the auditory centre is stimulated by a sound wave, some of this energy radiates to the optic centre, some perhaps to the taste and smell centres, some to the kinesthetic or skin centres and perhaps others to the other regions that we have not yet named. But the energy thus divided up is so weakened that it arouses no 


\section{SUMMATION OF STIMULI}

great activity in any one centre and consequently produces no particular result. If we wish to include consciousness in our discussion, we can say, "no definite consciousness in any particular centre." If the optic centre is stimulated by rays of light on the retina, the energy likewise would radiate to all the different centres and become lost. If, however, both centres are stimulated at the same time, we may readily conceive that the line or pathway from the auditory centre to the sight centre and from the sight centre to the auditory centre gets a double stimulation, double activity; the pathway is twice as well worn as in a single instance.

There is a principle in neurology as well as in physics known as the summation of stimuli, a term used to express the fact that a nerve path once having been used, the nerve energy having once passed over a certain line of neurons, those neurons are somehow modified or changed by that passage and the effect is permanent. It is never entirely lost. When a second stimulus comes it not only passes over that line somewhat more easily because the pathway has been used before, but the effect is added to the previous effect, the effects are summed up, and that path has become the favoured path.

If this be true, it is clear that when the eye and the ear are stimulated at the same time the lines of neurons connecting these two centres in the brain will at once have an advantage over any other pathway which has not thus been doubled. If after such an experience the auditor centre alone is stimulated, the energy which was formerly dissipated thruout all of the centres will be now more easily transmitted to the visual centre than elsewhere, with the result that the same centre is stimulated as was formerly stimulated by the visual stimulus. It 
will accordingly produce to a degree the same result as the original stimulus or will arouse the same consciousness.

It is to be expected, however, that while it will produce the same kind of result or arouse the same consciousness it will not produce so great a result or arouse so vivid a consciousness. It is reasonable to expeet that the direct stimulation of the visual centre of the cortex thru its own particular nerve, the optic, would be more vivid, more intense, greater energy would be developed than when the stimulation comes by way of the auditory or some other centre, because in all probability more or less nerve energy is lost in thus transmitting from one centre to the other. Moreover this agrees well with experience.

Considering this matter from the standpoint of consciousness we have the following: My retina is stimulated by the brilliant rays of light and I say, "I see a bright light." If I now see a bright light and hear a loud noise at the same time, this pathway as above described between the auditory and visual centres is deeply worn, speaking figuratively. Now, having had that experience, suppose I again hear the same loud noise, I will at once think of the same bright light that I saw before. But we know that this bright light "thot of," will not be so vivid, so clear or last so long in consciousness or produce the same motor effect as the bright light "actually seen."

It is customary to describe these two experiences by saying that when the centre is directly stimulated by its appropriate stimulus we have a perception of the light, we perceive the light, we see it. But when the centre is stimulated directly from another centre of the brain 

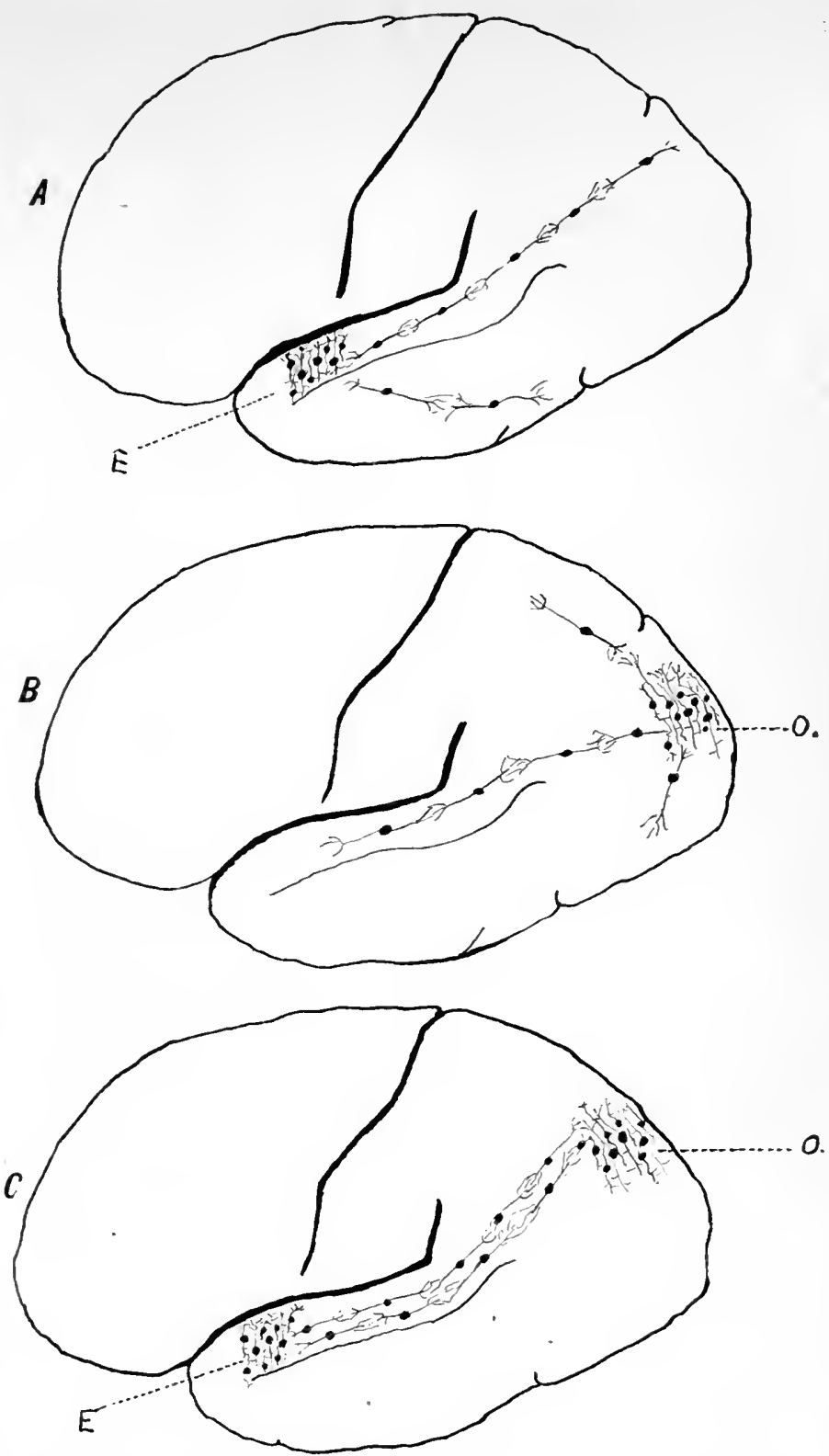

Fig. 34. Diagram to illustrate what happens in brain when ideas are associated. A, an auditory stimulus arouses thru the ear the auditory centre E. The energy radiates in all directions and is dissipated. $\mathrm{B}$, a visual stimulus to the retina arouses the visual centre $O$. The energy radiates in all directions and is dissipated. C, auditory and visual stimuli simultaneously arouse their respective centres. The tract joining them is doubly active. Another time when $\mathrm{E}$ or $\mathrm{O}$ is aroused alone by the external stimulus, the other will be aroused by way of this association path. 



\section{ASSOCIATION OF IDEAS}

we have an idea of the light, a mental picture or a mental image. We say, "There is no light there but I imagine one, or I thot of one." And we know that such an experience is apparently quite different from the direct experience. There is a difference between the real thing and an imaginary thing. The accompanying diagram, Fig. 34, A, B, and C will help to make this clear.

This whole process is commonly called association of ideas, but is in reality, as Titchener points out, not an association of ideas but an association of experiences. I think of the bright light when I hear the sound, or I think of the sound when I see the flash of light, not because I have done anything, not because I will to do so or plan to, but because originally the bright light and the sound were together and they entered my sensorium at the same time, producing the result as above explained.

Extension of the Principle.-In our example we have simplified the matter as much as possible. But if the principle is clear, in the example given, it is easy to extend it in all directions, that is, between all centres of the brain. We are not necessarily confined to two stimuli since there may be more, as many as there are sense organs or receptors.

We thus see that it is a question of what stimuli reach the sensorium and start a neuron pattern; and that wherever two or more stimuli occur at the same time or in immediate succession they become associated, and a neuron pattern is developed. These neuron patterns may, and will, become elaborated by every new experience. For example, the same loud noise that was associated with a bright flash may be followed by other 


\section{PSYCHOLOGY OF THE NORMAL AND SUBNORMAL}

noises of different quality in such rapid succession as to be practically simultaneous. They thus enter into the neuron complex and at the same time the consciousness becomes more elaborate. The bright flash may in the same way be associated with colour stimuli of different sources, until the whole may be grouped together into an elaborate neuron pattern which may underlie in consciousness an elaborate picture, for instance, a man firing a gun.

If all the neurons have developed in a normal way there seems to be no limit to the possibility of such elaboration of neuron patterns. And this elaboration may go on thruout life. The simple pattern is extended by every experience that may later be associated with the original. The man firing a gun may become a Mexican, a Frenchman or a German firing a flintlock, a rifle, a machine gun, a cannon. He may be firing at a target, at an animal, at a man, in war, etc., etc. And thus the mind is built up; and man's acquired nature added to his original nature, the inherited instinct, makes the complete nature of man.

For the sake of clearness we have left out a step in the foregoing discussion. This step is logically important. It seems that we must assume that in the first instance of an association between two stimuli one of them must be specfic; i.e., having its own inborn neuron pattern. Thus the process is a simple extension of the instinctive system. The first acquisitions are thus made by connecting them with original nature. These new acquisitions then become a part of the mind and a basis for any further associations that may be made with it; and so on indefinitely.

If now we consider children in the various stages of [46] 
their development, we find abundant confirmation of this view. Let us take some illustrations. The child sees a man with his head, body, arms and legs. For the sake of simplicity let us assume that each arm and each leg, the head and the body, each is a stimulus; and that they become associated together in the making of the outline of the complete figure. When once that complete association has taken place it is possible for the child, having any one of these applied as a stimulus, to fill out the rest of the picture. For example, thinking of the head he easily imagines the body and the arms and the legs. But we now know with some accuracy that there is a rather definite time when the neuron system has developed to the point where such an association is possible.

It is found by experience and experiment that a child under seven may be given a drawing of a human figure with the arms left out and he cannot imagine the arms. His neuron pattern is incomplete. The different parts of it are so imperfectly associated that the "arm pattern" is not aroused tho all the rest are in activity from direct stimulation by the picture itself. At the age of three his neuron system is apparently very simple. For, when shown a picture, he sees only a few details. He does not associate the different parts together, and name the whole picture. He gives the simplest responses which have been built up by experience. He will point to the man and say, "Man," "Another man." At a little later age he will enumerate everything in the picture but he does not put them all together and tell what is going on in the picture. This may easily be explained in accordance with the above view, that his neuron patterns are not so well elaborated that when a given stim[47] 


\section{PSYCHOLOGY OF THE NORMAL AND SUBNORMAL}

ulus enters the sensorium it arouses to activity the large number of neurons that would mean the consciousness of the picture as a whole. But rather each little detail that catches his eye arouses its little pattern which most frequently involves only the name of the object.

Earl Barnes (3) has shown that the child's first drawing of a man is a circular head; soon he adds eyes, then nose and mouth and finally a body. It is considerably later that he adds clothes, with buttons-and when he discovers buttons he is apt to put them everywhere. Evidently he puts in these various details as he gets conscious of them, as his neuron patterns develop. Altho the whole man has been before him all the time he at first saw only the round face, then eyes. It is of interest to note also that eyes are significant to animals. Wild animals will approach a man if his eyes are closed, when they will not do so if the eyes are open.

In the case of the normal child the neuron patterns are constantly increasing in complexity and in number and the greater the complexity and number, the greater is the capacity for more. Upon this view it is easy to see the fundamental importance of wide experience for the growing child, for it is experience, and experience only that develops the neuron patterns. Even the instincts cannot manifest themselves until their appropriate stimulus is applied. And only the widest possible experience can ever utilize even a part of the large number of neurons that are capable of functioning. If a child were to be shut up and limited to only a few stimuli there would be no possibility of more than a few neuron patterns developing.

The story of Caspar Hauser is familiar. It is supposed that this person had been imprisoned practically 
all his life, about sixteen years, and when he finally escaped and came out into the world he was, to all intents and purposes, an imbecile, if not an idiot. He had been shut away from the thousands and millions of stimuli that should have developed the neuron patterns and consequently he had no capacity at his age for meeting the environment in which he suddenly found himself.

In the feeble-minded we have the same condition brought about thru other factors. The stimuli are indeed present. The feeble-minded child lives in a normal world where the usual stimuli meet him. But because of the failure of a great many of his neurons to develop to complete functioning capacity, he is not able to acquire the neuron patterns which mean adaptation to a large environment.

One of the most noticeable things about a group of idiots is their constant repetition of the same movements; and oftentimes these movements are so individual and peculiar that they attract one's attention. The writer spent months observing this group of defectives and attempting to account for these very peculiar activities. They were so striking that it seemed that they 'must be significant of something much more important than they proved to be. It was thot that there might be traces of a hidden past, in the race if not the individual, "reverberations from the ancestral sea." It was a long time before the simple explanation dawned that they are merely accidental performances, which being once learned the neuron pattern being once formed, the child, not having the capacity for learning new combinations or for developing new patterns, is condemned all his life to go thru with that one activity. For instance, here is a boy that delights to lie on his back 


\section{PSYCHOLOGY OF THE NORMAL AND SUBNORMAL}

on the floor or on a bench, and twirl a string in his fingers above his face. Another one seems to delight to get hold of an old rag and play with it and roll it about and handle it. Another one sits by the hour and moves his foot in a particular way. Many of them sway back and forth constantly. Some utter continually the same sound, the same grunt. Those that approach to the use of language have certain phrases that they constantly repeat. And so, one might go thru a long list. There is no significance to these things, they simply represent the individual's one accomplishment.

As we go up the scale of intelligence we have precisely the same thing except that it is more elaborate. The low grade imbecile has a few more accomplishments and these he repeats continually. The middle and high grade imbeciles can do a little more. The high grade moron can develop quite a good many of these neuron patterns and can learn to do a great many things, but he is never able to adapt himself to a new environment without very special effort on the part of the trainer. Having learned one activity, to do a thing in a particular way, he cannot modify it to suit changed conditions. His neuron pattern is formed and it has little or no connection with any other neuron pattern and consequently there is no change. Even dull normals show the same tendency in their constant use of a few phrases or their repetition of limited activities. Perhaps we need not stop even here!

\section{Summary.}

1. The development of mind follows the development of the nervous system. 


\section{SUMMARY}

2. Somewhere in the course of development consciousness appears.

3. Different parts of the cortex respond to different types of stimuli-localization.

4. The child is born with inherited neuron patterns which need only their specific stimuli to set them into activity. There are also other neuron patterns which while not complete at birth have inherited potentiality for development.

5. The neuron patterns are first simple reflexes, then gradually more and more complex-instinct, perception and idea.

6. To the inherited patterns are gradually added acquired patterns built up by experience.

7. Two stimuli applied at the same time thru different sense organs (e.g., eye and ear) reach their respective cortical areas and become associated-contiguity. Afterwards whenever one is aroused by its appropriate stimulus the other is set into activity by association.

8. The feeble-minded are deficient in the acquired neuron patterns. 


\section{CHAPTER III}

\section{ARRESTED MENTAL DEVELOPMENT}

In the previous chapters has been described the course of brain development including the ever increasing elaboration of neuron patterns. We have traced in a general way the development of mental processes as the nerve cell mechanism becomes elaborated from simple patterns to the most complex imaginable. We have assumed that there is a time when this neuron development becomes complete, at which time there is, so far as the brain is concerned, the possibility of the highest known mental development. Theoretically, every human being has the possibility of reaching this limiting point; but many things may intervene to stop the development of the brain before it has reached this ultimate mature condition. Just as the body itself may cease to develop in stature with the result that we have dwarfs, so we have dwarfed brains with the accompanying dwarfed intelligence.

The feeble-minded have long been described as cases of arrested mental development. They are, it is true, marked examples of the principle, but we wish for the present to consider arrested mental development from a broader standpoint than that of the feeble-minded. For the sake of definiteness we have provisionally taken the arbitrary age of twenty years as the upper limit of development. If, therefore, we assume that the human brain ought to develop, continue to elaborate its neurons and neuron patterns until the age of twenty, at which 


\section{MENTAL AGE}

time we might assume that there is a perfect mechanism developed, it is clear that any brain that ceased to develop prior to that time would be a case of arrested brain development, with its necessary arrest of mentality. Once this concept is grasped, it is easy to see that, as a matter of fact, human society is made up of individuals with every degree of arrested development from those just short of perfect development down to the lowest grade idiot who has only the mentality and practically only the brain growth of the new born infant.

Since we have no unit for measuring mentality, it has become customary to indicate the grade of intelligence by referring to the mental attainment commonly found in children of various ages. For example, a child who has developed normally until the age of eight has what we may call eight year intelligence, the nine year child, nine year intelligence, and so on up and down the scale. According to this hypothesis no one has more than twenty year intelligence since that is the maximum and if all brains developed to the maximum, everybody would have twenty year intelligence, while the person who had only nineteen year intelligence would be arrested mentally and would be one year retarded. Others have stopped with a mentality of eighteen, still others at sixteen and fifteen and at every age, down to one year or even less. On this view it is easy to see that if twenty year intelligence is the maximum, that is to say, represents the mentality of the most intelligent, then the intelligence of the average person would be considerably below this. It is evident that the proportion of people who have exceptionally high intelligence is relatively very small. TTerman (50 p. 140) in his studies of 
this problem has placed the intelligence of the average man at sixteen: this may be not far from correct since Studies of the brain indicate that the neuron development has gone so far by that time that at sixteen years there are enough elaborate neuron patterns to underlie not only the reflexes and instincts but also a great many ac. quired patterns,-sufficient for the ordinary reactions of life.) These, however, might not be sufficiently elaborate to account for a Newton, a Leonardo Da Vinci, a Plato, an Aristotle, a Spencer or the Brownings and all the rest of the great men and women past and present. Just as the average is considerably below the maximum, so there may be many who are below the average. That there are many persons of less than average intelligence is obvious.

A difficulty occurs here, however, which must be explained. It is a common error to think of an intelligence below the average, or even below the maximum, as due to a lack of education. In other words, it is common to confuse intelligence with education. Altho the relation between the two is not easy to explain in all its details, yet they are by no means the same. We can perhaps make the relation fairly clear by a few rather general statements, all of which should be modified to cover the exceptional cases, but which in the main express the relationship. Intelligence is a matter of inborn capacity, of neurons and neuron patterns; education is largely a matter of environment, of opportunity for the exercise of the inborn capacity - the development of elaborate patterns from the neurons that one has.

It is probably true that most people whose short-comings we account for on the ground of lack of education are really lacking in intelligence. Those who have the 


\section{INTELLIGENCE VERSUS KNOWLEDGE}

intelligence as a rule get the education in one way or another,-if not in a formal way thru schools and colleges, then thru the practical affairs of life, such as travel, business, association with people who have education, use of libraries, public lectures, journals, and even newspapers. There are, it is true, many and marked exceptions to this rule. But they are exceptions and in these days of opportunity the exceptions are easily discovered by any one who makes the effort to determine whether such an individual is actually lacking in intelligence or only lacking in knowledge-is ignorant. In the first place it is not at all uncommon to hear the expression, "He is an ignorant man, but very intelligent along his line." In fact, these intelligent ignorant people have usually made a success of life or of some trade or profession-and they are perfectly able to succeed along any line that does not require a wide knowledge and broad education. On the other hand the individual who lacks intelligence or, in terms of our neurological explanation, who lacks the necessary neuron patterns, cannot become educated in any large sense. It is true that he may have a memory for facts and accumulate a large amount of information, but he makes very little practical use of it, and does not show judgment and good sense in the ordinary affairs of life.

The best method of determining who is intelligent and who is merely uneducated is the scientific method of measuring the intelligence. Thanks to the genius of Alfred Binet of Paris, we now have measuring scales for intelligence that determine with a high degree of accuracy the mental level of an individual. Up to the present time these scales are more valuable for the younger years, up to perhaps twelve, altho they are being extended and 
will eventually be applicable to all grades. It is thru the use of these scales for measuring intelligence that we have arrived at the concept of intelligence levels)and have learned that in human beings these lcvels range the entire length of the scale from the intelligence of one year to the upper limit, whether that be twenty years, or more or less.

If now we accept twenty year intelligence as the upper limit and sixteen year, according to Terman, as the average intelligence, we may properly call all those who do not attain to at least the mentality of sixteen as persons who are arrested in their mental development, and in accordance with the teachings of the previous chapters we shall conclude that their neuron patterns are incomplete. The neurons have failed to develop sufficiently to enable the individual to have the necessary patterns.)

It is now a demonstrated fact that many people do not reach the sixteen year level, there are many at fifteen, almost as many at fourteen and thirteen, a great many at twelve and below. Before we can appreciate arrested mental development we must understand normal intelligence better.

What is Intelligence?-In the previous pages we have used the term intelligence in the popular sense as being readily understood by all readers. It now becomes desirable to attempt to define it scientifically in order to be able to speak with greater accuracy of its manifestations and to relate it more closely to neuron development.

We have tried to make clear in the foregoing pages the conception that intelligence is dependent upon and correlative with neuron activity; that the more elaborate and complicated the neuron pattern, the higher the possible intelligence; and conversely, the greater the intelli- 


\section{INTELLIGENCE}

gence displayed, the more elaborate must be the neuron pattern that underlies it; also the opposite, the lower the intelligence the less the neuron patterns are developed.

Inasmuch as intelligence is considered to involve consciousness we may say that intelligence is the summation of consciousness. Conceived as an abstract mental process, it is the sum total of all the related consciousnesses that one is able to bring to bear upon a particular occasion. From the standpoint of activity, intelligent action is action resulting from the most elaborate neuron pattern that is involved in the situation. From the standpoint of efficient living, the intelligent person is one who has had sufficiently good inherited neuron patterns, and has had sufficient experience, to develop an abundance of acquired patterns; and as a result is able at any moment to bring enough of these into connection to enable him to adapt himself to the most complicated situations of life.

Conversely, the relatively unintelligent person is one who is unable to adapt himself to new conditions, either because he has not inherited the necessary neurons, or because the conditions of his life have been so unusual and abnormal that he has not had the experience to bring his neurons into co-operation and thus develop sufficiently elaborate patterns. The latter condition, sometimes called defect by deprivation, is so rare that the few instances of it are usually marked cases, such as that of Caspar Hauser already referred to or cases of the deaf and blind who have not been specially educated. We may easily conceive that Helen Keller and Laura Bridgman would have been of low intelligence had not special means been used to reach the brain and develop neuron patterns thru the senses that remained intact.

The Feeble-Minded.-In our discussion of intelligence 


\section{PSYCHOLOGY OF THE NORMAL AND SUBNORMAL}

we have used the expression "ability to adapt one's self to one's environment or to new situations." We may quote Stern who defines intelligence as "the general capacity of an individual consciously to adjust his thinking to new requirements." According to this, intelligence consists in a general adaptability to new problems and conditions of life. It will be seen at once that this adaptability is a good measure of intelligence, and those people who are best able to adapt their thinking and their actions to the most elaborate situations are the most intelligent; while those of less intelligence, altho able to adapt themselves to the ordinary conditions of life, are confused and rendered helpless by the more complex situations. Still lower: there are those of whom James says that they are "nonplussed by all but the very simplest situations."

Now the ability to live an independent existence, to make a living, and to conduct one's self with reasonable propriety in a modern community involves a certain amount of this power of adaptation and there is a certain minimum below which one cannot succeed. That point is usually taken as marking the line between the normal individual and the feeble-minded.

Tredgold's definitions are based upon this principle. $\mathrm{He}$ defines feeble-mindedness in the generic sense (Amentia) as:

"A state of mental defect from birth, or from an early age, due to incomplete cerebral development, in consequence of which the person affected is unable to perform his duties as a member of society in the position of life to which he is born." He describes a high grade feebleminded, now called a moron, as :

"One who is capable of earning a living under favor- 


\section{FEEBLE-MINDEDNESS}

able circumstances, but is incapable, from mental defect existing from birth, or from an early age, (a) of competing on equal terms with his normal brothers; or (b) of managing himself and his affairs with ordinary prudence." From the examination of hundreds of children with the earlier forms of the measuring scales of intelligence it was found that no inmates of institutions for the feeble-minded had an intelligence above twelve years,except a few who had been placed in the institution mainly for delinquency and whose ability otherwise to get along in the world was demonstrated. From this it has come about that twelve year intelligence is usually considered the upper limit of feeble-mindedness or the lower limit of normality.

As a matter of fact it is impossible to draw any hard and fast line. That is evident from the definitions of Tredgold already quoted; since it is clear that some environments require much higher intelligence than others and it is common to say that people who could get along fairly well in the world a hundred years ago when conditions were much simpler would be utterly incapable in the present day and would therefore be considered, according to the definition, as feeble-minded.

Perhaps more careful study may eventually show that the line is to be drawn at the beginning of the adolescent period and that those persons who are arrested in their brain development previous to the onset of puberty must be considered mentally defective, while those whose development goes on into the adolescent period, but stops before its completion, will range from a dull mentality (the so called dull normal person) up to a level of considerable intelligence but short of complete development, and only those whose mental growth (the elaboration of 


\section{PSYCHOLOGY OF THE NORMAL AND SUBNORMAL}

whose neuron patterns continues thru the adolescent period will be capable of the highest intelligence. This latter point brings us back to our hypothetical assumption of twenty years as the limit of brain development. If this view should prove to be a fact it would emphasize the importance of what Dr. Crampton calls physiological age. This principle of physiological age, the fact that children vary widely in the time when new physiological functions such as those of puberty develop, has not been made use of to the extent that it deserves.

Going down the scale of mental development it has become customary to call those mental defectives who have a mentality of from eight to twelve years, morons; those who range from three to seven, imbeciles; and those of two or under, idiots.

In the foregoing we have been speaking of cases of arrested development, and have stated that it is now possible to ascertain the mental age or intelligence level of any person. Our next problem is, given the intelligence level of a person, how do we know that his development has absolutely ceased? In answer, two things must be noted: First, theoretically we can never be certain from a singe examination that the person is arrested in his development. He may be slowing down but not yet stopped. The smallest amount of data from which we could draw a conclusion would be two examinations sufficiently far apart to warrant us in concluding that there should have been development; and if the examination shows none, we may conclude that complete arrest has taken place. But secondly, experience with such people has enabled us to make more or less probable guesses.

Binet says that for children under nine years of age, those whose mental age is found to be two years below

[60] 


\section{ARREST OF DEVELOPMENT}

their chroisological age are to be considered mentally defective. Those nine years and older must be three years backward before it is safe to draw that conclusion. Experience has abundantly proved that Binet was well within the facts in his rules. But care must be taken as to what inferences are drawn from this conclusion. Binet's dictum implies nothing as to whether the individual has come to complete arrest. As stated, experience has proved that such persons will be mentally defective; but it does not show that they will never have a higher mental age than at the time they are tested. In other words, one examination, tho it shows that a child is more than two or three years backward, does not yet show us that he has come to an absolute arrest, but only that he is so far backward that he will ultimately be arrested in development. We may have caught the child when he is slowing down but has not yet stopped.

Again experience has proved that these cases have a slowing down period of probably from one to three years. Moreover the slowing down process may begin at any time during childhood. The exact limits are as yet unknown. Of course, with the idiot the matter is relatively quickly settled; with the imbecile less quickly and with the moron it becomes a problem for a number of years. Some morons are apparently normal in development until five or six years of age, possibly up to seven or eight, and then have a period of three or four years of slowing down until they come to complete arrest. A child now in the Vineland Training School admitted at the age of two, showed at that time a normal mentality of 2 , at the age of two and a half he tested 2.5; at the age of three years nine months he tested 4.4, but at the age of five he tested 4.2. At five 


\section{PSYCHOLOGY OF THE NORMAL AND SUBNORMAL}

and a half he tested 4.8. Now at the age of six and a half he tests 4.6. We give the exact results of the test. It is not probable that he gained and lost as the figures would seem to indicate. A single test question accidentally gained or lost would cause the difference. It is clear that he is slowing down and if one dared predict it seems probable that he will come to complete arrest somewhere around eight years.

It does not appear likely that all such defectives have the same rate of slowing down; some probably reach the stopping point more quickly than others, just as some begin the slowing down process at an earlier age than others. In Fig. 35 is shown the curve of the above case (D) and also several others showing similar slowing down but at different ages and rates. Also it must be noted that the path by which the child comes to his complete arrest of development is dependent upon the cause of the condition. The above is a case of hereditary feeble-mindedness and what has been said has been with the hereditary feeble-minded in view. In the cases of disease or injury there is a different state of affairs. The child who becomes feeble-minded from a fall or an injury to the head would of course stop development almost at once, as would probably those cases that are the result of disease such as cerebro-spinal meningitis.

The condition of neurons in the feeble-minded and other cases of arrested mental development.

It will further elucidate both the problem of arrested development and the neuron basis of mind to consider briefly the brain condition in cases of arrested development. The study of the feeble-minded, while still very incomplete, has given us some very important data bearing upon this topic. First, in gross anatomy, the brain 


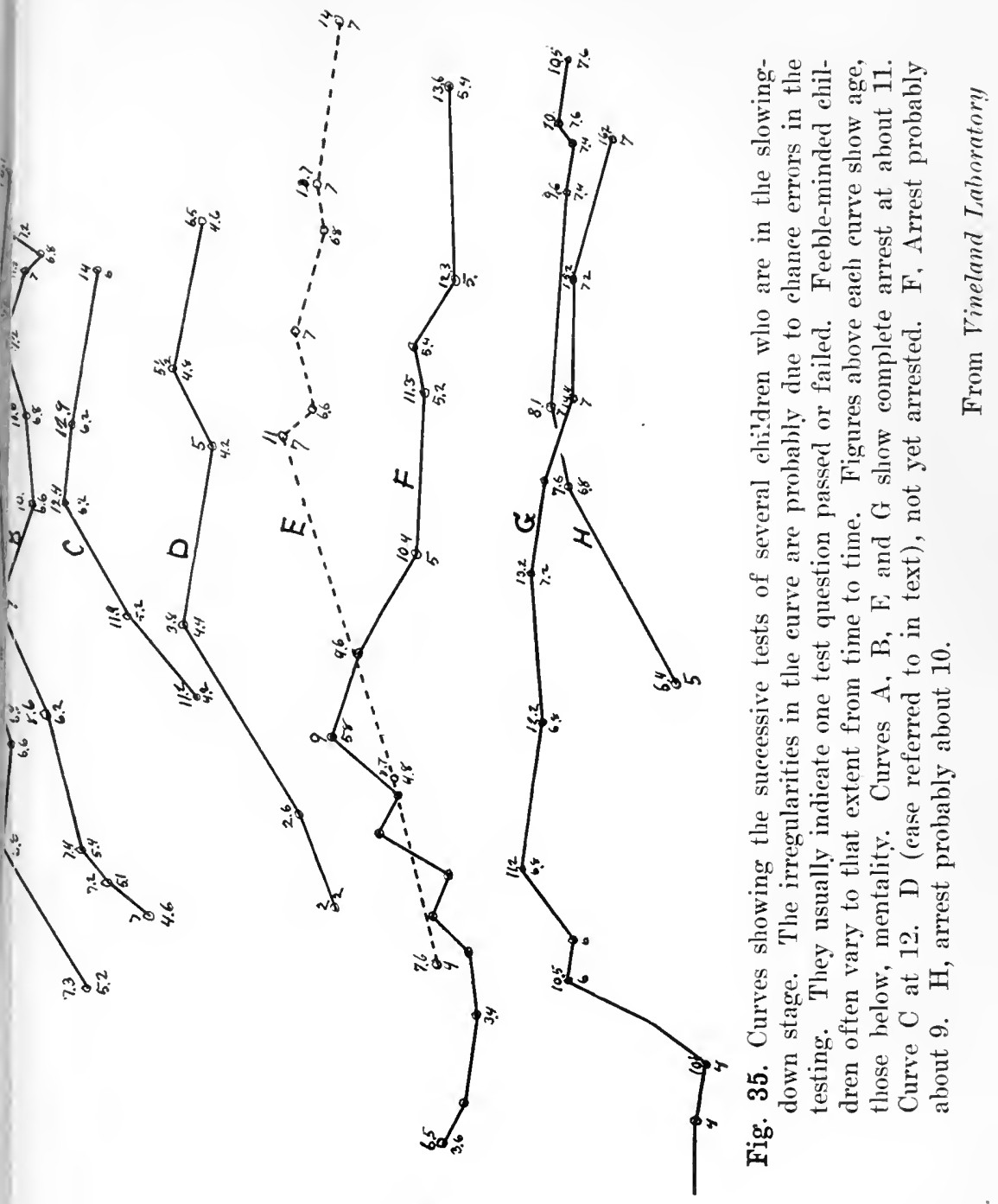



of a case of arrested development does not usually differ from the normal brain; size and shape of head have as a rule no correlation with arrest of development. There is one marked exception, a type of mental defective known as microcephalic (small headed); they are, however, so few as to be marked cases in any group of defectives.

Even the external appearance of the brain itself shows no condition characteristic of feeble-mindedness. The number of convolutions is not markedly different from the normal. Indeed, of a hundred brains, half of which would be the brains of mental defectives, ranging from idiocy up, it is probable that no neurologist, however familiar with brain convolutions, would be able to group the brains accurately, not even those of the idiots. We do not mean that many idiot brains are not markedly "defective," and unmistakably abnormal, but we mean to say that many an idiot brain is in outward appearance well within the limits of variation found in normal brains; and the more experience one has had with normal brains the less likely he is to feel sure that a certain brain that is more or less atypical is the brain of an abnormal person. Compare the two brains here pictured (Fig. 36).

This only means that the difference between the brain of a case of mental arrest and that of a normal person, for there is a difference, is to be sought, not in the gross structure but in the finer microscopic condition and possibly even only in the chemistry of the neuron. Unfortunately there has been as yet very little comparative study of the brains of defectives and normals.

Hammarberg (22), who made one of the earliest and most careful studies of the brains of defectives, found 


\section{PSYCHOLOGY OF THE NORMAL AND SUBNORMAL}

some differences that are classical, but unfortunately the number of brains upon which he worked are too few and too heterogeneous for safe conclusions. His study is based upon the investigation of four idiots, two low grade and three middle to high grade imbeciles. Of these one idiot was only thirty-two months old at death, one "low grade imbecile" twenty months, one idiot three years, the rest twelve to twenty-six years. One low grade imbecile was an epileptic. Several were clearly cases of disease.

The accompanying picture, Fig. 37, shows the difference in cell bodies as Hammarberg found them. He concluded that in his idiots development of neurons had ceased between six and nine months of fetal life; and in the imbeciles during either late fetal life or first year after birth. When it comes to the brains of morons, no study has yet shown any difference between these brains and the brains of normals. This is undoubtedly due to the fact that we have as yet no adequate technique for detecting the differences, since we have every reason to infer from the psychological conditions distinct neurological differences. We shall take up these points more in detail in later chapters, but it will be of interest and value to point out here the general conclusions as they have been worked out, especially since they illustrate the principle of brain development, neuron growth and neuron patterns.

From what has already been said, it will be clear that the degree of mentality or the mental level in any case of arrested mental development will depend upon the time when that arrest occurs, that is, at what stage of development of the neurons the arrest takes place.

Children whose neuron development ceases while only a few of the neurons are mature will not have the mechan- 


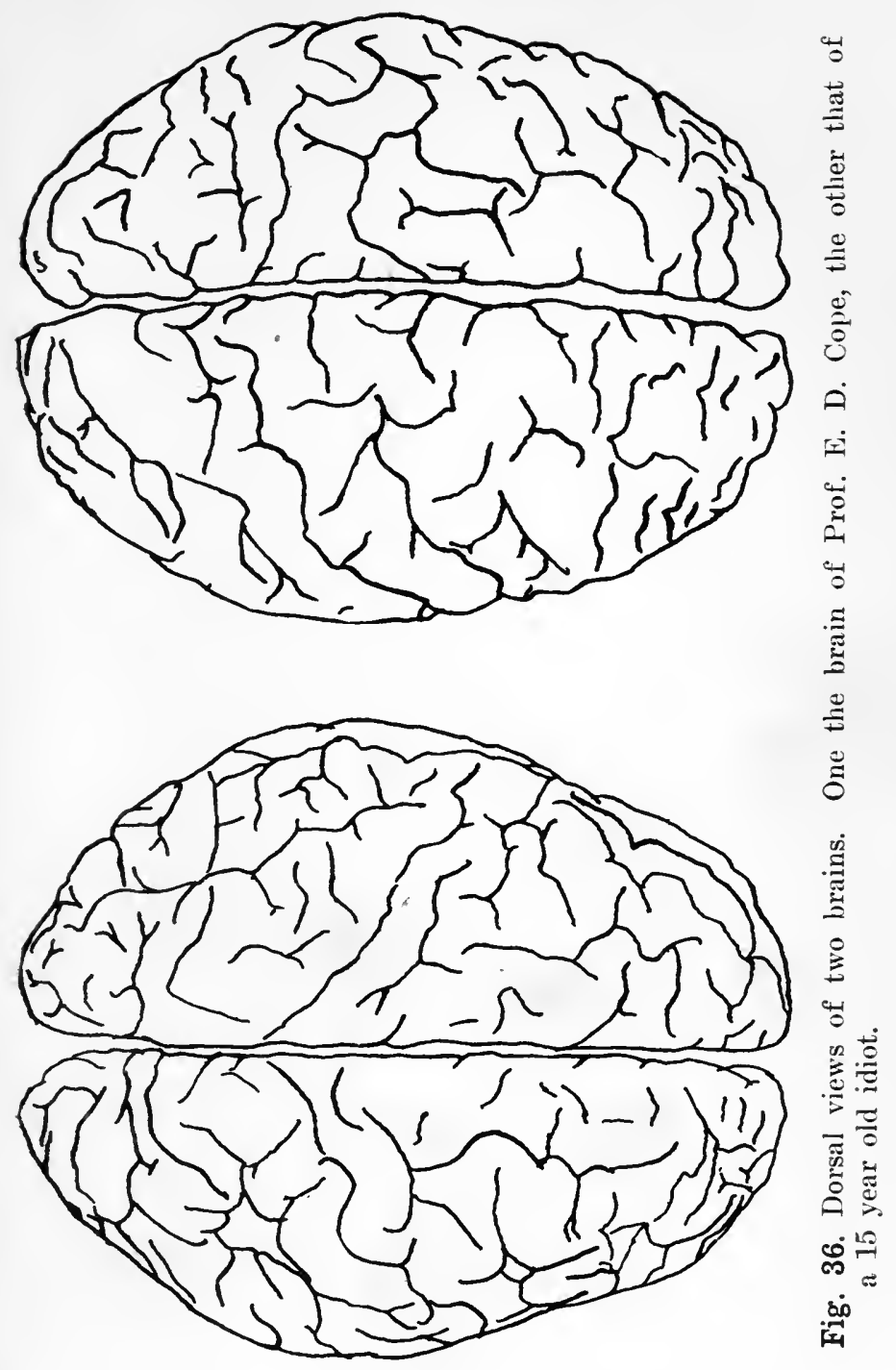



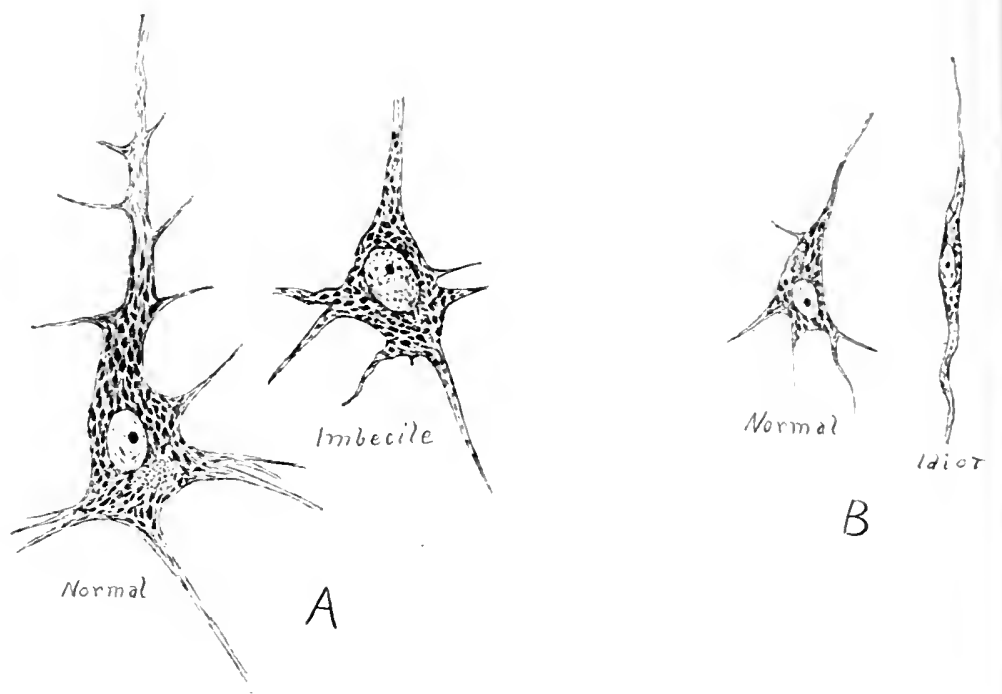

B
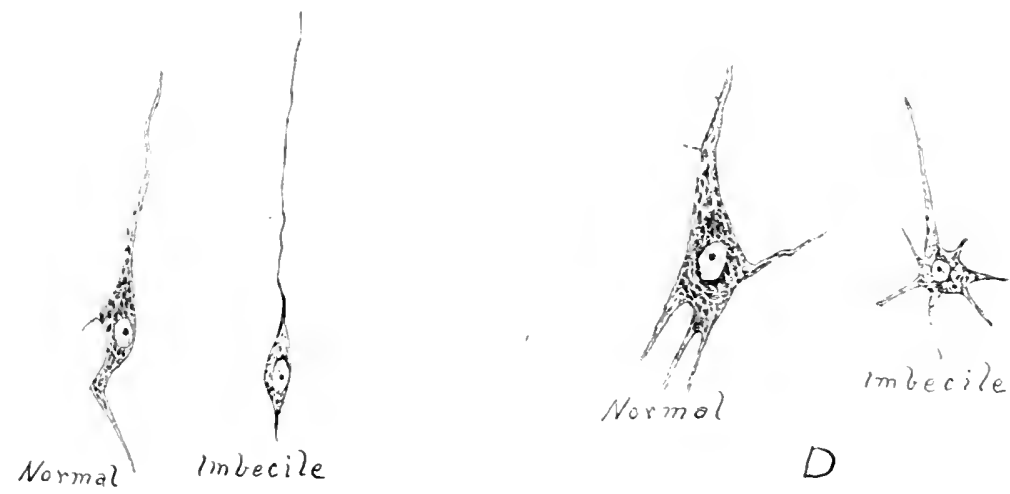

Normal

$D$

C

Fig. 37. Comparison of four types of brain cells from a normal man 28 years old with similar cells from imbeciles and a 14 -year idiut. Note relative size-all are magnitied in the same proportion-and number of processes. A. Giant cells from gyrus centrulis anterior. B. Pyramid cells from gyrus frontalis. C. Spindle cells from gyrus centralis. D. Ganghon cells from gyrus occipitulis superior. From llammarbery 


\section{ASSOCIATION CENTERS}

ism necessary for a high grade of mentality, while those whose arrest occurs at a time when the neurons are almost completely matured will lack only a little of normal mentality. Fortunately we know something of the development of the brain at various ages of childhood. The spinal cord, and what is sometimes called the brain stem (the medulla, the cerebellum and the basal ganglia, all that develops from the primitive cord except the very anterior portion), are well developed at birth; and Flechsig (16) has shown (Fig. 22) that there are even some medullated, mature fibres in the cerebrum itself and that others develop very rapidly in the first few weeks or months. This means that the reflexes and many if not most of the instincts are functioning at birth. It follows from this that we have to do mostly with the cerebral cortex in considering the physical condition of various stages of mental arrest.

Turning to the cortex, we find that the localized centres as described on page (12) seem to be fairly well developed even in cases of early arrest; that is, low grade defectives, even idiots, see and hear, taste and smell, with approximately normal capacity. It is true that they seem to be somewhat dull in taste and smell, but that may easily be because there is an intelligence factor in normal capacity in these lines, involving the higher centres and that the pure sensation is as strong in these cases as in normal people.

In our chart of brain localization (Fig. 19, p. 12), we found that there were large areas where no mental function had been located. These have been called by Flechsig association centres, and have long been considered the seat of the so called higher mental processes. On the authority of those who have studied these regions of the 


\section{PSYCHOLOGY OF THE NORMAL AND SUBNORMAL}

cortex most carefully, we are safe in assuming that they contain vast numbers of association fibres whose function is to bring into relation all the other centres that record sensations and originate muscular movement.

Herrick (23, p. 290) says: "It must be borne in mind that the most significant parts of the human cerebral cortex are the association centres. These alone are greatly enlarged in the human brain as compared with those of the higher apes. In the latter animals the projection centres are fully as large as those of man, the much smaller brain weight being chiefly due to the relatively poor development of the association centres."

Bolton (7) says: "The frontal association centre is the last part of the cerebrum to be developed, and is the first to undergo dissolution; it is under-developed in amentia of all grades, and atrophied in dementia according to its degree."

James has said that one difference between man and the lower animals is that the lower animals take in and give out, while man takes in, works over, and gives out; which means that in the animals, action is largely reflex or akin to reflex, that is to say, energy coming in over a sensory neuron passes out directly over a motor neuron; whereas in man sensation coming in over a sensory neuron may pass thru these association centres, thus developing the elaborate neuron patterns already described, before it passes out and results in motion. From this it would follow that persons in whom these centres were not developed would be incapable of the working-over process, or of making the associations referred to. Their mentality would be only such as could be carried on by simpler neuron patterns; and the elaborate patterns 
which are assumed to underlie deep thot, abstract principles, careful judgment and logical reasoning are impossible.

Referring again to Flechsig and other students we find that these association centres are, as a matter of fact, the last to develop. We may therefore complete our picture of brain development and brain functioning by imagining that arrest of development at various ages of childhood finds these association centres correspondingly incomplete. The details of this must be worked out in connection with later topics, but it is important at this time to grasp the concept of the limitation of brain patterns owing to incomplete development of these centres, which leads us to the very important point of view that cases of arrested development who lack judgment, reasoning power and volition are not simply cases of lack of education but are persons of little judgment because they have not the developed brain cells necessary for the elaboration of mental processes sufficient to give the judgment.

We are now ready to take up the consideration of various mental processes. We shall note the development of those processes in various degrees of mental defect and try to picture to ourselves the reason for that defect in the condition of the neurons.

\section{Summary.}

1. The growth of neurons often stops before complete development is reached, giving rise to mental defectives.

2. Society is made up of persons of all grades of intelligence.

3. Mental defectives are not simply ignorant. They either lack neuron development or-in rare instances- 
have not had the proper stimuli to arouse their neuron patterns-defectives by deprivation.

4. If mental arrest occurs at twelve years or lessperhaps before the beginning of puberty-the person is feeble-minded.

5. The feeble-minded are divided into idiots, imbeciles and morons.

6. The mental level can be determined by suitable tests.

7. In the feeble-minded the three "association areas" are probably the parts of the brain most deficient in growth. Other parts of brain and nervous system are relatively little affected except in very low grades.

8. Since the higher mental processes depend upon the association areas, the cause of feeble-mindedness may be sought in the defective development of these areas. 


\section{CHAPTER IV}

\section{SOME PROPERTIES INHERENT IN THE NERVOUS MECHANISM}

Any mechanism as elaborate as the nervous system has some properties inherent in its very nature. These properties must now be considered because they are fundamental for the beginnings of mind.

First we note that out of this mechanism, consciousness arises. No one knows just how this comes about; but we have tried by more or less plausible hypotheses to give the reader a working plan of how it might be, so that he may have something concrete and tangible to think about. We have assumed that there are neuron patterns, some comparatively simple and some exceedingly complex, with all gradations between. Just as the child starts in life with muscles ready to contract, digestive organs ready to function, and many other mechanisms ready to work, so he has numerous neuron patterns fully developed needing only the proper stimulus to arouse them to action. These neurons have certain properties and are organized to work in definite ways.

At least one mental process is the direct consequence of the nature of the neuron and at least two fundamental processes are the natural consequences of the fact that neurons are organized into patterns.

Memory.-Of the properties inherent in nervous tissue, we shall consider irritability because this is the basis of memory. Irritability is that property of living matter by which it responds to a stimulus. Nervous tissue is 


\section{PSYCHOLOGY OF THE NORMAL AND SUBNORMAL}

probably the most high organized substance in the world. Therefore we have a right to assume it is the most irritable-responds most delicately and characteristically to stimulation. A further consequence must be that in such a highly organized substance, stimulation must produce not only a temporary response but a more or less permanent effect.

Herrick (23) (p. 294-5) says: "All functions of the nervous system are facilitated by repetition, and many such repetitions lead to an enduring change in the mode of response to stimulation which may be called physiological habit. This implies that the performance of every reaction leaves some sort of residual change in the structure of the neuron systems involved. These acquired modifications of behaviour are manifested in some degree by all organisms, and this capacity lies at the basis of all associative memory (whether consciously or unconsciously performed) and the capacity of learning by experience. This modifiability thru individual experience is possessed by the cerebral cortex in higher degree than by any other part of the nervous system; and the capacity for reacting to stimuli in terms of past experience as well as of the present situation lies at the basis of that docility and intelligent adaptation of means to ends which are characteristic of the higher mammals."

When a stimulus is applied and neurokyme flows over a given line of neurons, those neurons are somehow changed, and changed more or less permanently. Whether this is the result of a change in their chemical composition or in their molecular arrangement we can only guess. This condition, however, is not without analogies in other realms of nature. For example, when a piece of steel is heated and cooled it is found to be radi- 


\section{INHERENT MEMORY}

cally changed. Whereas before heating it was elastic and springy, after heating it is pliable and ductile. Again, when a steel rod or wire has had a current of electricity sent through it in a definite way, it is changed. It has become magnetized. In this case the change is supposed to be due to a change in the molecular arrangement.

To return to our nerve cells, the important fact for present consideration is that the change in the condition of the neuron shows in consciousness when the stimulus is again applied. The neurokyme appears to flow under changed conditions. Perhaps it flows faster; perhaps slower; it may be with greater difficulty or possibly with greater ease. The new element in consciousness, usually described as a feeling of familiarity, results in a recognition of the fact that we have had this experience before, and we say we remember it. Memory as thus described is therefore a property of nervous tissue.

The first time a loud sound strikes the baby's ear it arouses the inherited neuron pattern in the auditory centre and he has, as we have explained, a sensation of sound. The next time the same stimulus is applied it comes with that changed condition which gives the new element of consciousness that we call the feeling of familiarity, and the baby is conscious that he has heard that sound before.

Because of this property, any neuron pattern once aroused by a given stimulus tends to be aroused in the same way again, giving rise to the same consciousness with the added feeling of familiarity, in which case the stimulus is remembered. This may be called inherent memory-inherent in the nature of nervous substance. It is also called natural retentiveness.

Wherever there are neuron patterns, be they simple or [71] 


\section{PSYCHOLOGY OF THE NORMAL AND SUBNORMAL}

elaborate, there will be this memory. It is not subject to training or education. It is not influenced by intelligence. It may be a factor in intelligence since it enables one to recall many experiences, and experiences are of fundamental importance for intelligence. Good natural retentiveness means that every neuron pattern that has been in activity may be aroused again in the same way. Poor natural retentiveness means that the nervous tissue is of such quality that the impression is not retained in all cases. Disease and fatigue may affect the condition of the nervous tissue and thus interfere with its retentiveness, but among healthy persons there is apparently very little difference in the quality of the nervous tissue hence very little difference in the capacity for retentiveness.

What is thot to be poor memory is first, poor associative memory which will be considered later; and second, poor stimulation. The experience that we do not remember was never adequately in consciousnessthe neuron pattern was not fully aroused or was not aroused with sufficient energy; to revert to our illustration of the magnetized iron, the magnetism is weak because the electric current sent thru it was not of sufficient strength.

Imagination. The feeling of familiarity may be lost. The neuron pattern if not soon aroused again seems to lose the impression originally made and when finally aroused, the same consciousness results but without the added feeling of familiarity; hence the experience is not remembered.

Imagination is literally imaging, and the image has been defined by Binet as "a phenomenon which results from an excitation of the cortex." This makes it cover 


\section{IMAGINATION}

the two concepts of perception and idea. It has more often been considered synonymous with idea. That is, we use the term mental image as descriptive of a simple state of consciousness aroused, not directly by an external stimulus, but indirectly from another centre of the brain. For instance, at the present moment $I$ have a mental image (visual) of a building in another city. I have a mental image (auditory) of Lohengrin's Wedding March; I have a mental image (olfactory) of the odour of coffee, of the taste of sugar (gustatory), of a caterpillar crawling over my neck (tactual). All of these are, of course, previous experiences revived, and run so closely into memory that it is possible to describe all of them by saying I remember them.

It has been said that imagination is memory minus the date, that is to say, the image is not referred to a definite past experience. The image of the aroma of coffee is not the image of the coffee that I had for breakfast this morning, or on any other particular occasion, but just the aroma of coffee without being located in any past time; likewise my image of a building in a distant city. I may be conscious that I have seen such a building, but I cannot tell when and indeed I may be forced to say that I am not sure that $I$ have ever seen it. But if my image is reasonably true to the facts, it is certain that $I$ have at some time actually had the experience.

In other words, all these cases are simply instances of an old neuron pattern revived, either by word or by association with something else that is now present in experience. This kind of imagination is called reproductive imagination. I have simply reproduced an old experience and as already stated, if that old experience is definitely located in past time, I properly say I remember 


\section{PSYCHOLOGY OF THE NORMAL AND SUBNORMAL}

it. But if the fact of its having been experienced has been lost, and the image comes into my mind without being definitely located in time, I call the experience reproductive imagination.

Such imagination involves only simple neuron patterns and therefore is possible for undeveloped minds and even for animals. Any neuron pattern once developed may be revived at any time, and may be revived under such circumstances that it is not located in time or place. This, however, is not what is popularly considered imagination. This is imagination in the psychological sense of a reproduced image.

Every one knows that children have both memory and imagination, but the reader may be surprised, as are many people, to learn that the feeble-minded also have both memory and imagination; and yet it is clear that, as only simple neuron patterns of the inherited type are involved, there is no reason why defectives who have any neuron patterns at all should not have both memory and imagination as we have so far described them. It is very common to hear teachers or parents question the diagnosis of feeble-mindedness for any particular child, because he has a remarkable memory. But any one who has become familiar thru experience with feeble-minded children, knows that this is not incompatible with feeble-mindedness. Indeed there are occasionally truly remarkable feats of memory among the feeble-minded. The writer spent a day in an institution for the feeble-minded several years ago. Two years later he returned for another visit and was amazed to find several of the children remembered him and called him by name. Marjorie B. was born in China. She can recite to you a long story about her birthplace and her family. Robert was very fond of recitation 
and learned a great many selections. He never forgot them, could at any time repeat any selection that was called for. Feeble-minded children are very fond of music and in one school the children are taught all the hymns and new songs. As new ones are learned, the old ones are not sung. A few years ago the teacher of music was asked to find out how many of the old songs the children could still sing. Accordingly, some weeks were devoted to singing the old songs. More than two hundred were thus revived, many of which the leader himself had long since forgotten. Not only were there children who remembered the words, but there were always some who could remember the tune.

"Fire-alarm Joe" remembered the exact dates of all the fires that had occurred within a certain radius of his home in the city, could tell just what engines responded, and other facts about each fire. Occasionally one finds in some of these institutions a child that remembers birthdays. He asks the birth date of everybody that he meets and then never forgets it.

Even idiots very clearly show memory, tho of course, for very simple things. In the nature of the case it is not so easy to prove that they have imagination, since the only difference between reproductive imagination, of which we have thus far spoken, and memory is the date; and it requires introspection to tell whether the date has been lost or not. An observer is unable to satisfy himself whether it is imagination or memory. There are, however, instances where children repeat an action that they have performed before and yet very evidently have no conscious memory of the previous experience.

The explanation of the remarkable feats of memory of the feeble-minded seems to be that, since, as we shall 


\section{PSYCHOLOGY OF THE NORMAL AND SUBNORMAL}

see later, they do not have the elaborate associations that normal people have, the entire nerve energy must run in a few patterns, thus keeping them all active. The business man does not remember all the fire alarms because so many other stimuli that arouse more elaborate patterns come to him. It is sometimes said that modern man is losing the power of memory and in proof certain wonderful memory feats of the ancients are cited. This is simply an illusion. In ancient times there were no telephones, telegraph, airplanes, or railroad trains. The brains of the ancients did not have to respond to so many stimuli, hence what did come to them could be lived over and remembered. Today people who live in isolated communities remember quantities of details that the man of affairs forgets because his mind is occupied with other things.

We have seen that memory and imagination in their simple forms are mental processes resulting from, or inherent in, the nature of nervous tissue. We next consider those processes that are inherent in the nature of the mechanism of the nervous system-the fact that there are neurons and synapses, neurokyme, stimuli and muscles.

Attention.-We have made much of nerve action-the neurokyme flowing thru the neuron patterns. This action when sufficiently complex results in consciousness. It is a logical deduction that the more complex patterns give rise to larger and more intense consciousness; and further, that if a number of these patterns happen to be aroused at the same time there would generally be some stimulus so much stronger than the rest as to arouse a more intense consciousness and thus obscure the others, and we should be conscious of that stimulus to the more 


\section{ATTENTION}

or less complete neglect of the others. This is attention; and it is customary to say that we are attending to that particular stimulus; or that we are in a state of attention. It is common to define attention as the focus or the focusing of consciousness. We prefer to describe it as above, and thus avoid the implication that " $w e$ " do any focusing or concentrating or attending. The stimulus arouses the consciousness and that consciousness is the attention.

Which one of the many stimuli constantly reaching the brain will arouse the stronger consciousness and therefore "hold the attention" depends upon first, the strength of the stimulus and, second, the degree of ease with which the nerve energy flows into an elaborate neuron pattern.

Other things being equal, the stronger stimulus will arouse the greater consciousness and we attend to that stimulus. On the other hand the inherited neuron patterns, being ready prepared to receive their specific stimuli, will in general respond more easily ${ }^{1}$ than acquired patterns. Therefore, "we attend" to those stimuli for which we have an inherited pattern to the exclusion of those stimuli for which we have only an acquired pattern. Nevertheless, it is probably true that an acquired pattern may, by use, become so easy of arousal that the consciousness accompanying it becomes even greater than that of an inherited pattern-especially if, on the one hand, the latter has been relatively little used,

1 "Ease of flow" of the neurokyme is a convenient concept to explain the changes in consciousness. It must be kept in mind, however, that we really know nothing of the actual changes in the transmission of energy thru the neurons or even whether the difference is in the neurons or at the synapses. In another place we have suggested the latter. Moreover, if for convenience we adopt the concept of degree of ease of flow, it must be remembered that the degree that means the acme of consciousness is perhaps not the greatest ease, since we also conceive that when the neurokyme flows with the highest degree of ease, there being no resistance, there is no consciousness. 


\section{PSYCHOLOGY OF THE NORMAL AND SUBNORMAL}

or on the other, has been used so much that the stimulus leads to its natural action with so little interference that a minimum of consciousness is aroused.

We call the state of attention that results from the activity of an inherited neuron pattern, instinctive or inherited attention and that which comes from an acquired pattern, acquired attention. The former has usually been called involuntary or passive attention and the latter, voluntary or active attention. We prefer our terminology because the other implies a directing force for the attention which is a purely gratuitous assumption and leads to much confusion. According to this view, attention is only a name for the maximum consciousness at any given moment and is dependent upon the character of the stimulus and the neuron pattern.

There apparently may also be a second centre in activity, giving rise to a vague consciousness which we recognize as a marginal consciousness. There may even be an alternation between these two, now one predominating and now another. Then we say our attention fluctuates, as for instance when one tries to listen to a friend and also think what one will say in reply, or when one tries to attend to the task in hand but some other matter "persists in forcing itself on the attention."

Immature mind is capable of instinctive attention. The lowest idiot, like the normal infant, turns his head at a loud sound or a bright light. Of course it is impossible always to say that such movements are not reflex, but if conscious in the normal infant they are probably so in the idiot. Moreover, the range of stimuli to which defectives attend gradually increases as we ascend the intelligence scale and parallels more or less closely the development of the same process in the growing normal 


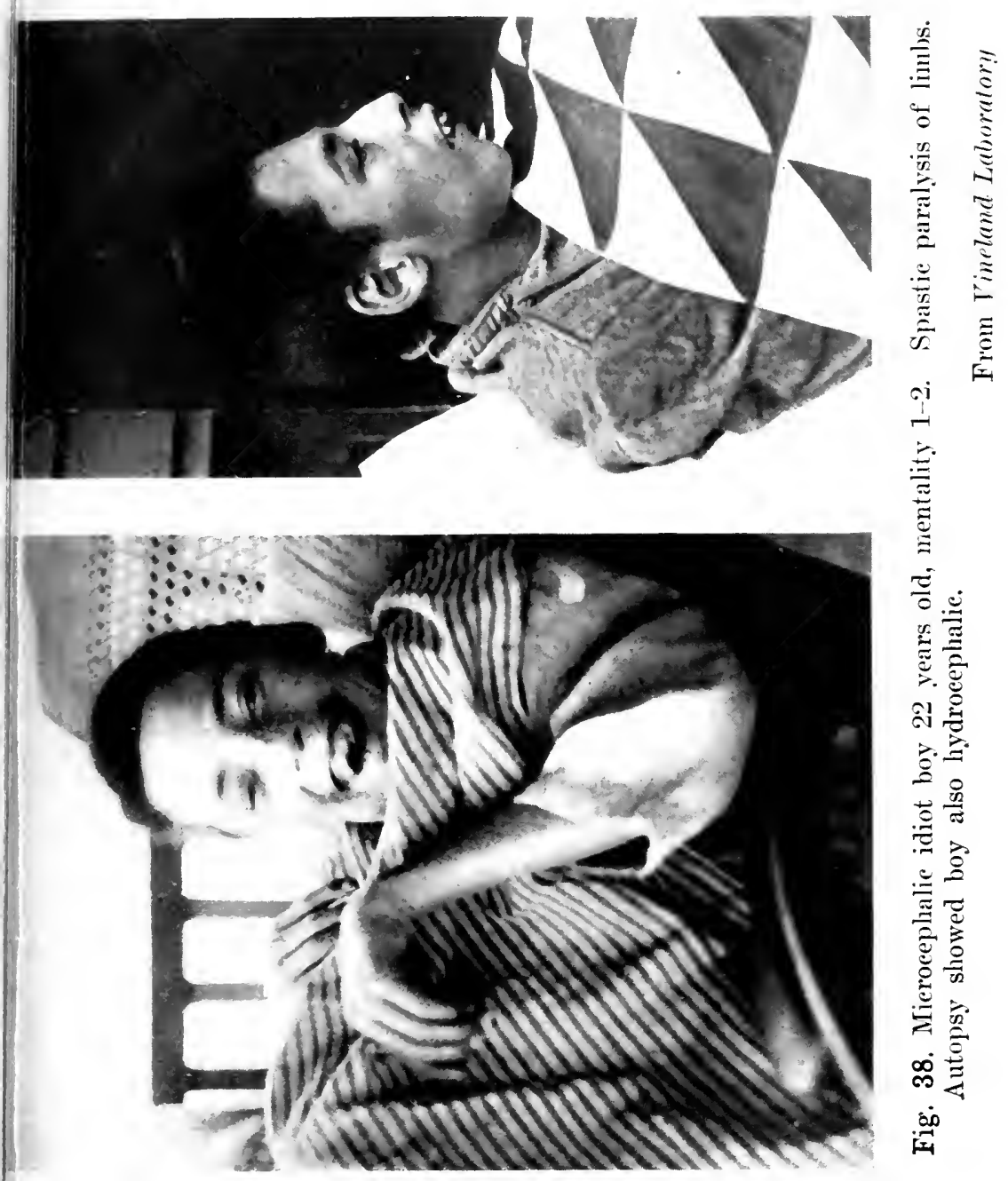




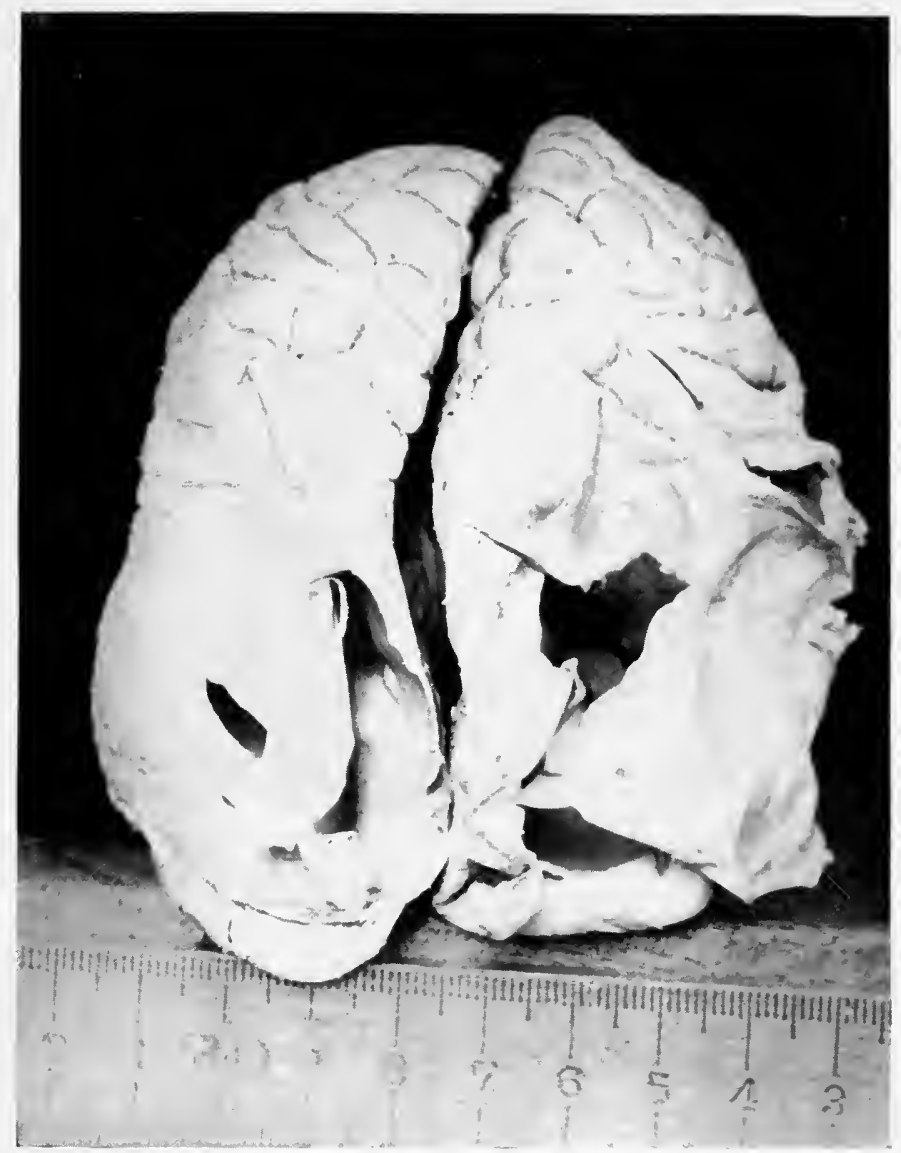

Fig. 39. Brain of ease pictured in Fig. 38. The occipital cortex is little more than a membrane. Microscopic examination showed that the cells had never developed. 


\section{ATTENTION OF DEFECTIVES}

child. Inasmuch as the idiot often has limited brain areas it is again evident that instinctive attention is compatible with very simple brain mechanism.

The accompanying picture, Fig. 38, is that of an idiot twenty-two years old, mentality 1 to 2 . Fig. 39 represents the brain of the same case. It will be noted that the cortex of the occipital region is almost entirely lacking. The child was microcephalic and also hydrocephalic, yet so "normal," for his grade, were his reactions that no one ever suspected such a condition. According to accepted views of brain localizaton he could have had only the slightest if any consciousness of vision; yet he smiled when one approached his chair as well as when spoken to. He seemed to respond to the smell of food and gave other indications of instinctive attention.

It is easy to understand, once this view has been mastered, that we do not focus our attention upon any one thing; but rather, when a stimulus is applied, a certain group of neurons are set into activity and consciousness results. For example, no matter what one is doing a loud noise will instantly "attract attention." We shall more accurately describe what happens, if we say there is a ready formed, instinctive pattern to which the stimulus of a loud noise instantly leads, arousing strong activity with its accompanying consciousness. But what becomes of the consciousness existent when the loud noise interrupted us? The answer is, it is eclipsed by the greater consciousness aroused by the new stimulus, and this principle must be borne in mind thruout: the greater eclipses the less.

Of two neuron patterns, each in activity, that one which is more active, which is developing the greater amount of energy will monopolize consciousness. That 


\section{PSYCHOLOGY OF THE NORMAL AND SUBNORMAL}

is the reason that as a rule the sudden loud noise holds our attention in spite of everything else. And yet there are circumstances under which one's consciousness is so intense that even a fairly loud noise is not heard. This only means that the neuron pattern, that was at the instant active, is stronger than that which is set into activity by the stimulus of the loud sound. Nevertheless there may be activity in the second area as is evidenced by the fact that while there is no consciousness accompanying it, nevertheless there is action. An observer might report that the loud noise produced a tremor, start, or even a jump in the individual who declared that he had not heard the noise.

Indeed we may go further; it is a common experience for a person to declare that he has not heard a question asked when in fact he had actually replied to it. In that case it is clear that the neuron pattern corresponding to the question asked was in activity, and even led to a response and the formation of words in reply; and yet because some other centre was more active and was producing a greater consciousness there was no consciousness connected with the first: or, one probably should say, such slight consciousness that it did not occupy the centre of the field and this explains why we are often able to utilize the experience without having been really conscious of the stimulus.

Another common experience is like the following: One is asked what time it is, "A little past three," "How da you know, did you hear the clock strike?" "No, I did not hear the clock strike, but yes, I believe it did strike." The explanation of this would seem to be that what the individual was doing at the time so engrossed his attention, that he was not attentive to the clock. Expressed 


\section{DIVIDED ATTENTION}

in our terminology: here are two neuron patterns in activity; the one corresponding to the particular thing that he was doing at the time was so much more strongly in activity that the striking clock was not able to arouse vivid consciousness. And yet there was some little consciousness, as is evidenced by the fact that later he is able to say, "Yes, I believe I did hear the clock strike." Again the strength of the conviction that he heard the clock strike varies all the way from almost nothing to a high degree. This may be explained by the relative strength of the activities in the two centres. If they are nearly equal then he is able to say, "Yes, I heard the clock strike, altho I was occupied with something else." If the other stimulus is exceptionally strong so that relatively the consciousness aroused by the striking clock is weak, he is in very great doubt and may even declare that the clock has not struck.

Similarly a great many common experiences are explained. Who has not had the experience of trying to recall a name, and being constantly baffled by the intrusion of a name which he knows is not correct. The results of what is called free association abundantly demonstrate the action of this principle, and are easily explained in this way. For example, let the reader get some one to pronounce a list of words to him with the understanding that he is to respond to each word with the first word that comes into his mind; and after each response let him stop and ask himself how he happened to make that response, how he came to think of that particular word. In every case he will find that the word has come to him in such a way that it is easy to see that the nerve energy aroused by the stimulus word has run into its own neuron pattern and given rise to a consciousness 
which was expressed by the word with which he responded.

The chief objection to the foregoing view is the insistent feeling that we do somehow direct our attention; whereas the above view makes us helpless in the matter, and we become the victims of pure chance, or better, of our previous experience. Many an intelligent reader will be inclined to say, "I know that isn't true. My own experience contradicts it." And yet, it is quite possible that our first or "common sense" view of the situation is a mistaken one; that we are under a delusion. It is not the only case, either in the realm of psychology or elsewhere, that we think certain results are due to our own activity when as a matter of fact our activity has nothing whatever to do with them. Let us take the instance last cited, of trying to think of a word. Do we not direct our attention first to one word and then to another until we finally get hold of the right one? At first thot it seems so, but let us see how we would explain this on the other theory.

According to the view here presented, the stimulus is the person, possibly in the form of a mental picture, whose name we wish to find. At once comes to mind the name Joseph but we know that "Joseph" is not correct, then comes James, and John and Judith and Jeremiah, we say we have thought of each one of these and discarded them in turn. What actually happened was, the stimulus aroused the neuron pattern which spelled "Joseph," but the circuit could not be completed. Then the nerve "energy flowed into another group of neurons which spelled "James," and then "John," and so on thru the list. "We" were doing nothing, the nerve energy was doing it all. Finally, the nerve energy flowed 


\section{ASSOCIATION}

into the neuron pattern which spelled "Justus," and that pattern was worked out because it was asociated with. the individual's face or his activity or with other experiences with him, so that the whole picture was consistent.

Consciousness has simply told us what has happened but without telling us how it happened; and we have made the mistake of thinking that the consciousness itself was the cause of the whole procedure. We shall have occasion to explain and discuss this principle more fully later on.

Another mental process inherent in the nature of the nervous mechanism is association. We have shown in a previous chapter how stimuli become associated. It is only necessary to refer to the subject here as another example of a property inherent in the nature of the neuron mechanism. Because the neuron patterns are what they are, two stimuli of about the same intensity, occurring at almost the same time, become connected in the brain and consequently connected in consciousness, and we say they are associated. If the stimuli are not of approximately the same intensity, the one predominates to the exclusion of the other and that one alone holds the field of consciousness or attention.

Since one or both of these neuron patterns may be stimulated indirectly from another centre of the brain, and such indirect stimulation results in the consciousness that has been given the name idea, as distinguished from consciousness aroused directly by an external stimulus which is called perception, it is common to speak of the association of ideas.

We have already shown that when two stimuli act at the same time each setting into activity its own brain pattern, those brain patterns become connected or associ- 
ated, and therefore the two consciousnesses aroused by the two stimuli are associated, as when I hear a sound emanating from an object which I see, the sound is associated with the object; and hereafter if I hear the sound I think of the object, or as I see the object I think of the sound. This process is of fundamental importance. Indeed this possibility of the association of the different mental patterns, the yoking them up together thru experience, underlies the whole mental life of an individual. Because of its importance it becomes desirable to discuss this process further.

The case cited is an illustration of what is commonly called association by contiguity, that is, the two stimuli are contiguous (literally touching). I see the object and, at the same time, hear the sound that it produces. Without this fact of contiguity, no association could take place, since each stimulus would arouse its own neuron pattern independently and lead to its specific action; or the energy would be dissipated and no connection would occur in the mind of the person. Life is full of such unassociated experiences. For example: In my daily walk to business I frequently meet a certain gentleman. He becomes a familiar sight to me, but here the experience ends. It also happens that in conversation with various friends they speak of a certain Mr. X and I become thoroly familiar with the name of $X$; but I may never connect the name of $\mathrm{X}$ with the gentleman that I meet on the street, unless in some way the two experiences of seeing the man and hearing his name come at the same time. This may come about as follows. While walking along the street I meet the gentleman and some one with me says, "That is Mr. X." Now the association is made and ever after when I meet him, I will think “Mr. X," when 
my friends speak of Mr. X, I will have a visual image of the gentleman as I have met him on the street. The two neuron patterns in my brain have become thoroly connected. This is the simplest and most direct way of making the connection. But it may be made in many other ways. From a neuron pattern standpoint the matter is always the same; the two neuron patterns are excited.

It matters not whether the pattern is actually arousul by the external stimulus or whether it is aroused indirectly from some other centre of the brain. That is to say, instead of actually seeing the gentleman, his imagre may be called up and that image connected with the name. As for example, some one says to me, "You know Mr. X." I say, "Yes, by reputation, I have often heard him spoken of." To which it may be replied, "But you know him, you meet him every day; he is that tall, broad-shouldered man that you meet as you go to business." The description of a broad-shouldered man calls up the mental image of the gentleman, and now my friend says, "That is Mr. X."

It will be seen that this type of occurrence is constantly happening; we thus learn the names of people and objects, and associate various qualities and characteristics of objects. If one arouses a neuron pattern by the word iron, I immediately think "heavy," and it is easy to see that I have at some time lifted or attempted to lift something that I knew was called iron. I got certain sensations or had a certain neuron pattern aroused which meant muscular exertion of a high degree, and which gives the consciousness "heavy." Without the experience it would be impossible to think "heavy." It would not be impossible for me to say "heavy" because the association iron - heavy may have been made for me in a purely verbal 
way. I may have been told that iron is heavy, consequently when some one says iron, I say heavy. But it will be recognized that $I$ do not have the same mental content that I had when I actually lifted iron. If I know what the word heavy means from the actual lifting of other heary objects, I may be able to think of the iron as heary with a considerable degree of accuracy. Nevertheless, my knowledge that iron is heavy is not quite the same and not quite so satisfactory as tho I had actually had the experience of lifting iron. Nevertheless, much of the experience that enters into our mental life is of this ricarious kind. For example, I am quite satisfied that common white arsenic is sweet. I have never tasted white arsenic, but I have tasted things that are sweet, and I know what is meant by sweet; consequently when I am told that arsenic is sweet I have the ability to think of arsenic in much the same way as I would if I had actually tasted it. Nevertheless, it will be recognized that it is not exactly the same and should I taste it I would probably find that I knew arsenic in quite a different way from what I had known it before.

Some writers have distinguished these two experiences as knowledge of the "first order" and knowledge of the "second order." If I have actually seen Barnard's statue of Lincoln I have knowledge of that statue of the first order, but if I am told that there is a statue of Lincoln by Barnard, that it represents him standing, and the peculiarities of that statue are described more or less in detail, I have a knowledge of the statue of Lincoln, but it is knowledge of the second order.

In our illustrations of the memory of the feeble-minded, the reader will see that much of it involves simple association. The name of a person, of course, is an associa- 


\section{ASSOCIATION IN DEFECTIVES}

tion between the visual perception of the face and the auditory image of the name. The lower grade defectives make only a few associations of the simplest character and practically always by contiguity and as the result of a greater or less number of identical experiences. Even the highest grade defectives associate largely by contiguity, the difference between them and the lower cases being that they make more associations and form them more quickly; that is, as a result of fewer experiences, in many instances apparently forming them as quickly and easily as normal people.

Florence was being given one of the Norsworthy Tests (Genus-Species Test) where, given the name of the genus, the child is required to name a species; e.g., plant -rose. When the test was about half done the examiner remembered that Florence had had the test the previous year, whereupon he asked her if she remembered having the test the year before. "Yes," she replied, "but I'm not giving you the same answers that I did a year ago." "What was your answer to this question at that time?" Her reply was recorded, and when the records of the previous examination were looked up, was found to be correct. She not only remembered having had this list, but remembered her exact answers. It is evident from such experiences that the simple association fibres connecting the different centres are sufficiently developed, especially in the feeble-minded of higher grade, to enable them to make a great many associations by contiguity.

We have now seen how some of what are called the simpler mental processes are the direct outcome of the nature and structure of the nervous system. There is still another manifestation of mind which, tho somewhat more complicated, seems also to be inherent in the nature 
of the nervous mechanism and therefore belongs in this group. This is emotion. But its concideration will be postponed until we have discussed the elaboration brot about in the foregoing processes by the development of the great association centres.

\section{Summary.}

1. Because nervous substance is the most highly organized matter known, it is most highly irritable, a stimulus produces not only temporary but permanent change. This is the basis of memory, natural retentiveness.

2. The mark of memory is a feeling of familiarity, due apparently to the greater ease with which the neurokyme flows thru a pattern already in use.

3. If the pattern is unused for a time this feeling of familiarity may be lost. One may then reproduce the image without being conscious that it has been experienced before. This is reproductive imagination.

4. Both inherent memory and reproductive imagination are possible in the feeble-minded because they involve relatively simple neuron patterns.

5. Because nerve substance is what it is we have memory and imagination; because it is arranged as it is (neurons, synapses, etc.) we have attention and association.

6. Attention is the most vivid consciousness. This vividness depends upon strength of stimulus, nature of neuron pattern (inherited or acquired), elaborateness of neuron pattern (simple or complicated), and upon frequency, recency and emotional content.

7. Two or more centres of the brain, being stimulated simultaneously or nearly so, become connected by as- 


\section{SUMMARY}

sociation neurons, and therefore the stimuli arousing these centres are associated in mind. Thereafter the activity of either one will set into activity the other thru association by contiguity.

8. This association process thru the functioning of great areas of association neurons becomes the condition for a great extension of all the mental processes.

9. Both inherent attention and association by contiguity are within the capacity of the feeble-minded. 


\section{CHAPTER V}

\section{HIGHER MENTAL PROCESSES}

WE have now completed our survey of the simpler mental processes. While some of these may involve rather complex neuron patterns, they are nevertheless connected with and depend upon the more primitive parts of the brain. These parts are very early developed and many of the neuron patterns are inherited, ready made, while the rest are quickly formed by the simple experiences of every day life.

This agrees thoroly with the obvious fact that very young children and animals, even fairly well down the scale, have all these mental processes. That is to say, they remember, they attend, and they associate. That these are phylogenetically the older, more primitive portions of the nervous system is also attested by the fact that many animals have the processes of memory, attention, etc., who have what are relatively very elemental nervous systems-very small and simple cortical areas and only a fraction of the convolutions that are found in human beings.

All this marks what may be considered from one point of view a rather sharp break between these more elemental manifestations of mind and the more elaborate development of the higher processes of thot, reasoning, judgment and will. While from a broad consideration it is perfectly true that there is no radical break but rather a gradual development into those higher processes, yet 


\section{HIGHER PROCESSES}

we shall see later that a marked absence of them characterizes various stages of immature mind.

We must now proceed to trace as fully as our knowledge will permit the development of these higher processes. The logical place to look for this development is in the extension of the association system. For the processes already described we have needed only the comparatively simple neuron patterns located in any one centre or in two or more of the localized centres with simple association fibres connecting them. We repeat the observation made earlier that the completion of the process started by any stimulus involves muscular activity; and as long as the force of the neurokyme is relatively unimpeded from the stimulation of the first sensory neuron thru the various synapses over to the motor neuron with the resulting muscular activity, no other pathway is needed and none other will be followed. It is generally held that we should look for the condition which leads to the extension of this system in some kind of blocking of this simple path of the nerve energy. This may readily be conceived as coming about from the interference of two or more stimuli. In the complexities of the environment of the higher animals and especially of man, a familiar stimulus may have for its natural result an action which is antagonistic to some other instinctive tendency, for example, instinctive curiosity leads a child to explore a new object, but the object, possibly a dog, emits a sound which arouses the primitive emotion of fear. The natural outcome would be movement away from the object rather than toward it. One has often seen this conflict going on in young: children as well as in animals. Curiosity leading them to approach, fear leading them away. The conflict be- 
tween the two results in no movement whatever, and a common description of the situation is more or less correct; we are very apt to say, "He doesn't know what to do and is trying to decide, he is thinking about it."

Going back to our picture of the neuron patterns we may say that the course of the neurokyme is blocked; not being able to flow into its usual channels, it flows in a new direction; and we may for the moment omit a number of difficult intermediate steps and say that the neurokyme has flowed into the great association centres, and we have therefore developed more elaborate patterns than ever before; some of the higher mental processes such as thot, reason and judgment are the result.

We cannot, however, leave the matter just here, but must make an attempt to picture to ourselves how it may be possible for this development to take place. We have already explained (p. 84) association by contiguity; that is, when two stimuli $\mathrm{A}$ and $\mathrm{B}$ are presented at the same time, there is a tendency for the nerve energy aroused by each stimulus to pass back and forth over the path connecting the two centres $A$ and $B$, so that when on a later occasion stimulus $A$ is presented, the centre originally aroused by the stimulus $B$ will be again aroused, but this time indirectly from centre A. It must be emphasized that the contiguity is a contiguity of the two stimuli; that is of the situations or experiences which give rise to the stimuli; so that in reality it is contiguity of experiences that gives rise to the association.

One has only to review his experiences to see how large a part in life this kind of association plays. One sees two people together; if upon a second occasion he meets one of those persons he is very apt to think also of the other one. One visits a new place in winter when there 
is snow on the ground; he may visit the same place the next summer, and will be apt to think of the snow that was on the ground on the former occasion. One may meet with an accident during which many things happen; upon a later occasion the reappearance of any one of the objects or persons is apt to recall more or less completely the other persons or objects or circumstances involved in the original experience; and so on ad libitum.

Now let us turn to another kind of experience. While walking with a friend you meet a stranger. After pass. ing you say to your friend, "Of whom does that person remind you?" the friend says, "Of no one." "Does not his walk remind you of X?" "Why, yes, he does walk like X." In your mind that person was instantly associated with $X$. In the mind of your friend there was no such association. Why the difference? In the first place, why did you associate him with $\mathrm{X}$ ? Your question gives the answer. It was his walk. You are well acquainted with $X$, you have seen him many times, and more or less unconsciously his walk has made an impression upon you, there has been an association of contiguity between the walk and $X$-simply because it is $X$ 's walk. You cannot see him without seeing his walk. Now you see that same walk in some one else, but it suggests $X$, because there is a connection in your neuron patterns. That walk is tied up, so to speak, with $X$. The neurokyme flows into the nerve pattern that has been so many times aroused by seeing $X$ walk.

But your first question was not, "Of whom does that walk make you think?" that is, with whom is that walk associated, but, "Of whom does that person make you think?" with whom is that person associated in your mind; and your friend had no association with any per- 
son. It is clear that in your own case the one thing that you noted about the stranger was his walk and, as already stated, that walk was by past experience associated in your mind with $\mathrm{X}$. On the present occasion it is associated with the stranger. We therefore find that by the medium of the walk the stranger is associated with $\mathrm{X}$. This is called association by similarity. The stranger is similar to $\mathrm{X}$. The similarity is not marked, in fact it is very slight; it pertains only to his manner of walking, a minute detail which had never entered into consciousness with your friend. The friend did not see any similarity between the two people because he did not .become especially conscious of the walk. When you brot it into his consciousness by your question, then it was evident that there was an association by contiguity in his mind between $X$ and X's walk and that he also had noticed the stranger's walk and he was able to say, "Yes, he does walk like him."

This is the essence of all association by similarity. Two things are similar when they have some point of identity. When we associate by similarity we are always able to pick out the identical points; and if we do not associate two things that are similar it is because the point in which they are identical does not come into our consciousness. Your friend was perhaps looking at the eyes, or at the clothing, or at some other feature rather than the walk, hence there was nothing to arouse the association by similarity which, as has often been pointed out, is in reality only a special form of association by contiguity. But it is convenient to separate the two and consequently we shall follow the usual custom. It is a common experience to say, "That person makes me think of so and so"; "Your house reminds me of the one I 


\section{SIMILARITY}

lived in years ago"; "That tree makes me think of the elms that grew on the home place." When we remember that life is experience, and ask ourselves why the one thing reminds us of the other-becomes associated with it-we are apt to say they are similar, hence the expression association by similarity. But just what is similarity and how can we picture what happens in terms of neuron patterns?

Always, when one thing suggests another or when we say two things are similar, it will be found that there is some point in which they are exactly alike. The fact that the one reminds us of the other, or that we notice the similarity, proves that the particular feature is the one that has aroused our attention, or in other words, set into activity a particular brain pattern. That particular brain pattern is now associated (by contiguity) with other brain patterns which together make up our consciousness of the whole object. All the different features of the object are present to us at the moment; but the particular brain pattern involved in the similarity has in time past been a part of another picture also associated by contiguity.

Our association by similarity then is simply the connection of a present elaborate neuron pattern with a past elaborate neuron pattern by means of one part of each pattern that is common to both. There is thus nothing new involved. It is simply the matter of contiguity which, as we have stated, is the basis of all association. Nevertheless, it is convenient to separate this group from the other because of the somewhat round-about way in which the association occurs. It will be seen at once how this concept enlarges our appreciation of the rôle that association plays in the building up of mental life. 
We shall see later that logical memory is entirely a matter of association, and we shall see still further why memory becomes as elaborate as it does, and how it comes about that we are able to recall so many different things that would seem at first utterly impossible to remember. For example, upon thinking of Harvard I think of Yale, not because they are contiguous or have ever been together in my experience, but they are both universities and Yale University by way of the concept university at once suggests Harvard University.

It should be noted that here, as in many other cases, there may be a direct association by contiguity by way of words, what we may call a verbal contiguity. While I have never seen Harvard University and Yale University at the same instant, I have seen or heard the two words, Harvard and Yale, at the same time. So that it is not always possible to say definitely whether the association is of the pure contiguity type or of the similarity type, unless one knows at the same time what we might call the natural history of the experience. Again it may be pointed out that even verbal associations may be by similarity as well as by contiguity. This is the basis of all punning, where we have an association by similarity of sound.

It is thus seen again that in the last analysis the capacity of making associations depends upon two factors. First, experience; and second, a neuron system that is able to respond to the different stimili. Either factor being absent, no association is possible. Either factor being weak, the association is weak and incomplete, unsatisfactory and inefficient.

Now, undeveloped mind, either of the child or of the mental defective, in proportion to the incompleteness of 


\section{ASSOCIATION IN CHILDREN}

its development, is incapable of elaborate associations. The child under about seven years of age may be incapable of elaborate associations because of lack of brain development; but if, as is generally supposed, the brain has attained nearly its complete development by the age of seven, thereafter any inability to make elaborate associations will be due to lack of adequate experience. It is probable, however, that the brain normally develops much beyond the seven year period, because the phenomenon of mental defectiveness shows us that people who are adult in years and have had all the necessary experiences are nevertheless not able to make the elaborate associations that underlie thot, reasoning and judgment. Their experience is adequate but there is somewhere an incapacity in the brain system, consequently the desired results do not follow.

From this it is possible to make two very fundamental and important deductions. The one is, that the capacity of the individual for higher associations and elaborate thot is absolutely limited by the state of his mental development, and that all attempts to develop those higher processes are and must ever be futile. Conversely, when experience has shown us that it is impossible to teach a child those things which children of his age ought to learn, we may be satisfied that there are in that brain defective neurons; and it is as impossible to develop those defective neurons as to make a successful athlete out of a cripple. These considerations give the answor to the question, "Why is be so stupid, why can he not learn?"

We have described in an earlier chapter what is now accepted in brain localization, that is, the parts of the brain that are known to be essential to certain functions. 
The remaining areas of the brain, the great frontal region, the region between the kinesthetic and the optical centres, and the large area between the optical and the auditory centres have been designated by Flechsig as association areas. It is thot that in these areas are found the neurons that are involved in the higher and more elaborate associations.

Herrick (23, p. 290) says: "It must be borne in mind that the most significant parts of the human cerebral cortex are the association centres. These alone are greatly enlarged in the human brain as compared with those of the higher apes. In the latter animals the projection centres are fully as large as those of man, the much smaller brain weight being chiefly due to the relatively poor development of the association centres."

And on page 294: "It is a fact of common observation that those animals which possess the capacity for intelligent adjustments of this sort have larger association centres in the cerebral cortex than do other species whose behaviour is controlled by more simple reflex and instinctive factors, that is, by inherited as contrasted with individually acquired organization. This is brot out with especial distinctness by a comparison of the brains of the higher apes with that of man, and of the lower races of men as contrasted with the higher."

It seems logical to conclude that, in the case of mental levels below the normal, these centres must be considered lacking in development. We may picture more or less of the cells in these regions as like the smaller ones in Fig. 13, whereas they should be like the larger ones. The exact mechanism, the way in which these centres operate in the elaborate associations of the intelligent man, is of course far beyond our knowledge at the 


\section{VALUE OF ASSOCIATION BY SIMILARITY}

present time. We do not even have the evidence that these centres are undeveloped in the defective, but it is believed by our best neurologists that when our technique is able to grapple with this problem it will clearly show a defect in these regions.

Again one has only to recall his experiences to realize that this association by similarity plays a very large part in one's life and it plays not only a large part but a vitally important one. In fact it is the fundamental step in the larger development of mind. If we were limited to "association by contiguity" a large proportion of our experiences would be meaningless to us and consequently useless. We should never know how to act in a new situation. One thing would never suggest another to us unless the two actually had been presented to us previously in contiguity. The law of gravitation would never have been discovered because it required the perception of the similarity of the apple falling to the earth and the earth falling to the sun. Inventions would be impossible because the inventor must see the similarity between something that is already experienced by him and the new thing that he wishes to accomplish. The burnt child would not dread the fire unless it was precisely the same kind of fire as burned him the first time. He would not see the similarity between the red hot blaze and the red hot piece of iron.

There are all degrees of similarity. In our first example of the stranger's walk we said that the similarity between the stranger and $\mathrm{X}$ was not marked. It was very slight. It is clear that the more marked the similarity the more easily association is made; so that when we say that without the association by similarity we would be deprived of all these possibilities, we are 


\section{PSYCHOLOGY OF THE NORMAL AND SUBNORMAL}

speaking in general terms and with only a partial view, merely for the sake of illustration and simplicity.

As a matter of fact it is unthinkable that we should not reason by similarity to a certain extent if we associate by contiguity; because many times nothing about the new object arouses our consciousness except the phase that we have experienced in some other connection. In other words, association by similarity passes imperceptibly into association by contiguity. Two houses are so nearly alike as to be practically identical as houses. The one suggests the other strictly by similarity because they are not the same house; and yet because they look so much alike it is practically a matter of contiguity. Even undeveloped mind may associate by similarity in such instances. We shall understand later that it is in a sense a measure of intelligence to perceive similarities of the more difficult types.

All this is clearly understood again by reference to our neuron patterns. That is to say, it depends upon how elaborate has been the neuron pattern aroused by the earlier experience or experiences of contiguity. My neuron pattern of $\mathrm{X}$ had been elaborated to include his walk. My friend's neuron pattern of $\mathrm{X}$ had not so definitely included that feature; hence the difference between us. With me the stimulus of the walk aroused the entire $\mathrm{X}$ pattern; with my friend, the stimulus of the walk aroused no such pattern, either because it never had aroused the pattern or because the walk part had been so light, so vague, so indefinite, that it did not arouse enough energy to set going the whole pattern. Or it might be that my friend's neurons are not sufficiently developed so that he could have as elaborate a pattern as I have. 


\section{LIKENESSES VERSUS DIFFERENCES}

This brings us again to our fundamental principle of brain and neuron development. The person whose neurons have not developed properly cannot possibly have those more elaborate patterns. Consequently we are safe in concluding that they never can associate by similarity to the fine extent that other persons can whose neurons are better developed. There seem to be people in whom it is impossible to develop any artistic sense. We may readily conclude that their neurons are not sufficiently elaborate for them to have these more extensive patterns which enable them to perceive those similarities which go to make up artistic appreciation. Furthermore it seems clear from what we know of brain development and the age at which these finer associations are possible, that these elaborate neuron patterns require an extension into the association areas of which we have spoken.

When we study the actual performance of various stages of undeveloped mind, we find evidences of very definite development of the association process. Speaking in broad, general terms we can state that the feebleminded associate by contiguity but not by similarity. Children do not easily see similarities. Differences are perceived before similarities. Ask a child of seven in what way coal is like a stone and he will tell you we can burn coal and we can't burn a stone. It is the difference that he notices. Not until eight years will he give the correct answer. And not until eleven will he see likenesses as often as differences (see p. 294). This agrees well with what we have already pointed out that association by similarity involves more elaborate neuron patterns than association by contiguity.

This helps to establish the view already hinted at that [101] 
the neurons lacking complete development in the feebleminded are those that are located in the great association centres; and further that since the upper limit of feeble-mindedness is somewhere around twelve years, or at the beginning of puberty, it would follow that the great association areas get their complete development after that period. Of the whole brain, these neurons are the latest to develop and consequently most likely to be affected by anything that causes arrest of growth.

When one realizes to what extent the ability to adapt one's self to environment, to meet the various situations that arise in the course of the day, is dependent upon association by similarity, one easily understands why the feeble-minded are incapable of managing their own affairs with ordinary prudence; and one understands the otherwise puzzling experiences and peculiar limitations of the feeble-minded. A common way of distinguishing the feeble-minded from the normal is to say that they cannot adapt themselves to changed conditions. The higher grades of feeble-minded can be trained to do quite elaborate tasks and seemingly ought to be capable and efficient workers, but it is just this limitation that makes them fall short of efficiency. There are relatively few situations in life that do not require some variation from previous experience.

In other words, the normal individual solves the new problem because of its similarity to a previous problem. The feeble-minded can solve a problem if he has been trained to solve exactly the same kind of problem on a previous occasion. Let us illustrate: We have devised a test for this, a piece of apparatus which we call the Adaptation Board (20). It consists of a piece of half inch wood $22 \times 28 \mathrm{~cm}$. Thru this are bored four holes. Three 
of the holes have a diameter of $63 \mathrm{~mm}$; the fourth has a diameter of $65 \mathrm{~mm}$. The whole is finished (painted, or stained), so there is no obvious difference between the two sides. A circular block is prepared, preferably about an inch thick, and $64 \mathrm{~mm}$, in diameter, so that it will fit easily into the larger hole, but will not go into any of the other three.

The method of procedure for the use of the test is as follows: The child to be tested stands at the left side of the examiner; the board is in front of them on a table, in such a position that the hole into which the block will fit is in the upper left-hand corner. Directions are given as follows:

Examiner: You see this block, and you see these holes. The block will fit into only one hole. Find which one.

By trial the child discovers the correct hole in the upper left-hand corner and places the block in. The block is removed by the examiner, who says, "Do it again. Once more place the block into the only hole it will fit." This may be repeated until the child places the block, without hesitaton and without trying in any other place, in the correct hole.

Examiner: Now you take the block. Now watch me carefully.

The examiner turns the board over from left to right, slowly, taking about 2 or 3 seconds for the turn; then turns to the child, "Now put the block into the only hole that it will fit." Those of sufficient intelligence will place the block at once into the upper right-hand hole. Another group, however, attempt to place the block still in the upper left-hand hole, not having been able to adapt themselves in the slighest degree to the changed condition. Failing to get the block into this hole, the child 
tries until he discovers that it goes into the upper righthand hole.

The examiner now places the board back in its original position, with the large hole in the upper left-hand corner. Proceeding as before, he shows the child that the block goes in the upper left-hand hole. This being learned, he again says, "Now watch me carefully," and turns the board at the same rate as before, but instead of turning the board from left to right, he turns it from back to front, that is the side farthest away from him to the position nearest, and says, "Now place the block in the only hole into which it will fit." We now have three groups : first, those who succeed promptly; second, those who fail entirely, still trying to block in the upper lefthand hole; and third, an intermediate group, who try the block in the upper right-hand hole. Apparently, these last are children who are not able to adapt themselves perfectly to the changed condition; but who do remember that, after the board was turned the first time, the block went into the upper right-hand corner. Not having been able to distinguish the difference in the directions in which the board was turned, they again try the block in that position. The accompanying chart Fig. 40 shows how the ability to adapt to the changed position of the board increases with the mental age of the child. It is seen that almost $90 \%$ of defectives with a mentality of eight or above, having learned which hole the block will fit into, are able to fit that block into the hole when they have seen the board turned over, but under eight the percentage able to adapt themselves to the changed position of the board grows steadily less.

Success with the adaptation board obviously requires a very simple adaptation. Consider a somewhat more [104] 


\section{ADAPNATIOH BOARD}

EERCENTAGR OP SUCOESSES OH SECOWD FUR.

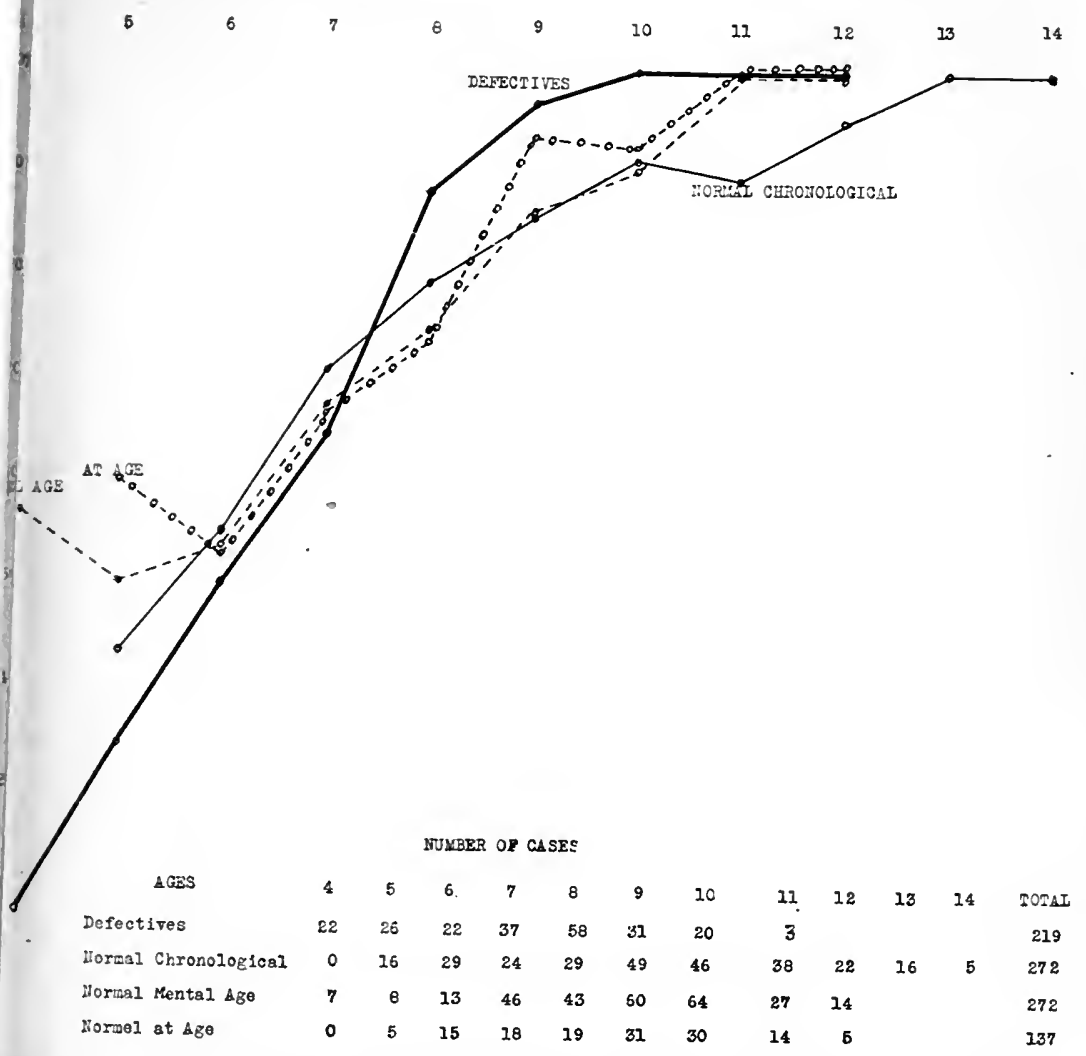

Fig. 40. Curves showing development of ability as measured by the Adaptation Board 



\section{ADAPTATION BOARD}

difficult adaptation. A girl with a mentality of 11 had been trained to make beds; she could by herself make a bed almost perfectly. One day a normal adult offered to assist her in making the bed. The girl was totally unable to do her part. She could make the bed alone but was unable to adapt herself to the changed condition of having some one else assist in smoothing out the bed clothes. It was a new problem to her and she was unable to see the similarity and adapt herself to it. A person of this mental development, trained carefully and patiently to do the work in a certain kitchen, is utterly confused and helpless if placed in a kitchen where the arrangement of apparatus is different from the one to which she is accustomed. The kitchens are closely similar but not the same, and she is incapable of perceiving the similarity and adapting herself to the changed conditions.

Classification is a process that involves perceiving the similarities between things, and arranging them according to some principle of similarity. The feeble-minded are incapable of making any useful classifications except those of the most extreme simplicity. If children under twelve years of age are asked to name sixty objects it is found that only the older ones are able to associate one name with another by similarity or according to any classification or common grouping. If a child under nine names "pen" he will not think of "pencil" or "paper" or "ink" or any of the objects that an adult would naturally associate with the pen. If the child is of rather low grade we find him absolutely limited to the things that he can see. He looks around the room and names the objects in sight and then stops. The objects that he sees do not even suggest to him similar objects that he has 
seen elsewhere or on other occasions. The neuron patterns that are involved in making these associations by similarity have never developed in his brain and consequently he is incapable of thinking things out that way. We need not dwell further on this at the present time, for we shall see the same thing in a closely allied manifestation of mind, namely attention.

Attention.-We have described what we call instinctive attention as the consciousness that is aroused by any stimulus that sets into activity an inherited nerve pat. tern. But just as we have seen that there is an exten. sion of the simple association, so there is an extension of the attention. We find ourselves attending to many situations that certainly make no instinctive appeal. That is to say, they cannot by any possibility be considered as belonging to any inherited neuron pattern. For example, in a drawing room where many people are talking in groups I am talking with a friend. Suddenly I catch the word "psychology" uttered by somebody in a group near-by. Instantly my attention is held or attracted, so much so that I entirely miss the remark that my friend is making and have to call myself back and beg his pardon and ask him to repeat. What happened is this: In the midst of my consciousness of my friend's conversation, the stimulus of the word psychology suddenly aroused a neuron pattern which swamped all the other patterns that were active in my brain.

Why did that word psychology change my thinking or my consciousness? Certainly not because I was born with a neuron pattern ready made to respond to the sound of the word psychology. That is an acquired pattern. It is true that if we could trace the etiology of that new experience we would find it ran back to something instinct- 


\section{ACQUIRED ATTENTION}

ive, but we need not here attempt to trace that course be cause we have already done so under the head of association. This bit of acquired attention comes thru the extended association. As the result of experience the word psychology has come to be associated with something that is instinctively significant for my life. It may be that I earn my living by teaching psychology and so it comes about that the fundamental instinct of self-preservation has become associated with this newly acquired technical subject of psychology. The original inherited neuron pattern has become elaborated to take in this matter of psychology, consequently when I now hear the word psychology it arouses a very large and important neuron pattern, the consciousness connected with which is so strong that it swamps all other matters for the time being.

Again we note that this capacity for attending to all these things that do not directly appeal to instinctive interest is a mark of higher development of mind and of neuron patterns. And again we may see that one may be lacking in this capacity either from never having had the experiences which would develop these patterns, or from not having neurons sufficiently developed to accomplish it. A person whose association centres had not developed would not be capable of making these acquisitions or of having what we call acquired attention. We must repeat the caution that we are speaking broadly. The transition is gradual from instinctive to acquired attention. Even children make many acquisitions but of course are not capable of the concentration we find in adults.

One of the complaints most often made of mental defectives is that they cannot concentrate their attention. Teachers and parents, not understanding the nature of 
the human mind, often say, "If we can only make this child concentrate his attention he will be all right," which is a perfectly true statement but meaningless. He cannot concentrate his attention because he has not the necessary neuron patterns. What is meant is, that the child has acquired few associations; he attends to those things that appeal to him instinctively. But those things that depend for the arousal of consciousness, which means attention, upon the combinations of elaborate neuron patterns based upon experience, he cannot do. He may have had the individual experiences but he has not woven them together in such a way that they make a complex picture because he does not have the association neurons.

This lack of acquired attention is characteristic of the feeble-minded. It is this that makes their conversation and their actions so childish. The brain being arrested in its growth before the neurons in the association areas have developed, the feeble-minded are not able to build up those elaborate neuron patterns which must underlie the elaborate consciousness that means attention to larger problems or interest in the greater affairs of life. The feeble-minded are thus characteristically lacking in all these higher mental processes. Their association by similarity is very limited, consequently associative memory is weak, acquired attention has limited range, and constructive imagination is largely lacking. Moreover, the capacity for each and all of these processes varies as the mental development. Of all classes of defectives, the moron has the most development of these processes, the imbeciles less, and the idiots least, indeed practically none.

The best explanation of all this, in view of what is [108] 


\section{SUMMARY}

known of the nervous system, is found in the view that these higher processes depend ppon the development of the association centres, and that these centres probably develop mainly after the beginning of adolescence. Consequently any arrest in that development previous to that time leaves the child without the necessary neurons for making these higher associations. And the earlier the arrest, the fewer the neurons. We see exactly the same condition of things in still another manifestation of mind; namely, memory.

\section{Summary}

1. The development of the great association areas marks a great advance in mental functions.

2. Animals, young children and mental defectives are differentiated from normal adults by the absence or weak development of these areas and the consequent marked deficiency in the higher mental processes.

3. Association by similarity is little manifested in immature mind. Ability to see obscure points of similarity is a mark of intelligence.

4. The ability to adapt one's self to new situations and new problems depends upon association by similarity.

5. Attention is greatly extended by the elaborate associations resulting from the perceptions of similarities. This is acquired attention.

6. Persons of incompletely developed minds cannot "concentrate their attention" because their association fibres are not developed.

7. The feeble-minded are characteristically deficient in, acquired attention and association by similarity. 


\section{CHAPTER VI}

\section{HIGHER MENTAL PROCESSES-Continued}

Memory.-We have described inherent memory, or what some have called natural retentiveness, a condition depending upon the nature of nerve matter and upon direct experience. But we remember many things that are not matters of direct experience. We might stop and consider what would be our condition if we were limited to the kind of memory already described. We could remember only the things that came to us thru the senses. The neurokyme would flow into these simple neuron patterns that had been aroused by the original experience and we would have the feeling of familiarity and remember that experience. Thru these acquired associations and the great extension of the nueron patterns into the association centres, we get a coridition which permits the nerve energy to run into this elaborate pattern, arousing the corresponding consciousness and arousing it with that greater ease which means the feeling of familiarity, the mark of memory. Memory thus becomes enormously elaborated and we speak of this elaborate form as associative or acquired memory, because it is dependent upon acquired association, association by similarity. This is also called logical association.

Herrick (23 p. 295) says: "In our own mental life we recognize the persistence of traces of previous experience subjectively as memory, and memory lies at the basis of all human, culture. From this it follows that psychological memory is probably a function of the association 


\section{ASSOCIATIVE MEMORY}

centres; but it must not be assumed that specific memories reside in particular cortical areas, much less that they are preserved as structural traces left in individual cortical cells, as has sometimes been done.

"The simplest concrete memory that can appear in consciousness is a very complex process, and probably involves the activity of an extensive system of association centres and tracts. That which persists in the cerebral cortex between the initial experience and the recollection of it is, therefore, in all probability a change in the interneuronic resistance such as to alter the physiological equilibrium of the component neurons of some particular associational system. What the nature of this change may be is unknown, but it is conceivable that it might take the form of a permanent modification of the synapses between the neurons which were functionally active during the initial experience such as to facilitate the active participation of the same neurons in the same physiological pattern during the reproduction."

The writer once met a patient in a hospital who, thru an injury to the head, had been made practically aphasic; he was unable to remember the names of common objects or of familiar persons and places. If one pointed to his coat and asked him what it was he could not answer. If he were told that it was a coat and then his vest was pointed to, he could say vest, and could name the other articles of clothing. But if one pointed to the pencil in his hand, he could not name it. When told that it was a. pencil he could then name the paper, the ink, etc. Asked where he lived, he could not tell, but after some little time, during which he seemed to be struggling hard to recall the address, he finally said " $1,2,3,4,5,6,7,8,9,10,11$, 12, 13, 1313 South Street," which was correct. When 


\section{PSYCHOLOGY OF THE NORMAL AND SUBNORMAL}

asked the name of his nurse he went thru the same struggle and finally said, "Sunday, Monday, Tuesday, Wednesday, Thursday, Friday, fish, Miss Fischer."

We have in these examples an illustration of memory and recall by means of a logical association. In the case of the coat or pencil, it was an association to the general concept of clothing or writing material and while he could not get it even this way at first, when once that general centre was aroused by his being told that the first object was a coat, it was then easy to arouse the neuron pattern that stood for vest or other articles of clothing; similarly with the writing material. When asked for the place where he lived he could not make the direct association between "where I live," and "1313 South Street," but he knew that he wanted a number and counting being a relatively automatic matter had not been destroyed by the disease, so that he was able to say $1,2,3$, and when he got to 13, was able to say 1313 South Street. The last illustration is perhaps the most interesting of all since it shows a very roundabout way of associating his nurse's name with fish, and fish with Friday of the week. Again, the names of the week being automatic he was able to get hold of them, and starting with the first to enumerate them until he got to the one he wanted. We see the situation clearly in this special instance, where the disease had destroyed the extraneous associations and left only the fundamental ones which have been used most.

But the plan, once understood from this special case, is easily discernible in many of our ordinary memories. Who, for instance, has not had the experience of going to another room for some desired object and arriving in the room, not being able to remember why he came. His method of recall will be exactly like that of the aphasic 
patient. He will recall what he was working on, what he was thinking about, how far he had gotten in his problem and what it was that he needed, and by this roundabout process it will finally result that the nerve pattern that underlies the name or appearance of the object that he wants will come to consciousness and the problem is solved.

One reads in the paper of Vladivostock and he asks himself what and where is Vladivostock. The probabilities are that his school geography association has long since been obliterated, but he is reading about Russia. He concludes that it is somewhere in Russia; the context indicates that it is a city, a city in Russia; he thinks of the cities of Russia ; he thinks how he would go by rail from Moscow to St. Petersburg, from St. Petersburg somewhere else; he thinks of the great Trans-Siberian Railway, and gradually and, at first vaguely, it comes to his mind that Vladivostock is the Pacific terminal of the Trans-Siberian Railway, and his question is answered.

We have already seen how names of objects are acquired by association; seeing the object and hearing it named at the same time results in a connection between two neuron patterns, so that whenever either part of the combined neuron pattern is stimulated, it arouses the other part and the name results; or, given the name, the visual image of the object results. Now the ability to make these elaborate and complicated associations, which result in such memories as we have illustrated or even more complex ones, depends upon the completeness of the development of the neuron pattern. An individual, whose neuron systems have fully developed in the brain and have been actively used in the making of thousands and millions of associations, has the mechan- 


\section{PSYCHOLOGY OF THE NORMAL AND SUBNORMAL}

ism for recalling these things that have once been in his consciousness.

On the other hand, the individual, whose brain has ceased to develop before these more elaborate neuron centres have developed, can never make these associations. It must be clearly understood that this does not mean that a person of undeveloped mind could not remember that Vladivostock is the Pacific terminal of the Trans-Siberian Railway; he can make that direct association as easily as he can make many others that sound more simple. What we are saying is, that not having that association directly available, he could never get at the goal he seeks by the roundabout associations that we illustrate.

The idiot associates the sight of food with the process of eating, he associates the sight of his attendant with certain activities and pleasures perhaps. The idiot can learn to say 7 and 6 are 13, but if he ever forgets that direct association he can in no way discover how many are 6 and 7. And of course it is perfectly clear that if he gets it, it means nothing whatever to him.

The writer has heard idiots, or at least low grade imbeciles, with a mentality of from 2 to 4, recite Psalms as long as one was willing to listen. This probably came about from the fact that under the older psychology it was thot that we trained the mind by training the memory; and we trained the memory by using it. The man in charge of this particular group of idiots, being a clergyman, naturally concluded that the best thing for the child to exercise his memory upon was the Scriptures, and consequently these children were laboriously taught to make those associations. That it had any meaning whatever for them is of course unthinkable. 


\section{MEMORY IN THE FEEBLE-MINDED}

The principle here involved also explains many of the spectacular feats of memory of young children and of the feeble-minded in the public schools. It is not uncommon to find feeble-minded children in the high schools. Now a strictly high school pupil, that is, one who can intelligently do first year high school work, is not feebleminded; but the feeble-minded child gets into the high school by virtue of his good memory. The teacher in the lower grades makes the associations for him, the child memorizes them, is able to answer the questions and pass the examinations, and so gets promoted to the high school. But he can make none of the indirect associations, he has none of the wealth of associations which underlie good judgment and reasoning processes.

There are thus all degrees of capacity for memory and recall and the person that has the highest degree, what we might for the sake of illustration call a perfect memory, will be able to reason out the most abstruse problems, provided his neuron patterns have ever been stimulated, that is to say, provided he has in mind the necessary facts. He may not be able to go directly to the solution of his problem by a simple association, but he is able to work it out by a roundabout method. To illustrate further, a certain student was asked how many cubic inches in a gallon; he did not remember, a simple association that an idiot might repeat, altho as already said, unintelligently. This student, not being able to recall the direct association, began to think if there was any way by which he could arrive at the desired number. He could not think of any association to bring this measure, but he remembered the metric system. He knew accurately the length of the meter, consequently the decimeter, consequently the liter, and therefore could find the 
number of cubic inches in the liter; he also knew that the liter was almost exactly one quart. Hence he had the answer within a fraction of a cubic inch. It may not be too much to say that no feeble-minded child of any grade could have worked out such a series of associations and have arrived at the result, even tho he had memorized the metric system and knew all the other facts that are involved in the above illustration.

Altho there is more involved and much that will have to be discussed later, it may be of value to point out here that this is one of the elements in our testing of intelligence. It is only necessary to devise a series of questions that cannot be answered by direct association but must be worked out by a process of roundabout association and then by grading these questions from the simple and direct response to the very indirect one, to determine how far along this scale the individual has gone.

For example, suppose we have this problem: "There are three men desiring to cross the river, one of them weighs 300 pounds, the other two each weigh 150 pounds. A boat is at hand but it will carry only 300 pounds safely, how will they cross the river?" Undeveloped mind at a certain stage says, "I don't know the answer," and that is all there is to it. Not knowing the answer he has no conception that it is possible, by any roundabout method of reasoning, to work out the solution. Another person may have had some experience that enables him to remember that you can take two people over and bring one person back, and he tried to apply that but, not selecting the right persons, he cannot get the solution, and so gives it up. The person with a highly developed logical memory and logical association sees at once the solution of the problem. 


\section{CREATIVE IMAGINATION}

Imagination.-In a previous chapter we discussed reproductive imagination-a reproduction of simple images. We now come to the consideration of another kind. Popularly, imagination is a symbol for the wildest vagaries of the human mind-images of things that never have been experienced, indeed never existed in the world. Furthermore it is popularly supposed that the possibilities of imagination are unlimited. This is the creative imagination of psychology, and is expressive of the possibilities of unlimited combinations of neuron patterns. It underlies invention, elaboration of thot, and all the higher mental processes. It is a very fundamental condition for intelligence and that power of adaptation to a new situation which characterizes the intelligent man.

But imagination is not unlimited in the way the popular conception holds. One can indeed create imaginary pictures to an unlimited extent, but the elements that enter into any imaginary picture are all matters of experience. In other words, one forms new associations by bringing together the neuron patterns that have already been formed in the brain, but it is impossible to put into this picture anything that has not been experienced-using experience in the broad sense to include what we have referred to as vicarious experience. I may never have seen St. Peter's Cathedral in Rome, but I have heard it described or I have seen pictures of it and thus have a kind of image with its neuron pattern. That image, such as it is, I can weave into any imaginary picture that I may wish to formulate.

The statement that one cannot imagine what he has not experienced is very easily verfied by trying to imagine a colour that one has never seen, or by attempting to draw or describe an imaginary animal. In such an experiment 
one quickly discovers that he cannot put into his imaginary picture anything, the details of which have not already been experienced. But how are these different elements brot together? How do they become associated? The answer is, either by contiguity or by similarity, more often of course the latter, since more of our adult associations are of that kind. One difference between the more intelligent and the less intelligent person is a difference in the ability to perceive the similarity, which in turn is dependent upon the elaborateness of the original neuron pattern.

Wit consists in the ability to see the similarity between things not usually thot of as similar; thus bringing together, by this discovered point of similarity, things that are incongruous and that hold the attention and interest because of their absolute newness. Punning furnishes an excellent illustration of this process. Here two incongruous things are brot together in thot by the similarity in sound of the words describing them. When the English Bible was being revised it was thot desirable to improve the passage "And they received every man a penny," because "penny" gives the wrong impression of the amount of wages paid. Some one suggested that they use the English form of the original word "Denarius," making it read, "They received every one a Denary," to which one member of the board of revision replied that he should object to having the Bible state that "they received every man a Deanery." Oliver Wendell Holmes in The Autocrat of the Breakfast Table interrupts the young man who begins to talk about capital punishment by saying, "Oh! I thot you said, 'A capital pun is.meant." " The visual pun is illustrated in all of those instances where the matter has to be seen in order to be appreciated. 
Holmes' famous pun, when he said that a man should never laugh because man's laughter is the same thing as man-slaughter, well illustrates this point.

When the association between two incongruous situations or concepts is made thru the similarty of ideas, instead of the auditory or visual words, we have wit. To quote Holmes again, he says that a pun is often called the lowest form of wit, but that is because it is the foundation of all wit. The witticism here comes about thru the appreciated similarity between the foundation and the lowest part of a structure.

All ingenuity involves constructive imagination, since it consists in perceiving the similarity between two things, one of which is a matter of experience and the other something desired. Efficiency, or the ability to solve new problems, to adapt one's self to new situations by means of past experience, consists in perceiving the similarities between two situations. In all these we have the same principle which we described under association by similarity. A new situation is presented to the senses and a neuron pattern is thereby set into activity. Some part of this pattern is the same as that which entered into a past experience. It therefore connects with the past experience and that experience comes into consciousness and becomes the solution of the present situation. The possession of the adequate neuron pattern is thus the underlying condition for all these various forms of intelligent action, and as before, the possession of this adequate neuron pattern is itself contingent upon: first, the possession of fully developed neurons; and second, the necessary experience which has combined them into a pattern.

One further question may arise in the mind of the [119] 
reader at this point, namely, how does it happen that one selects the right pattern? The new situation may bear a resemblance to a great many past experiences, most of which would be useless at the present time. The usual answer to this question is that we somehow have the power of selection, or choice. What this actually means we shall leave for a future chapter.

Let us now picture the imagnation of the defective. The reader is doubtless already able to give an adequate answer to the question, "To what extent are defectives imaginative?" since it is clear that reproductive imagination involving only the revival of images in simple neuron patterns is easily possible for all minds who have the simple patterns; and we have already pointed out that even defectives have these patterns. But since constructive or creative imagination involves elaborate association processes, with the elaborate neuron patterns which can have been developed only by extensive experience, we may expect here to find the defective decidedly lacking; and such proves to be the case.

One of the most obvious facts about defectives is their inefficiency, their inability to adapt themselves to new situations. We have given many examples and could continue them indefinitely. The feeble-minded are not inventive; they are not witty, except by accident; they never make puns except of the simplest and most obvious kinds and their jokes are of the crudest. A high grade moron was asked about his "brother." He replied, "He is my half brother, not my full brother, tho I have often seen him full." The writer was, on one occasion attempting to entertain the inmates of an institution for the feeble-minded. He happened to mention that while mountain climbing he had often eaten bean soup. These chil- 
dren had a well developed neuron pattern for bean soup, since it is an occasional article of diet in the institution. Seeing that the children appreciated this reference, the writer repeated it for their amusement a great many times in the course of the entertainment, and every time it met with increasing response. Not only that, but even to this day, after eight years, many of these defectives who heard that story still enjoy the joke; and some of them never fail to refer to it day after day whenever we meet. This fairly indicates the level of mental defectives so far as humour is concerned.

The foregoing considerations give us some very definite principles for the care and training of defectives. But this will be considered in a later chapter.

The reader has doubtless been conscious of certain difficulties in the foregoing discussion amounting almost to confusion. Not only does imagination differ very slightly from memory but both seem like association, the latter also seems like attention. It is difficult to distinguish one from the others. And indeed there is no possibility of differentiating because they are all one and the same thing. There is no better evidence that what we call memory and attention and association and imagination are only different phases of the same mental process, than just this confusion. Nevertheless it seems convenient to have the varied terminology partly because it is a matter of popular speech, and partly because it is convenient to think of mind as now memory, now imagination, now association, now attention.

There is still another mental state that we spoke of as belonging to the very nature of the nervous system; it is emotion. We have now to consider this new phase of mental life and the effect upon consciousness or mind of 


\section{PSYCHOLOGY OF THE NORMAL AND SUBNORMAL}

this extension of nerve activity into an entirely new nervous mechanism.

\section{Summary}

1. The extension of nerve action into the large association areas enormously extends memory, giving us associative memory.

2. Similarly reproductive imagination becomes supplemented by creative imagination.

3 . Wit is creative imagination-a bringing together thru association by similarity, of things not usually associated.

4. The mental defective is deficient in creative imagination as in associative memory.

5. The lack of creative imagination explains their general lack of wit and humour.

6. The recognized difficulty of clearly differentiating association, memory, attention and imagination as mental processes point strongly to the unity of mind. 


\section{CHAPTER VII}

\section{THE AFFECTIVE SIDE OF EXPERIENCE}

$W_{E}$ have so far considered only the mental processes that depend upon the activity of the cerebro-spinal nervous system. Now we turn to a wholly different phase of mind, and yet one that is not only prominent, but also very important in all mental life, since it has to do with the comfort or the discomfort, the happiness or the unhappiness of the individual.

In McDougall's (34) definition of instinct (quoted p. 38 ) he says that the inherited psycho-physical disposition determines its possessor to "pay attention to objects of a certain class, to experience an emotional excitement of a particular quality upon perceiving such an object." Our task now is to attempt to understand something of the origin and nature of that "emotional excitement."

One phase of mental life familiar to every one is the feelings, technically called the affective side, the affections. Up to the present time we have spoken of the neuron patterns as tho they were simple machines; the stimulus starts the energy which flows thru the pattern and the circuit is complete when it has led to the contraction of a muscle. We described the course of energy when something presses upon the toe and the toe is moved or the foot withdrawn. So far the process seems to be a rather complicated mechanical device for getting something done, but there is always another element. Not only is there an action in response to a stimulus, but also 


\section{PSYCHOLOGY OF THE NORMAL AND SUBNORMAL}

a certain feeling about it; there is a stronger or weaker feeling of pleasure, or of displeasure, in connection with the process. In certain cases, notably in connection with instinctive action, this feeling takes on a particular character and strength and becomes what we recognize as emotion. Every one knows better than he can describe what is meant by fear, anger, wonder and similar feelings or emotions.

Just what this feeling is or whence it arises has neven been clearly understood; but there are a few known facts which will enable us to formulate an hypothesis that will help us to clear thinking.

We would best look first at some of the stronger emotions in order to see the characteristics most fully developed. If we consider any strong emotion, such as fear or anger, we are at once struck by the fact that we have a completely new set of phenomena. It is no longer a simple case, such as we have described, of a ray of light striking upon the retina and passing to the cortex, arousing a sensation with a consequent movement, all of which we have explained by simple neuron patterns; but we have large areas of the body affected, whole systems of tissues, of muscles, of blood vessels ; and a physical effect seemingly out of all proportion to the stimulus.

Let us take fear as an example. We have the neuron pattern as already described. This may be excited directly by an external stimulus or indirectly from some other centre of the brain. But besides the simple muscular activity in response to the stimulus we may have a changed heart beat; either an acceleration or a slowing, a flushing of the face or other parts of the skin, or a paleness; perhaps a choking in the throat; the mouth becomes dry, due to a cessation of the secretion of the salivary 


\section{EMOTION}

glands. Little muscles under the skin begin to contract all over the body and we have the condition known as goose flesh. Larger muscles contract violently and we have a rigour. Respiration is affected and we breathe rapidly or else the breath comes slowly. These and other effects are among the most noticeable results. But besides these there are others. A state of fear may destroy the appetite and interfere with digestion. Sweat glands may become active and we have the cold perspiration. Organs of excretion are uncontrolled and we may have involuntary urination or defecation. The lachrymal glands may be affected and tears flow.

Not all of these effects always appear in the same individual nor are they the result of fear alone. There are great individual differences. But all the facts indicate that these emotions are somehow connected with some very fundamental functions of the human organism. In general it is agreed that unpleasant emotions interfere with normal activities of the organism, while pleasant emotions help and promote these functions. With many people digestion is markedly affected; they are thrown into a serious condition of indigestion by unpleasant emotion such as comes, for example, from the reception of bad news at meal time. Some people faint at the sight of blood; certain odours produce the same effect in others. Our language is full of expressions that indicate this, such as being paralyzed by fear, blind with rage; some cry for joy as well as for grief.

Moreover, these physical conditions aroused by the stimulus do not stop when the stimulus is removed. The indigestion produced by bad news is not at once cured when the bad news is suddenly shown to be false. The evil consequences accompanying the "seeing a ghost" 
do not always cease as soon as it is discovered that the ghost is one's friend clad in a sheet.

It is clear that we have, in the foregoing, facts that are difficult of explanation by any adaptation of the mechanism so far described. That the explanation has been difficult is attested by the fact that no subject has been so vaguely and unsatisfactorily treated by writers as the psychology of the emotions. Years ago Prof. James and about the same time Prof. Lange of Denmark gave us an hypothesis that has caused much discussion. These men made the chief element in emotion the flood of sensations coming from the great body of muscles that were, set into activity by the stimuli that would usually be considered as "causing the emotion." While having its defenders, this theory has never come into universal acceptance.

In 1899 Angelo Mosso, (41) the Italian physiologist, announced the results of some very original experiments on the effect of emotional states on the contractions of the bladder. In that connection he stated: "The seat of the emotions lies in the sympathetic nervous system."

Quite recently Cannon (8) and also Crile (11) have demonstrated physiological connections between emotion and glandular influence by way of the sympathetic system.

The extreme delicacy of the mechanism of the sympathetic system was shown by Mosso's experiments. He says: "It was sufficient to speak kindly to the animals or to caress them, to make the curve express the psychic influence upon their affective movements."

To one of his women subjects he said: "Now I am going to pinch you," but without doing so. "Immediately the bladder contracted without the slightest 

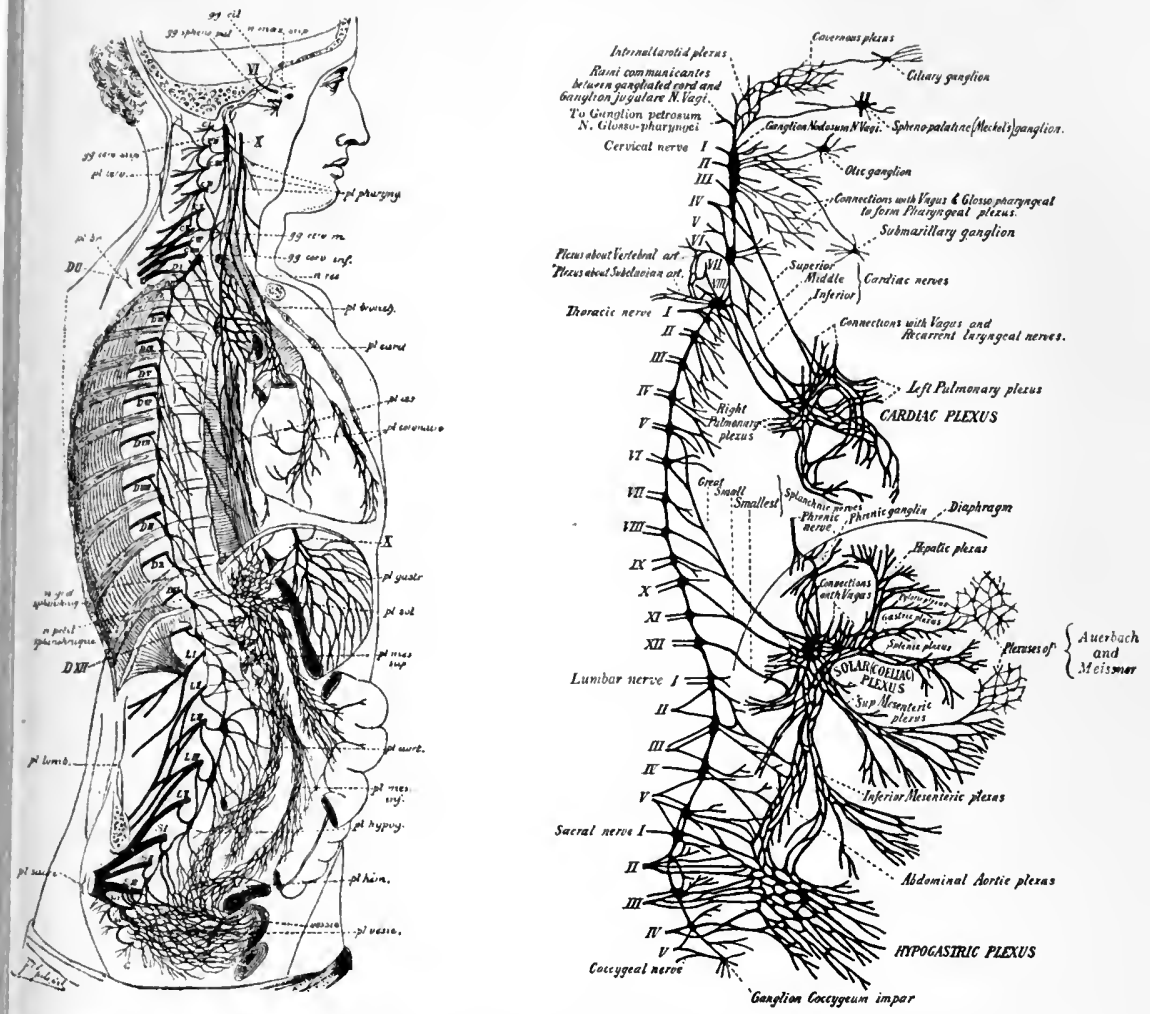

Fig. 3. A, the sympathetic system showing its location in the body.

Fig. 3. B, the same removed. The black knots in the chain are the sympathetic ganglia: the paired lines extending to the left from each ganglion are the rami communicantes or connecting branches that join the sympathetic system to the cerebro-spinal. This clain is just outside the spinal column.

From Van Gehuchten'after Schwabbe 



\section{EMOTION AND THE SYMPATHETIC SYSTEM}

change in the thoracic and abdominal respiration." This simple arithmetical problem: "How many eggs are 7 dozen?" produced the same result. He sums it up by saying: "We understand now that the constant and fundamental movements taking place in emotions are the movements of the internal organ of vegetative life. This end could be obtained only thru the sympathetic system."

We must therefore extend our view of neuron action beyond the cerebro-spinal system, to which we have so far confined ourselves, into the great and intricate sympathetic system.

Science has not yet explained many of the details of the way the sympathetic system functions, but we may assume certain hypotheses which will give us a working plan.

We have shown, Fig. 3, that the sympathetic is connected with the cerebro-spinal system at every vertebra along the spinal stem by connecting branches, the rami communicantes. The white ramus conveys outgoing stimuli, the grey ramus, the incoming. The neurons from each of the two systems pass thru these rami into the other system, thus making the connection complete. It was pointed out that the neurons of the spinal cord, thru their branches, were in connection with a great many different neurons. Among these different neurons are those of the sympathetic system; so that it is easy to uderstand that a stimulus coming in over a sensory neuron, passing to various parts of the cord or up to the cortex, also sends some of the energy by one of its branches into the sympathetic system.

Moreover, comparative anatomy of the nervous system shows that the sympathetic is the oldest of all nervous structures and therefore it is safe to assume that the 


\section{PSYCHOLOGY OF THE NORMAL AND SUBNORMAL}

neuron patterns here are practically all inherited. Therefore the neurokyme flows naturally and easily into the sympathetic system and in accordance with the theory of development we may conclude that it produces such action as is beneficial for the individual or the race. Fig. 3 gives some idea of the elaborateness of the sympathetic system, the enormous extent to which it ramifies to the internal organs and glands.

We must now go a little more into detail. The following is condensed from Cannon (8):

"The skeletal muscles receive their nerve supply directly from the central nervous system. The neurons distributed to these muscles are parts of neurons whose cell bodies lie within the brain or spinal cord. The glands and smooth muscles of the viscera, on the contrary, are, so far as is now known, never innervated directly irom the central nervous system. The neurons reaching out from the brain or spinal cord never come into immediate relation with the gland or smooth-muscle cells; there are always interposed between the cerebrospinal neurons and the viscera extra neurons whose bodies and processes lie wholly outside the central nervous system. They are represented in dotted lines in Fig. 41. I have suggested that possibly these outlying neurons acted as 'transformers,' modifying the impulses received from the central source (impulses suited to call forth the quick responses of skeletal muscle), and adapting these impulses to the peculiar, more slowly-acting tissues, the secreting cells and visceral muscle, to which they are distributed.

"The outlying neurons typically have their cell bodies [128] 


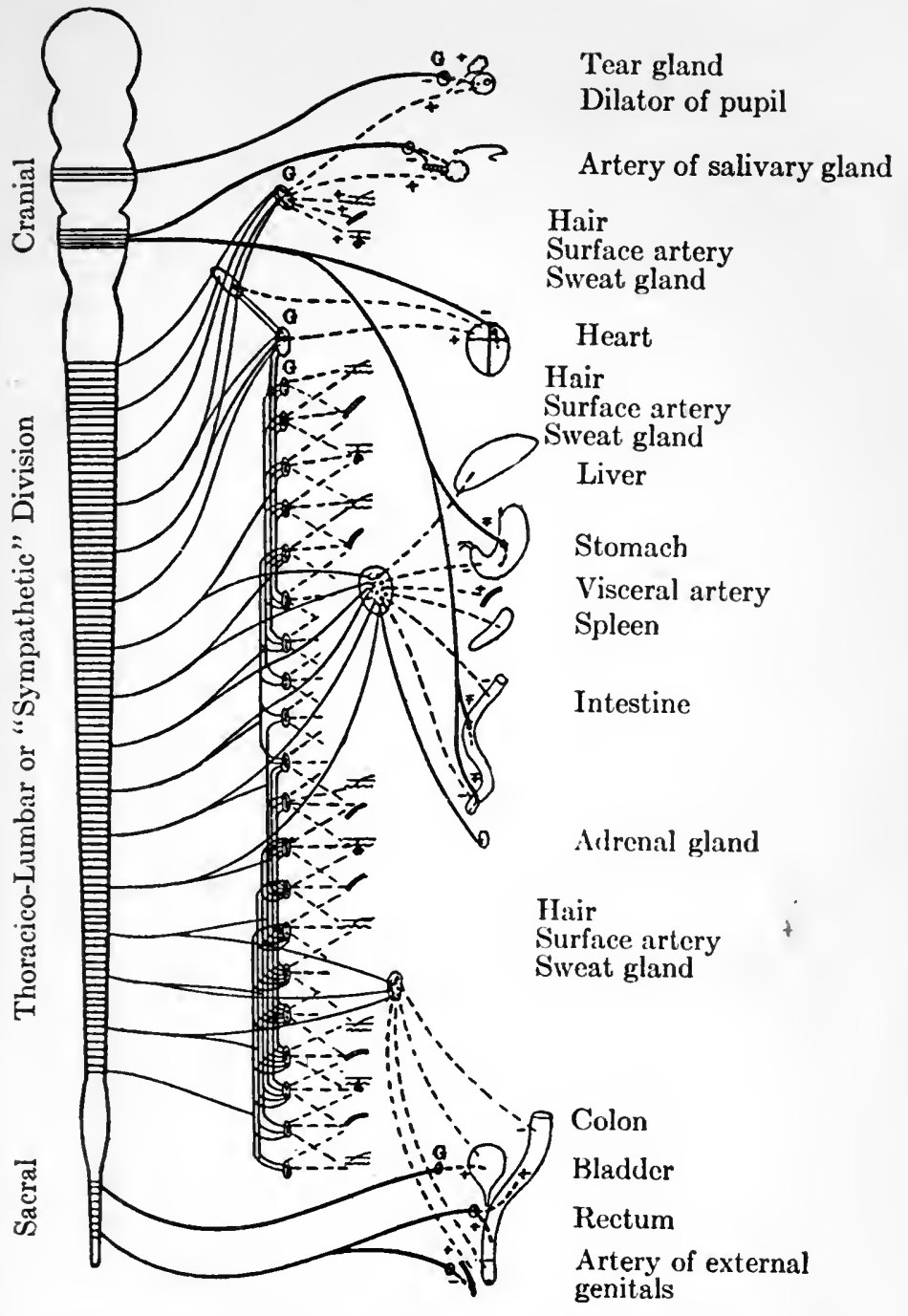

Fig. 41. Diagram of the more important distributions of the autonomie nervous system The brain and spinal cord are represented at the left. Note the little plus and minus signs, + indicates stimulating effect; - indicates inlibitory effect. 



\section{CANNON QUOTED}

grouped in ganglia (G's, Fig. 41) which, in the trunk region, lie along either side of the spinal cord and in the head region and in the pelvic part of the abdominal cavity are disposed near the organs which the neurons supply. In some instances these neurons lie wholly within the structure which they innervate (see e.g., the heart and the stomach, Fig. 41). In other instances the fibres passing out from the ganglia-the so-called 'postganglionic fibres'-may traverse long distances before reaching their destination. The innervation of blood vessels in the foot by neurons whose cell bodies are in the lower trunk region is an example of this extensive distribution of the fibres.

"As suggested above, the outlying neurons are connected with the brain and spinal cord by neurons whose cell bodies lie within the central nervous organs. These connecting neurons, represented in continuous lines in Fig. 41, do not pass out in a continuous series all along the cerebrospinal axis. Where the nerves pass out from the spinal cord to the fore and hind limbs, fibres are not given off to the ganglia. Thus these connecting or 'preganglionic' fibres are separated into three divisions. In front of the nerve roots for the fore limbs is the head or cranial division, between the nerve roots for the fore limbs and those for the hind limbs is the trunk division (or thoradico-lumbar division, or, in the older terminology, the 'sympathetic system') ; and after the nerve roots for the hind limbs the sacral division.

"This system of outlying neurons, with postganglionic fibres innervating the viscera, and with preganglionic fibres reaching out to them from the cerebrospinal system, has been called by Langley, to whom we are in- 


\section{PSYCHOLOGY OF THE NORMAL AND SUBNORMAL}

debted for most of our knowledge of its organization, the autonomic nervous system. ${ }^{1}$ This term indicates that the structures which the system supplies are not subject to voluntary control, but operate to a large degree independently. As we have seen, a highly potent mode of influencing these structures is thru conditions of pain and emotional excitement. The parts of the autonomic system-the cranial, the sympathetic, and the sacral-have a number of peculiarities which are of prime importance in accounting for the bodily manifestations of such affective states.

"The fibres of the sympathetic division differ from those of the other two divisions in being distributed thru the body very widely. They go to the eyes, causing dilation of the pupils. They go to the heart and, when stimulated, they cause it to beat rapidly. They carry impulses to arteries and arterioles of the skin, the abdominal viscera, and other parts, keeping the smooth muscles of the vessel walls in a state of slight contraction or tone, and thus serving to maintain an arterial pressure sufficiently high to meet sudden demands in any special region; or, in times of special discharge of impulses, to increase the tone and thus also the arterial pressure. They are distributed extensively to the smooth muscle attached to the hairs; and when they cause this muscle to contract, the hairs are erected. They go to sweat glands, causing the outpouring of sweat. These fibres pass also to the entire length of the gastro-intestinal canal. And the inhibiton of digestive activity which, as we have learned, occurs in pain and emotional states, is due to impulses

1 What we, following the popular terminology, have called the sympathetic system, Cannon here calls the autonomic nervous system; he moreover divides it into three parts, the cervical, the sympathetic and the sacral autonomic nervous systems. 


\section{CANNON QUOTED}

which are conducted outward by the splanchnic nervesthe preganglionic fibres that reach to the great ganglia in the upper abdomen (see Fig. 41) -and thence are spread by postganglionic fibres all along the gut. They innervate likewise the genito-urinary tracts, causing a contraction of the smooth muscle of the internal genital organs, and usually relaxation of the bladder. Finally they affect the liver, releasing the storage of material there in a manner which may be of great service to the body in time of need. The extensiveness of the distribution of the fibres of the sympathetic division is one of its most prominent characteristics.

"The cranial and sacral autonomic divisions differ from the sympathetic in having only restricted distribution (see Fig. 41). The third cranial nerves deliver impulses from the brain to ganglia in which lie the cell bodies of neurons innervating smooth muscle only in the front of the eyes. The vagus nerves are distributed to the lungs, heart, stomach, and small intestine. As shown diagrammatically in Fig. 41, the outlying neurons in the last three of these organs lie within the organs themselves.

"In this sacral division the preganglionic fibres pass out from the spinal cord to ganglia lying in close proximity to the distal colon, the bladder, and the external genitals. And the postganglionic fibres deliver the nerve impulses only to the near-by organs. Besides these innervations the cranial and sacral divisions supply individual arteries with 'dilator nerves'-nerves causing relaxation of the particular vessels.

"The cranial autonomic, represented by the vagus nerves, is the part of the visceral nervous system concerned in the psychic secretion of the gastric juice. Pav- 


\section{PSYCHOLOGY OF THE NORMAL AND SUBNORMAL}

lov showed that when these nerves are severed psychic secretion is abolished. The cranial nerves to the salivary glands are similarly the agents for psychic secretion in these organs, and are known to cause also dilation of the arteries supplying the glands, so that during activity the glands receive a more abundant flow of blood.

"Great emotion, such as is accompanied by nervous discharges via the sympathetic division, may also be accompanied by discharges via the sacral fibres. The involuntary voiding of the bladder and lower gut at times of violent mental stress is well-known. Veterans of wars testify that just before the beginning of a battle many of the men have had to retire temporarily from the firing line. And the power of sights and smells and libidinous thoughts to disturb the regions controlled by the nervi erigentes proves that this part of the autonomic system also has its peculiar affective states.

"Lying anterior to each kidney is a small body-the adrenal gland. It is composed of an external portion or cortex, and a central portion or medulla. From the medulla can be extracted a substance, called variously suprarenin, adrenin, epinephrin or "adrenalin," which, in extraordinarily minute amounts, affects the structures innervated by the sympathetic division of the autonomic system precisely as if they were receiving nervous impulses. For example, when adrenin is injected into the blood it will cause pupils to dilate, hairs to stand erect, blood vessels to be constricted, the activities of the alimentary canal to be inhibited, and sugar to be liberated from the liver. These effects are not produced by action of the substance on the central nervous system, but by direct action on the organ itself. And the effects occur 


\section{CANNON QUOTED}

even after the structures have been removed from the body and kept alive artificially.

"The adrenals are glands of internal secretion, i.e., like the thyroid, parathyroid, and pituitary glands, for example; they have no connection with the surface of the body, and they give out into the blood the material which they elaborate.

"The foregoing brief sketch of the organization of the autonomic system brings out a number of points that should be of importance as bearing on the nature of the emotions which manifest themselves in the operations of this system. Thus it is highly probable that the sympathetic division, because arranged for diffuse discharge, is likely to be brought into activity as a whole, whereas the sacral and cranial divisions, arranged for particular action on separate organs, may operate in parts. Also, because antagonisms exist between the middle and either end division of the autonomic, effective states may be classified according to their expression in the middle or an end division and these states would be, like the nerves, antagonistic in character. And finally, since the adrenal glands are innervated by autonomic fibres of the mid-division, and since adrenal secretion stimulates the same activities that are stimulated nervously by this division, it is possible that disturbances in the realm of the sympathetic, although initiated by nervous discharge, are automatically augmented and prolonged thru chemical effects of the adrenal secretion.

"That the adrenal glands are subject to splanchnic influence has been demonstrated anatomically and by the physiological effects of their secretion after artificial stimulation of the splanchnic nerves. Impulses are nor- 


\section{PSYCHOLOGY OF THE NORMAL AND SUBNORMAL}

mally sent along these nerves, in the natural conditions of life, when animals become greatly excited, as in fear and rage and pain. There is every probability, therefore, that these glands are stimulated to extra secretion at such times. Both by an exceedingly delicate biological test (intestinal muscle) and by an examination of the glands themselves, clear evidence has been secured that in pain and deep emotion the glands do, in fact, pour out an excess of adrenin into the circulating blood.

"Here, then, is a remarkable group of phenomena-a pair of glands stimulated to activity in times of strong excitement and by such nerve impulses as themselves produce, produce at such times profound changes in the viscera; and a secretion given forth into the blood stream by these glands, which is capable of inducing by itself, or of augmenting, the nervous influences which induce the very changes in the viscera which accompany suffering and the major emotions."

Cannon has shown that a stimulation of the adrenal gland produces all the phenomena of anger and rage, and conversely that production of anger or rage by any other method effects the secretions of the adrenal glands; and we know that the phenomena above enumerated are controlled by the sympathetic system.

Cannon worked with adrenin, the secretion of the adrenal gland. This is one of the glands of internal secretion. There are a number of other such glandsthe thyroid, thymus, pineal, pituitary, parathyroid, carotid and coccygeal, areas of Langerhaus of the pancreas, interstitial cells of the testicle and corpora lutea of the ovary.

There is no reason to doubt that some, if not all, of [134] 


\section{THE FEELINGS}

these other glands may have similar relations to emotion.

It is clear from the foregoing quotation that we have in the sympathetic system a nervous mechanism little if any inferior to the cerebrospinal, and fully capable of producing all the phenomena of the affective life.

We are now ready to complete our working plan of emotional activity. Every stimulus to a sensory neuron arouses nerve energy which runs into the nerve pattern as already described. But a part of this energy passes over the rami communicantes into the sympathetic system. The sympathetic system, thus set into activity, influences more or less strongly the glands of internal secretion and other organs. The activities thus aroused result in various modifications of function which are for the benefit of the individual. If the original stimulus is of a character to be injurious, one type of activity (special set of glands e.g.) results; if beneficial, another type of response occurs; but always with a tendency toward the final welfare of the organism. These activities are reported back to the central nervous system accompanied by more or less of consciousness.

We may readily assume that there is always a minimum of consciousness which ordinarily does not come strongly to the surface, that is, arouse attention, because it is usually followed by some more direct effect on the cortical neuron pattern aroused by the original stimulus. A mild feeling of satisfaction or comfort is the common result of ordinary activity; nevertheless, it may at any time come into the focus of consciousness, in which case we speak of the feeling accompanying the situation. For example, the sight of food to a hungry man may, so far as consciousness is concerned, lead only to his active move- 
ment to secure such food; but it may also give him a very distinct feeling of pleasure. The stimulation of the auditory nerve by an unusual sound may lead exclusively, so far as consciousness is concerned, to movements calculated to discover the origin of the sound. But it may also lead to a very distinct feeling of fear or, if it is recognized as a sound associated with some pleasing object, the feeling may be one of pleasure.

All such experiences of satisfaction, mild pleasure, and on the other hand moderate anxiety, worry and fear, constitute what are usually called the feelings and must be thought of, as already stated, as resulting from a moderate inflow of energy from the sympathetic system which in turn resulted from a moderate overflow into that system from the neuron pattern aroused by the original stimulus. One of the feelings that has received a definite name, and consequently will serve us as a further illustration of this mild degree of the affective consciousness, is interest.

What we call interest is only the feeling accompanying an ordinary state of attention. It is a contribution of the sympathetic system to the work of the cerebrospinal system. As we have said, every state of consciousness has this feeling side. The old question formerly so learnedly discussed at Teachers' Institutes as to which is first, attention or interest; whether the teacher should strive to interest her pupils and thereby make them attentive or whether she should vigorously require attention, and they would thus become interested, has long since been shown to be one of those fanciful problems born of ignorance.

Attention and interest are but two phases of the same mental experience. One does not exist without the 


\section{INTEREST AND ATTENTION}

other. Sometimes one is a little more in evidence than the other and then we are apt to describe the state of consciousness in terms of that phase which is more prominent. If the feeling side is predominant we describe a particular experience as interesting, if on the other hand the attention is the stronger phase, as evidenced by the bodily expressions of attention, the feeling of effort as we try to keep the matter in consciousness, then we describe the experience as one to which we give. our best attention.

We also apply these terms when we observe the conduct of another. Interest is a pleasant feeling and is shown in certain facial expressions which always accompany pleasant emotions. Therefore, if we see these expressions of countenance, we are apt to say the person is interested. On the other hand the attentive state of consciousness manifests itself in certain muscular strains and bodily attitudes, which, being just as easily recognized as the other, enable us to say that the person paid strict attention. From a practical standpoint it makes little difference which phase we observe since we know whichever one is observed the other is present.

When the original stimulus is a very violent one, or, because of inherited tendencies or acquired experiences, arouses a very elaborate neuron pattern in the central system, a vastly greater flow of energy into the sympathetic takes place, resulting in rather serious disturbance of the organs and glands controlled by that system. For instance, when the adrenal gland is affected and its secretion is poured into the blood, we get decided and unusual activities of the heart, the liver and other organs, which violent activity is again transmitted over the other ramus into the central system with an accompanying in- 


\section{PSYCHOLOGY OF THE NORMAL AND SUBNORMAL}

tense consciousness which we know as the emotion, either of fear which may amount to terror, or of anger which may rise to the height of rage. It can be shown, as Cannon has done, that all this is for the benefit of the individual. The fear leads him to run away, the rage impels him to fight; either movement calls for an unusual amount of energy on his part. The effect of the adrenal secretion has been to quickly deposit energy, in the form of sugar, in the blood for the use of the various muscles and organs involved; for the control of the products of fatigue; and for the rapid coagulation of the blood which may easily be caused to flow as the result of the combat that the anger may lead to, especially in primitive man and animals.

If this hypothetical picture is even roughly correct it at once becomes clear that the James-Lange theory of emotions is true so far as it goes, but that it is only part of the story because it confines itself to the central nervous system and does not take into account the enormous part played by the sympathetic. It required a Mosso to show us that such organs as the bladder do actually respond to situations that give rise to emotions; and then a Cannon to complete the picture by demonstrating to us that the secretions of the glands, which are innervated by the sympathetic, react quickly upon the various organs, thus producing the incoming sensation which James and Lange were the first to recognize.

It is convenient to consider the affective side of consciousness in two parts as we did the action side. First, that which goes with the activity of inierited neurons (including such acquired patterns as are formed by simple association neurons within the localized centre, or between the cortical centres); and, second, those activi-

[138] 


\section{SUMMARY}

ties resulting from the participation of the great association areas. Just as this participation gave us more extended attention, memory and imagination, so it gives us more elaborate emotional states. These will be considered in the next chapter.

\section{Summary.}

1. The mental processes discussed in the previous chapters belong to the sensation side of consciousness and are conditioned by the structure and functioning of the cerebrospinal nervous system.

2. There is another side of consciousness known as the affective side-the feelings.

3. Strong feelings are called emotions and every primary instinct is accompanied by its characteristic emotion.

4. "The seat of the emotions lies in the sympathetic nervous system."

5. The glands of internal secretion are apparently important factors in the production of emotion.

6 . When the affective phase of consciousness is pleasant, but falls short of an emotion in intensity, it is called interest. Since we speak of the consciousness aroused by any particular stimulus, or group of associated stimuli, as a state of attention, it is frequently said that interest and attention are two phases of the same consciousness.

7. The extension of nerve action into the great association areas of the brain results in "higher emotions" just as we had second or higher types of memory, association, imagination, etc. 


\section{CHAPTER VIII \\ COMPLEX EMOTIONS}

IT is a necessary consequence of the extension of the association process, by the involvement of the large association areas of the brain, that experience should bring about extensive association between the instincts. Whereas each instinct taken by itself is accompanied by its specific affective quality, that is its own emotion, when two or more instincts are aroused at the same time there will naturally result a much more complicated emotional state.

Since Des Cartes, emotions have been divided into primary emotions, those accompanying the primary instincts, and complex emotions. The accompanying diagram, Fig. 42, prepared by the writer from McDougall's (34) description of instinct and emotion, shows in graphic form his scheme. Within the rectangles we have in the opper line the primary instinct and in the lower line the corresponding emotion. Below this, at the point of convergence of the various oblique lines we have the complex emotions. For example, when the instinct of flight is associated with the instinct of repulsion we have the emotion of fear combined with that of disgust, which results in the emotion of loathing. When to this is added the instinct of curiosity with its feeling of wonder we get fascination. Repulsion with pugnacity gives scorn. Curiosity with self-abasement gives admiration. Add to this the instinct of flight and we get awe. The parental instinct combined with self-abase- 


\section{COMPLEX EMOTIONS}

ment gives gratitude and when this is added to that which gives awe we have reverence; similarly with the other combinations indicated.

It is interesting to note that it seems possible to analyse these higher emotions into their elements by process of introspection. For instance, it seems that one can perceive that reverence is a combination of awe and gratitude, and gratitude a combination of the feeling of subjection and the tender emotion, and so on. It would, however, seem very doubtful whether psychologically we ought to say that reverence is a combination of awe and gratitude, and not rather that it is a complex emotion resulting from an intricate association between the parental instinct, self-abasement, flight and curiosity.

There is little agreement among psychologists as to what should be considered the fundamental instincts or what should be included in the list of emotions. But we may accept provisionally this scheme of McDougall's and leave it for future investigation to revise by extension or reduction.

That these complex emotions are the result of extensive associations between the primary centres is suggested if not proved by certain facts; such as the noticeable decrease of these emotions as we get into lower forms where the association centres are developed little or not at all. Those primary instincts which are most naturally brot together by the environment have developed complex emotions farther down the series than have those instincts less often brot together. For example, flight and curiosity are very often aroused at the same time even in animals, with the result that fascination is quite common among them and in children. From this it may be concluded that there is an inherited 


\section{PSYCHOLOGY OF THE NORMAL AND SUBNORMAL}

neuron pattern bringing together the neurons involved in these instincts.

On the other hand, no one would ascribe reverence to any animal or even to young children. The reason is clear. The four instincts involved do not often come together in animal or child experience. It may be a question whether loathing, scorn or admiration can be attributed to animals. Of the three it may be that admiration develops earlier in the child than the other two. Contempt would seem to have a very late development in childhood and is probably absent from animals. Likewise reproach, altho certain animals, for example, the dog, do have attitudes and expressions of eyes that we are inclined to say indicate reproach.

Speaking in the same broad and general way that we have done before, we may say that the simple primary emotions are characteristic of animals and undeveloped humans, while the complex emotions are characteristic of mature or fully developed human beings. From our neurological point of view this would mean that beings without well developed cortical association areas cannot have the complex emotions.

We have still to consider a plausible tho hypothetical picture of the nerve action underlying the complex emotions. If the situation which arouses the instinct of flight starts a neuron pattern from which a certain amount of energy overflows into the sympathetic system and there liberates more nervous energy or causes certain glands to secrete, and these secretions further stimulate other glands and smooth muscle, and this augmented energy then flows back into the muscles and shows itself in consciousness as the emotion of fear or terror; and if at the same time a situation arouses the instinct 


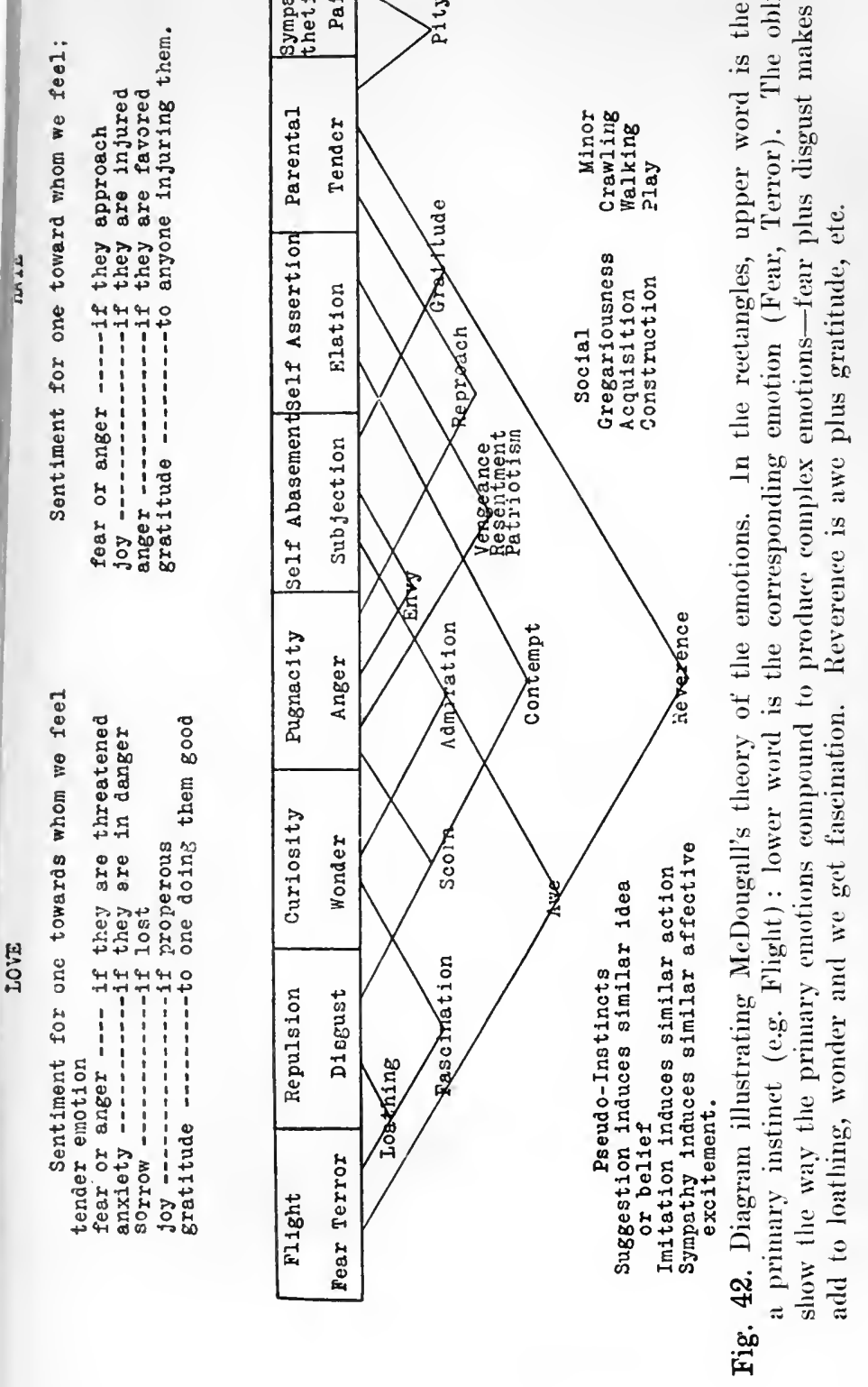





\section{NEUROLOGY OF EMOTIONS}

of repulsion which in a similar manner causes an overflow to and from the sympathetic system, resulting in the emotion of disgust, it is not difficult to imagine that when both of these instincts are aroused at once, the sympathetic system would be either more strongly aroused or at least very differently aroused; and as a consequence the muscular system would be differently affected and also there would be a different quality of consciousness, a new emotion.

It is easy to see that the presence at the same time of the specific stimuli for the two instincts may lead to an interference, a blocking, which may result in an extension of the neuron patterns into the large association areas. This of itself would lead to an increase of nerve energy which we might expect would overflow into the sympathetic system with results already described.

At first sight it seems a little difficult to explain why the complex emotion would not always be much more violent than the simple emotion. For, given the extensive association between two fundamental instincts resulting in a very much enlarged and complicated neuron pattern in the brain, we might expect a greater overflow into the sympathetic system with consequent greater disturbance there and greater returns and greater muscular activity and consciousness. For all we know this may be literally true and while we cannot say that loathing, for example, is always a stronger emotion than disgust or fear, it may be true that a situation that would arouse a certain degree of fear, combined with a situation that would arouse a certain degree of disgust, would when combined arouse a degree of loathing that would be vastly greater than either the fear or the disgust.

In other words, our feeling that the emotion of loath[143] 
ing is not necessarily more intense than the emotion of disgust may be the result of comparing two very different situations. The case where the disgust is stronger than the loathing may be the result of a situation where the stimulus is enormous, while the loathing may result from a situation where the stimuli to the instincts of repulsion and flight are very mild; similarly, of course, for the other emotions.

But this is not all; we may properly assume that there are certain compensations or neutralizing effects. To take a gross illustration, one instinct or its emotion may cause a certain gland to secrete, the secretion of which gland would result in a tremendous activity of the sympathetic system; but the other emotion acting at the same time may cause the secretion from some other gland to flow and the effect might be to actually neutralize the first, or perhaps to form in the blood a new compound whose action is quite different from that of either of the originals. That there is antagonism between different parts of the sympathetic (autonomic) system has been shown by Cannon. It will thus be seen that there are almost infinite possibilities.

In brief, then, we see that the higher or complex emotions are dependent upon the development of the great association areas for the mechanism necessary to bring together the separate experiences, which but for such connection would produce only a series of simple emotions. Moreover, the entire affective phase of mind is dependent upon the sympathetic nervous system for that particular quality which characterizes feeling. It is of interest to note that the relative independence of the sympathetic nervous system from the cerebrospinal nervous system parallels the common result of introspec- 
tion that feelings and emotions are somehow distinct from the other phases of mind.

Again it is the feeble-minded who have given us this concept and whose condition is most clearly explained by this view. All the primary instincts with their accompanying emotions according to McDougall's list, are present in the feeble-minded. Many of these at first sight seem to be over-developed, but that is probably in appearance only, the appearance being due to the lack of control.

Flight with its emotion of fear is found well down the scale, possibly even to the lowest.

Repulsion with its emotion of disgust is clearly present in all but the lowest grade. Here it would seem to be absent, especially in that function from which the emotion takes its name, namely the gustatory sense, since idiots are known to eat most disgusting things.

Curiosity (wonder) is also noticeably less as we go down the scale. Indeed, it would seem that even the morons are possessed of less of this instinct than the normal child.

Pugnacity and anger are in evidence in all grades.

Self-abasement tho present is not so manifest as some of the others.

Self-assertion on the other hand is prominent, indeed it is so well developed in the defectives that it is one of the most useful instincts in connection with their training. They can be induced to do a great many things that they would not otherwise undertake by the knowledge that they will have an opportunity to "show off," to assert themselves.

The parental instinct with its tender emotion is clearly developed in the chronologically older cases, and extends 


\section{PSYCHOLOGY OF THE NORMAL AND SUBNORMAL}

fairly well down the mental scale. Morons, both boys and girls, delight to take care of the younger children and then manifest toward them what seems to be real parental feeling. It is evident that this instinct, like some of the others, cannot be manifested by the lowest grade, even tho it may be present in rudimentary form.

Sympathy, or what McDougall calls sympathetic pain is also manifest in the higher grades.

A question arises as to why these different instincts, if they are primary, which, according to McDougall, means that they are present in animals as well as in humans, should not be all equally manifest in even the low grade feeble-minded. It is quite possible that they are present, indeed if one reasons a priori one must conclude that they are present just as in normal children. But they are not so much in evidence, because in the first place the specific stimulus for each emotion is not so often applied to defectives; and our understanding of these instincts in normal people is largely influenced by our observation of their instinctive reactions, not to the original, the specific stimulus, but to associated stimuli.

McDougall shows that in normal people these instincts are modified in various ways. The instincts, according to him, are normally modified on the afferent or receptive side, that is to say, in the kind of stimulus that can arouse the instinct. One method is by "specialization." For instance, primitively any loud noise will excite the emotion of fear, but with experience one learns that some loud noises are not causes for fear. There is also the opposite process of an extension of stimuli by association. If the specific stimulus for the instinct is constantly associated with some other stimulus, it will not be long until the 


\section{MODIFICATION OF INSTINCTS}

second stimulus starts the instinct just as the original one did. This is the so called conditioned reflex and Mateer (37) has shown that it can be developed even in the lowest idiots.

Moreover, this association between the specific stimulus and some associated stimulus may be by contiguity and also by similarity with the same differences that we have noted in other connections; contiguity being much more common and direct, but similarity having a wider range.

Now it is evident in the first place that the feebleminded will not modify their instincts as will normal people; and secondly, and what is more important, there will be a great difference in the extent to which the different instincts are modified. The instinct for flight, the emotion of fear, will be slightly modified, because in the very nature of the case the yielding to the impulse to run away from the stimulus takes away the opportunity of discovering that the stimulus is not properly fearful. The defective will not use association by similarity here any more than elsewhere, and association by contiguity in the way of substituting new stimuli as arousers of the instinct would be practically the only way in which it would be modified and we may imagine that the effect of this would be slight.

As a matter of fact we find that the feebleminded show about a normal amount of fear until we get up to those things that depend upon intelligence. The feeble-minded do not fear microbes and germs because they have not enough intelligence to understand them. They do fear loud noises and bright lights and grotesque appearances and strangers. Idiots and imbeciles do not fear the lower forms of animal life, except when they are in active 


\section{PSYCHOLOGY OF THE NORMAL AND SUBNORMAL}

motion. The morons have evidently learned these fears from more intelligent people. For example, low grade imbeciles and idiots have no fear of snakes. From this it is evident that the so-called instinctive fear of snakes is not instinctive but acquired.

Disgust is primitively the emotion that accompanies the repulsion of something that does not taste right, but the term has come to have a figurative use. We speak of certain kinds of conduct as being disgusting or even of disgusting ideas. Expression of disgust in this sense is of course not to be thot of with idiots or imbeciles and only slightly with morons. Low grade defectives, being somewhat deficient in the sense of taste, do not display the repulsion even for those things that the adult finds disgusting in the literal sense of the term. Moreover, this instinct is enormously extended in normal people thru association by similarity.

Still confining ourselves to the original meaning, relating to food, the extent to which a thing may become disgusting because it looks similar to something that is known to be distasteful is well recognized. Contiguity also plays a part with normal people, which it undoubtedly does not do with defectives. For instance, if compelled to eat in a filthy place, or if while eating in a comfortable place, some disagreeable scene has transpired, it is quite likely that one will not be able to eat the same things even under favourable conditions. The emotion of disgust is aroused by associations with the previous experience.

Curiosity is another instinct that is greatly elaborated in normal people thru association by similarity. Consequently we would expect it to be, as we find it, much less manifest in defectives. 
Pugnacity on the other hand, or the emotion of anger, is one of the few emotions that remains fairly constant. It is mainly aroused by its specific stimulus. As a result this instinct appears normally developed in defectives.

In self-abasement we have another instinct that is largely modified and in a normal person many stimuli not naturally specific for self-abasement come to produce the instinct. With the feeble-minded this is not the case, consequently self-abasement is less often in evidence. On the other hand, the instinct for self-assertion is in the normal person modified in the direction of suppression. The child is early taught modesty, and the stimuli that would naturally arouse this instinct are turned into other channels so that these self-assertions are not so prominent. With the feeble-minded, on the other hand, as already said, this is used as an exceedingly useful instinct in their training, development and discipline.

McDougall (34) also points out that instincts are normally modified on the conative side, that is to say, in the form of the action as the expression of the instinct. Here again there is a marked difference between the normal and the defective. The normal person early learns to control some instincts and to extend others. He acts upon the one, but fails to act upon the other because the consequences are unpleasant. The feebleminded because of their weak association processes are much less liable to learn these methods of control.

Another influence to be considered is the conflict of emotion. A casual glance shows that some of these emotions are contradictory to others and if the stimuli for two opposite emotions should come at the same time, there would at once be a conflict. But the possibility for this conflict depends upon there being a reasonably 
strong association between the two. If there is no such association, the one instinct is acted upon and the other dies out. We can see therefore why it is that these conflicts occur more in normal people than in defectives, since the latter do not have the necessary association neurons. For instance, a normal child quickly learns to control his fear because of his pride. Fear and curiosity. or wonder are also somewhat antagonistic. In the case of the normal child his whole effort is to control fear and to extend curiosity.

We said in the previous chapter that a strong stimulus, or one that aroused an inherent pattern or an elaborate. acquired pattern, would cause a heavy overflow into the sympathetic system with a resulting violent emotion. It is a familiar fact that violent emotion is often aroused by an extremely insignificant stimulus. A single word innocently uttered may call forth intense rage or profound fear. A sound that would pass unnoticed by most persons may in one with the right experience produce all the outward manifestations of terror; likewise a visual stimulus or an odour, or in fact, almost any stimulus whatever. The western cowboy jumped to his saddle and rode for his life upon the perception of a certain sound which was utterly ignored by the inexperienced. He had heard the ominous sound which to him meant a prairie fire. On one occasion the writer was sitting comfortably before an open fire in a hotel in the Canadian Rockies, chatting pleasantly with his Swiss guide. He mentioned the fact that he had climbed the Jungfrau just ten days after a notorious accident in which several tourists and guides had been killed. Instantly the guide turned pale and showed all the outward signs of intense pain and fear. As soon as he could get his voice he told 
us that he was one of the two guides who had been in that catastrophe and had escaped.

In both these instances elaborate neuron patterns had been built up by experience. The sound or the word was enough to start into activity this pattern with all its multitude of associatons. From numerous neurons, energy passed over into the sympathetic system arousing that to strong activity, causing glandular secretions, the effect of which was to produce the recognized physical effect of the emotion.

How this is brot about in the neuron system may be better understood by reference to what Cajal (23, p. 101) has called "Avalanche Conduction." The accompanying diagram, Fig. 43, will make this clear. The neuron patterns involved in these cases have been built up by experience. Consequently a person without such an experience cannot have this consciousness or the emotion.

The well known apathy of undeveloped or diseased

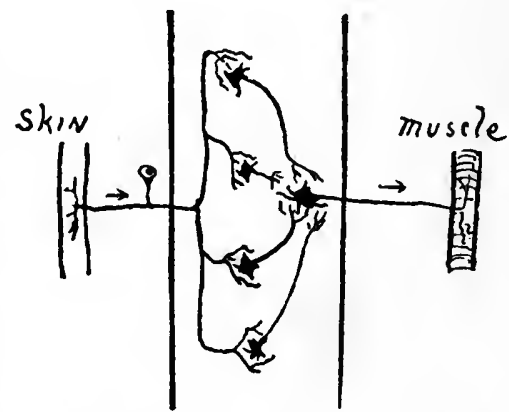

Fig. 43. Diagram of the mechanism of reinforcement whereby a single weak afferent nervous impulse may be received by several neurons of the second order which discharge their greatly strengthened nervous impulses into a single final path.

The final path might be a ramus communicans conveying the neurokyme to the sympathetic system.

From Herrick. mind is probably to be explained by the foregoing principle. A situation, a conversation or a word that regularly arouses strong emotion in an experienced adult, arouses none in the defective because he has no elaborate neuron pattern to be aroused, and consequently no great overflow into the sympathetic system. One of the children of a certain institution, who is probably more 
a case of mild insanity than of arrested development, recently said to the writer, "I am very unhappy, I am homesick, I am very miserable all the time." Evidently from his words he was very sincere, but thruout the whole expression he had a pleasant smile on his countenance and a quiet natural tone of voice. He was only using language that he had heard. There were no elaborate neuron patterns underlying it and consequently no emotions.

Juries and judges are often nonplussed by the lack of emotion in certain criminals brot before them who have confessed their guilt but who can talk about the circumstances as nonchalantly as one speaks about the ordinary affairs of the day. Two murderers, studied by the writer (20a) each displayed this condition to a remarkable degree. Even the photographs of these criminals taken in prison show a smile on each of their faces. Such a state of mind amazes the inexperienced observer and he says, "What a hardened criminal. How can he be so heartless, and have so little feeling?" The explanation is entirely simple. These murderers are both feebleminded and as such have very incomplete neuron patterns. The circumstances as they are narrated do not arouse any great activity; there is therefore none of that disturbance of the sympathetic system which results in strong emotion. They can therefore contemplate any phase of the subject without any feeling that expresses itself in the bodily symptoms of emotion or even in their own consciousness. While the second murderer referred to above was in prison and after he had confessed his crime he was, as the jailer remarked, more concerned as to how he should collect sixty cents that somebody owed him than he was about his own punishment or [152] 


\section{THE EMOTIONS OF DEFECTIVES}

anything connected with the crime. Similarly of the other boy we have recorded $(20 \mathrm{a}$, p. 31$)$. "Even when the experts introduced by his own counsel were examining him, and when, had he been intelligent he should have known that it was to his advantage to make the best possible appearance, to give them every possible help, yet when his dinner was brot into his cell, he ignored the people who had been sent to help him. As one of the experts testified, 'As between soup and safety, Jean preferred soup." "

Whenever a strong emotion is aroused in these defective persons, it is either thru an appeal to some instinctive fear or anger or else it may happen to be connected with something in which the person has had some experience, as for instance the case of the low grade boy who was afraid of the dentist (p. 274).

The foregoing considerations will make it clear why the manifestations of emotion are different in the feebleminded than in the normal and conversely, why an unusual display of certain emotions indicates a lack of modification which, under such conditions, may be of itself indicative of mental defect.

When we come to consider the complex emotions, we must expect, from what has been said about the origin of these emotions, that the feeble-minded will be significantly lacking. Such is in fact the case. The poverty of neurons in the association areas has a marked effect here.

To begin with the highest, one is perhaps not surprised to find no evidence of reverence among the feebleminded. However, there are certain concrete situations that we might expect would arouse at least a reverent attitude. For example, one usually speaks reverently of 
the dead. Even this is lacking in the feeble-minded. We must not however conclude that they are irreverent: they are merely un-reverent-lacking reverence. George is forty years old, mentally 6, his closest friend had just died and after the funeral George remarked with a chuckle, "Well, I want to go home but I don't want to go that way."

Willie, aged forty, mentally 6 said the day after a funeral, "We buried John yesterday," in much the same tone that he would have said, "Yesterday we ploughed that field."

Lil is a feeble-minded woman thirty-five years old, mentality 10, with a large family. A visitor asked, "Have you lost any children?" Lil replied, "Yes, lost three in two weeks. That's going some, ain't it?" The reply well expresses the extent of the emotion produced by the death of three children.

Another feeble-minded woman, whose husband had been killed in a drunken brawl, was asked how much money she got for it. When she said she got nothing, a neighbour, also feeble-minded, remarked in all seriousness, "What's the use of having your husband killed if you don't get no money for him?"

Awe seems quite as lacking as reverence.

Reproach, tho a relatively simple compound of pugnacity and the parental instinct, is hardly found, probably because the tender emotion neutralizes the anger, as it obviously does in many fond parents who are never angry at anything their children do- "they love them so." John, forty-three years old, mentally 7, has "adopted" two of the small helpless children. He dresses and undresses them, feeds them, and carries them 


\section{THE EMOTIONS OF DEFECTIVES}

about. He has seldom been known to show any signs of anger at either child.

Contempt and scorn are hard to differentiate but are both in evidence in some high grade morons. In the imbecile they are noticeably absent.

Patriotism and vengeance, involving as they do a permanent enduring sentiment, can hardly be expected in the feeble-minded. Indeed true patriotism as a mental state is probably lacking in many adults. Many persons act patriotically thru imitaton, but that the feeling is absent is often proved in critical moments. Similarly vengeance probably requires more persistent attention than is withn the capacity of the moron.

Resentment on the other hand being more momentary is more possible and is doubtless experienced by some morons. Just where the feeling passes over into simple anger is difficult to say. But it seems certain that the feeling of resentment is not experienced by the imbecile.

Of the remaining emotions of McDougall's list, fascination is most in evidence, and some degree of it seems to be present farther down the scale than any of the other complex emotions.

Loathing shares the weakness of disgust, that is because the form of disgust is so primitive, the loathing is limited and hard to detect.

Envy, admiration and gratitude likewise are affected by the element of self-abasement which is difficult to evaluate. They are, to a small degree, doubtless within the capacity of the moron and seem to be sometimes manifested in the high grades. The lack of gratitude, however, is often remarked in this type of person.

Pity is another emotion that is so easily simulated that [155] 
there is little or no conclusive evidence for it. Morons often express pity in language, but this may be only repeating words that they have heard from others.

Thus making ample allowances for errors of clinical observation, it is reasonably safe to conclude that the $a$ priori argument holds that because these complex emotions require the activity of more or less elaborate association neurons, the feeble-minded who lack these neurons are for the most part wanting in these emotions. Lloyd Morgan (39) says, relative to the study of animal psychology: "In no case may we interpret an action as the outcome of the exercise of a higher psychical faculty, if it can be interpreted as the outcome of the exercise of one that stands lower in the psychological scale."

If we apply this dictum to the study of the outward expression of emotion in the feeble-minded it is doubtful if we could credit them with any of the complex emotions, since almost if not quite all their affective life can be classified under the primary emotions. Indeed it is somewhat startling to realize how much of the emotional life of the average man comprises greater or less degrees of fear, disgust, wonder, anger, subjection, elation, and tender feeling!

\section{Summary}

1. The seven primary emotions fear, disgust, wonder, anger, subjection, elation and tender emotion, are combined by means of the elaborate association processes into the complex emotions: loathing, fascination, scorn, admiration, envy, reproach, gratitude, pity, vengeance, resentment, patriotism, contempt, awe and reverence.

2. The primary emotions are characteristic of animals and undeveloped humans. 


\section{SUMMARY}

3. The complex emotions are dependent upon the development of the great association areas and upon the sympathetic system.

4. Undeveloped mind experiences the complex emotions to only a slight degree and some-such as awe and reverence-not at all.

5. Normal adults modify the emotions (1) by reacting to new or different stimuli and (2) by reacting in a different way from the primitive one.

6. Defectives modify very little, hence they are more primitive.

7. Experience, thru the law of association, builds up elaborate neuron patterns, so that a single word, sound, sight or other sensation may set off an elaborate mechanism and give rise to a violent emotion.

8. The emotional life of defectives and to a large extent of even dull normals can be readily referred to the primary emotions.

9. Only higher intelligences have the highest emotions. 


\section{CHAPTER IX}

\section{THOT}

Do Animals Think? Some will answer, it depends upon the animal. Some will say, it depends upon the definition of thot. Does the hen think when she lays an egg? Does the bird think when she flies away at the sound of a gun? Does the new-born chick think when it pecks at the bright spot? Does the dog think when he gulps down a piece of meat that he finds? Does the man think when he winds his watch at night? Does the amoeba think when it surrounds a food particle? Is there a thot connected with the knee-jerk? Does a bird think when it builds its nest? Does a carpenter think when he drives a nail? Does a man think when he gets his finger into a gas flame and draws it away suddenly? Most of these questions the reader has answered promptly in the negative. To some of them he has given the answer, it depends upon the definition of thot. And finally, a few readers will have answered some of the questions in the affirmative.

Practically every one agrees that the lower forms of animal life do not think, and certainly every one believes that normally developed adult human beings do think. Where between these two extremes is the line to be drawn?-if it can be drawn. Thomas Burnet said, "No man thinks but he is conscious that he thinks." Moreover, all would agree that there is no thot connected with the kneejerk, or with other similar actions, because

[158] 
they are at once classified as reflexes. They take place even when the brain is severed from the body; and by common consent the brain is necessary to thought. Most people would agree also that the bird does not think when it flies away at the sound of a loud noise, or when it is building a nest, because these are instinctive actions. This brings us nearer to our goal, but we may go even further.

It is conceded, by psychologists at least, that a man does not necessarily think when he winds his watch, since it is well known that he may wind his watch while thinking intently about something else; and often when the act is completed he does not know what he has done. Moreover, a very common explanation of why one does many things that were not desirable or advantageous is that he "did not think," which is undoubtedly in many instances literally true. We have now ruled out of our early concept of thot all reflex action, all instinctive action, and a good many other actions, which we commonly explain as being due to habit, or to what the psychologist calls automatic action.

These actions all agree in at least the one point that the action itself takes place immediately upon the application of the stimulus. If now we turn to our nerve pattern concepts we can see that in all these cases there is a definite, ready prepared neuron pattern and an uninterrupted flow of nerve energy from the first application of the stimulus to the final muscular activity. We may therefore conclude, at least provisionally, that under such circumstances of nerve action there is no thot.

But suppose the man in winding his watch finds that he cannot turn the stem, or that it turns without the usual resistance or clicking that means that the spring is being 
wound up; at once a definite state of consciousness is aroused and he says, "What is the matter?" He is now thinking. Suppose the man who would draw his hand away from the gas flame finds, before he gets it fully away, that something impedes his action and he cannot withdraw his hand far enough to entirely escape the flame. He looks around to see what is the matter and finds some other way of escape. He has evidently been thinking. Suppose the meat which the dog would eat is in a box with a glass cover and when he attempts to grab it he strikes his nose against the glass. If he should examine the situation, discover that the glass cover was hinged and proceed to raise it up and get the meat, or if he should step aside and pick up a stick or a stone and break the glass and get the meat, we should be very apt to conclude that he was thinking.

In these and similar actions there is again a common element, there is an interference with the normal completion of the action. The flow of neurokyme which, started by the stimulus, would lead thru a simple neuron pattern to a proper action, has been interrupted or impeded and the action cannot be performed. Again we may conclude provisionally that thot results when the action which would naturally follow from the stimulus is impeded, prevented, or delayed; in other words, when the natural path of the nerve energy is temporarily blocked and the usual action does not follow promptly. This conclusion, however, is not quite safe; because the dog who thrusts his nose against the glass, thus being prevented from getting the meat and swallowing it, would not necessarily make any further attempt to get it. The natural flow of his nerve energy is impeded but he simply stops. We would not conclude that he had thot. Even the man 
winding his watch might be conscious that he had not attained the end-the stimulus had not led to the usual result, but even so, he might put the watch back in his pocket still without thinking.

Evidently the mere interruption of the flow of the nerve energy does not make thinking a necessary consequence. Let us then examine further. The man who finds that his watch does not wind may very probably say, "Oh! a mainspring broken." This we understand from our previous study means that the neurokyme has now flowed across into an associated neuron pattern-a neuron pattern established by previous experience. That neuron pattern was complete in itself and led to its appropriate action. Now that the nerve energy started by our original stimulus, watch winding, has found its way into this other pattern, it also attains its end and the circuit is complete.

We made another assumption, however, that the man might have put the watch back into his pocket, balked in his effort to wind it, but doing nothing. In such a case it is easy to conclude that the nerve energy which had been started by the original stimulus is simply dissipated, as happens when a stimulus is applied for which there is no pattern or no association. Also, in the case of the dog, he would most likely not find a way to open the box, or to break the glass, but would rub his nose against the glass until something else attracted him and a new stimulus led to another line of action-again the original energy is dissipated.

The feeble-minded regularly show this dissipation of mental energy-this failure to make a useful association. Eddie is a twenty-year old imbecile of mentality 6 . He has been trained to scrub floors. Every week he 
scrubbed an unvarnished floor in the laboratory. He started in the corner where he had been told to start, washed and wiped what he could reach; moved over, washed and wiped the next section; and so on. He could easily see where he had just washed and properly joined on the next section. After a time the floor was varnished and then Eddie was told to wash it up in the same way, using now only cold water without soap. Because the water did not change the colour of the varnished floor, Eddie could no longer tell where he had washed and where not; consequently he could never learn to wash the floor. He would either stay in the same place, washing and wiping it over and over again, or he would wander around at random, washing here a spot and there a spot as the fancy took him. His motions were automatic or impulsive; he used no thot to discover a way to tell what part he had washed and what part not.

Tom is a talkative imbecile thirty-four years old, mentality 6. He will tell you with great enthusiasm that he had a perfectly splendid dinner, but when asked what he had for dinner he becomes speechless. He is unable to think of anything that was on the table. Altho the writer has talked with him almost daily for a dozen years, when he recently playfully pretended not to know him, Tom was thoroly puzzled and speechless. After a faint, "You know me, I am Tom," he turned and left the room, unable alike to think that it was a joke or of any further way of proving his identity.

Bert is a high grade imbecile, twenty-seven years old, mentality 7 , who always has a way out that is satisfactory to him, but it is automatic and not thotful. Asked on Lincoln's birthday, "Whose birthday is it today?" he answered, "Lincoln's." "How do you know 


\section{THOT}

it is Lincoln's?" "Because the flag is flying." "Who was Lincoln?" (Emphatically) "Washington." "How often does his birthday come?" "Oh, I don't know. Perhaps once every two years." Bert picks up scraps of conversation and strings them together without any thot. He heard some one talk about Vernon Castle's accident and came in with the announcement. "Vernon Castle fell four feet out of an aeroplane and was killed." From which he concluded that as for him he "would stay on the earth or under the earth, but not up in the air."

Garry, a high grade moron, twenty years old, mentality about 10, writes to the Superintendent: "Dear Professor :-Am sorry to say that I am getting my mind up to run away again."

Two high grade morons who had run away were accidentally met by the superintendent of the institution in the railroad station in the city. It would have been perfectly easy for them to escape, but they could think of no way out of the difficulty and passively followed him back to the institution. Evidently the blocking of the neurokyme is not of itself indicative of thot.

If our assumptions are correct we at least have a clue, since it is possible to see a plausible explanation in the view that when the neurokyme is blocked in its natural course it flows into some other ready made pattern, if any such pattern is available; that is to say, if the person has had experiences in any way associated with the present one. The man could not say, "Broken mainspring," unless sometime he had had the experience of a broken mainspring.

Putting all this together we should have to conclude that thot appears when the neurokyme in a simple neuron 
pattern is interrupted under conditions where there are associated neuron patterns into which that nerve energy may flow and lead to action. Two facts will help make this conclusion plausible; first our hypothesis assumes the presence of neuron patterns, the result of associated experiences. This fits well with what we know of thot. It is the person with large experience that is able to think out a problem, while poverty of thot and poverty of experience go together.

The second point is that our view is thoroly consistent with the history of those actions which are neither reflex nor instinctive and yet do not involve thot. Take the watch-winding itself. It is certainly not instinctive, and in the beginning, the first time one winds a watch, it does require thot and illustrates all that we have said. But once the new path is established and the nerve energy finds a way out so that the stimulus, the sight of the watch, leads to the act of winding, there is no longer an interruption of the flow of the neurokyme and it becomes automatic.

Now that we have a picture of the thot process itself, we may discuss some terms in common use and usually considered to be psychological topics. These are perception, judgment and reasoning.

We have just seen that when the simple flow of the neurokyme from the stimulus to the muscular activity is interrupted we have thot, provided the neurokyme finds another pathway leading to muscular activity and is not entirely dissipated. It will be evident that there are varying degrees of elaborateness in the neuron patterns that are substituted for the original ones and that on this basis it is possible to classify or discuss different degrees of thot. 


\section{PURE SENSATION}

We have already used the term sensation and contrasted it with idea, but we have not as yet defined it. Sensation is the consciousness of a stimulus-the consciousness that results from the flow of neurokyme, which, started by the stimulus in the sensory neuron and being conveyed to the cortex, passes at once, by way of an association neuron, to a motor neuron and out to the muscle. Thus it is the simplest kind of cortical neuron pattern.

It is often stated that adult human beings seldom have pure sensation. This is because experience has built up so many associations with every kind of stimulus that the neurokyme started by the stimulus does not stop with the simple pattern but goes on to a more elaborate one which makes the situation mean something. A loud noise, a bright light, a taste or an odour is no longer a sensation to us because it is immediately the sound of a gun, an electric light, the taste of bitter, the odour of illuminating gas. That is to say, each one is something more than pure sensation.

Even when we recognize the stimulus as a sound, $a$ light, a taste, or a smell, we have gone beyond the limit of pure sensation. We probably come the nearest to a pure sensation in the case of certain vague and indefinite pains, for these are unnamed and often indefinitely located and seem to be nothing but, as we describe them, sensation of pain. A blow on the back of the head which results in "seeing stars" is perhaps nearly pure sensation of brightness. For since it does not come thru the eye we do not think of it as being an eye sensation of colour or brightness, and one so seldom has the experience that it has not become associated even with its cause. Even in these cases, however, the neurokyme does flow 


\section{PSYCHOLOGY OF THE NORMAL AND SUBNORMAL}

into another nerve pattern and we have something more than pure sensation. This is inevitable since the pure sensation can have no meaning for us unless it is associated with something else. With this attempt to indicate what a pure sensation is, we may pass on to the next step; and here we have simply to follow the neurokyme aroused by the stimulus into the nearest neuron pattern.

When rather strong air vibrations which we call sound strike the ear of the infant, he turns his head in the direction of the sound. This is probably a pure reflex, since the turning of the head is not the result of any thot or judgment, plan or determination. Yet the consequences are of great importance. For when he turns his head, rays of light stimulate his retina and arouse another simple neuron pattern in the brain. This, like the other, taken by itself might be a simple reflex and arouse no consciousness. But the two taken together are associated as we have already explained and we have a sound-sight association.

Even here it is difficult for us not to pass over a large amount of experience and say, he now sees what caused the sound; but we must try to realize that in the first instance there is no such conclusion. The sight, the sound, simply exist for him together,- the one- the other. It is true as we have already explained that the next time this sound stimulus strikes his ear he will "think of" the stimulus that formerly aroused his visual area. Or if he sees the object that aroused the visual area, he will "think of" the sound that aroused the auditory area on the previous occasion.

Thus the two simple neuron patterns, each of which by itself would have no meaning, now come together to 


\section{PERCEPTION}

form a larger neuron pattern and we have the first elements of thot. Until language appears there is nothing else. These two sensations are associated and either one suggests the other. The psychological name for this process is perception. It is also called judgment.

The higher grades of feeble-minded make this connection comparatively easily, but as we go down the scale to the middle and low grades the association is made with increasing difficulty.

On three successive occasions, Kirk, a middle grade imbecile, deliberately put his hand on a hot radiator and held it there until it was burned. Evidently he was unable to make any association between the familiar radiator and the burned hand. Even with the higher grades of children it is often necessary to point out the connection definitely and consciously in order to make an association which is necessary for their comfort or well-being.

Unfortunately for our psychology, we adults can never know just how the primitive associations come together and have meaning for us, in other words, how a perception is formed. But we do have a description of the process by one in whom, on account of blindness and deafness, the process was delayed so that she remembers it. Helen Keller (29) has given us a graphic description of the way in which it dawned upon her mind that things have names. It must be remembered that this is not quite the way it comes to the infant, because Helen Keller had begun to talk a little before the illness had left her blind and deaf and she herself says, "Suddenly I felt a mystic consciousness as of something forgotten-a thrill of returning thot." Following is her wonderful description of this process. 
"The morning after my teacher came she led me into her room and gave me a doll. The little blind children at the Perkins Institution had sent it and Laura Bridgman had dressed it; but I did not know this until afterward. When I had played with it a little while, Miss Sullivan slowly spelled into my hand the word "d-o-l-l." I was at once interested in the finger play and tried to imitate it. When I finally succeeded in making the letters correctly I was flushed with childish pleasure and pride. Running downstairs to my mother I held up my hand and made the letters for doll. I did not know that I was spelling a word or even that words existed; I was simply making my fingers go in monkey-like imitation. In the days that followed I learned to spell in this uncomprehending way a great many words, among them pin, hat, cup, and a few verbs like sit, stand and walk. But my teacher had been with me several weeks before I understood that everything has a name.

"One day, while I was playing with my new doll, Miss Sullivan put my big rag doll into my lap also, spelled ' $\mathrm{d}-\mathrm{o}-\mathrm{l}-\mathrm{l}$ ' and tried to make me understand that 'd-o-l-l' applied to both. Earlier in the day we had had a tussle over the words ' $\mathrm{m}-\mathrm{u}$-g' and 'w-a-t-e-r.' Miss Sullivan had tried to impress it upon me that ' $\mathrm{m}$-u-g' is $m u g$ and that 'w-a-t-e-r' is water, but I persisted in confounding the two. In despair she had dropped the subject for the time, only to renew it at the first opportunity. I had become impatient at her repeated attempts and, seizing the new doll, I dashed it upon the floor. I was keenly delighted when I felt the fragments of the broken doll at my feet. Neither sorrow nor regret followed my passionate outburst. I had not loved the doll. In the still, dark world in which I lived there was no strong senti- 


\section{HELEN KELLER QUOTED}

ment or tenderness. I felt my teacher sweep the fragments to one side of the hearth, and I had a sense of satisfaction that the cause of my discomfort was removed. She brought me my hat, and I knew I was going out into the warm sunshine. This thot, if a word. less sensation may be called a thot, made me hop and skip with pleasure.

"We walked down the path to the well-house, attracted by the fragrance of the honeysuckle with which it was covered. Some one was drawing water and my teacher placed my hand under the spout. As the cool stream gushed over one hand she spelled into the other the word water, first slowly, then rapidly. I stood still, my whole attention fixed upon the motions of her fingers. Suddenly I felt a misty consciousness as of something forgotten-a thrill of returning thot; and somehow the mystery of language was revealed to me. I knew then that 'w-a-t-e-r' meant the wonderful cool something that was flowing over my hand. That living word awakened my soul, gave it light, hope, joy, set it free! There were barriers still, it is true, but barriers that could in time be swept away.

"I left the well-house eager to learn. Everything had a name, and each name gave birth to a new thot. As we returned to the house every object which I touched seemed to quiver with life. That was because I saw everything with the strange, new sight that had come to me. On entering the door I remembered the doll I had broken. I felt my way to the hearth and picked up the pieces. I tried vainly to put them together. Then my eyes filled with tears; for I realized what I had done, and for the first time I felt repentance and sorrow.

"I learned a great many new words that day. I do not 


\section{PSYCHOLOGY OF THE NORMAL AND SUBNORMAL}

remember what they all were; but I do know that mother, father, sister, teacher were among them-words that were to make the world blossom for me, 'like Aaron's rod, with flowers.' It would have been difficult to find a happier child than I was as I lay in my crib at the close of that eventful day and lived over the joys it had brot me, and for the first time longed for a new day to come."

It is worth while also to quote her teacher, Miss Sullivan, on the same event. Miss Sullivan's letter:

“April 5, 1887.

"I must write you a line this morning because something very important has happened. Helen has taken the second great step in her education. She has learned that everything has a name, and that the manual alphabet is the key to everything she wants to know.

' 'In a previous letter I think I wrote you that 'mug' and 'milk' had given Helen more trouble than all the rest. She confused the nouns with the verb 'drink.' She didn't know the word for 'drink' but went through the pantomime of drinking whenever she spelled 'mug' or 'milk.' This morning, while she was washing, she wanted to know the name for 'water.' When she wants to know the name of anything, she points to it and pats my hand. I spelled 'w-a-t-e-r' and thought no more about it until after breakfast. Then it occurred to me that with the help of this new word I might succeed in straightening out the 'mug-milk' difficulty. We went out to the pump-house, and I made Helen hold her mug under the spout while I pumped. As the cold water gushed forth, filling the mug, I spelled 'w-a-t-e-r' in 


\section{PERCEPTION}

Helen's free hand. The word coming so close upon the sensation of cold water rushing over her hand seemed to startle her. She dropped the mug and stood as one transfixed. A new light came into her face. She spelled 'water' several times. Then she dropped on the ground and asked for its name and pointed to the pump and the trellis, and suddenly turning round she asked for my name. I spelled 'Teacher.' Just then the nurse brought Helen's little sister into the pump-house, and Helen spelled 'baby' and pointed to the nurse. All the way back to the house she was highly excited, and learned the name of every object she touched, so that in a few hours she had added thirty new words to her vocabulary. Here are some of them: Door, open, shut, give, go, come, and a great many more.

"P. S. I didn't finish my letter in time to get it posted last night; so I shall add a line. Helen got up this morning like a radiant fairy. She has flitted from object to object, asking the name of everything and kissing me for very gladness. Last night when I got in bed, she stole into my arms of her own accord and kissed me for the first time, and I thot my heart would burst, so full was it of joy."

This is perception and it is perfectly described in Titchener's definition of perception as "a group of sensations having a meaning for us."

One difficulty still remains and is found even in the foregoing description of Miss Keller's experience. We must remember that in her ease the association was not between the feel of the cold water and the sound of the word water. She tells us that Miss Sullivan spelled the word doll into her hand, but she also said, "I did not know 
that I was spelling a word or even that words existed. I was simply making my fingers go in monkey-like imitation." The associaton that was made in Helen Keller's mind or brain was between the feel of the cold running water and the group of pressure sensations that were made by Miss Sullivan's fingers upon Helen's palm. What actually happened to her at that glad moment was that these two centres in her brain had become so thoroly associated that she at last realized that they belonged together, that that cold flowing substance was always to be called to mind whenever a certain series of pressures were made in her hand, or as we say, that that substance had a name, and then came the further generalization made by her that everything has a name, that another group of pressures on her hand constituted the name of the doll which she had broken. Let us look a little further into this matter of names. It is a confusing point in psychology, and we shall do well if we can clear it up.

\section{Summary}

1. Reflex, instinctive, impulsive and automatic action involve no thot.

2. Even man "rarely thinks" (James) since so much of his activity is to be classified under one or the other of the above heads.

3. There must be an interruption of the natural flow of nerve energy before we can have thot.

4. Yet mere interruption does not produce thot.

5. Thot appears when the neurokyme in a simple neuron pattern is interrupted under conditions where there are associated patterns into which the nerve energy may flow and lead to action.

6. Experience, which develops the more complicated [172] 


\section{PERCEPTION}

neuron patterns, is the condition for thot. Poverty of thot and poverty of experience go together.

7. Perception, judgment and reasoning are different phases of the thot process. 


\section{CHAPTER X}

\section{THOT-Continued}

What are Names?-It has been said that the sole condition for the enormous mental development of man as compared to the lower animals is in his invention of language. Perhaps it would be better to say his extension of language, for even animals have the elements of language. Psychologically considered, language is an association, such as we have described, between common objects, actions, or feelings and certain sounds, visual symbols, or as in the case of Helen Keller, tactual sensations. The great fact that led to the extension of language was its marvellous usefulness. One has only to think of the great difficulty, the enormous waste of time and energy, that we experience in attempting to communicate with a person who does not understand our language, in order to see something of the significance of language for the race.

Let us follow this thot a little further. We have said that animals have the elements of language; that is to say, there are certain sounds or certain sights that are clearly associated in their brains with certain objects or certain actions. Domestic animals come when called because the sounds used in the call are associated in their minds usually with food. If they are always fed by the same person the sight of the person is associated with food and is to them the symbol. Many of these associations are built up in the brain of the animal rather accidentally, but they may also be brot about by training. The extent to which 


\section{DO ANIMALS THINK?}

this is possible has led people to think that animals understand language and think. The question as to whether this belief is true or not brings us back to the statement that we made earlier; it depends upon what we mean by thot and what we mean by language.

If thot is what we have defined it, it is not likely that animals can be properly said to think to any extent worth mentioning, since it is not likely that they have more than one reaction to the same stimulus. If language means having a few auditory and visual stimuli definitely associated with corresponding objects or acts, then it cannot be denied that animals have the rudiments of language. Not only do they in this sense understand certain words and signs that man makes to them, but they have to the same degree their own language. The sentinel crow has one note that seems to say "All is well," and another that says, "It is time to fly away." The cock says "Come here, here is some food," and also, "Be on your guard, danger is near." The cat has a cry of hunger, a cry of anger, a cry of fear and a call to her mate. Many other instances will occur to the reader.

If on the other hand language is thot of as having anything like the practical value that it has for man, then it is a waste of time to discuss the question, for animals have at most only a few symbols, whereas man has developed thousands and hundreds of thousands of combinations.

Turning our attention to the language of man, it is helpful to remember that a large part of his early perceptions consist in what is in reality learning language,that is to say, learning the names of things; or again in establishing associations between the auditory, the visual or the tactual stimulus and the name of the thing that 
gives rise to the stimulus-as when one hears a piano, sees a piano or touches it. The child sees a certain animal and is told it is a horse. It is common to say that he perceives a horse. As a matter of fact he has only learned the name, has formed an association between a certain visual image that he gets from looking at the horse and an auditory sound that comes from the word horse as some one tells him "That is a horse," and also an association with the motor expression as he repeats to himself the word horse.

Some have spoken of this tendency to name things as the naming instinct. The persistence of the naming habit and the satisfaction which seems to go with it would give some colour to the suggestion that it is instinctive, were it not for the fact that it so clearly has to be learned. Altho none of us can remember it, every infant undoubtedly has to go thru a long period similar to the one described by Helen Keller. There is a long period before the fact is appreciated that things have names and probably the joy accompanying this discovery is the main element in perpetuating the process. Just as Helen Keller learned many words in the next few hours and days, so every child who has discovered that everything has a name is interested in learning new names.

The unfortunate part, for the development of knowledge, is that so many persons are satisfied with the mere name, apparently believing that to know the name is to know the thing itself. Of course, no more serious mistake can be made. It is not many years since certain subjects of study in our schools were almost $\epsilon$ tirely a matter of learning names. Take for example botany as it was frequently taught from twenty-five to forty years ago. The whole purpose of the study was to learn the termin- 


\section{NAMES AS SYMBOLS}

ology so that a person could take a plant and find its name. Today many a person is considered a botanist whose only claim to the designation is that he knows the names of the common plants or has a portfolio of dried specimens properly named.

The same is almost as true of mineralogy and to quite a degree of astronomy, and even geography, especially all that part of geography which consists in knowing the name of the city that is on a river with a name or the name of the country that lies west of another country named - or the name of the city that is called the capital of a State with a given name.

Even today there is much of this kind of teaching more or less unconsciously retained in the schools; and the popular interest in knowing the names of plants, birds, animals, stars is strong testimony to the fact that, with a great many people, something of the original joy of finding that everything has a name persists into adult life. All this would be superseded, if the fact was fully appreciated that words are only symbols and are of no value except as one understands the thing symbolized. They are a convenient medium of exchange like paper money; but if there is nothing back of them they are valueless.

Prof. Rugh of the University of California has summed the matter up in a single sentence, "Words do not convey ideas, they arouse experiences." As we have said, words are symbols and as symbols for experiences they are convenient; but without the thing symbolized they are not only useless but dangerous.

Much has been made of words in psychology under the head of verbal-types, verbal-associations, verbal-memory, etc. All of which only means that the word, the symbol for the experience, can be associated, remembered and 
dealt with just as the original experience. It is probable that this subject has been given so much importance in psychology because of the fact that there is a so-called "centre" in the brain for visual word images, another for auditory word images and one for motor word images.

Moreover, clinical experience has proved that injury to any one or all of these centres destroys one or all of these functions. A person whose visual verbal centre is destroyed, loses the ability to read and write. He is said to have word blindness or alexia. One whose auditory verbal centre is destroyed cannot understand spoken words ; and one whose motor verbal centre is destroyed is unable to form words and speech. The former condition is called word deafness and the latter dumbness or aphasia in a specific sense. The term aphasia is also used in a generic sense to cover all the foregoing verbal defects.

The accompanying table from Church \& Peterson (9) gives in condensed form the main facts about the different aphasias.

Judgment.-Returning now to consideration of the thot process, we recall that we said that the process defined as perception was sometimes called judgment. Stout says, "Judgment is the yes-no consciousness. Under it I include every mode of affirmation and denial-everything in the nature of an acknowledgment explicit or implicit of objective existence." Titchener calls judgment the simplest thot process. Both these definitions make it clear that judgment as used by the psychologist includes much more than the popular conception which makes judgment very largely synonymous with reasoning, or the result of reasoning.

It has been said that the difference between what we call judgment and what we call perception is a difference 


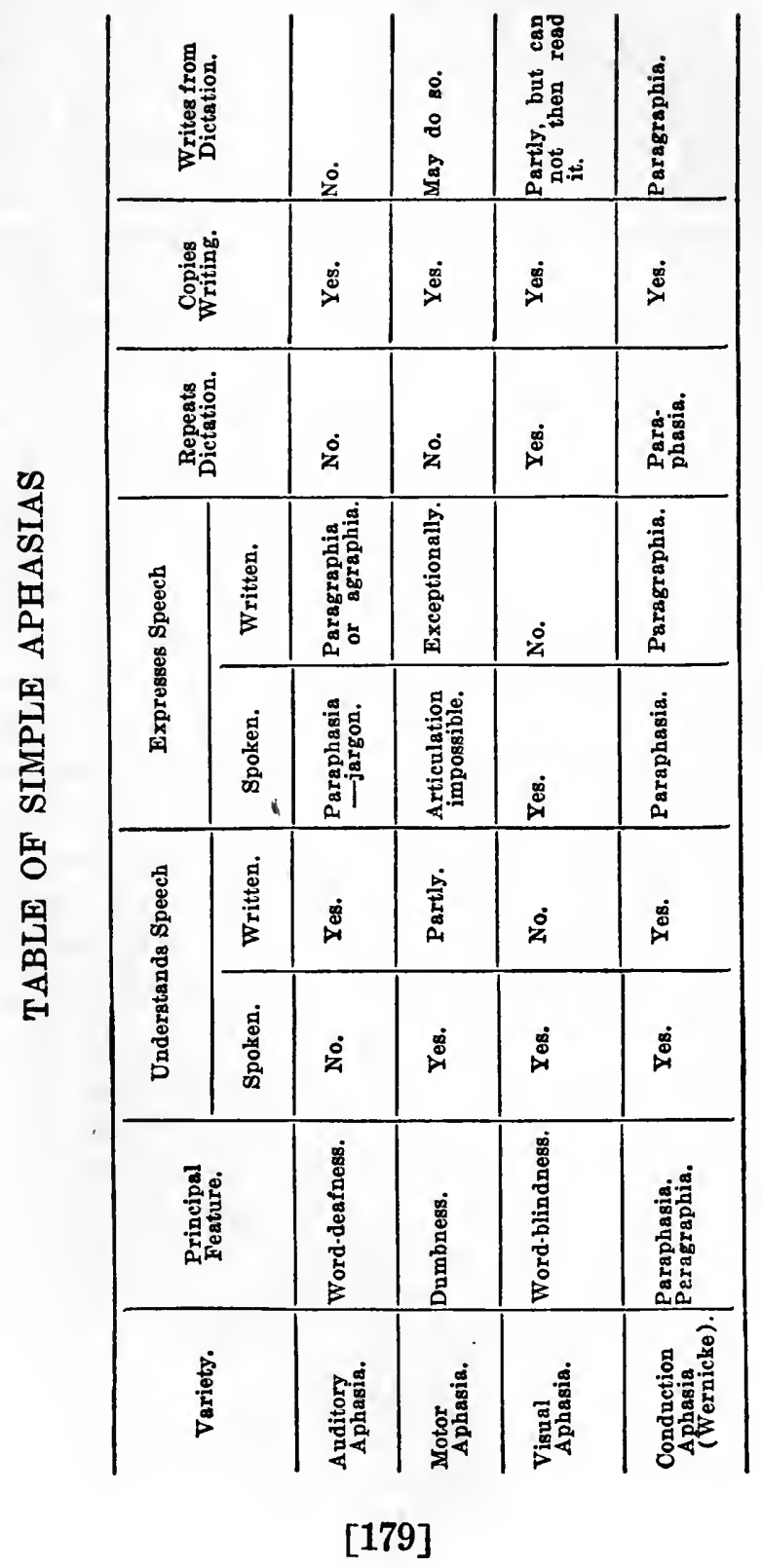




\section{PSYCHOLOGY OF THE NORMAL AND SUBNORMAL}

of degree only. We have said that when two stimuli, each arousing a sensation, become firmly associated, the sensations come to have meaning and this is called perception, or judgment. It is a question whether we should apply the term judgment to this most elemental thot process; whether we should not in fact call this perception and reserve the term judgment for the next higher, more complicated process.

We must now attempt to understand the process itself. Let us take an example. A child gets a stimulation of the retina which gives him a sensation of brightness, called white. $\mathrm{He}$ is also conscious of the wide extent of this whiteness, that it covers the ground. He learns that this white something on the ground is called snow. Passing over certain intermediate processes, let us say that he perceives this object that is called snow-in short he perceives snow. In the brain he has therefore a fairly simple neuron pattern which gives rise to the consciousness "snow." He has previously, and as the result of entirely different experiences, perceived that some things are cold; we say he has a concept of coldness with its underlying neuron pattern. Now it happens that he takes some of the snow into his hand. The neuron pattern underlying the concept "snow" and the neuron pattern underlying the concept "cold" are at once brot together, associated. The two patterns become so closely associated that they form one pattern. The consciousness is also extended to cover what is expressed by snow-cold; or "the snow is cold." This is a judgment.

It will be seen that the only difference between this neuron pattern and the one that gave rise to the first perception is in the elaborateness of the pattern. In the first place there were simple patterns underlying sensa- 


\section{JUDGMENT}

tions. Next there were two patterns each underlying a perception. Now there is one elaborate pattern underlying a judgment. As already stated, perception and judgment really differ only in degree, and yet it seems desirable to make this simple distinction. If we accept this distinction it would follow that just as we said that a perception is a group of sensations, so we could say that a judgment is a group of perceptions.

Moreover we recognize that it is possble to make a great many judgments in regard to one subject, such as: snow melts, snow is frozen water, snow falls in winter. In each one of these judgments we have enlarged our neuron pattern by the addition of smaller patterns gained from experience, until somewhere in the process this pattern becomes so elaborate and the consciousness resulting from its activity is so complex that we cannot hold it all, and some one phase of it becomes stronger than the rest, is the centre of the consciousness, "holds our attention."

It will be seen that, viewed from the standpoint of consciousness, this is all a question of association; moreover, that the original associations making up the perception and the simple associations forming the judgment are associations by contiguity. It is equally clear, however, that many of our judgments are associations by similarity. For example, the child seeing snow for the first time may say, "The ground is covered with sugar." His judgment is based upon an association by smilarity, because snow and sugar are identical in colour.

This makes clear the definition given by Binet (4) who says: "Perception is a synthetic operation resulting from the uniting of information actually furnished by the senses with information furnished by preceding experiences." The information actually "furnished by the 
senses" is the new neuron pattern aroused by the stimulus, which becomes connected with a neuron pattern already formed, that is, "furnished by preceding experiences." Every simple declarative sentence is the expression of a judgment, but it should be noted that it is not necessarily the expression of the judgment of the person who utters the sentence. One may simply repeat the sentence which is the expression of a judgment which some one else has originated. Indeed much of our conversation is precisely that. One does not actually form a judgment unless he has the experience underlying it.

The feeble-minded, having as we have seen good natural memories, often repeat judgments and are given credit for intelligence. This is the explanation of the oft heard paradox "He talks intelligently but he acts foolishly."

Just as perception blends into judgment, so judgment blends into reasoning. It is clear that the illustration of the child who called the snow sugar might just as well have been described as a case of childish reasoning. And this is true, because, as a matter of fact, we are dealing with one and the same process-a proccess of association between neuron patterns which have been built up by experience. The difference is one of degree and not of kind.

Reasoning has sometimes been defined as a train of judgments. It will be clear at once that this will require more experience, more neuron patterns to be associated; and the result of the association is a more elaborate pattern than before. But this is not all. It is not enough to say that reasoning is a train of judgments. The judgments must be associated according to some principle.

There are two kinds of reasoning, inductive and de- 


\section{REASONING}

ductive. Inductive reasoning is reasoning from a particular instance to a general conclusion; it is generalizing. In inductive reasoning we state all we know based on our experiences. A child was given cream to eat. It hap. pened that the cream was a little sour. He reasoned inductively that all cream tasted sour, not liking it he would never taste cream again. Not until he was a man did he discover that cream is usually sweet. He had generalized from the only experience he had.

In deductive reasoning we reason from the general law to a particular case. It is an attempt to decide how to act by referring the particular instance to a class for which we have a general rule. The child in the above illustration had formed the general rule that he did not like cream. Therefore he would never eat anything that was called cream. A is a case of B. In B situations we always act in a particular way, therefore we will act that way in A. In either induction or deduction the validity of our reasoning is dependent upon our experience and the corresponding elaborateness of the neuron pattern.

The inductions of undeveloped mind are often necessarily erroneous, as in the above case of the child and the cream, and often ludicrous. A child sees an animal that is brown and is told that it is a horse; he necessarily concludes that all horses are brown because he has no experience of any other colour. His neuron pattern underlying the concept horse does not include anything but brownness. Not until he has had experience, either directly or vicariously, with horses or other colours will he understand that the generalization that a horse is always brown is not correct, and that his concept of horse must not be limited to brown colour.

Such mistakes are often ludicrous from the standpoint [183] 
of one's own superior knowledge, that is, greater experience; and yet they are phenomena of daily occurrence with the most intelligent people. The story of the Chinese tailor, who was shown a pair of European trousers and was asked to make a pair like them, and who, seeing the patch upon the sample trousers, made the new trousers with a patch, is an amusing one. When, however, the present writer sent to the engraver a chart drawn on paper purposely cross-ruled in blue lines because he did not wish the cross-lines to appear in the finished cut, and the engraver carefully re-drew the cross-lines in black and returned the cut with all the lines in, the situation was not humorous, but annoying. Yet it was a perfectly natural reasoning on the part of the engraver. Such experiences are of daily occurrence and perfectly illustrate the truth that one generalizes from his experience; if the experience is limited, the generalization is quite likely to be false. The feeble-minded are continually making errors of this kind. Jay, eighteen years old, mentally 8 , when asked what a dowel is, replied, "A piece of wood you drill with." In his wand drill at school the wands were long dowel sticks from the carpenter shop. Jay had worked in the shop and knew these sticks as dowels. He generalized from his experience. He had used dowels to drill with therefore dowels are things you drill with. An imbecile or a normal child under nine years of age regularly defines common objects in terms of use-and the particular use that his experience has taught hima fork is to eat with. The scientist surrounds himself with certain rules or precautions so that he may not make faulty generalizations. Even then it is rare that a man publishes a work in which he has made generalizations, that some one does not point out errors. 


\section{DEDUCTIVE REASONING}

Industive reasoning is thus a question of the more or les complete neuron pattern, the result of experience more or less extensive. Deductive reasoning on the other hand is more directly an association between different neuron patterns. Let us examine this statement further. Deductive reasoning is best studied from taking its typical logical form. This is known as a syllogism, which is made up of three parts, the major premise, the minor premise and the conclusion. The following is a classical syllogism:

Major premise-All men are mortal.

Minor premise-Socrates is a man.

Conclusion-Therefore Socrates is mortal.

It will be noted that the major premise is a generalization and has the same liability to error that all generalizations have. A person of limited experience will make some generalizations that are false, then of course, his conclusion is false. The child that saw the snow for the first time really reasoned thus: "All things that look like that are sugar, sugar is nice to eat, therefore, this is nice to eat." The major premise is thus a matter of experience and like all experience is really an association by contiguity.

The minor premise, however, is a case of association by similarity. When we say, Socrates is mortal, we are, in effect, saying he is similar to other men who have died, therefore we substitute, "Socrates" for "all men" in our major premise and come to the conclusion that Socrates is mortal. There is in deductive reasoning then the possibility of error from imperfect generalization in the major premise, or from mistaken ideas of similarity in the minor premise. 
Let us now trace the course of the nerve energy in a case of deductive reasoning.

A four year old child is brought in for a diagnosis of his mentality. The examiner looks at him and says: "He will be an imbecile; he will never have more than 7 year mentality." "Why?" "Because he is of the Mongolian type." Put in syllogistic form this would stand thus :

All children of Mongolian type have a mentality of 7 years or less.

This child is a Mongolian.

Therefore this child will have a mentality of 7 or less.

The Neuron Picture.-The examiner has an elaborate neuron pattern built up from past experience of associations between, on the one hand, visual perceptions of a face with oblique eyes, a head almost as broad as it is long, short stubby fingers, skin that shows poor circulation, weak eyes and sore eye-lids, and the other characteristic appearances of the Mongolian type of defective; and on the other hand, a neuron pattern for the name "Mongolian," for the concepts "imbecile," "mental ages 3 to 7." This, it will be seen, is the neuron basis of our major premise. All Mongols have a mentality of 7 or less. At sight of this particular child the "oblique eyes" starts the above neuron pattern which may or may not be interrupted by a conscious judgment in the form of the minor premise "this child is a Mongolian" (is similar to the type called mongol) and when the pattern is completely aroused there is inevitably the concept "mentality of 7 or less" which we have seen is the conclusion of our syllogism.

As we have stated it, it is impossible to distinguish [186] 


\section{REASONING AND JUDGMENT}

the process from a judgment, the process being stimulus -Mongol-mentally 3 to $\%$. Just when the formal steps of the syllogism will occur, seems to depend on the completeness of the neuron pattern. If the pattern is closely co-ordinated as the result of frequent use, the process will be so nearly simultaneous as to be in the form of a judgment; if, on the other hand, the experience has been so limited that the part of the pattern that gives the outward appearance is loosely connected with the neurons giving the concept "Mongol" and this weakly connected with "mentality 3 to 7 years," the association may be slow and we get the formal steps. It will show in consicousness something as follows: "oblique eyes: stubby fingers : peculiar expression of countenance: looks a little like a Mongolian-yes, he is a Mongolian. But Mongolians are never very bright-mentality usually 4, sometimes 7, never above that: therefore I can assert confidently that this child who is now only four years old will never develop beyond a mentality of $7 . "$

One may even see the process develop still more slowly. Consider that the examiner calls in a student and says:

Examiner: What do you say about this child?

Student: Queer looking boy.

$\mathrm{E}$. Yes, in what way?

S. Eyes are unusual.

E. Yes, what else?

S. Fingers are short; head is queer shape.

E. Well, what type does he look like?

S. Is he a Mongolian?

E. Well, what do you think?

S. He looks like it.

E. Look at him closer. 


\section{PSYCHOLOGY OF THE NORMAL AND SUBNORMAL}

S. (After careful examination.) Yes, I think he is a Mongol.

E. Very well, what does that tell you?

S. Why,-let me see-Mongols are very affectionate and not very trainable.

E. Yes, what mentality?

S. About 4.

E. What is the highest?

S. 7, I think.

E. Can you therefore say that this child of four years is not likely ever to have a mentality above 7 ?

S. Yes.

Here we see the neuron patterns so incompletely associated that the neurokyme flows from one to the other only after continual stimulation by the questions of the examiner. If the student were working alone he would arrive at the conclusion still more slowly and perhaps only after considerable more experience.

If we follow the process in the other direction we may find it so rapid as to be easily considered a perception, and the expert may properly say he perceives a Mongol of not more than 7 year mentality.

Thus again perception, judgment and reasoning are seen to be the same process, differing only in the ease or speed with which the association takes place.

The view of judgment and reasoning set forth in the foregoing pages offers an easy explanation of the defect in these processes seen in the feeble-minded. The most common characterization of a feeble-minded person is "One who is lacking in reasoning or in judgment." Why do they reason so badly? The all embracing answer is because they lack experience. We have seen that experience is fundamental to good judgment and reasoning. 


\section{THE REASONING OF THE FEEBLE-MINDED}

But the question arises, why do they lack experience when they live in the same world as the rest of us? To answer this we must realize that while they do live in the same world, their environment does not mean to them what it means to the intelligent person, because of their weak associations with their resulting incapacity for abstractions and generalizations.

We have seen that in inductive reasoning the great source of error is hasty generalization-generalizing from a single experience. But the normal person quickly makes a generalization from these very facts, that is to say, finding that his generalizations are often incorrect, he forms the concept that it is unsafe to generalize from a few experiences. He therefore refrains and seeks more experiences, either direct or vicarious, that is, if he himself cannot get the experience he asks of others. Even when quite young the normal child learns to do this, and if he sees a zebra for the first time, may ask, "Are they always striped?" The incessant questioning of the normal child gives him information which he uses in his reasoning. His curiosity leads him to explore and get a great deal of direct experience which the feeble-minded child never obtains.

The feeble-minded child is lacking in energy; possibly his instinctive curiosity is weak, altho the lack of energy would probably account for the result. Because of that lack he does not ask so many questions, and he does not explore. This same condition may account for his not seeing the similarity in things and consequently not associating where the normal child does. When he comes to deductive reasoning this error of generalization is fundamental; his major premse is apt to be entirely false. But even if he has a true major premise, his weakness 


\section{PSYCHOLOGY OF THE NORMAL AND SUBNORMAL}

in seeing the similarity of things results in a failure to formulate the minor premise, or he sees similarities that are unimportant and not significant. Consequently his conclusions do not follow.

Theodore, twenty-three years old, mentally 5 , helps in the poultry department. One day he announced that he had found out how to make a hen lay an egg. As a result of his manipulations several hens died. Killing the goose that laid the golden egg is typical feeble-minded reasoning.

A moron woman was helping in the pantry. Several plates of oysters on the shell came back from the table untouched. She promptly threw them into the garbage. Asked why she did it, she replied, "Nobody seemed to want them."

The accompanying picture speaks for itself. The lettering on the wagon was done by the moron who stands beside it. Fig. 44.

Another reason why the mental defective shows poor judgment and reasoning is that his neuron patterns are so simple that he is unable to kesp the whole matter in mind so as to see the connection or relation of the different parts. A low grade imbecile boy if asked whether he is a boy or a girl will say "girl," not because he thinks he is a girl but because he has in mind only the last word said to him and he replies with that. If he were asked if snow was white or black, he would say "black."

Even a high grade moron, when told the following story, "A man walking in the park suddenly stopped, terrified, ran to the nearest police station, and reported that he had seen hanging from the limb of a treewhat?" may answer, "A leaf." He has failed to keep in mind all the conditions; does not see that his answer does 


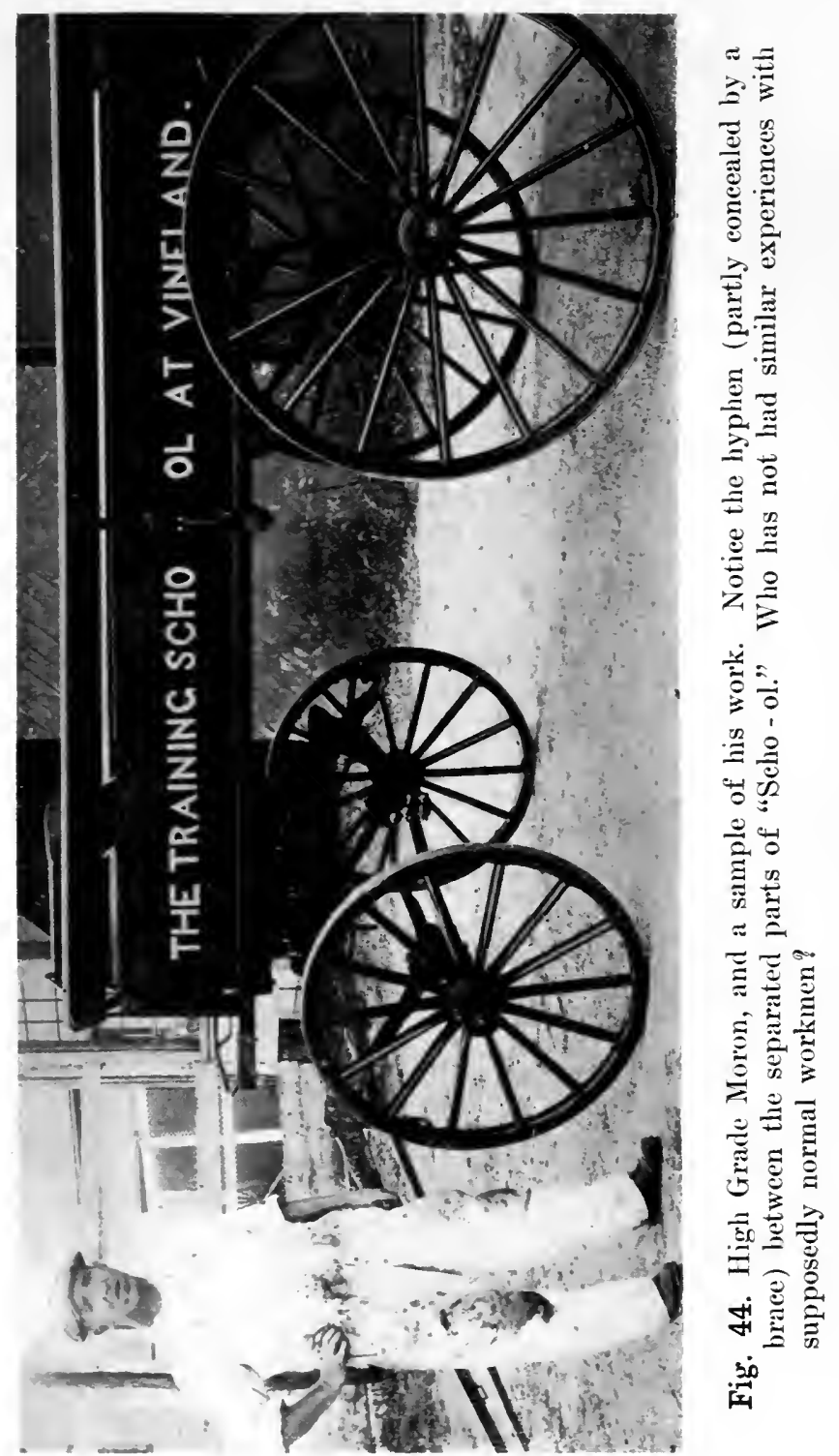





\section{SUMMARY}

not account for the person being terrified or for his going to the police station. He has in mind only the last statement that was made, that "something was hanging from the limb of the tree." A moron under eleven years of age sees nothing absurd if I tell him "I have three brothers; they are John, Henry and myself." $\mathrm{He}$ is not able to hold the whole situation in mind until the incongruity is felt. Charles, a moron of about 8 years' mentality, was milking; the cow switched him with her tail, whereupon he cut off the hairy portion of the tail; and he did this not only to the cow that he was milking, but to every cow in the barn. This was an impulsive action uncontrolled by any reason or judgment.

\section{Summary}

1. Language is an association between objects, actions or feelings and certain sounds, visual symbols or tactual sensations.

2. Animals have a few such associations, hence they have the elements of language.

3. Since animals probably have as a rule but one reaction to each stimulus they cannot properly be said to think.

4. Words, names, are convenient symbols for experiences, but for them to be of value one must have had the experiences.

5. Once the words are established as symbols of experiences, they may be associated, remembered, etc., just as the original experience and with a great saving of nerve energy.

6. Perception is a realized (conscious) relation between two or more sensations, or between a sensation and its word symbol. 
7. Judgment is a realized (conscious) relation between two or more perceptions.

8. Reasoning is a realized (conscious) relation between two or more judgments.

9. Inductive reasoning is a statement of experience-a generalizing. Undeveloped mind generalizes from a single experience-and must, nothing else is possible.

10. Deductive reasoning is substituting, by an association by similarity, a particular experience (or its symbol) for a generalized rule.

11. Perception, judgment and reasoning are different degrees of the same process.

12. Immature mind has difficulty with this process because of deficient association neurons. His difficulty increases with the increasing complexity of the process : he perceives badly, his judgment is worse and his reasoning the poorest of all. 


\section{CHAPTER XI}

\section{ACTION}

THe nervous system with all its wonderful and elaborate mechanism has one function or purpose; viz.: to enable the organism to act. Whether we consider the most primitive nerve structure in the lowest animal in which nerve substance has been found, or the most elaborate brain of the most intellectual man, it is the same. To facilitate action is the end and aim of all nerve structure -of all the processes that go to make up what we call mind. The sensations, the perceptions, the ideas, the attention, the memory, the judgment, the reason, the imagination, all are processes that exist not for their own sake but purely and solely for action. The actions which stimuli produce vary in complexity according to (1) the complexity and (2) the use made of the nervous system. In other words, the perfection of action depends upon the elaborateness of the neuron pattern which in turn is the result of (1) inheritance and (2) the experience of the individual.

The simplest action is the reflex already described, where a stimulus arouses the simplest type of neuron pattern and leads directly to muscular action, without delay or interference. These reflex ares are found thruout the nerve mechanism, especially in the spinal cord and in the sympathetic system. There are great numbers of them and they play a very important part in the vegetative functions-the life processes.

Next is instinctive action, already described and prob[193] 
ably differing in no way from reflex except that it involves a somewhat more extensive neuron pattern, and may be accompanied by consciousness.

Impulsive action or conscious reflex, as it is sometimes called, differs from reflex and instinctive action in that the neuron pattern underlying it is not inherited, but is acquired. When this pattern is once acquired, however, the stimulus leads to its action without any interruption or interference.

Impulsive action is characteristic of childhood and of undeveloped mind but is also common in adult life. If a child, having a stone in his hand, sees a glass window and the thot of breaking the window comes to him, he throws the stone; or it may be it is a penny and he has the impulse to give it to a beggar and he hands it to him. A man, standing on a station platform intending to take a train, suddenly discovers that the train is moving away. He has an impulse to run and jump on the train. Impulsive action has been described as an action following a single idea.

The most complex of all is volitional action, commonly described as action in response to thot, judgment, will. It is preceded by a state of deliberation (literally a weighing of the inducements). It is an action that one deliberately thinks of and decides to do. It is an action that is the final result of a conflict between two or more ideas (or between a perception and an idea). It is a substitute for an impulsive action. The man who has the impulse to run and catch the train will so act if no other thot comes to his mind; but if it occurs to him that he may fall and get killed, then there is a conflict; and it has to be decided which idea he will follow. If the first idea wins he will run after the train, if the second one wins he will 


\section{VOLITIONAL ACTION}

stop. It is a thoroly conscious action, indeed it is characterized by the fact that the attention is held now upon this phase and now upon that.

Viewed from the standpoint of the brain activity, volitional action is the most complex of all actions and gives us the most complex, elaborate and interesting picture of nerve action that can be imagined. As our example would indicate, it is as tho one had two impulses leading to actions that are contradictory and cannot both be performed. Therefore, there is a conflict. Which one will win depends upon several factors. First, it is a question of the elaborateness of the first neuron pattern-the one that would have led to impulsive action if the second idea had not appeared. Second, it depends upon how frequently one has acted in accordance with either one of the ideas. Third, upon how recently he may have acted upon either one. Fourth, it depends upon the emotional content,- - that is, the result and emotional tone of a previous action along the same line. Consider these in turn.

First, the elaborateness of the neuron pattern. This may indeed be so simple that no volitional action is possible, that is to say, it is only impulsive. The child seeing the train start would undoubtedly jump upon it impulsively. He has had no experience that has developed an elaborate neuron pattern such as includes the possible danger. The man's pattern, however, includes, in a vague sort of way, the fact that people are sometimes killed from trying to jump on moving trains. But he has frequently done so and never met with an accident, therefore he makes the attempt. Or it may be that his neuron pattern includes a very elaborate picture of some one falling under a train and being killed. In that case he hesitates and perhaps refrains. But his pattern may, besides [195] 
this vivid picture, include a vivid picture of the necessity of his taking the train. His business will suffer, there is no other way he can get to where he wishes to go; he has promised to arrive on that train and he never breaks his promise - and so on almost without limit.

Frequency of action comes in either for or against his first impulse. If he has frequently jumped on trains and never had an accident, that fact will be a powerful incentive to do the same thing again. If, on the other hand, he has seldom acted in this way but on the contrary has frequently let trains go and leave him, then the probability is strong that he will act the same way now. It may be that without any direct experience himself he has read or known of a great many people who have been injured by attempting to board moving trains. If that phase of the neuron pattern develops, it in turn will be a strong deterrent to his boarding the train.

Recency plays a similar rôle. It may be that he has usually refrained from boarding a moving train, but yesterday he jumped the train with satisfactory result; there will be a strong tendency for him to do the same today. Because the neurokyme has so recently flowed into the pattern leading to that action, it now flows more easily there than elsewhere. On the other hand, he may have frequently boarded moving trains but has very recently learned of some one who had an accident from such procedure; therefore that phase of his nerve pattern, having been recently stimulated, may now determine the result.

Finally the emotional accompaniment to the situation, the pleasantness or unpleasantness accompanying either line of action, will have a strong determining influence in solving the problem. If the overflow of nerve action 


\section{DELIBERATION}

from the sympathetic system is of a pleasant nature, it augments the flow of energy thru the nerve pattern that leads to the action. If, on the other hand, the emotional content relating to either act is unpleasant, we may have an inhibitory action of the sympathetic, whereby the path to that action is blocked. James says one of the most interesting discoveries of physiology was the discovery of nerves of arrest. The pneumogastric nerve, for example, when stimulated arrests the movements of the heart, the splanchnic nerve arrests those of the intestines. That this inhibitive action may overflow to the motor neurons of the central system is quite possible. If so, we have an explanation of the "blocking" of these paths.

A distinction has sometimes been made between deciding to act or not to act and deciding which of two acts to do. Inasmuch as deciding not to act is in reality deciding to continue to do what one had been doing, it all resolves itself to do the same thing psychologically. The process is not different if, instead of there being two alternatives, there are many. The same factors enter into the determination.

It is customary to call the state of mind that one is in before he finally acts in such case a state of deliberation. The term is convenient and without objection except in so far as it seems to imply an ego, an " $I$," that does the deliberating. Much time has been wasted trying to discover what we do when we deliberate, and how we finally choose. While the process in any particular case is enormously complicated and involves such a complex neuron pattern that no one can follow it, yet the general plan is simple.

That happens when we deliberate seems to be that the nerve energy started by the original stimulus is, because 
of the elaborateness of the neuron pattern and the other factors that we have spoken of, delayed in its flow and does not lead promptly to the motor paths which would result in action in one direction or the other.

In other words, in deliberation we are in the very nature of the case inactive until the neurokyme finds its outlet over a motor path in accordance with the conditions that we have described. Since consciousness accompanies the passage of the neurokyme into each part of the pattern, even those that do not lead to action, it is inevitable that we should feel that we are directing the process, rejecting one idea after another until we finally choose the right one - the one that actually leads to the action.

It may contribute to our understanding of how helpless we are, to recall some of those instances where we cannot decide, tho our will to do so is strong. The following from David Harum is typical:

Julius. Which soup should I take?

Mary. I should say the consomme.

Julius. I thought I should like the broth better.

Mary. I don't think it will disagree with you.

Julius. Perhaps I had better have the consomme. Which would you take, Mary?

Mary. I prescribe champagne for you, Julius.

Julius. Don't you think a red wine would be better for me, or perhaps some sauterne? I'm afraid I sha'n't sleep if I drink champagne. In fact, I don't think I had better take any wine. Perhaps some ginger ale or Apollinaris water.

Still better is Mary's description of Julius' struggle with the shoes: 


\section{OVER INHIBITION}

"When I went back to the room I found my brother-inlaw sitting on the edge of the lounge, or what you call it, all dressed but his coat, rubbing his chin between his finger and thumb, and gazing with despairing perplexity at his feet. It seems that my sister had got past all the other dilemmas, but in a moment of inadvertence had left the shoe question to him, with the result that he had put on one russet shoe and one black one, and had laced them up before discovering the discrepancy.

"His first notion was to take off both shoes and begin all over again, and perhaps if he had been allowed to carry it out he would have been all right; but Alice was silly enough to suggest the obvious thing to him-to take off one, and put on the mate to the other-and then the trouble began. First he was in favour of the black shoes as being thicker in the sole, and then he reflected that they hadn't been blackened since coming on board. It seemed to him that the russets were more appropriate anyway, but the blacks were easier to lace. Had I noticed whether the men on board were wearing russet or black as a rule, and did Alice remember whether it was one of the russets or one of the blacks that he was saying the other day pinched his toe? He didn't quite like the looks of a russet shoe with dark trousers, and called us to witness that those he had on were dark; but he thot he remembered that it was the black shoe that pinched him. He supposed he could change his trousers-and so on, and so on, al fine, de capo, ad lib., sticking out first one foot and then the other, lifting them alternately to his knee for scrutiny, appealing now to Alice and now to me, and getting more hopelessly bewildered all the time. It went on that way for, it seemed to me, at least half an hour, and at last I said, 'Oh, come now, Julius, take off [199] 


\section{PSYCHOLOGY OF THE NORMAL AND SUBNORMAL}

the brown shoe-it's too thin, and doesn't go with your dark trousers, and pinches your toe, and none of the men are wearing them-and just put on the other black one, and come along. We're all suffocating for some fresh air, and if you don't get started pretty soon we sha'n't get on deck today.' 'Get on deck!' he said, looking up at me with a puzzled expression, and holding fast to the brown shoe on his knee with both hands, as if he were afraid I would take it away from him by main strength-'get on deck! Why-why-I believe I'd better not go out this morning, don't you?'",

Julius belongs to what James has called the "over inhibited type," his neuron patterns are incomplete because they do not lead to action. It were better that he act wrongly half the time than never to aot at all.

Let us try to diagram Julius' state of mind, assuming that the shoe incident were a part of his preparation for breakfast.

First, hunger arouses the instinct to eat. Had it been possible (food at hand), Julius might have instinctively satisfied his hunger (short circuit A, Fig. 45) but since he must go in search of food, acquired habits prompted him to dress (longer circuit B, Fig. 45). If he had had good habits of action he would have dressed promptly and gone where he could have eaten. But not having fixed habits in relation to dressing, every step was a conscious one. When he came to shoes, for example, he had two kinds, tan and black. Here habit did rule for a time and he automatically laced his shoes without being conscious that he had put on one black and one tan. When he was once conscious of the two alternatives, a decision must be arrived at (Diagram C, Fig. 45). Again 


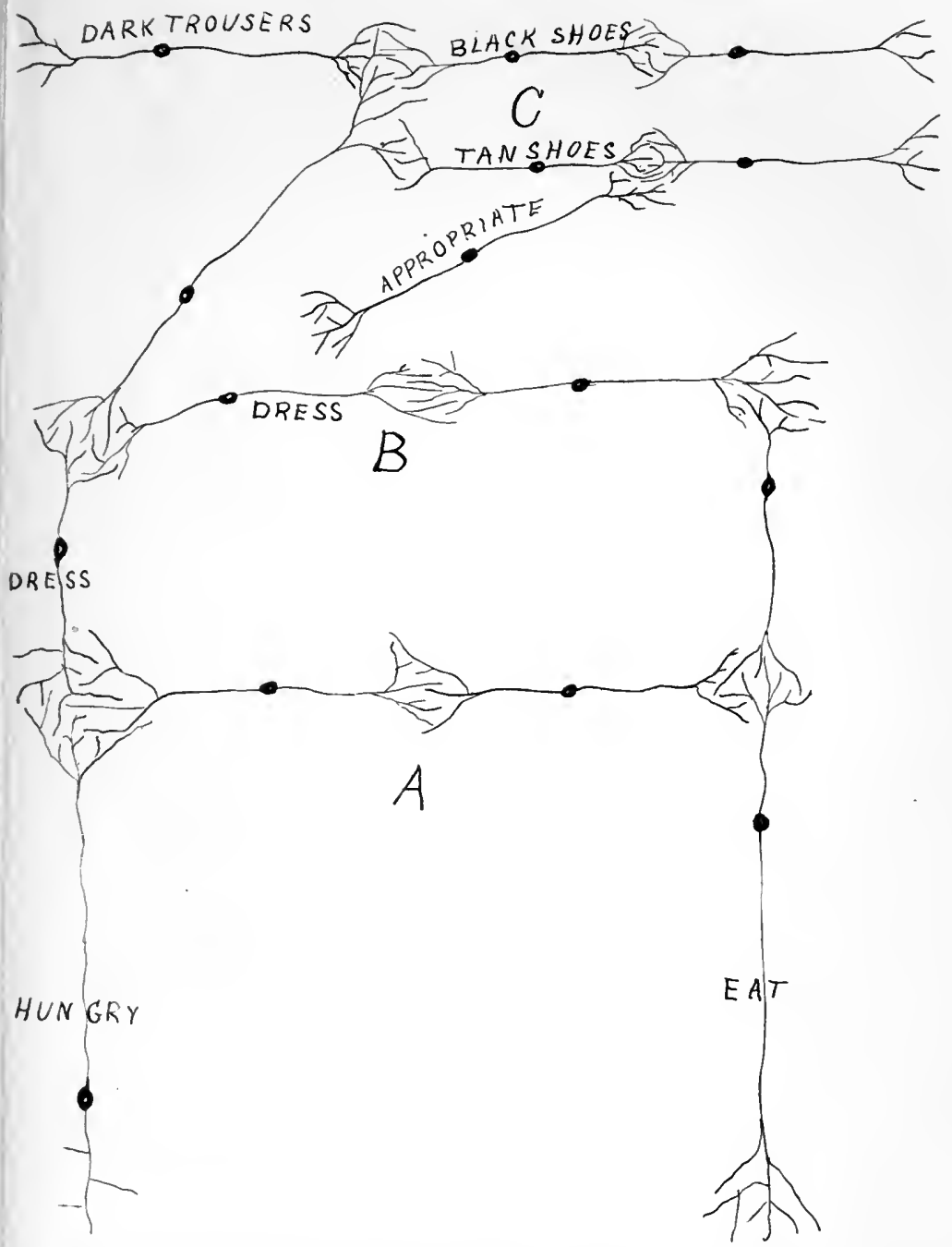

Fig. 45. Scleme to illustrate possible neuron connections in instinctive, automatic and deliberative action. A. Short eireuit instinctive action: Hungry-Eat. B. Longer eireuit automatic action: Hungry-iress-eat. C. Deliberative action: "Tan shoes or black shoes" which? Black shoes pattern reinforced by idea of dark trousers. Tan shoes reinforced by idea that tan are more "appropriate" than the black. No solution is indicated. One or the other would finally connect up with the "dressing" pattern. N.B. A single neuron is taken to symbolize a whole idea or neuron pattern. 

if he had had a fixed habit of wearing black shoes with dark trousers, the neurokyme would have flowed freely in that direction; or had the black shoes pinched his feet so severely that action in that direction was blocked he would have put on the tan. Similarly the other things that came to mind should have re-enforced one path or the other sufficiently to lead to action. But he had a habit of inaction, hence his dilemma was not solved. We can predict that it was solved finally by one of two methods: either (1) some overwhelmingly strong stimulus (argument) forced action in one neuron pattern or in the other; or (2) consciousness was entirely diverted by some extraneous circumstance (wholly new pattern aroused), with the result that when he came back to "shoes" he automatically put on one pair or the other.

Volitional action or will is a matter of the neuron patterns, which in turn is a matter of inheritance plus experience. Prof. James has stated the same thing in different form when he says that "the elements of will are ideas, habit of attention and habit of action." The ideas are the organized consciousness that comes from the neuron pattern. Habit of attention means that the neuron pattern involved in the situation holds the centre of consciousness. Habit of action means that the neurokyme has a definite pathway over which it passes into the motor side, leading to action. The absence or weakness of any of these elements results in lack or weakness of will.

Many familiar facts are intelligible if this view is appreciated. For example, a man is always weak willed, that is, slow to action, in directions where he has no ideas. For example, I find my house has been robbed. I want to 


\section{PSYCHOLOGY OF THE NORMAL AND SUBNORMAL}

catch the thief and secure my property, but the days and weeks and months go by and I do nothing. Why? Because, having no experience in the work of a detective, I have no ideas of how to proceed. The neuron pattern underlying the process of catching the thief has never been developed in my brain. The thot of my stolen goods arouses a very simple pattern about a thief and the possibility of eatching him. If I could see him I would seize him, but that is about the limit of my experience in such matters. Therefore, altho I may be known as a man of action, as strong willed, as full of ingenuity and efficiency and what-not, nevertheless in this particular matter I manifest weak will.

James's expression, that the result is determined by the amount of voluntary attention that one can exert in holding to the right ideas, is of course a question of the associated neuron paths. Voluntary attention is, as we have seen, a matter of neuron patterns formed by experience, so that if one has an idea of acting in a certain direction he can keep that idea in attention only as the result of having a good many associations which will constantly bring up that idea. A man may form a very definite idea of building a fence around his lot. If the neuron pattern underiying the idea of building the fence is connected in his brain with enough neuron patterns so that some one of them will be continually aroused, now by one circumstance and now by another, he will probably be able to keep his attention on the idea of building the fence until it is done, provided he is able to get it done within a reasonable length of time. This latter is an important point. Many times one keeps a certain line of action in mind for considerable period of time, but, if he is baffled in his effort to put in into execution, after

[202] 
a while circumstances of life force other matters into consciousness and this line of action is forgotten.

This brings us naturally to the third clause of James's statement-the "several habits of acting definitely on these ideas to which they have been successfully trained." A man may have a very definite idea about building a fence, he may keep that idea before himself constantly and day after day repeat to himself that he is going to build the fence, but if he is not a man of action, and especially if he is not in the habit of attending to such things as building fences, the probabilities are that he will build no fence and in that particular at least will be weak willed.

This view also solves the difficulty we get into when we attempt to classify ourselves or others as persons of strong will or weak will. If we do not make a snap judgment and decide upon a single experience we are very apt to find ourselves saying, "He generally does what he sets out to do, still there was that matter that he said he was going to do; there was that other matter that he really ought to have done; there is something else he has fallen down on." Can we say he is a man of strong will when there are several things of this kind that he does not accomplish? It will be found that, if we could analyse his consciousness, we would find that in each of those instances he was lacking either in ideas or attention or action.

It is, therefore, impossible to say that one has a "strong will" or "weak will" unless we are speaking in very general terms, which in the end could only mean that, on the one hand, he is a man of ideas and a man of action and usually carries out what occurs to him to do; or, on the other hand, that he has few ideas or so

[203] 


\section{PSYCHOLOGY OF THE NORMAL AND SUBNORMAL}

many interests that he cannot keep his attention upon any one very long, or that he is not in the habit of act. ing, or that sometimes one and sometimes another of these lacks interferes with execution, so that on the whole he is considered a mall who does not often carry out his determination.

There is undoubtedly another element in volitional action which has been perhaps too little considered, that is the emotional content of the experience. We have already stated that it seems highly probable that every stimulus which starts activity in a sensory neuron sends a part of its energy into the sympathetic system, which energy returns more or less re-enforced and adds something which we call an element of feeling to the consciousness that results directly from the stimulus. If the return element is moderate, it gives rise to a mild feeling of pleasure or displeasure; if it is intense, then we have the emotion. This affective element seems to play a real part in the whole question of volitional action. In considering a particular line of action the neuron pattern may be elaborate and complete, one's attention may hold strongly and one may have the habit of action sufficient to perform the volitional act. Indeed one may have performed similar acts many times. But now there comes in the consciousness of unpleasant consequences, or a definite feeling of discomfort. Such affective consciousnass very seriously deters one from carrying out the action.

On the other hand the feeling may be pleasant; even arising to a strong emotion, in which case the action will be performed with unusual and perhaps unnecessary vigour. All the possible degrees and combinations of this influence of the affective state are met with. One 


\section{THE WEAK WILL OF. THE FEEBLE-MINDED}

sometimes sees an angry man, who desires to attack his opponent but is restrained by argument or by force, make repeated efforts to accomplish his purpose, seemingly stimulated thereto each time by a fresh wave of emotion; and one can easily imagine a new flow of energy from the sympathetic system as each new outbreak appears.

We have so far drawn our examples and illustrations from the normal adult, but the one source of data which is most convincing is found in persons of immature mind and particularly the feeble-minded. They are so notoriously lacking in will power that we sometimes reason in the other direction, and when we find a person of exceptionally weak will we are apt to conclude that he is feeble-minded.

The reason for the weak volitional action of the feebleminded will be clear from what has already been said. They lack ideas, that is, elaborate neuron patterns, consequently they lack the power of acquired attention; and they lack action, partly because they are naturally lacking in energy, and partly because their habits of action are few and limited.

One of the facts about morons, so characteristic that it is helpfully diagnostic, is their inability to hold a job.

Every intelligent person knows that there are very few occupations in which one does not at times have to exert all his reason and judgment and will-power to make himself keep at work: The intelligent man keeps at work because he is able to appreciate more or less thoroly the whole situation and to realize that in every occupation there are hardships that have to be borne. The weak minded do not have this foresight-these larger ideasand consequently give up the job upon the slightest provocation. It may be a word of correction from the over- 


\section{PSYCHOLOGY OF THE NORMAL AND SUBNORMAL}

seer; it may be a physical condition which makes the work of the day seem unusually arduous; it may be the attraction of some other job, or any one of scores of things which do not affect the normally intelligent man but will induce the weak minded to leave the work.

This applies not only to the job that one is employed to do, but, with younger persons especially, it applies even to the task in hand. Every teacher or trainer of the backward or defective child knows that if the task is a little long or a little monotonous it requires an immense amount of encouragement to keep the child at it and sometimes even the strongest inducements fail. And the successful teacher knows also that the most successful handling of such cases consists in hitting upon an effective inducement, that is to say, in enlarging the child's stock of ideas and in keeping these ideas in his consciousness until the action is completed. Sometimes a single new thot suggested by the teacher is sufficient to keep the child at his work until it is finished.

It will perhaps occur to the reader that there are many weak minded people who go plodding on, day after day, and year after year at the same job of drudgery with never a complaint and never a suggestion of quitting. It will be found that these are usually cases either of very weak emotion; or of very strong habit, if the job is one that the person has worked at for a long time (as in exceptional cases does occur even with the feebleminded) and his haluit of action keeps him at it. So that in reality these apparent exceptions only prove the correctness of this view. Professor James (28 p. 187) has summed this all up and connects it with what we have already studied by the statement, "To think, in 


\section{short, is the secret of will just as it is the secret of memory."}

An objection is frequently raised to the view that we have presented, that it makes man a slave to his neuron patterns, whereas every one knows that he can determine his own action, that if he acts in one way he knows that he could have acted in the opposite. It is probably very natural that this feeling should arise and yet it is altogether possible that it is a complete delusion. The fact is, that while we have attempted to simplify the process for the sake of a working conception, yet in reality it is so enormously complicated that we inevitably lose ourselves in the mazes; and not being able to follow in detail the workings of this marvellously intricate mechanism of the nervous system we give up in despair and cut the Gordian knot by weakly assuming a mystical something which we call the will as the power which we use in these cases. Thus we think we have solved the problem.

This procedure differs only in degree from that of the uncivilized Indian who could not understand the eclipse of the sun and assumed the presence of a powerful animal that was eating it up. It is not a scientific procedure, we really have no right to expect to understand this matter thoroly because the thing we are trying to understand is the very thing that we understand with. We are in the same difficulty as the poet that cried, "Oh wad some power the giftie gie us to see ourselves as ithers see us." We cannot see ourselves, because it is we ourselves who are trying to do the seeing. The eye cannot see itself. There are, however, other people acting besides ourselves and we ought to be able to understand their action if not our own.

It is an interesting fact that when we look at the action [207] 


\section{PSYCHOLOGY OF THE NORMAL AND SUBNORMAL}

of other people we are much more inclined to admit that they could not act differently than they do. In fact when we do not admit it, it is because we carry over to them our own feeling; we know we can act as we wish, therefore they can do the same. It is true, we do this to a large extent, nevertheless we often do admit that others are the victims of circumstances-the slaves of their enviromment. We are quite accustomed to think of children in this way and especially of the feeble-minded.

Automatic Action. We have already alluded to automatic action and we are now in a position to understand clearly what it is. In discussing volitional action we said that the stimulus which, if unimpeded, would naturally result in an impulsive action, is opposed by an idea which of itself would lead to a different action. We have also seen that in such a case no action is possible until the nerve energy has found an outlet by following the neuron patterns that have already been worked out thru experience. Now, such a pattern once having been followed by the nerve energy will be followed again and the second time naturally more quickly and easily, and still more quickly and easily upon later occasions; so that ultimately this new path becomes as quickly and promptly followed as that of an impulsive action or an instinctive action or even a pure reflex.

Stated in more popular language, this says that once we have decided a question and acted in a certain way, the next time the question comes up we do not deliberate but act as we did before. We do not go thru the labour of deciding it again. Such an action is called automatic. A single illustration will be sufficient. Suppose one lives in a city with the streets laid out at right angles; the Post Office to which he goes daily is, let us say, three 


\section{AUTOMATIC ACTION}

blocks east and four blocks south; there are consequently a large number of different ways that he can get from his home to the Post Office. The distance will be the same by all routes. Several of them are equally interesting and satisfactory. The first time he goes from his house to the Post Office he has to decide which way he will go. But having decided and gone a particular way, the next day he will probably go the same way; and if so, the third day and upon all later occasions he follows that route without the slightest deliberation, without even being conscious of where he has gone and we say commonly and correctly, he has gone automatically.

It may be well to note that at any time something may occur to raise the question again and something may determine him to go by a different route, in which case the action again becomes volitional only again to be reduced to an automatic action. It may also be pointed out that the liability of the question having to be decided a second time depends upon attention, reason, judgment, which in the last analysis is experience-an elaborate neuron pattern.

In automatic action immature mind is sharply differentiated from mature mind. The feeble-minded person, having a habit fixed upon him, changes the habit with the greatest difficulty if at all. The feeble-minded man who had gone to the Post Office by a particular route would continue to go by that route, tho it were flooded and he had to wade thru ice-water. This shows constantly in the training of such a person. Having been trained to do a thing in a particular way, he will continue to do it that way even when conditions are changed and to the intelligent mind the fact is most obvious that it should be done differently. 
This is at once the strength and weakness of the feebleminded. With the limitations that they have, it is extremely fortunate that we can train them to certain lines of action and be sure that once trained they will follow those same lines. On the other hand, circumstances are always liable to occur which make it desirable, and often imperative, that a variation should be made. Therefore it is necessary that such immature minds should be under the oversight of some one who knows their habits, who knows what they will do and who can, so far as pos. sible, see to it that they change their procedure when the circumstances require it.

Automatic action is one of the types of action that constitute habitual actions. We shall therefore consider the subject of habit in the next chapter.

\section{Summary.}

1. The purpose of a nervous system is to enable the organism to act.

2. The perfection of action depends on the elaborateness of the neuron patterns. These depend on the inherited neurons and the acquistions from experience.

3. The inherited neuron patterns are the bases for reflex, instinctive and impulsive action.

4. Volitional action and automatic action depend upon acquired neuron patterns.

5. Volitional action is the result of a conflict between two ideas or between a perception and an idea.

6. Volitional action is preceded by a state of deliberation-the neurokyme interrupted in its natural flow seeks new outlets to motor neurons.

7. The neuron pattern that will be finally followed (end of the deliberation) is determined by the elaborateness 


\section{SUMMARY}

of the patterns involved, by frequency, recency and emotional content-by how much the sympathetic system enters into the process.

8. Consciousness merely makes us aware of what is going on: it has nothing to do with determining the final direction of the flow.

9. Immature mind-including mental defectiveness-is weak in volitional action, because of lack of association neurons or in James' terms, few ideas, weak attention and few habits of action.

10. When the circumstances, giving rise to a volitional action, occur a second time one does not again deliberate but acts as he did before, automatically. This is automatic action. 


\section{CHAPTER XII}

\section{HABIT}

Habit is a term to conjure by. It has been the theme of orators and essayists, lecturers and preachers. James in his famous chapter makes it society's "most precious conservative agent," and for the individual the "spin-" ning our own fates, good or evil, and never to be undone." Rousseau inveighed against it, saying that Emile should be so educated as to "form no habits-except the habit of forming no habits." We excuse our shortcomings by. pleading an unfortunate habit. Too often the emphasis is laid on bad habits and we are urged not to become the slaves of habit.

Much of the confusion has arisen, like Roussean's absurd doctrine, from erroneous conceptions of the nature of habit and its place in human economy. It is true that habit is in a sense opposed to thot; and a person who is too much given over to habit, fails to use his intelligence.

What is this habit that is so much talked about and so little appreciated? Psychologically considered, habit is an automatic or impulsive action, or a group of such actions, so co-ordinated as to result in the performance of a more or less complicated act, with a minimum of consciousness.

Habit has sometimes been called an acquired instinct, just as instinct has been defined as an inherited habit. The only value of these expressions, however, is that they call attention to a certain relation between instinct and habit and probably have arisen because an instinctive 


\section{HABIT}

action and a habit are in outward appearance the same, differing only in origin. The instinct, as we have seen, is the result of activity in an inherited neuron pattern, while habit is more often the result of action in a neuron pattern that is acquired, but which has been stimulated so often that the neurokyme flows easily, and little or no consciousness results.

The natural history of a habit is as follows: First there is an impulse to act in a certain way, fully conscious but unopposed; hence the action occurs; the next time the same impulse comes, action follows with a little less consciousness; soon the action is habitual. For example, in Heidelberg I had an impulse to ride my bicycle along the Hauptstrasse. I did so, very conscious of the narrow crowded street and of the rather strange fact that there were no other bicyclists in sight. Next unorning I repeated it, but thot less about the crowd and the absence of bicyclists. The third morning I thot of nothing but my plans for the day. The habit was formed. The fourth morning I was astonished at being stopped by a policeman. For some time I could not comprehend his gestures or his words. My riding a bicycle on the Hauptstrasse had become so habitual, so unconscious, that it did not occur to me that the policeman referred to that when he said "Verboten." At last however, it was clear! I must not ride on the Hauptstrasse, but could turn down a side street, etc. I did so, fully resolved to obey the law, and did obey it all day. The fifth morning found me a block and a half down the Hauptstrasse and a couple of small boys calling, "Verboten! Verboten!" I promptly dismounted and walked to the next side street. My habit, following that first impulse, had carried me where I had no intention of going. 
The foregoing shows a habit based on an impulse and many habits are of that type. There is, however, another class of habits - those based on a volitional action. Had that impulse to ride along the Hauptstrasse been met by an idea such as, "It is not safe to ride along so crowded a street," the act would at once have become a subject for deliberation and, whatever the outcome, it would have been a volitional action. Had I decided to go ahead and ride down the street, the remaining history would have been the same as it was under the impulse, except that I probably would have more quickly understood the policeman. Having myself deliberated as to the wisdom of riding on that street, more or less elaborate neuron patterns would have been aroused, so that when the policeman stopped me I would almost certainly have had an association by which I would have thot of the bicycle riding and would have been ready for his explanation. It is also possible that the habit would not have been so quickly formed and hence would have been more easily broken on that fourth morning. Having deliberated the first morning, some memory of that would have remained the second and third and later mornings. I would not have lapsed into unconscious habitual action so soonthe fourth morning.

This difference between impulsive habits and voluntary habits is of considerable importance, since the failure to recognize this difference may account for some of the confused ideas and irrational treatment of the subject of habit. For example, had Rousseau said let Emile form no impulsive habits, he would have contributed a valuable suggestion and one which is probably well worth following. Let us see.

Habit, like fire, is a good servant but a bad master. 
Aside from the fact that any impulsive action, being irrational, is dangerous; the fact that it may start a habit, and thus the danger be perpetuated, surely renders the impulsive habit most undesirable.

Moreover, when we classify habits as is popularly done into good and bad, we find that most of the bad habits are of the impulsive kind. An intelligent man neither deliberately starts a bad habit nor keeps it up.

Undesirable habits are either (1) impulsive, or (2) if deliberative, are the result of a deliberation where there was incomplete knowledge, or (3) they are habits that were once useful but have become disadvantageous thru change of circumstances. An example of (2) would be my bicycle riding on the Hauptstrasse, following deliberation. My deliberation resulted in a wrong decision because I did not know that riding on the Hauptstrasse was against the law; an example of (3) is the well-known story of the ex-soldier with his arms full of bundles. When some one shouted "attention," his army habit asserted itself and down went his hands to his side spilling all the bundles. The habit had been deliberately formed and was essential to success in the army, but was very much in the way outside the army.

It is thus evident that impulsive habits are at least dangerous. It would be far wiser to form the habit of not acting impulsively. This brings us to habits that are deliberately formed.

The deliberate forming of useful habits is the most important work of education, both self-education and education by preceptors. It has been too much neglected. It is a matter, as James says, of "making our nervous systems our allies instead of our enemies." It is turning over to our lower nerve centres the performance of 
as much of the routine of life as possible, so that the higher centres may be free to deal with new problems.

Fortunately an immense amount of our daily activity becomes habitual without any effort on our part. But much more benefit could be derived from this power if we consciously planned for it. The business of childhood and youth should be the deliberate formation of useful habits. Compared to this, the mere acquisition of knowledge is a waste of time.

From this standpoint one is compelled to regard the frenzied efforts to make children into precocious scholars as a pitiable perversion of child nature. A nervous system trained to function usefully is the all-important foundation for future greatness. Nervous systems grow and acquire their co-ordinations slowly but surely. To ignore the development of these lower nerve centres and the formation of valuable habits, and instead to waste the time in a futile attempt to hasten the functioning of the great association areas, is nothing less than a perversion of nature. Such efforts invariably prove disastrous in one way or another. Sometimes they result in insanity, sometimes they produce useless prigs, and sometimes early death.

There are gifted children, children who have exceptionally good nervous systems, who have, accidentally or otherwise, exceptionally good early environment and opportunities, and as a consequence are precocious. Such precocity means that the child can, if rightly treated, form an unusually large number of useful nerve habits and perhaps in less than the usual time; and thus be ready with a nervous mechanism remarkably equipped for application to the great problems of life as soon as his great association areas are developed. With such 


\section{HABITS VERSUS NO HABITS}

possibilities it is nothing less than tragedy when parental pride or pedagogical conceit steps in and, ignorant of fundamental necessities, attempts to develop precocious attainments along so-called academic lines.

Nothing can be sadder than the sight of a gifted child exhibiting unusual advancement in reading, writing or arithmetic, but without any well formed habits of eating, sleeping, dressing, playing, working-in short without any evidence of having reduced to the automatic action of his lower nerve centres that great mass of routine activities which must underlie all future greatness.

For the sake of emphasizing this point let us put in parallel columns the activities of the beginning of the day, first for the man who has reduced them to habit, and beside it for the man who has no habits of action in regard to these matters of daily routine.

HABIT

Wake 7 o'clock. Out of bed.

Bath.

Dress in clothes habitually worn on the road.

Breakfast at the club. Cereal, eggs, rolls, coffee.
NO HABIT

(Wake 7 o'clock.) Time to get up. More comfortable here in bed. Have work to do, but there is no rush. Breakfast will get colddon't know that I care for breakfast. However, I will get up.

(Sees bath tub.) Shall I take a bath? Water is pretty cold. I had one yesterday. I like a bath but it takes time. Tomorrow I shall be on the road and have no chance therefore I must take a bath now.

Shall I put on the brown suit or the grey? The brown is prettier but the grey is a little better for travelling because it does not show the dust. The brown is newer but it doesn't fit quite so well as the grey. Think I will wear the grey. Oh, it is at the tailor's!

Let me see, what shall I eat? Shall I take coffee? I like it but 
PSYCHOLOGY OF THE NORMAL AND SUBNORMAL

HABIT

NO HABIT

am not sure whether it is healthy. Dr. Blank says: etc., etc., etc. (Think of the endless possibilities of a Menu Card for a man who has no habits for eating.)

Having now glimpsed something of the importance of habit we come back to our concept of voluntary habits.

Every act that, in the course of the daily life, is likely to be repeated should be consciously considered and a decision reached as to whether it would be desirable as a habit. If the decision is in the negative the action should not be performed, or if it must be done once let it not be repeated, or at least let it never be done without full consciousness and deliberation, keep it always a voluntary action required by the exigencies of the situation. If it be decided that this habit will be a desirable one, then devote all energies to the formation of such habit.

James' (28) maxims for habit forming should be followed to the letter. We repeat them here, but without his masterly comments.

First, in the acquisition of a new habit, or the leaving off of an old one, we must take care to launch ourselves with as strong and decided an initiative as possible.

Second, Never suffer an exception to occur till the new habit is securely rooted in your life.

Third, Seize the very first possible opportunity to act on every resolution you make, and on every emotional prompting you may experience in the direction of the habits you aspire to gain.

Fourth, Don't preach too much to your pupils or abound in good talk in the abstract.

Fifth, Keep the faculty of effort alive in you by a little gratuitous exercise every day.

Just as one should not repeat an act that would be un[218] 
desirable as a habit, so one frequently will repeat an habitual act for no other reason than to keep up the habit. The intelligent man often inconveniences himself rather than run the risk of breaking a useful habit. For example one is in the habit of taking a walk for exercise at a certain hour each day. But there comes a day when business seems to demand that he give up his walk for once. To which he replies, "No, this is an important habit, I will not risk breaking it, by omitting it for a single day." Some rise always at the same hour, summer and winter, Sundays and week days, rather than break the habit. Some insist on eating at precisely the same hours every day-and there is little doubt that such regularity is beneficial to the organism. The organism adapts itself to these regular habits in beneficial ways that are too little appreciated. Stanley Hall used to say that, always having given his academic lectures at 11 A. M., he found he not only lectured better at that hour, but when not lecturing he was more vivacious in conversation at that time of day. Most people who have a regular place as well as a regular time for work, find that they work better in their accustomed place than elsewhere. Undoubtedly this all means a saving of energy ; hence the importance of considering all such matters in the light of habit formation.

We have given three reasons why we have undesirable habits: First, because they were formed impulsively; second, altho deliberative, they were formed on the basis of imperfect knowledge; and third, circumstances have changed so that habits that were once useful are now detrimental. This raises the question of the possibility of breaking a habit. We are often told that habits are easily formed but broken with difficulty. This is one of [219] 


\section{PSYCHOLOGY OF THE NORMAL AND SUBNORMAL}

those half truths that may work much harm thru the potent suggestion that it is almost useless to try to break a well formed habit. We have only to recall our psychology and the nature of habit to see a way out.

It is true that the more firmly a habit is rooted the more difficult it is to uproot. If we have gotten to the condition where the presentation of the stimulus results promptly in a neuron activity that leads to the performance of the act entirely without consciousness, it most certainly will be only by special effort that we shall be able to block this action. But that it can be blocked is a matter of every day experience. In fact, we do change our habits daily. A habit most easily formed is that of sleep. A healthy person can very quickly establish the habit of going to sleep at a certain hour and awakening at a certain hour in the morning. The habit is sometimes so strong that persons will go to sleep at the regular hour no matter what the circumstances, and they will sleep until their usual hour in the morning no matter how much noise or disturbance there may be around them. But let a man change his occupation or his routine of life and, while he may experience some difficulty for a day or two, usually in a surprisingly short period of time he has entirely readjusted his habits in this particular. It is just as easy to rise at six o'clock in the morning as it has been to rise at seven.

Moreover, we change a great many habits unconsciously, as is testified to by the common experience of discovering that we once had the habit of doing so-and-so but we have gotten out of it, we know not how or why. We can see the method best by considering a deeply rooted habit that is really difficult to break. Psychologically the rule for breaking a habit is easily stated. It 


\section{BREAKING BAD HABITS}

is only necessary to restore the consciousness in connection with the action. That is to say, bring it back again to a deliberative voluntary act. Under this new deliberation the new data resulting from experience and the conviction that the habit is bad will result in a decision against the action.

The difficulty, of course, comes from the fact that, once the action has become unconscious, it is performed upon the presentation of the stimulus before we have time to think about it. But here the law of association comos to our rescue, and it is only necessary to call up the stimulus and persistently associate with it the consequences of the undesirable habit so that the next time the stimulus is presented it will arouse the new neuron pattern, which brings to consciousness the undesirable result, and then to act promptly in the new way.

James has given us the key to the situation in his illustration of the man who wished to reform his drink habit and who advertised that he would give any one fifty dollars who should see him in a saloon after a certain date. By this drastic procedure he has associated with the stimulus-the sight of a saloon or the feeling of thirst -the unpleasant consequences-the loss of his $\$ 50.00$. This will inevitably bring to consciousness the whole situation and enable him to voluntarily walk away from the saloon instead of into it.

There will, of course, be a conflict here between the old neuron pattern and the new; and something will depend, as we have already intimated, upon the promptness with which he is able to act upon the new suggestion. If he stands before the saloon door too long he may forget his $\$ 50.00$, in which case he has failed to break his habit. If, however, he walks promptly past, he has won.

[221] 


\section{PSYCHOLOGY OF THE NORMAL AND SUBNORMAL}

It will be seen that in most cases the breaking of an old undesirable habit is best accomplished by the formation of a new and contradictory habit. Therefore James' rules for habit formation are, in reality, the solution of the problem of breaking old habits. In other words, barriers must be built up to prevent action in the direction of the undesirable habit. If I am in the habit of crossing my neighbour's lot on my way to business, but, upon finding that he objects to my passing that way, decide to go around, I may nevertheless find myself half way across the lot before I remember that I had intended to go by. a different way. If, however, upon the next occasion I find a barrier has been built up and I cannot get thru, then I retrace my steps and probably never go that way again. The habit has been broken.

This may suggest a rule for breaking a bad habit. We must build barriers so that we cannot act in the direction of the old habit. It may not be feasible to offer a reward of fifty dollars if we are caught acting upon the habit, but it is always possible to find some way of establishing a barrier which will compel us to reform. We have urged that, so far as possible, habits should all belong to the voluntary class, that we should not act impulsively.

In all this we are thinking of adults who would consciously direct their own habit formation or of normal children whose habits should be moulded by intelligent adults. There seems to be no reason why these facts about habit and the principles about habit formation should not be taught to children fairly early, to the end that they may intelligently co-operate in the establishment of useful habits for themselves.

Let us now turn to the feeble-minded. Here we have [222] 


\section{HABIT AND FEEBLE-MINDEDNESS}

an entirely different problem. We have seen that the feeble-minded are lacking in the higher intellectual processes. It is therefore useless to expect them to deliberate and decide intelligently in regard to the formation of habits.

But on the other hand, because they are lacking in reason and judgment and the ability to deliberate and act rationally in new situations, they are all the more the creatures of habit. It results from both these facts that the wise and intelligent training of the feeble-minded consists largely in establishing in them good habits; and this means of course, not appealing to their reason, which they have not, but in requiring of them such action as will result in good habits. Left to themselves their habits are as apt to be bad as good, but under wise treatment they can be kept from forming the bad habits and made to form useful ones. In this way their lack of judgment and ability to meet new situations is partially counteracted.

A difficulty still exists, of course, in the fact that the feeble-minded person will always act in accordance with his habits, even in those situations where the intelligent person would use judgment and modify his habitual action. There is no way of overcoming this difficulty except by keeping the feeble-minded person in an environment where new situations do not arise; but where, instead, it will always be right for him to act in accordance with the habits that he has acquired. If his lack of reason and initiative prevents him from acting properly in the new situation, it also is a safeguard in that it keeps him from breaking the good habit and forming a bad one.

Among the feeble-minded, habits are unusually persist- 
ent. It must be noted, however, that while the feebleminded man himself is unable to break his good habits and go wrong, it is entirely possible for intelligent persons of evil mind to lead him astray. They can block his good action and establish in him the habit of acting wrongly in accordance with their own suggestions. This points once more to the necessity of keeping these childminds away from the influence of bad persons. The institution or colony managed by good and intelligent adults accomplishes this in the most thoro way. Where such a solution is impracticable there should be in the child's home group some person or persons who would have the responsibility of guiding and protecting him and preventing evil-minded persons from disturbing his well established good habits. When the psychology of habit is thus fully appreciated and applied to all grades of immature mind many social problems will be greatly lessened.

\section{Summary.}

1. Habits are of two kinds, impulsive and volitional.

2. Impulsive habits-resulting from an unimpeded impulse-are, like impulsive action itself, dangerous because they are as likely to be to the disadvantage of the individual as to his benefit. Most bad habits are impulsive.

3. Voluntary habits may be of the highest value. When not useful it is either because the original choice of action was based on imperfect knowledge-resulting in a wrong decision-or because of a change of circumstances.

4. The deliberate formation of useful habits is the most important worl of education.

5. It is not impossible and usually not difficult to break habits, if one goes about it rationally with an understanding of the psychology involved. 


\section{SUMMARY}

6. The feeble-minded form habits easily and change them with difficulty.

7. Therefore habits are of the highest importance in the training, care and control of the defective. 


\section{CHAPTER XIII}

\section{TEMPERAMENT}

In the foregoing discussion of various mental processes or manifestations of mind, we have invariably been driven back to two sources of man's mental make-up: heredity and environment--sometimes called nature and nurture, or as we have repeatedly used the terms, the natural and the acquired.

Under natural endowment we have emphasized the fact that man is born with certain neuron patterns ready for action and that whenever the proper stimulus is applied, the corresponding action takes place. This is necessarily the same in the child as in the parent, the same that has been in the race from the beginning.

Under acquisitions we have referred to the neuron patterns that have been developed thru experience. The acquired neuron patterns are dependent upon the experiences the individual has had, and the way in which different events are coupled up. It is evident that the possibilities here are infinite, limited only by the way things occur in nature or can be brot together.

Up to the present time we have laid the greatest stress upon this matter of experience because it is more possible of control; and being of such profound significance for the individual, it constitutes a problem of education. Since all the possibilities of reason, judgment and will, in the higher forms at least, are dependent upon experience, it should be the function of education to see to it that the child has all of the most desirable experiences. 
It now becomes necessary to consider a little more fully the question of inheritance. It is entirely possible that the variations in inheritance are quite as great as those of environment. In other words, human beings differ enormously in their original nature. It has been customs ary to consider some of these fundamental differences in mental make-up as temperamental.

So little is known of temperament that it is hardly a scientific subject. It is still spoken of as a doctrine. Galen, who flourished in the second century of our era, recognized four temperaments, and the terms are still used in popular language. There were the full-blooded, or sanguine, which is warm, impressional and changeable; the phlegmatic, which is quiet, slow and persistent; the bilious, or choleric, which is energetic and predominantly objective; and the melancholic, which is sentimental and has a marked tendency to subjectivity in excessive form.

It will be noted that these terms are all taken from either real or imaginary fluids of the body, such as the blood, the phlegm, the yellow bile, and the black bile. While the names of the temperaments are still used with the significance above indicated, the connection with body fluids is no longer taken seriously.

Titchener (51) makes temperament dependent upon the rapidity of thot and the strength of the affection, the combination of these giving the four possibilities. For instance, strong affection and quick thot is the choleric, strong affection and slow thot the melancholic; weak affection and quick thot, the sanguine; weak affection and slow thot the phlegmatic.

Recent discoveries make it not impossible that we may ultimately have a theory of tempcrament which will com- 
bine these two views, the ancient and the modern. The modern is reasonable since rapidity of thot would be a question of neurons and neuron conductions, especially in the cortex. While strength of the affection can easily be correlated with Mosso's view which we have accepted, that the emotions are located in or conditioned by the sympathetic nervous system. This view, as a whole then, recognizes the possibility of hereditary differences in the neurons of the cortex and in the structure of the sympathetic nervous system.

Moreover, Cannon's and Crile's discoveries and other work with the ductless glands make it entirely possible that, while we may not be dealing with blood, yellow bile, and whatever fluids the ancients thot of under the name of black bile and phlegm, we may nevertheless be dealing with such fluids as are secreted by adrenal glands, thyroid glands, the thymus, and the other glands of internal secretion. It would seem quite probable then that we are to think of different individuals as having inherited different constitutions in these particulars.

Differences in neurons may be in their chemical composition and the rapidity with which they transmit impulses, the quickness or slowness with which they are restored after being fatigued, their tendency to branching, affecting the number or synapses and probably other conditions little appreciated today.

Differences in the sympathetic system may also be along these same lines. There may be differences in conductivity here, resulting in different infuences upon different glands; and perhaps most variable and important of all, differences in the glands themselves. For example, Cannon's (8) work makes it entirely possible that 
a difference in the influence of a nerve impulse upon the adrenal gland, may make a difference in the amount of secretion poured into the blood, with the result that one person gets violently angry, or is subject to strong fear, whereas another person is moderate in these emotions.

The effect of the glandular secretions upon the whole organism is very definitely known in at least one case, that is, the result of the absence of, or lack of function in, the thyroid gland, giving rise to the very peculiar individual, known as the Cretin.

About all we can do at the present time is to recognize that there are actually these differences in what we call temperament, to attempt to classify individuals, and to note how the presence of the sanguine, the choleric, the phlegmatic, and the melancholic temperaments affects the individuals in their relations to each other and to society.

The problem has not, as yet, been studied in connection with the feeble-minded, altho it is evident that these temperaments are all found among these people and have marked influence upon their social career and bearing; and in that connection are of great importance in the whole question of what is to be the solution of the feebleminded problem. The sanguine and choleric feebleminded persons are the ones who are most apt to get into trouble and be the most serious menace to society; while the phlegmatic and melancholic are much less dangerous.

It should be noted that the whole problem is much complicated by the fact that there is no sharp line between these four temperaments and most persons are more or less a combination of two or three. Evidently the underlying glandular or nervous conditions are unit 
characters which are transmitted in accordance with the Mendelian formula, with the result that we have all possible combinations.

\section{Summary.}

1. Temperament relates to inherited conditions of the nervous system-both cerebrospinal and sympatheticand of the glands of internal secretion.

2. Recent discoveries, notably Cannon's, of the rôle of the glands of internal secretion paves the way for a modern theory of temperament combining the classical one of connection with body fluids and Titchener's modern view of the combinations of quick and slow thot ard strong or weak affection.

3. Temperament undoubtedly plays an important rôle among the feeble-minded determining to a large extent their social adaptation.

4. Roughly speaking, the phlegmatic and melancholic are rather easily controlled while the choleric and sanguine cause most of the trouble met with in their care. 
PART II 


\section{CHAPTER I}

\section{APPLICATIONS}

The test of any theory is the completeness with which it explains known facts. The presentation of the facts of mental processes as set forth in the preceding chapters is in some respects different from that usually found. We have been led to this point of view by the study of the feeble-minded; and have been confirmed in these views by the wide application they seem to have, not only to the feeble-minded but to many common phenomena of life. We shall attempt in the following chapters to point out some of these applications.

Perhaps the most important and far reaching of all the concepts developed is that of mental levels and the arrest of mental development. We have assumed in an earlier chapter that brain and mind develop normally until about the age of twenty, with the average, according to Terman, at about sixteen. The feeble-minded have long been considered cases where development is arrested previous to the point of complete maturity; more specifically, previous to the beginning of adolescence, or as it is more commonly stated, previous to the age of twelve. The discovery of the moron, that is, the recognition of high-grade defectives who differ from normal individuals only in having less intelligence and not by characteristic physical development such as the stigmata of degeneration, led at the same time to the discovery of the border-line cases and then of the dull normal, which leads directly to the concept of arrest of develop- 
ment of all degrees. In other words, the intelligence of human beings ranges all the way from that of the idiot or person of the mentality of a child of 1 or 2 years up to the highest point attained by man-twenty years or more.

This concept once grasped, the desirability of determining the distribution of the different grades of intelligence is evident. The first studies showed the number of persons of relatively low intelligence to be so great as to cast doubt upon the procedure by which the intelligence of various groups was determined.

The result has been a division of students into two camps; the one, following the hypothesis wherever it leads, accepts the conclusion that vast numbers of people are of less intelligence than was supposed, and finds in this fact an explanation of the careers of those whose conduct has long been a problem, those whose inability to get along in the world has been attributed to ignorance, lack of education, accident or misfortune. The other group takes the other horn of the dilemma and denies the hypothesis, saying that the whole view is ridiculous because it makes half the human race little above the moron. Logicians have always claimed that it is not safe to reject any hypothesis because it seems ridiculous, or because the conclusions there from contradict "common sense." We have been led to adopt the first of the two views, not because of a blind acceptance of the hypothesis, but because the conclusion, surprising as it is, and difficult to believe in some cases, on the whole explains the facts of modern civilization more clearly than anything that has been proposed. ${ }^{1}$

1 Since the above was written the use of mental tests in the U. S. Army has established them beyond dispute. 
Let us present the situation as it appeals to us. Fig. 46 is called the curve of distribution. The relative height of the perpendiculars shows the propor-

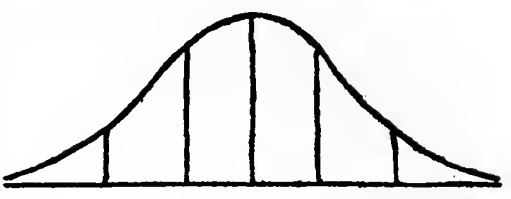

Fig. 46. Curve of distribution. tions of the different degrees of whatever trait is being examined. For example suppose we were considering the stature of human beings. The height of the longest vertical line would represent that stature which is exactly attained by the largest number of human beings,--very nearly what we call the average height. There are almost as many who are just a little taller, a smaller number are much taller, and finally a very few are extremely tall. In the same way to the left are those who are only a little shorter than the average, then those who are considerably shorter, and finally a few who are very short.

Our problem concerns intelligence and applied to this curve of normal distribution it means that there is a certain degree of intelligence which is possessed by more people than any other one degree; approximately what we would call average intelligence. There are almost as many that are just a little higher than the average, there are not so many that are considerably higher, and finally there are a few of extremely high intelligence. On the other hand, there are almost as many that are just a little below the average, there are fewer that are considerably below, and finally there are a few that have extremely little intelligence.

Such, roughly, is the theory of mental levels. Many interesting questions at once arise. Most of them do not concern our present theme. There are two, however, that are of vital importance. First, what is the degree 
of intelligence that will be found at the top of the curve? What is the average intelligence of man? Is it sixteen years as Terman suggests, or is it more or is it less? There is no answer as yet, but the indications are strong that it is much lower than we have supposed. The work now going on in testing the drafted men in the army, will, when published, give us a mass of data that will throw a flood of light on this problem.

If it is ultimately found that the intelligence of the average man is thirteen-instead of sixteen-it will only confirm what some are beginning to suspect; viz., that the average man can manage his affairs with only a moderate degree of prudence, can earn only a very modest living, and is vastly better off when following directions than when trying to plan for himself. In other words it will show that there is a fundamental reason for many of the conditions that we find in human society and further that much of our effort to change conditions is unintelligent because we have not understood the nature of the average man.

It is not necessary here to point out the far-reaching effect of such a discovery-should it prove true. We may, however, allow ourselves one observation. Some may think that this doctrine of mental levels, especially if it leads to such facts as above indicated, is an argument against democracy. It certainly is an argument against certain theories of democracy. Democracy means the people rule (Demos, people; Kratos, ruler). To maintain that mediocre or average intelligence should decide what is best for a group of people in their struggle for existence is manifestly absurd. We need the advice of the highest intelligence of the group, not the average, any more than the lowest. 


\section{DEMOCRACY AND ARISTOCRACY}

Democracy is historically a rebellion against a so called aristocracy (Aristos, best) a rule by divine right, the divine right of kings. The trouble with the old aristocracy is in the answer to the question, "Who decides who is the best?" In the aristocracies of the past a small group of people have said, "We are the best, we have the right to rule." Democracy says: "The entire group must decide who is best, wisest, who can give us the best advice." But will average intelligence select highest intelligence and submit to its rule? It depends upon the character of the highest intelligence, and its attitude toward mediocre and low intelligence. The moron in the community will not select and obey the man who tests high but who uses his intelligence for his own aggrandizement and mistreats those of lower intelligence. But the morons and imbeciles in an institution would select and do obey the superintendent and his helpers because they are working unselfishly to make the morons and imbeciles happy.

Democracy, then, means that the people rule by selecting the wisest, most intelligent and most human to tell them what to do to be happy. Thus Democracy is a method for arriving at a truly benevolent aristocracy. Such a consummation will be reached when the most intelligent learn to apply their intelligence. In other words instead of securing power by such political methods as are now too often resorted to, or by the use of money and "influence," high intelligence must so work for the welfare of the masses as to command their respect and affection.

This is not difficult, once the problem is understood and the right attitude taken. The reason the moron is a menace in society is that he is misunderstood and conse- 


\section{PSYCHOLOGY OF THE NORMAL AND SUBNORMAI,}

quently mistreated. The reason he is a happy, contented, obedient, and useful member of an institution for the feeble-minded, is that he is understood and treated with consideration. His mental level is recognized and every effort made to secure his happiness. The truest democracy is found in an institution for the feeble-minded and it is an aristocracy - a rule by the best.

Another consequence of the discovery that average intelligence is thirteen instead of sixteen is that it prepares us to accept a much larger group of morons, since they are thus brot nearer the apex of the curve and, unless the curve is a very steep one, must be nearly equal in numbers to the average group. The recognition of this fact, if it proves to be a fact, will prepare us to accept the findings of investigations already made and many others being made and to follow, showing that large groups of so-called menials really fall into the moron class. This again enables us to understand their shortcomings, their follies, their blunders and failures. And, what is still more important, it points the way to a wise and satisfactory treatment of these classes by the more intelligent group.

A second question of importance in connection with the doctrine of intelligence levels is: What is the cause of the low intelligence of human beings? Here again there are two views. One group says it is lack of education or training. Therefore, according to them, if the average intelligence is low, it only emphasizes the need of better educational facilities. The other group says that intelligence is something quite different from education; that it is primarily dependent upon the inherited nervous system and neuron patterns; and while education is important and can do much, yet as a rule people display

[238] 


\section{MILLET'S PICTURE}

the intelligence that they do, because of the condition of their neurons; and they behave as they do because of lack of sufficient intelligence to do better.

Every reader of this book knows of a great many people who repeatedly act unintelligently. We are constantly asking, "Why does Blank make such stupid blunders?" "Why doesn't X get along better than he does?" Usually one leaves the question unanswered or else concludes that the person is either uneducated or unfortunate. The hypothesis of mental levels says that as a rule such cases are instances of arrested development of mentality; that they do as they do because they have not enough intelligence to do better. They are not feeble-minded necessarily, but they are more or less below the average intelligence or at least-and what is more likely to be true-so much below the intelligence of the observer that they cannot with any possibility act as wisely as he would in a similar situation. Such a person does as he does because he has not intelligence enough to adapt himself to the complicated environment in which he is placed.

Millet painted a famous picture, known as The Man With the Hoe. Fig. 47. Edwin Markham wrote a poem about it in which he seems to imply that the man Millet painted came to his condition as the result of social conditions which held him down and made him like the clods that he turned over. This is undoubtedly the popular view. The doctrine of mental levels, on the other hand, while not denying that there are men whom circumstances have held down, would lead to the conclusion that the majority of such people as the man with the hoe are where they are because of lack of intelligence. Millet's Man With the Hoe is a man of arrested mental develop- 


\section{PSYCHOLOGY OF THE NORMAL AND SUBNORMAL}

ment-the painting is a perfect picture of an imbecile. Markham's poem thus becomes a pertinent question as to the cause of feeble-mindedness, and how society is solving the problems involved.

We ask the reader to read again the poem with this thot in view.

\section{THE MAN WITH THE HOE}

Bowed by the weight of centuries he leans Upon his hoe and gazes on the ground, The emptiness of ages in his face, And on his back the burden of the world. Who made him dead to rapture and despair, A thing that grieves not and that never hopes, Stolid and stunned, a brother to the ox? Who loosened and let down this brutal jaw?

Whose was the hand that slanted back this brow? Whose breath blew out the light within this brain? Is this the Thing the Lord God made and gave To have dominion over sea and land;

To trace the stars and search the heavens for power;

To feel the passion of Eternity?

Is this the Dream He dreamed who shaped the suns And marked their ways upon the ancient deep? Down all the stretch of Hell to its last gulf There is no shape more terrible than thisMore tongued with censure of the world's blind greedMore filled with signs and portents for the soulMore fraught with danger to the universe.

What gulfs between him and the seraphim! Slave of the wheel of labour, what to him Are Plato and the swing of Pleiades? What the long reaches of the peaks of song, The rift of dawn, the reddening of the rose? 


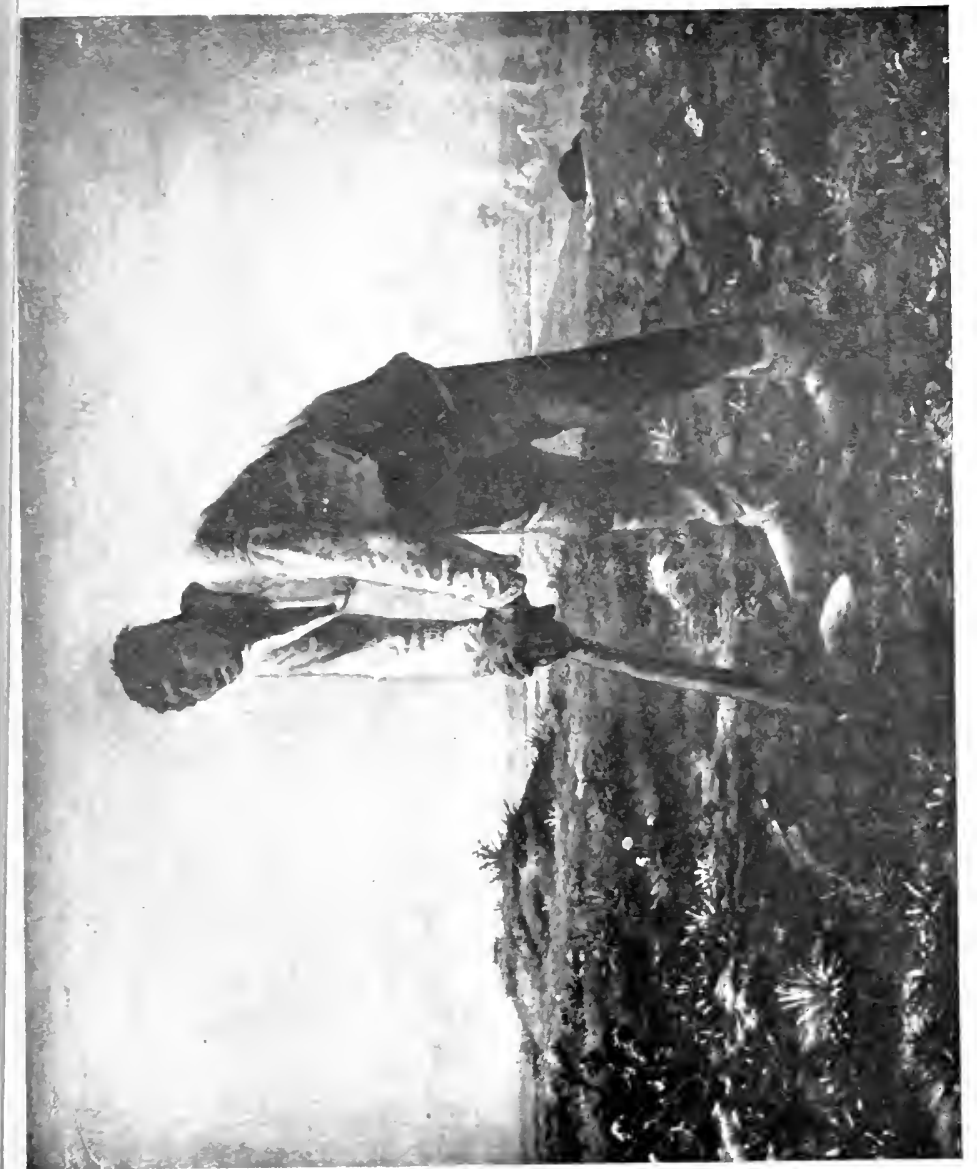

ב

$\Xi$

$\Xi$

$=\frac{E}{\Xi}$

三

$\bar{z}$

$\therefore \equiv$

恶

岮

छ

잉

芯

츤

돈 을

芹导

in

体 



\section{EDWIN MARKHAM}

Through this dread shape the suffering ages look;

Time's tragedy is in that aching stoop;

Through this dread shape humanity betrayed,

Plundered, profaned, and disinherited,

Cries protest to the Judges of the World,

A protest that is also prophecy.

O masters, lords and rulers in all lands,

Is this the handiwork you give to God,

This monstrous thing distorted and soul-quenched?

How will you ever straighten up this shape;

Touch it again with immortality;

Give back the upward looking and the light;

Rebuild in it the music and the dream;

Make right the immemorial infamies,

Perfidious wrongs, immedicable woes?

$\mathrm{O}$ masters, lords and rulers in all lands, How will the Future reckon with this Man? How answer the brute question in that hour When whirlwinds of rebellion shake the world? How will it be with kingdoms and with kingsWith those who shaped him to the thing he isWhen this dumb Terror shall reply to God, After the silence of the centuries?

While, as we have said, the poem seems to imply that environment has made the man what he is, our view makes the cause heredity. But that does not make less pertinent the question "Who made him dead to rapture and despair?" It only pushes back the query to "What is heredity?" Is it the accumulation thru long ages of slight variations downward or is it some sudden mutation or yet again is it a strain of primitive human nature that has never developed? Science has as yet no sure answer. 


\section{PSYCHOLOGY OF THE NORMAL AND SUBNORMAL}

The view that lack of ability to act intelligently is due to lack of education must not be dismissed without one very important fact that bears upon the question. Education as a solution for inefficiency has been tried from time immemorial. Not all, it is true, but many, of those who manifest low intelligence have had the same opportunities, the same school facilities as their more intelligent associates, and yet they have never been able to profit by these opportunities.

Not only have dullards in school had the best efforts of their teachers, but parents have spent their lives and fortunes trying to "educate" these children. Special methods, special schools, special institutions have all tried, and tried with all the zeal that goes with perfect faith. Even institutions for the feeble-minded for years believed that they could improve the mentality of their pupils-all to no avail.

The counter argument is of almost equal force. Hundreds of men, past and present, have displayed high intelligence and yet have had little or no opportunities for education, as education is usually understood. They are called self-educated, or self-made men, which in the last analysis can only mean that, being men of natural intelligence, they have made use of such opportunities as came to them to acquire experience, to develop their neuron patterns; and have actually been able to equal perhaps the highest intelligence known-certainly oftentimes to go far ahead of the average. And that is as true of men living today and functioning in our most complex society, as of men who lived in the past when social conditions were vastly simpler.

Let us turn now to see what the practical acceptance of this view would mean. It means at first, of course, 


\section{RESPONSIBILITY}

a radical change of attitude toward our fellows. It means a complete change of ideas of responsibility. We have learned that the idiot is so little intelligent that he is no more responsible for his actions than an infant. The imbecile of a little higher intelligence has only a very moderate degree of responsibility. The moron has more intelligence, but in the larger affairs of life we no longer hold him responsible. The mere fact that a person is recognized as feeble-minded, whether imbecile or moron, is sufficient to excuse him from punishment for crimes or misdemeanours.

When we pass that imaginary line that separates the feeble-minded from the so-called normal, we come to those who are supposed to be responsible, but who in fact are only responsible to a degree slightly above that of the high grade feeble-minded. This being understood, it at once becomes incumbent upon those who have intelligence, to recognize the limitations of those of arrested development.

Once this condition is appreciated, society must be so organized that these people of limited intelligence shall not be given, or allowed to hold, positions that require more intelligence than they possess. And in the positions that they can fill, they must be treated in accordance with their level of intelligence. A society organized on this basis would be-a perfect society. Because we do not recognize mental levels, we put men in positions involving more responsibility than they are capable of carrying and then trouble arises. We are learning now that there are relatively few things that the moron can do. We are learning that he must be treated as a child.

We must next learn that there are great groups of men, laborers, who are but little above the child, who 


\section{PSYCHOLOGY OF THE NORMAL AND SUBNORMAL}

must be told what to do and shown how to do it; and who, if we would avoid disaster, must not be put into positions where they will have to act upon their own initiative or their own judgment. We must learn that the distribution curve applies to every community. There are only a few leaders, most must be followers.

We have partially recognized the foregoing facts, and in a crude way have attempted to meet the situation. There are certain trades, occupations and professions that no man can enter without a State license. Why? Because it has been discovered that these are positions of great responsibility. Errors of judgment or lack of intelligence may result in great loss of property or of life. A man must be licensed to practise medicine because if he does not have a certain amount of intelligence he may do untold harm to the community. A man must be licensed to run an engine because it has been discovered that an unintelligent engineer may cause enormous loss of life and property. But our methods are as yet too crude and empirical.

The ideal solution of the problem involves two things. First, the knowledge of the amount of intelligence required in any work; and second, the knowledge of the amount of intelligence possessed by the would-be worker in that line. Then it will be relatively easy to fit the individual to his work. We have come to this practice in certain dangerous professions. Human welfare depends upon the right procedure in many cases where danger is not recognized. Efficiency has come to be a great word.

Efficiency comes from having every man fitted to his job, so that no mistakes and no consequent losses come from lack of intelligence. Let us take an iffustration. 


\section{EFFICIENCY}

Suppose two men start into the manufacturing business. The one chooses his employés in what we may call the oldfashioned way, with the result that many men are given jobs that require more intelligence than they possess. It is inevitable that these men will sooner or later make mistakes; will spoil work; will, at a critical moment, do the wrong thing, with resultant loss. All those losses must somehow be covered and the consumer of the product of that factory must, in the long run, pay for them. Another employer selects his men according to some plan, based on a determination of intelligence. $\mathrm{He}$ knows the amount of intelligence required in each job, he ascertains the intelligence of his would-be employés and gives them jobs accordingly. Each man is trained, not simply to do his work, but to do it intelligently. $\mathrm{He}$ meets all the exceptional situations with good judgment. The result is no losses, no accidents. The manufactured product is produced at the lowest cost. That factory is efficient, the other is not. The first man cannot honestly compete with the second. Fortunately some plants are now being run on the new plan. But the great mass of our industries, and much of our social organization today are on the former plan. It is largely an accident if a man gets a job that he is thoroly competent to do. It must be noted that many jobs are filled by men who are competent to manage them when all goes well, but when the unexpected happens, the emergency arises, they break down. We are surrounded on all sides by incompetency, largely because we do not recognize mental levels.

This is a-day of social uplift. Thousands of people have become interested in these social problems and are working to uplift the masses. Many of the efforts-have 


\section{PSYCHOLOGY OF THE NORMAL AND SUBNORMAL}

come to naught, are coming to naught, and will continue to come to naught until this principle of mental levels is recognized.

Many a philanthropically inclined man or society has cleaned up a community or a family only to find that in a few weeks or months conditions were as bad as they were before. One investigation in New York City found one family that had been the recipients of charity from a charity organization society for thirty years; this story could be multiplied many times, probably in every community. What does it mean? That the family has not enough intelligence to profit by the help that is given them. An intelligent man may sometimes be overcome by circumstances and need to be helped on his feet, but once helped to get control of circumstances, he is able to go alone. It is useless to set the unintelligent man up in business and expect him to manage his affairs satisfactorily.

As a rule the drudgery of the world is done by these people of low intelligence, simply because more intelligent people will not do such work. They seek for work where their intelligence counts and the people who are doing the drudgery are, as a rule, in their proper places. This fact should be recognized and they should be helped to keep their proper places, encouraged and made happy, but not promoted to work for which they are incompetent. A certain mother had a feeble-minded boy of moron grade. She secured him employment as a messenger boy. This was well within his capacity. He was of a quiet temperament, obedient and faithful, and did his work well; so well in fact, that the manager was so much pleased that he promoted him to the office. In a 
short time the boy had to be discharged for incompetency and dishonesty. The mother brot him back and this conversation occurred. "Was not my boy a good messenger boy?" "Yes, excellent." "Then will you not employ him as a messenger boy on a task that he can do, and not attempt to make out of him that for which he has not the intelligence?",

We have said that we have learned to recognize the mental levels of the feeble-minded, but we must learn to do the same for a large proportion of the community.

In conclusion, two cautions are necessary. First, the reader must not forget the distinction we have made between education or training and intelligence. In what we have said we do not refer to those persons who undertake a piece of work that requires training, but for which they have had no training. The man who employs a carpenter to build a house may be much more intelligent than the carpenter and yet not be able to do the carpenter's work because it requires training, in addition to a certain degree of intelligence. But it does not necessarily require as much intelligence as the householder possesses. In the institutions for the feebleminded one may find feeble-minded men and women doing tasks that the college man could not perform without training. This only means that the various tasks that have to be performed call for a combination of intelligence and training; and this in all proportions, some requiring little intelligence and much training, others much intelligence and little training. It must not be forgotten either that as a rule the greater the intelligence, the less training it requires to master a particular trade. The second caution is, that it cannot be denied that [247] 
PSYCHOLOGY OF THE NORMAL AND SUBNORMAL

there are people of good intelligence who are incompetent, ne'er-do-wells, eriminals, or other anti-social persons. At present we know little scientifically about this group, yet we cannot deny the existence of such a group. They constitute a real problem in education. 


\section{CHAPTER II}

\section{THE DETERMINATION OF MENTAL LEVELS}

Is Part I, we attempted to show how the various mental processes devolop pari passu with the development of the nervous system. It follows that just as we have stages of growth physically so we have levels of intelligence. In a general way this is not a new thot, since every one knows that a ten year old child is more intelligent than an eight year old, and an adult more intelligent than a youth. It is, however, not appreciated that these levels are as definite as the physical stages; just as a child should be of a certain height at a certain age, so he should have a certain degree or level of intelligence. Nor is it appreciated that this development of intelligence-this growth from one level to the next higher-is inherent in the nature of the growing organism, and is not a matter of education or training. White (55) says:

"Every individual born into the world has, if it could be determined, a definite potentiality for development."

We have also emphasized the fact that a considerable proportion of the human race have not developed to the full capacity or to average mentality, but, on the contrary, have been arrested in various stages or at various levels. In the foregoing chapter we have shown the significance of this arrest in many practical affairs of life. These familiar fasts of differences of intelligence among adults as well as between children of different ages have recently received new significance and great importance from the fact that we have learned to measure 
and determine these mental levels with much accuracy.

It was the great French scientist, Alf red Binet (5 \& 6), that showed us how to measure intelligence, and it was his genius that gave us the first measuring scale. The practical application of the doctrine of mental levels depends upon the ability to measure the intelligence of the individual. If intelligence cannot be measured, there is little practical value in knowing that there are levels of mental development; if it can be measured, then the suggestions of the previous chapter are not only practical but of vital importance.

That intelligence can be measured is no longer in doubt. Aside from the thousands of children that have been tested in the past ten years-and the results confirmedthe present use of mental tests in the United States Army has settled the question. Army officials testify that the psychologist with his measuring scale of intelligence discovers in an hour's time a soldier's ability more accurately and more certainly than the officers can determine it in weeks of observation. The value of this to the army cannot be overestimated. First there is the prompt recognition of high intelligence, with the resultant possibility of having responsible positions filled by competent men; and second, the equally important recognition of those men whose intelligence is so low that they can be trusted only in the most menial positions. How far the actually feeble-minded can be utilized in the army is yet to be worked out. The greatest difficulty here will be that the army officers, having had no experience with the feeble-minded, will not understand how to treat them to get the best out of them. ${ }^{1}$

1 Twelve per cent. (12\%) of the drafted army of the U. S. was found to have too low intelligence to be sent over seas.

[250] 


\section{MENTAL LEVELS}

It is probable that there is plenty of work in the army that requires no more intelligence than that possessed by the least intelligent drafted man. But these low intelligence men must be handled like children if they are to be kept at work at their best. They must be continually encouraged and praised. New work must be as carefully explained to them as to a child. They must not be scolded or punished-in the usual sense. Deprivation of privileges will accomplish all that can be accomplished in the way of correction of mistakes or misdemeanours. Some who have acquired bad habits before entering the army will undoubtedly have to be discharged. Such discharge should, however, be in the form of a commitment to an institution for the feeble-minded. These men, once under the control of the government as they are in the draft, should never again be turned loose in society to live on their own responsibility.

Not only has the use of mental tests in the army settled the question of their value, but we may expect still greater benefits in the form of data that will, first, enable us to extend and perfect the scales themselves; and second, give us a reliable answer to our question discussed in an earlier chapter: what is the average intelligence of man? Present indications point to a level much below our assumed level of 16 years. Whatever this proves to be, it cannot but modify profoundly: our views of the relation of intelligence to citizenship. For example let us assume for the sake of argument that it is discovered that the average intelligence of the army is 12 years-the level of the high grade feebleminded, high grade moron. We could conclude that the average intelligence of the whole population is not much, if any, above this, since the army of drafted men is prob- 
ably a fair sample of the general population. We seem to be impaled on the horns of a dilemma: either half the population is feeble-minded; or 12 year mentality does not properly come within the limits of feeble-mindedness. Of the two possibilites most people would take the latter. Should then all who test 12 be turned out of our institutions? We do not think so. Such findings would compel us to look for another factor than mental age as determining which of these persons of 12 year mentality need the care of an institution for the feeble-minded. Indeed this factor is already recognized.

Every careful student of feeble-mindedness knows that there are thousands of people of 12 year mentality who are getting on in the world and who do not need to go to an institution for the feeble-minded. What is the difference between these and those who must be cared for in an institution? There is no definite answer. It may be any one of several factors. It may be that vague something that we call temperament. It may be the accident of a favourable environment. In any borderline condition we often see the status determined by a slight influence. A person near the climax of a disease is frequently saved or lost by the slightest favourable or unfavourable circumstance. One who has inherited a constitution favourable to tuberculosis may by judicious selection of environment (or it may be accidental) entirely escape; or he may carelessly get into an environment that will surely prove fatal. Undoubtedly many morons are in a favourable environment.

This brings us to the view that we have expressed elsewhere $(20 \mathrm{~B})$ that perhaps moronity is a problem of education rather than of segregation. The probable discovery that the average intelligence of the army and the 


\section{MEASURING INBORN CAPACITY}

general population is low-even if not so low as 12 years-would certainly force us to this view and to this solution. The formula would then be: recognize the mental level of each person; understand its significance; educate accordingly.

The theory of measuring intelligence has sometimes been misunderstood; and even the possibility of such measurement has been denied. Judge Goff of New York is reported to have said in connection with a case brot before him, "You can no more measure intelligence than you can measure electricity"!

Needless to say that errors of this kind are due to a misunderstanding of the underlying principles of the measuring scales, and to a misconception of the nature of mind. The first difficulty comes from the fact that we more or less unconsciously assume that in measuring intelligence we are measuring a child's attainments. As a result of this error it is constantly objected that "children differ so much in their opportunities," "in their schooling" and "in their environments" that it is impossible to compare them. One even hears the test questions themselves objected to on the ground that "my child has never been taught that." The irrelevancy of all this appears when it is understood that in using mental tests we are testing, not attainment but inborn capacity.

If we could inspect the brain of the living subject and determine that in certain areas the neurons were not developed, the fact would be accepted as easily as the physician's diagnosis when he says, "This boy's heart is weak." Since we cannot examine the neurons we are limited to noting how they function. But from what we discover in that way we can infer the condition of the neurons just as tho we had seen them. 


\section{PSYCHOLOGY OF THE NORMAL AND SUBNORMAL}

The theory of mental tests is that they measure inborn capacity and the success of measuring scales depends upon the skill with which tests are found that depend for their solution upon inborn capacity and not upon special attainments. For example, it is well known that the ability to walk depends upon the development of a neuro-muscular co-ordination which is complete normally at about fourteen months; that no amount of training can hasten this development and that nothing but the most extremely unnatural environment can prevent it or retard it. The child of the untutored savage learns to walk as quickly as a child of the most cultured parents in the highest civilization. Playing the piano, however, is an attainment, an accomplishment, and comes only as the result of definite purpose and practice. If, therefore, we were examining a ten year old child and found that he could not walk, we should properly conclude that there was something wrong with his development, but the fact that he could not play the piano would have no significance whatever, merely indicating that here was an accomplishment to which he had not devoted himself.

There is no better evidence of the limitations of our psychology than the recognized inability to decide beforehand whether a particular test measures this inherent capacity or is influenced by training and special environment. We can indeed rule out a great many tests, such as piano playing, as obviously a matter of education; but beyond that we are largely restricted to the laborious process of trying out each test with properly selected groups of children and thus ascertaining to what extent training affects the test.

Nowhere does the genius of Binet show more clearly than in the success with which he selected tests, which 
experience has proved are of the right kind. It is true that not all of his tests are entirely free from the influence of training; but very few, if any, are so much influenced as to be useless. Perhaps the best illustration of an ideal test is Binet's "copying the square" and "copying the diamond." Experience seems to demonstrate clearly that the ability to do these tests depends upon a neuromuscular development either of the eye or of the hand or both, somewhat comparable to the co-ordination necessary for walking. A child can copy a square at the age of five, he does not copy the diamond until the age of seven. The difference is apparently due to the fact that horizontal and vertical lines are both seen and drawn by relatively simple muscular co-ordination, while oblique lines require a double co-ordination both for seeing and for drawing. That it is a matter of eye movement in following the lines more than of hand movement in drawing them, would seem to be indicated by the fact that many a child who has drawn the square with reasonable accuracy declares the moment he looks at the diamond, that he cannot draw it. Often he will not even try to draw it.

The accompanying illustrations Fig. 48, are reproductions of the attempts of children of ages five, six, seven, and eight years to copy these forms. A two-inch square is placed in front of the child and he is asked to draw one like it; then a diamond of the same size is given him. Plenty of time is allowed. Often the child is urged to make successive trials tho only the first is scored ${ }^{1}$ except when he himself sees his failure and asks for a second trial. It rarely happens that the second trial is acceptable tho sometimes a little better than the first.

1 Terman gives three trials and scores the best one. 


\section{PSYCHOLOGY OF THE NORMAL AND SUBNORMAL}

The reader is urged to study these forms carefully. Note the good square and beside it the same child's failure on the diamond; these failures ranging from almost success (one or two were credited to the child's score tho counted here as failures to make sure that the percentages were conservative) to absolute failure-a mere line. The beginning is often good; frequently the upper half of the diamond is excellent only to be spoiled by a single line, straight or curved, for the lower half. The last "diamond" in second column of six-year old children is a type frequently found. It shows lack of coordination necessary to make the hand go where the eye shows that it should go-compare your own attempt to draw a diamond seen in a mirror.

That these two tests depend upon inherent eapacity and not upon training is evidenced by two facts, first, the normal child of eight years or older copies the figures without ever having been taught; and second, a feebleminded child or a child just a little below the proper age as a rule cannot be taught to copy them.

A study of this point by Miss Elizabeth Foote of the Vineland Laboratory gives the following results:

Mental Age

Number of Cases

Per cent. trained

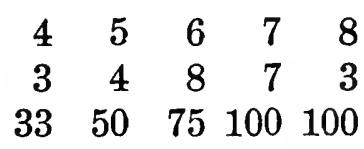

Apparently the chronologically older cases have greater difficulty in learning to draw the diamond. At mental age 5 the failures average 17 years chronologically, the successes average 11 years.

At mental age 6, the failures average twenty-nine years chronologically, the successes average eighteen years. 
$\Delta_{\Delta} \Delta \Delta \Delta \Delta \Delta \Delta \Delta_{\Delta} D \Delta_{\Delta} \beta \Delta$

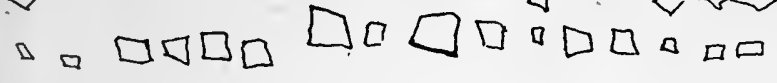

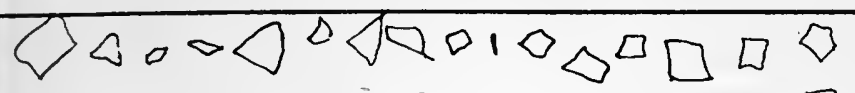

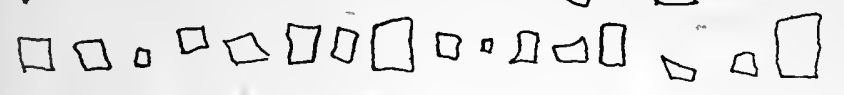

andsascos 100000 $\square \square \square \square \square \square \square \square \square \square \square ० \square \square \square$

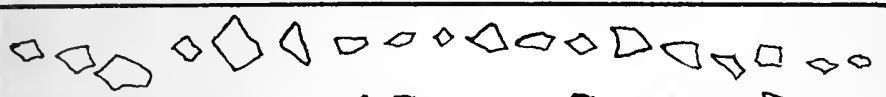

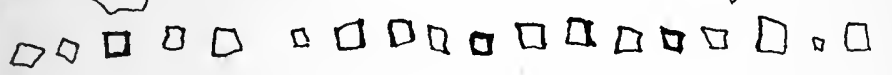

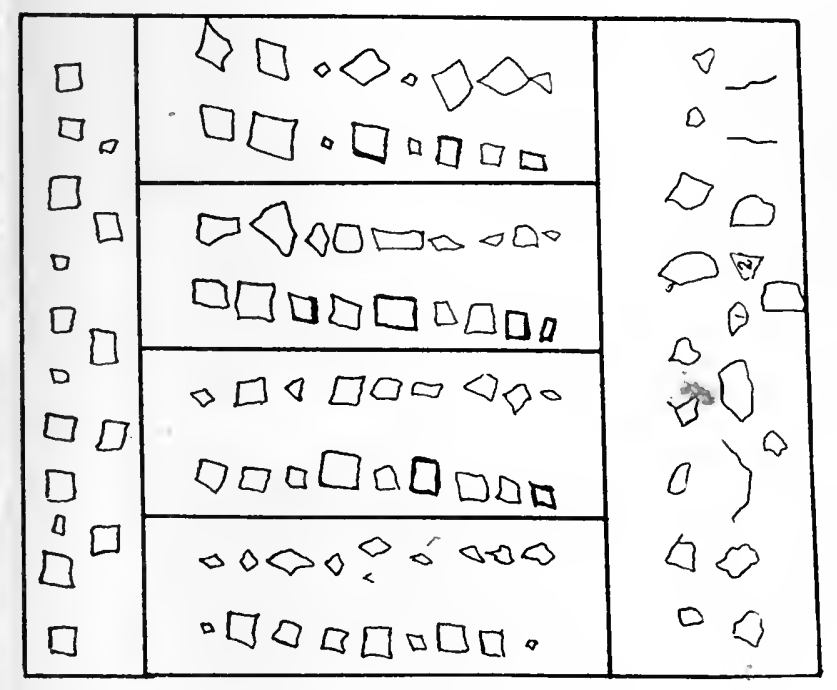

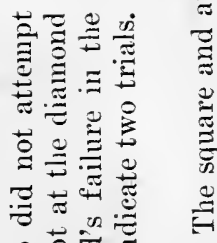

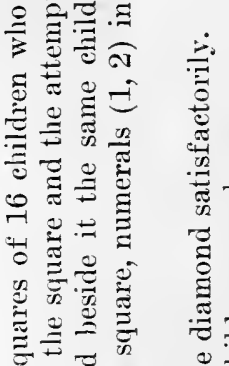

范芯导

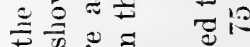

范芯芯

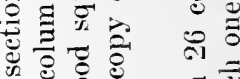

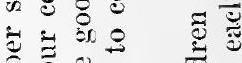

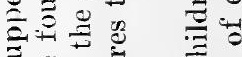

[Ð

5...ำ

$\bigodot_{-1}^{\infty}$

馬.

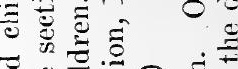

율 导导

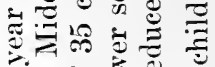

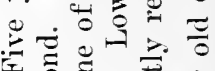

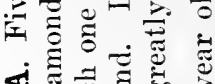

ব.

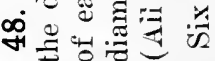

$\operatorname{sim}_{i=1}$

$\dot{\varphi}$

\&

$\sin _{101}$ 


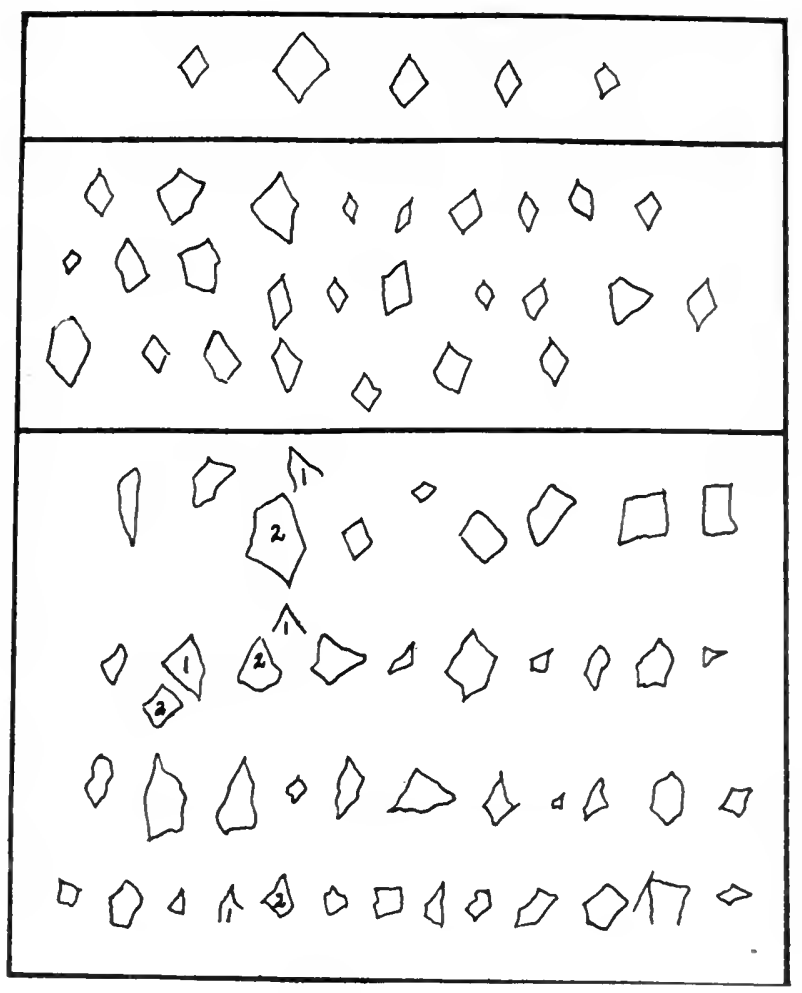

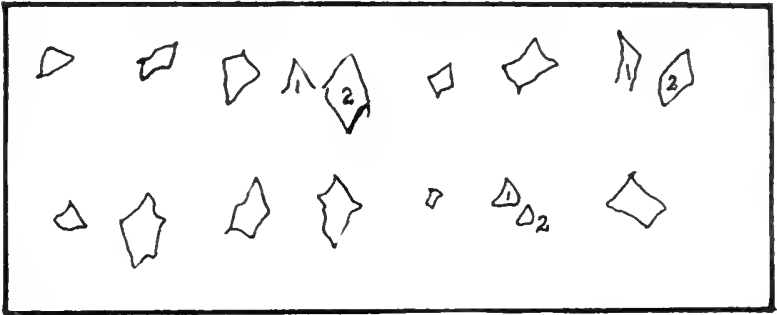

Fig. 48. C. Seven year old children. of 320 children 256 copied the diamond satisfactorily. Upper section shows 5 samples. Middle section: 26 more or less unsatisfactory. Lower section: 11 failures.

Fig. 48. D. Eight year old children. Shows the 14 failures out of 150 children. The remaining 136 copied diamond satisfactorily.

From original records in the Vineland Laboratory 
The 4-year olds (mentally) who failed were absolutely unable to draw the oblique lines.

The average time required to teach those who finally succeeded was: Mental age 6, 44 minutes; Mental age 7, 26 minutes; Mental age 8, 20 minutes.

Let us examine a few more of these test questions with the view to clearing up some of the difficulties commonly met with. Binet has introduced four questions involving the development of the ability to appreciate time. These questions have been as much objected to as any in the list, apparently because the real nature of the test is not understood. Because we sometimes teach the days of the week, days of the month, months of the year, it is assumed that they would never be learned if we did not teach them.

Binet discovered that the appreciation of the passage of time develops with age. To the young child time is nothing. Gradually he comes to appreciate time in small quantities. As his development proceeds he has need for measuring time in increasingly larger quantities. The first stage is the appreciation of the difference between forenoon and afternoon. This comes at six years of age. In other words the child of six, living in any ordinary environment, has acquired enough experience to find it useful to make the distinction between morning and afternoon. If, in the morning, he is told that he may do something in the afternoon, he is able to appreciate when that will be; or in the afternoon he can recall what happened in the forenoon of that day. Because that is useful to him, it gets into his consciousness as a part of his working experience. But he cannot yet, nor can he for two years, comprehend so large a division of time as a week. Consequently he has no use for Mondays and Tuesdays and Wednesdays. At the age 


\section{PSYCHOLOGY OF THE NORMAL AND SUBNORMAL}

of eight, however, he has developed to this stage and without ever having been taught, he has, thru the practical experiences of life, learned that Tuesday comes after Monday and then comes Wednesday, and so on.

It is unfortunate perhaps that, thru a mistaken pedagogy, we are in the habit of teaching the days of the week. We say unfortunate advisedly because it is a waste of time, has no value for the child; for, altho he can recite the days of the week, they mean nothing to him until he has developed to that stage where he appreciates this larger period of time and realizes the value of sub-dividing it into days each with its own name. When he has arrived at that development he will learn the days of the week without being taught them. In another year his appreciation of time has enlarged so that he has some use for the divisions of the year. Previous to nine years of age, years are little more than words to children, and months as divisions of the year are meaningless. Gilbert, mentality 7 , age twenty-seven, being asked how often Washington's birthday came, said he thot once in two years. This is pure verbalism; he had no conception whatever. With this appreciation of the year and the division into months the child has all the elements for the date; so that at the age of nine he is able to give the exact date with reasonable accuracy; that is, with that same leeway of two or three days which almost any of us may at times require.

Objection has been made to this test, based upon the superficial observation that we adults sometimes, when on a vacation, pay no attention to the passage of the days and lose count; therefore, why expect a child to know. Aside from the general fact that there is no arguing from the adult to the child, we have the very interesting 


\section{MISCONCEPTIONS}

fact that the part of the date most difficult for the child is not the day of the month (within two or three days, as we have already indicated) nor yet the month itself, but the year. One often gets the answer to the question: "What is the date?" "The 20th" (correct). "Yes, what month?" "I don't know." A little older child will say; "April 20th." "Yes, what year?" "I don't know." Sometimes it goes like this: "What is the date?" "The 20th." "Yes, what month?" "April." "Yes, what year"?" After some delay "1918." If it is January or February, 1918, he may say 1917. Probably no adult in his right mind ever had sufficiently long vacation to forget what year it was!

There is no doubt that the average normal child develops these capacities at about the ages stated. Therefore these questions in regard to time are valuable measures of the child's development. It is true, as indicated, that teaching may enable the child to recite days of the week and months of year some time before he has the actual appreciation of their meaning. This can usually be checked, however, by a little questioning such as, "What day comes before Wednesday, etc., or what month comes before December?"

These tests also illustrate another point that is often missed when considering tests of intelligence. Some persons have felt it desirable to analyse the tests and determine what is tested by each question; whether memory, attention, imagination, reasoning, judgment, or what-not. We have explained what the above questions test, but to show that they do not test mere memory, the following experience may be worth recording. "A child who was being examined was asked, in course, to give the date. He was totally unable to do so. The child's teacher, who 
was observing, interrupted to say, 'Why, Willie, you know the date. What was it you wrote at the head of your paper this morning?' Whereat the child gave correctly the date. When it was written down, the teacher. drew a circle around the figures indicating the year and pointing to it, said to the child, "What does that part mean?' He looked at it intently for some seconds and then said inquiringly, 'Spring'? It is evident that the child, given the proper cue, remembered what he had written at the top of his paper that morning. This to the teacher was the date, but to the child it was nothing but words and figures. He could not give the date because he had not come to that mental development that enabled him to grasp and appreciate the fact that time is divided into years, months and days."

We have emphasized the point that children acquire the ability to do many things without being taught-copy square and diamond, appreciating time-forenoon, afternoon, days of week and months of year. Another study by Miss Foote shows this very clearly. The opposing of thumb to fingers is rarely if ever taught. Yet it is seen to have a very regular development. The following table shows the results of testing 176 normal Public School children and 111 feeble-minded children. Public School children were from the first four grades, and defectives mostly between the chronological ages of eight to forty-seven.

It will be seen that all Public School children can do this at age seven and all defectives at age eight. It is also seen that sight helps only a little-the positions out of sight-behind back-being done by almost as many is those in front. Both hands at once is also a little more difficult than one at a time. 


\section{THUMB-FINGER OPPOSITION}

Thumb-Finger Opposition. Percent Perfect.

Public School.

$\begin{array}{ccccccc}\text { Age } & \text { R. Front } & \text { R. Back } & \text { L. Front } & \text { L. Back } & \text { Both Front } & \text { Both Back } \\ 5 & 91 & 80 & 94 & 74 & 74 & \mathbf{6 0} \\ 6 & 98 & 93 & 98 & 93 & 83 & 90 \\ 7 & 100 & 100 & 100 & 100 & 100 & 100 \\ 8 & 100 & 100 & 100 & 100 & 100 & 100 \\ 9 & 100 & 100 & 100 & 100 & 94 & 94 \\ 10 & 100 & 100 & 100 & 100 & 100 & 100\end{array}$

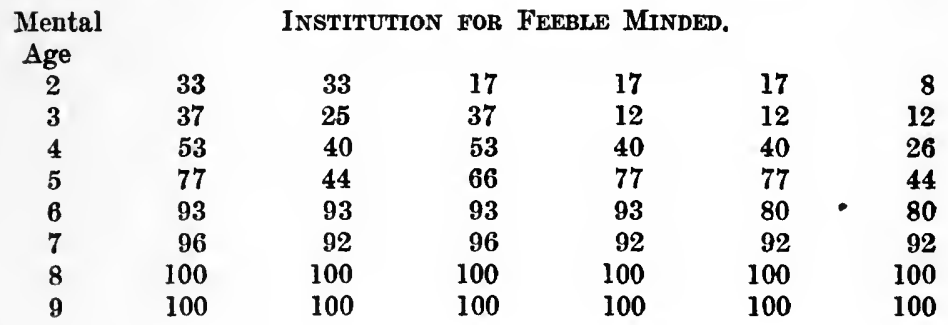

The reader will do well to keep in mind that measuring scales of intelligence test intelligence; not attention, or memory, or reasoning, or any other one thing.

It has already been suggested that intelligence is adaptation to environment. If we accept this, we must conclude that a test of intelligence is a test of a person's ability to adapt himself to a situation; and the grade of intelligence, the mental level, or mental age, is determined when we ascertain the highest degree of complexity of environment to which he can adapt himself, in terms of the age of the average person who can just adapt himself to the same environment. To revert to our questions of time, when we ask a child, "What is the date?" we are attempting to ascertain if he can adapt himself to his environment as well as nine year old children usually can, when they need to use the date.

We have said that tests of intelligence do not test memory, reasoning, etc. To avoid misunderstanding let 
us explain more fully. Tests of intelligence of the higher levels must necessarily test ability to make adaptations that involve the use of the mental processes memory, imagination, reasoning, etc. But this is very different from testing these processes themselves. Let us take a somewhat imperfect analogy. Suppose we wish to measure a man's efficiency as a citizen-good citizenship. We ascertain how much money he has; what knowledge of civic duties, of public affairs, of science, of esthetics; what his ideas of honesty, truthfulness and friendship are; and as many more "elements" as we wish. Have we measured his citizenship? Obviously not. The real question is, how does he use all these? He may have millions of money, but if he never uses any of it for civic purposes he is a poor citizen. He may have the most correct ideas of honesty, but if he does not practise it, he is not a good citizen. He may have very little money or education, and yet be high in the scale of citizenship. Not what a man has but how he uses it!

The weakness of the foregoing analogy lies in the fact that it seems to grant too much. Even if memory, reasoning, etc., were entities like money, education and the rest, the measurement of them would not measure a man's Gitelligence since the real question would be how does he use them. James sums it all up in a sentence: "The total mental efficiency of a man is the resultant of the working together of all his faculties." Therefore in measuring intelligence we must have the mind at work. This every good test does.

We have emphasized the fact that mental tests test the degree of development of the natural endowments in distinction from the acquisitions or attainments. There are, however, tests of the latter. These are called 


\section{DIAGNOSIS}

pedagogical tests, or special ability tests. The reader who has followed the psychology of Part I, will appreciate that both of these involve mentality. A child may not be up to standard sixth grade work in school for one of two reasons; either because he has not been to school, or because he has not the inherent capacity. It is often possible to evaluate the two factors. That is, it is possible to ascertain whether the child has been in school six years or not. If he has attended school six years under normal, ordinary conditions and is not up to sixth grade, it is fair to assume that his failure is due to lack of mentality unless some other explanation is known. It consequently comes about that pedagogical tests and even school experience are often a valuable indication of the mental level of the child.

In conclusion we may say that the measurement of the mental level is an assured fact. Moreover for the average child almost any one of the current scales is entirely satisfactory and gives the trained person all the information he needs. It is the exceptional case, the border-line case, or the diseased child, that constitutes the problem for the psycho-clinician. In such cases one has all the difficulties that confront the physician in diagnosing an obscure disease with complications. The greatest care must be exercised; all possible data must be secured and a diagnosis given with caution. But the fact that there are exceptional and difficult cases, and even that mistakes are made, should not deter us from extending the work of ascertaining the mental level of all children - and ultimately of all adults-for the purpose of fitting every man intelligently to his work. 


\section{CHAPTER III}

\section{INTELLIGENCE AND WILL}

In the application of our psychology to the care, train. ing and management of persons of undeveloped mind, many problems are simplified by a clear understanding of the relations of will to intelligence. Much unnecessary anxiety exists, in the minds of those who have the training of such persons, from the common belief that will is a power possessed by the individual sometimes feebly and sometimes to a great degree-a concept, it must be admitted, not wholly dissipated by current psychology.

Fortunately the old religious dogma that the child's will must be broken has almost passed away. The idea still prevails, however, that there is a will that must be carefully trained and directed and that it is so little understood as to be largely a matter of chance whether such training and direction will be successful. When it is clearly recognized that will is only an abstraction, expressive of the fact that one is capable of volitional or selective action, the difficulty largely disappears. We have seen that volitional action results when a natural impulse is met by an opposing impulse or by a tendency to an acquired action which conflicts with the original impulse.

In the words of Prof. James, "Voluntary action then is at all times a resultant of the compounding of our impulsions with our inhibitions." James has also shown that will is dependent upon ideas, attention and habit of 
action, all of which he sums up in a sentence previously quoted, "To think, in short, is the secret of will just as it is the secret of memory." From all this the conclusion is easy, that the way to control and direct the will is (1) to furnish plenty of suitable ideas, (2) to connect those ideas by proper association processes so that they will arouse consciousness in the state of active attention; and (3) to develop right habits of action. It might seem to be unnecessary to say more; but there is another consid. eration that will be helpful in this connection.

Much of what is properly considered will is psychologically only impulse. Whether there are wide differences in the strength of impulses in different individuals, or whether the apparent difference is only due to a difference in the control exercised, it is difficult to say. There seems, however, to be no good reason to deny that there may be very wide individual differences. Be that as it may, the solution of the problem, as Prof. James's dictum clearly implies, is to build up inhibitions, that is, counteracting ideas. The idiot is entirely a creature of impulse, since he has few ideas and what few he has cannot be connected with the impulses by any natural association, because he is deficient in association neurons.

In the case of the imbecile, the difficulty is only slightly decreased. He has a few more ideas, it is true, but it is difficult to connect them with the impulses in such a way that the latter are controlled. To say it is difficult, is not to say that it is impossible. It is not impossible; but it can only be done with the greatest effort, most constant attention and constant direction. The normal person sees the consequences of his impulsive act; and those consequences, being remembered, on a later occasion act as inhibitors. With the imbecile the consequences must be 
definitely pointed out and the connection firmly made by constant repetition.

With the moron the problem is more hopeful but still requires long and painstaking effort and great care in the building of proper associations. Even with the dull normals there are still difficulties, due probably more to the early formation of unfortunate habits than to any lack of ideas or failure to connect them with the impulse.

Since the ideas, attention and action upon which will depends are the elements which enter into intelligence, it follows that if we know the intelligence level of an individual we know by far the largest factor of his will. The other factor is his habit of action. (For the effect of emotion see the next chapter.) Knowing these two factors, the intelligent person can estimate to a nicety how much volitional action a particular individual will exercise in any particular situation.

Moreover, it is significant that such foresight is constantly used by intelligent people in managing their relations to other people. Take a single example: $\mathrm{X}$ owes $\$ 100$ to each of three men A, B, and C. The debts to A and $B$ are of long standing and ought to be paid. $X$ comes into possession of $\$ 100$ and has to decide which one of the three he will pay. He reasons as follows: "I'll not bother with A because he does not know how to proceed to collect the money, consequently will not trouble me. B is a lawyer and knows how to collect, but he is not in the habit of bringing suit for bad debts, consequently he will not disturb me. $\mathrm{C}$ is a hard headed business man. He knows how to collect his debts and he is in the habit of doing it. If I don't pay him he will make me trouble. Therefore I shall pay C." From the standpoint of [266] 
psychology, A and B are weak willed for different reasons -A from a lack of ideas; B from lack of the habit of action. In this particular direction, $\mathrm{C}$ is strong willed in this particular matter because he has the ideas and the habit of action.

The old doctrine of will-breaking can be defended only on the following assumptions: First, that will means impulse; second, that it is impulse toward a line of action that is indubitably bad; and third, that the "breaking" consists in building up a set of experiences which result in inhibitions that will control the impulse. It must be admitted that there are apparently many instances where it is exceedingly difficult and requires great intelligence to accomplish this result.

The Moral Imbecile. A consideration of the moral imbecile will further illustrate some of the points discussed in the previous paragraphs.

In the older literature on feeble-mindedness can be found discussions of a type of defective described as having normal intellectual faculties but lacking moral faculties. A person of this type, tho he evidently had normal intelligence, would lie, steal, commit sex offences or other immoralities in spite of punishment and all efforts to correct the tendency.

Perhaps we should first ask the question, are there such beings? To which the answer must be given, it is somewhat doubtful. It is now accepted by those who have studied feeble-mindedness, that a large proportion of the so-called moral imbeciles are not of normal intelligence. The explanation of the old view, that such persons are normal intellectually, is found in the fact that they are of relatively high intelligence, and in the earlier days when less accurate methods of measuring intelli- 
gence were known, and when the typical mental defective was the idiot or the low grade imbecile, these high grade defectives easily passed for normal, just as today the morons pass for normal with the layman.

On the other hand, it apparently must be admitted that there are persons who do not at all come within the present definition of feeble-mindedness, and yet who seem to lack what we popularly call moral sense. It goes without saying that the old explanation of "normal intellectual faculties" and "lacking in moral faculties" is no longer permissible, since neither does psychology allow that there are intellectual faculties nor does modern ethics allow the concept of moral faculties. Morals are merely volitional actions in accordance with the customs of the social group; hence this comes under the problem of the will which, as we have already seen, is mainly a question of intelligence. Logically then, a person who has normal intelligence should be able to so control his action as to be "moral" rather than "immoral." It must be admitted that little is understood of this type of immoral person and in this field little is to be gained from speculation.

Future studies of this problem will probably have to deal with the habit of action and must ascertain whether these are cases where an unusually strong habit has been developed before attempts were made to supply ideas and furnish inhibitions. Also investigations must be undertaken to determine what efforts have been made to develop inhibitions. The question inevitably arises when one meets a "moral imbecile," "What efforts have been made to correct these tendencies? Was the child ever punished? Did he ever suffer the consequences of his action?" The answers that one gets are usually either 
vague or of uncertain application. One father says, "Yes, I have whipped him until I was ashamed to whip him any more." In which case one has the natural doubt as to whether whipping is the kind of inhibition that should have been applied.

Healy has introduced the expressions pathological liars, and pathological thieves. Perhaps we should consider these cases also pathological. Perhaps there is a lesion in the brain which has destroyed the specific association neurons (if there are such) which should associate action with its consequences and thus bring about the inhibition. It is a possibility that our concepts of arrest of development might be made to cover the case by assuming such a definite localization of the arrest as to account for the condition found. That is to say, instead of there being a general arrest of development of the neurons of the association areas, there may have been a narrowly limited, localized arrest of certain fibres which connect the centres that give rise to the impulse to lie or steal with those centres which give the consciousness of consequences. This, however, is a difficult concept in view of what we know of the growth of the cortex, and until we have more definite clinical evidence it can give little satisfaction. 


\section{CHAPTER IV}

\section{INTELLIGENCE AND EMOTION}

IN the previous chapter we have discussed some of the implications of the relation of intelligence to will. We now come to the consideration of a problem that is still more in need of elucidation. We perhaps cannot better bring the problem before the reader than by quoting the oft heard remark, "It is not enough to know a child's intelligence, his mental level, but we ought also to know his emotional life. Tests of intelligence are not enough, we must also have tests of the emotions." This view has far more to justify it than the views about the will already discussed.

While to the lay mind the part that the emotions play in human life may not seem to be any greater than that played by the will, yet when we come to the physical basis of the problem we find an absolute difference. There is nothing in the nervous system that can by any possibility be considered the organ of will, but there is a very definite, elaborate and complicated nervous system that has been definitely recognized by Mosso as the "seat of the emotions"; namely, the sympathetic system. Moreover, definite connections have been established, as we have already shown, between this sympathetic system and the glands of internal secretion, by which they are known to react upon each other. Since it is easy to conceive of differences in structure and function of the sympathetic system and the glands, and since we have the clinical evidence in at least one case,- that of the effect of the 


\section{INTELLIGENCE CONTROLS EMOTION}

absence, or the hypertrophy of the thyroid gland producing the symptoms recognized as cretinism,-it is not only logical, but we are practically forced to accept the view that there must be enormous individual differences in the emotional life of individuals and that such differences, being fundamental and inherent, are not easily regulated but must be reckoned with. From this it seems logical to conclude that until we can understand conditions in this realm and analyze and measure the emotions, it is unsafe to conclude that we know all about the individual.

However, the situation is not nearly so bad as it at first appears. It is a common experience in all science, when there are two factors, one of which is unknown and uncontrollable, to more or less successfully control the situation by manipulating the other factor. A chemist has a solution and finds by simple tests that it contains an acid. He does not know what acid or how much of it is present. He can neither filter it out nor allow for it, but he can neutralize it and render it harmless by adding an alkali. It is one of the fundamental facts of psychology that there are two phases of mind, the intellectual and the emotional, each so important and so strong that we commonly speak of an emotional life and an intellectual life.

But a further fact is not so commonly recognized; namely, it is a scientific fact and a common experience that the intellectual life regularly controls, to some degree, the emotional life, and probably is capable of complete control of action no matter what the condition of the emotion. From this the conclusion is inevitable that if there is little intelligence there will be little control, whether the emotional life is strong or weak. If the chemist's solution is decidedly acid and he has only a 
single drop of weak alkali, we can predict with certainty that he will not be able to neutralize or control the acid.

We have drawn some hypothetical pictures for the sake of illustrating how the neuron action of the cerebro-spinal nervous system influences, and is influenced by, the sympathetic system. We shall not go further into that subject at the present time, but shall confine ourselves to reminding the reader of some of the instances which show (1) that the intelligence controls the emotions and (2) that the emotions are controlled in proportion to the degree of intelligence. It follows that if there is little intelligence the emotions will be uncontrolled and whether they be strong or weak will result in actions that are unregulated, uncontrolled and, as experience proves, usually undesirable. Therefore, when we measure the intelligence of an individual and learn that he has so much less than normal as to come within the group that we call feeble-minded, we have ascertained by far the most important fact about him. While not denying that it would be highly desirable to know more about his emotional life, to be able to measure it and evaluate it and determine its influence upon the intellectual life, we may yet satisfy ourselves that a knowledge of the intellectual condition gives us, within limits, enough information for our guidance in the care, control and regulation of such defective individuals.

We have already cited, page 146, McDougall's view of the way in which the primary emotions are normally modified. We have also called attention to the wonderful power of ideas in increasing emotions. Let us consider still another case. Three or four people are conversing; one of them utters a sentence which produces practically no effect upon $A$, arouses a strong sense of 
humour in B, and an intense emotion of anger in C. The explanation is obvious. The resultant emotion or lack of emotion depends upon the ideas with which each one has received the remark. A has no experience which can combine with the remark and make it especially significant. In B it calls up a whole train of ideas and experiences so connected, so related to him and his welfare, that it arouses an intense degree of pleasure. C, on the other hand, has had the opposite experience. He may see in the remark a significance that touches him or his family in a way that means decided annoyance or discomfort. $\mathrm{He}$ is accordingly angry. Without their respective experiences $\mathrm{B}$ and $\mathrm{C}$ might have been as passive as was $A$. It is unnecessary to multiply instances, the experience is so familiar that every one must recognize it.

Let us take a little different example and see how the situation and experience modifies the emotion. A man is alone in the forest and meets a wild and ferocious animal. He may easily be "paralysed with fear" but if he has a gun in his hand he will probably exhibit scarcely even a trace of fear; while if he is hunting that particular animal, the sight will produce intense joy. Consider for a moment the sex emotion, supposed to be the most uncontrollable of all human instincts; yet it is notorious that the intelligent man controls even this. While there are intelligent men who claim that they have such a strong instinct in this direction that they cannot control it, and this is their excuse for an immoral life, undoubtedly, if we could measure and ascertain the facts, we would find that there are just as many men with just as strong sex impulses who do maintain complete control. Because they are intelligent, they appreciate their relations to 
their fellow men and are able to bring to bear upon every situation sufficient inhibiting ideas to enable them to refrain from acting immorally.

The writer has seen idiots who had suffered pain from having teeth extracted and were, upon another occasion, terrified at the visit of the dentist. The imbecile seats himself in the chair with fear and trembling but with no terror. The moron has sufficient intelligence to know that his teeth are not hurting him and that probably none will be extracted; and so he sits down, opens his mouth, submits to the examination with calm composure. The normal man, with his superior intelligence, seeks the dentist, asks for an operation that he knows will be painful; yet his mental state is one of real satisfaction, not to say joy, because he knows that it will be for his benefit finally.

Twelve years' association with the feeble-minded, and study of their condition has convinced the writer that the fundamental emotional conditions are not so variable as is commonly supposed. The feeble-minded want to be comfortable and happy and they are not hard to please. When treated in a manner to produce happiness, they seldom manifest violent emotions. In all these years the writer has never seen such paroxysms of anger as he has seen among normal boys. Even the sex instinct is much less in evidence among mental deiectives than among a similar group of normal persons. The social relations between the sexes in most institutions for the feeble-minded are much the same as in a co-educational boarding school. The boys' cottages are separated from the girls' cottages; many of the boys at their work or on errands come in contact with the girls; in some of the shops they work together; they are together in school; 


\section{THE MORON NOT VICIOUS}

they all meet in the same hall two or three times a week for entertainments, Sunday assembly, etc. Yet in the institution best known to the writer, there have been in twelve years not more than two or three instances that indicate any special sex activity, and those were relatively unimportant. If one compares this with conditions in public schools or co-educational schools, the conclusion seems to be inevitable that the feeble-minded do not have abnormal sex impulses.

The reader must appreciate the difference between the fundamental, natural strength of the instinct, if we can so speak, and acquired conditions. We have dwelt upon the fact of the modifiability of the instincts, both in the direction of strengthening and of weakening them. The instincts and emotions of the feeble-minded may be strengthened, as is evidenced from the experiences of the feeble-minded who are not cared for in institutions and made happy. It is commonly believed, by those whose only experience is with morons outside of institutions, that they are a dangerous, vicious, bad group of people, full of the strongest instincts and emotions. But we can only conclude that this is the result of their having had their emotions stirred and developed by the necessity of reacting against unfair treatment which tended to make them unhappy and consequently resistant to all efforts at control. The feeble-minded person is not naturally wicked or bad, but when misunderstood and mistreated, he does have enough of the primitive human instincts to react; and constant reaction to bad treatment does develop his emotions to a strength not usually found in those of higher intelligence who have been more wisely treated.

Thus the facts of every day life, when carefully inter- 


\section{THE MORON NOT VICIOUS}

preted, seem to point to the view presented, .hat while action is modified and reinforced by the energy of the sympathetic system, which appears in consciousness as emotion, yet it is intelligence that modifies and controls the action of the sympathetic system or, as we say for brevity's sake, the emotions. 


\section{CHAPTER V}

\section{EXPERIENCE}

THE reader who has followed our discussion and explanation of mental processes will have noted the fundamental part played by experience. The normal human organism includes an elaborate nervous system with either actual or potential neuron patterns capable of responding to all the important stimuli and directing all the necessary actions of life. But even those inborn neuron patterns cannot function without being set into activity by an ap-. propriate stimulus. Indeed, there seems good reason to believe, that if the stimulus is never presented, these specific neuron patterns either degenerate or become so subordinate to other patterns that are exercised, that for all practical purposes it is as tho such patterns did not exist.

But we have seen that while the inborn neuron patterns form the starting point, the basis for reflexes and instinctive actions, and probably cover all those activities that are vitally necessary for the perpetuation of the race and for the safety of the individual under simple conditions; yet, the ability to protect one's self in a more complicated society, that is to say, the capacity for what we have called the higher mental processes, elaborate thinking, reason, judgment, will and all that constitutes the higher levels of intelligence, depends upon the grouping together of these relatively few primary instinctive patterns to form larger and ever more elaborate and complex neuron patterns. 


\section{PSYCHOLOGY OF THE NORMAL AND SUBNORMAL}

We have also seen how this whole extension and elaboration is brot about by experience. Perhaps nowhere is one brot so face to face with the importance of experience as in the consideration of imagination. We have found that imagination, while popularly supposed to be unlimited in its functioning, is, on the contrary definitely limited by the experience of the individual. Ideas are exceedingly useful in the elaboration of thot and reasoning and judgment; but "ideas arouse experi.. ences." Without the experience back of the idea there can be no neuron pattern that can function as a part of the elaborate pattern that we have assumed underlies thot.

This fact seems to be generally recognized by all intelligent people except some teachers and trainers of children. There is, for instance, a sharp distinction between book knowledge and practical knowledge; between knowing about a thing and knowing the thing itself. We say of a man, he has had large experience, or he has had no experience, and our estimate of his efficiency is determined by this fact. The average college graduate is notoriously helpless and useless when he knows things only from books and has had no practical experience. The intelligent man who takes up any new line of work prepares himself by seeing what others are doing, by getting experience. Hence, the vast amount of travel and visiting. The school superintendent visits other school systems. The teacher visits other classes; the manufacturer visits other plants to get new experiences so that he will have ideas. Societies are formed by the leaders in every industry, occupation, and profession, for the exchange of experiences. All of which comes back to the fundamental fact that no man can imagine new lines of activity without first getting new experiences. 


\section{EXPERIENCE}

And yet the teacher, and often the parent, thinks that he educates and trains the child by telling about things.

The mother who thru fear of accident keeps the child close at home, or the father who is too busy to take his boy with him and show him things, is dooming that child to incapacity and inefficiency vastly more than he would be by keeping him out of school and not allowing him to learn to read. Now the positive side of all this is, that immature mind gets its development and training thru experience. Therefore, every provision should be made consciously and definitely for giving the child all possible experiences that will help to build up useful neuron patterns. The child should see everything, hear everything, taste everything, smell everything, handle everything in order that he may have a complete equipment of sensations, perceptions and experiences that he can use and build up into his more complex creative imaginations. All these experiences should have a certain orderly or logical arrangement, so that they can be found when wanted. This orderly arrangement of experiences is science, enabling one to meet the needs of life. The lack of this makes the difference between a department store and a junk shop. A junk shop is infinitely better than an empty barn, but efficiency would be greatly increased if the articles in the junk shop were classified and arranged according to some logical principle. So it is with experiences. Without experience, one's mind is an empty barn. The untrained man of affairs who has picked up his experiences by himself is apt to have a mind that is more or less of a junk shop; valuable, and often relatively efficient, he would have been vastly more efficient if he could have been scientifically trained. It is the business of formal education to supply the neces- 
sary experiences and to furnish them in logical arrange. ment.

In the previous chapter we have used the term vicarious experiences, meaning those cases where we use the experience of another. This must be explained, else it will lead to just the practice that we are condemning. It is the unwarranted confidence in the value of the experience of others that has led to our careless practices in education. We can use the experiences of others, but only to a relatively limited degree. The limitations must be understood.

The most important condition for making use of another's experience is that one shall have had some experiences that are somewhat related to those by which he wishes to profit. He must have had the details of the other's experience tho he has not experienced all the elements together. In other words, we are apparently dealing with the same problem of imagination. I can build up an imaginary picture of my friend's experience if I have some time myself experienced the details. I may never have seen a certain institution for the feebleminded, consequently I cannot reproduce any image of it. But if a friend describes it to me as located on sloping ground, at the foot of rather high wooded hills, composed of a large number of buildings of red brick, the separate buildings connected by wooden corridors and arranged around a square court, and so on, I can imagine the institution because I have actually experienced all the details mentioned. My image will be accurate in proportion as my friend has gone into details accurately, and as I have carefully attended to all he said and reproduced each image with reasonable completeness. Thus. I have a vicarious experience of that institution.

[280] 


\section{EXPERIENCE}

All this information may be conveyed to me with considerable degree of accuracy, and very quickly by the presentation of a photograph; and in proportion as I study and understand the photograph, I shall have a fairly good experience of the institution. It will be seen at once that we have an immense amount of this kind of experience, but it will be admitted almost as quickly that such experience is never quite equal to firsthand experience. Occasionally one has an experience of something that he has built up in imagination, and he is able to say, "This is exactly as I had pictured it; seems to me I have been here before." But it is comparatively rare that one can have this feeling. Vastly more frequent is the discovery that the actual thing is very different from one's imaginary picture. As already impled, this discrepancy arises as a result, first, of lack of actual experience of details; and second, of failure to attend closely to the description or to study the picture; or third, of actual inability, due perhaps to lack of neurons, to bring together all these different parts into one co-ordinate whole.

From these considerations it is possible to see clearly where we fail in our attempt to use vicarious experience with immature minds. First, we take no account of the fact that the child has not had the elementary experiences that go to make up the picture. And we very often make no effort to ascertain that fact. Secondly, we are apt to be extremely careless in enumerating the details. And thirdly, it is almost impossible to assure ourselves that the child has attended to these details and has associated them together into a complete whole.

Still more reprehensible is the practice of relying upon a verbal description and being quite satisfied if the child 
is able to repeat our language. Worse still, perhaps, is the use of symbols other than words; such as maps, diagrams, drawings, etc., which in no way represent the original experience, but only symbolize it. Such is the method of teaching geography by means of map questions, now fortunately largely eliminated in our best schools. There are many people to whom Paris, London and Berlin mean simply little black dots on a map; and the rivers Seine, Thames and Spree are black lines on the same map. These are cases where the attempt to give children the vicarious experience has proved a failure. But the most unfortunate part of it all is, not that the child has failed to get anything useful, but that he is himself misled into thinking that he has gained knowledge.

The writer once saw a class of fifty Normal School girls tested as to their knowledge of the St. Lawrence River. More than half of them declared that the water in the St. Lawrence flows southwest. When reminded that the river connects the ocean with the Great Lakes, more than a quarter of them still persisted that the river flows from the ocean into the Lakes. It is easy to see how this confusion came about; the river was to them merely the line on the map, and since this line runs from near the top of the map toward the bottom, and water does not run up hill, it must be that the St. Lawrence River flows down from the Atlantic Ocean to the Great Lakes. But in spite of all the bad teaching, we may admit that it is only bad teaching and that under good conditions the child may be enabled to profit by vicarious experience to a large extent.

We have been speaking of normal children. When we turn our attention to the feeble-minded, the problem is 
quite different. The reader has now a clear picture of the feeble-minded child with his undeveloped association neurons; from which it follows that the defective is incapable of bringing together and associating the different elements of his experience, which would go to make up the new picture. But more than that, he is usually deficient in the elementary experiences. Tho he has lived in the same environment as his normal brother, because of his undeveloped neurons the environment has not made the same impression upon him. The sensations that came through his eyes and ears have not meant for him the definite experiences that they have meant for the normal boy; therefore, the vicarious experience is almost nil for the feeble-minded boy. Moreover, if we would be sure that he gets the elemental experiences, we must be very careful to see that the stimuli make sufficient impression upon him to arouse what neuron patterns he possesses. Hence his training must be a radically different process from that of the normal child. It must be concrete and never abstract. This necessitates a definition of these terms.

It has been customary to define concrete as a term applicable to anything that exists; abstract, to the nonexistent. Pedagogically the definitions are incomplete. They should add the idea of relationship to the individual. What is concrete to one person who has had the necessary experience is abstract to another who has had no such experience. If I have never looked thru a microscope, brain cells are as much abstractions to me as atoms, hormones or goodness. The following experience will illustrate this. John, aged thirty-five, mentally 7, was being examined by the writer as to his proficiency in numbers. 


\section{PSYCHOLOGY OF THE NORMAL AND SUBNORMAL}

"John, how many are 7 and 6?" No answer.

"How many are 3 and 2?" "Don't know."

John evidently cannot handle abstract numbers.

"How many are 3 loads of coal and 2 loads of coal?" "Five."

"Good! Suppose you give your horse 6 ears of corn-"

"We don't give him six ears of corn."

"Well, just suppose you did."

"But we don't."

"Well, let's play you do."

"No, I can't, because we don't give him six ears."

(Note that John cannot imagine what is outside his experience. Clearly we must come to his terms.)

"Well how many do you give him?"

"Oh, ten or twelve."

"Very well, suppose you start to give him fourteen ears of corn and the hostler says give him only twelve. How many ears will you carry back to the bin?"

"Two" (promptly).

"Good." Here followed some other combinations, then this: "John, suppose you took fourteen loaves of bread from the bakery to the kitchen, and when you got to the kitchen the cook wanted only twelve, how many loaves of bread would you carry back to the bakery?"

John (very slowly and doubtfully). "Three?"

John had never handled loaves of bread. The problem was abstract.

John hauls coal-two loads in the forenoon and two in the afternoon.

"John, suppose you have four loads of coal in one bin and three in another, how long will it take you to haul it?"' 
"A day and a half and then a load" (promptly).

John had had the necessary experience.

The following account of Lewis's attempt to make a concrete experience out of an abstract test is a further illustration. Lewis is twenty-five years old, mentality 8. The Porteus tests are a series of mazes, the first very easy, the later ones difficult. The test is to trace with a pencil the most direct way out, and without touching the sides. An $\mathrm{S}$ shows where to start.

Lewis's experience is clearly in evidence. He knows Scranton, Pa., from experience, not from geography, which he has never studied.

Normal children often give a "setting" to their tasks, but it is not often so clear that it is a real help to them in making the task concrete.

\section{Lewis and the Porteus Tests}

The examiner had explained what was desired, but the boy could not seem to understand in the least. All of a sudden he looked up quickly, saying:

Lewis. Oh, I see, it's a train! $\mathrm{S}$ stands for station, don't it?

E. That's right. It's a train starting from the station and you're running the train. Now keep your train on the main line and don't sidetrack.

Lewis. All right. I'll take Ethel home to Washington. (Finds opening at the finish.) Here it is. It's a terminal. Write Washington there, please. Thanks. Here we go-Toooot-Pssseee-Chu, chr, chu, chu (etc. to the end of the line without a mistake). Here we are. Ethel got home without getting hurt. Washington, Washington, all change cars.

With each maze he did the same thing, taking a differ[285] 
ent person each time. When he made a mistake he would say, "Too bad, too bad. Got her hurt that time, must get her out this time or she'll have to go to the hospital and get killed."

After that he would start again and finish without a mistake. On the last test he was to take Miss W. to Scranton, Pa.

Lewis (finding terminal). Write Scranton here, please.

(Examiner wrote Scranton.)

Lewis. Write Pa., please, Scranton's in Pennsylvania. Don't you know it? You'll have to write Pa. Write it, please.

(The examiner wrote it.)

Lewis. All right. Here we go Toot-toot, PssseeeChu, chu, chu.

But he failed this one and was very much disturbed because he had "landed Miss W. in a hospital and she'll get killed and I can't get her home."

He kept worrying and talking about Miss W. and the examiner had difficulty in getting him to pay attention to the next test which was the Knox Cube. After he got the idea of it he said: "Oh, I know. This is the electric train. I can get Miss W. home on this, can't I?"

After he had been told that he could, he paid close attention and proceeded to "get Miss W. out by the electric trains."

From all this it seems that anything is abstract that is away from experience, and that the only way to deal with abstractions, at least in the beginning, is to know the concrete experience.

It is highly probable that we begin the teaching of ab[286] 


\section{ABSTRACTIONS TOO EARLY}

stractions far too early with the normal child. The defective child, who is never able to handle abstractions to any great extent, must be trained wholly by concrete material. But of that we shall speak in the next chapter. 


\section{CHAPTER VI}

\section{PEDAGOGICAL APPLICATIONS}

THE facts of the growth, development, structure and function of the nervous system, some of which have been set forth in the preceding pages, are sufficient to warrant some conclusion in regard to the training of immature minds; and many more tentative hypotheses that can either be confirmed or contradicted by appeal to experience.

Let us first consider the normal child-the child whose mind, tho undeveloped, is growing. The first principle in the development of the child is to provide for the normal functioning of his inherited neuron patterns in a way that will be most useful to him and to the social group in which he lives. This means above all else that he must have the right kind of experience, since it is experience and experience alone that sets these patterns into activity. All the natural instincts that still remain useful in modern society should be developed. Those that have ceased to be useful, and on the contrary are injurious, should be allowed to lapse thru disuse.

It is always desirable to stress the positive side by substituting a desirable action rather than the negative side by trying to destroy the bad action- "do this" rather than "don't do that." Nevertheless those instincts that begin to function in spite of efforts to the contrary must be discouraged by making the consequences so undesirable that a new neuron path will be established. 


\section{LYING AND STEALING}

For example, deceiving and hoarding are instinctive actions. They are found in animals and in primitive human beings. Where each one lives for himself, they are among the highest virtues. In the more highly developed human society they are called lying and stealing and as such are undesirable and must be shunted off.

Both animals and primitive man survive largely thru their success in deceiving their enemies and in accumulating what they need for their own subsistence. It is only when men come to live together in groups, the family and the tribe and the nation, and become mutually dependent, thru each one specializing and doing some part of the work for the common group, that truth in dealing with one's fellows becomes necessary, and the institution of property becomes developed. Under these conditions it is reprehensible to take for one's own use that which belongs to another or to deceive another of your own group.

There has been a gradual development along this line. First it was thoroly ethical to deceive or steal from any one outside the family, then outside the tribe, then the nation. The present World War is developing a world unit. Witness President Wilson's conditions of Peace -all treaties and diplomatic procedure shall be open and above board-world honesty. That even the most civilized man has not yet fully acquired these habits, to the exclusion of the old primitive instinct, is at once evident in view of certain indisputable facts. In spite of all our ethics and our ideals we still feel, and constantly hear expressed, that it is a little bit worse to lie to, and steal from, our relatives than from those outside of the family; a little worse to indulge in these practices in our own nation than with a foreign nation. Only recently has 
the ideal of honesty in diplomacy in the treatment of other nations been declared as the ideal. Moreover, once war is declared, deceiving the enemy and stealing from him become the highest virtues.

These tendencies probably come under the instinct that McDougall describes as the self-assertive. It is when the individual begins to develop a sense of self and to feel the necessity of asserting himself in his group, that he begins to work for his own aggrandizement by deceiving others as to his intentions, desires and actions, and to accumulate for himself everything that he conceives he wants. Experience teaches that this phase of the development of the child comes, on the average, at about the age of nine or ten. Naturally it appears much earlier in many individual cases and indicates its presence in certain small ways in practically all children at an earlier age than nine. Fortunately at that age there are usually enough well developed neurons, and the association centres are sufficiently developed to make it entirely possible to overcome these tendencies. This is done, as we have already explained, by building up other paths and developing habits of action that together constitute honesty and truthfulness.

As already explained under the head of the moral imbecile, when a youth past this age of nine to ten still persists in these primitive practices, we are compelled to conclude that there has somehow been bad training. The primitive instincts have not been overcome, the new neuron paths have not been properly developed. This does not always mean that wise and good teachers and parents have not done the best they know; but rather, that in spite of themselves they have somehow made a mistake. 


\section{HABITS OF ACTION}

The first principle in dealing with these conditions is that the inherited neuron paths underlying immoral conduct must be blocked. Second, we must provide for the development of acquired paths.

Passing now to a consideration of general pedagogythe conscious help that should be given to the growing organism-we may state some general principles. We have already discussed at length the importance of experience. The child must be given those experiences that will set into activity all inherited neuron patterns that are useful, and also those that will develop his acquired neuron patterns in such a way as to be in the future most valuable to him. Suitable habits of action must be developed in reference to all the stimuli that the child is likely to meet. Rightly interpreted, this does away at once with a practice formerly considered wise, namely, that of keeping the child away from those experiences that are considered undesirable. The logical conclusion is, not that the child should not know about the things that we call evil, but rather that there should be developed in him the proper neuron patterns for right actions in the presence of these evils. To interpret by elaboration the prayer of Jesus, it would be, "Not that thou shouldst take them out of the world of evil, but that thou shouldst keep them from evil action."

The whole doctrine of the gradual growth and development of the nervous system; of the elaboration of neuron patterns as the child increases in age; the fact that the higher association centres do not develop until adolescence, and are not complete perhaps until the end of the adolescent period; all lead very clearly to the conclusion that we should not attempt to develop an appreciation of abstract moral principle or require the child 
to deal too much in abstractions until at least the beginning of adolescence.

When it comes to a consideration of school discipline or studies, an ideal procedure will first ascertain the mental level of the child, then the level of intelligence necessary to master each school subject and finally will fit the two together. It should be noted that while, on the one hand, a child of a lower mental level than that required for the understanding of a particular subject cannot possibly succeed in that line; on the other hand, it is equally an error to keep a child at work that is below his mental level. His instinet of self-assertion makes him rebel at this and he seeks by every device known to him to escape. When the subject will have received more scientific study than it has as yet, it is probable that we shall find that the truant group is largely made up of children who belong to one or the other of the two classes mentioned-namely: those whose mentality is below that required for the task and those whose mentality is far above the task.

Finally, in view of the facts of physiological and neurological development of the child, the question may be very seriously raised as to whether we are not, in our present practice, rushing him into abstractions too early and wasting his time by having him memorize things which he can only understand later; whereas we should be using the time in carefully developing neuron patterns of fundamental importance which are best and most easily acquired in the early years. If this question is to be answered in the affirmative it points most emphatically in the direction of the present tendency to industrial and occupational education.

The pre-adolescent years should be occcupied by the [292] 
child in doing things; in getting wide experience with the world about him; in getting thousands of neuron patterus in his sensory and motor centres, that will furnish the bases for elaborate thot, reason and judgment when his association neurons begin to develop and are ready to connect up these different experiences.

So much for the normal child. Now we must consider the feeble-minded, the backward, those who are arrested in the development of their neurons. In considering the pedagogy of the feeble-minded and backward child we must remember one very important fact, we are no longer dealing with a developing organism. The brain has ceased to grow and that stoppage has caught a great many of the neurons undeveloped and in such an im. mature condition that they will never be able to function. From what we know of brain growth and neuron develops ment; and from what we observe, on the mental side, of the condition of the intelligence in these individualsour studies have shown that the feeble-minded manifest all the simpler mental processes but are noticeably deficient in those that depend upon elaborate association -from all these data we conclude that the neurons most affected are located in the association areas.

The feeble-minded see and hear, taste and smell as do normal children. They meet and respond to a simple environment in a very natural childlike way. They profit by experience to the extent that they will repeat or refuse to repeat the experience according as it brot pleasure or displeasure, provided the second situation is identical with the first. They can neither generalize nor perceive similarities except those that are most obvious. The result is that a second situation, essentially like the first experience but presenting some different 
phases, is not recognized by the defective as similar, and consequently he does not know how to act. In general, it is the differences that he notes and not the similarities. Ask a child of eight why snow is like sugar and he may tell you that snow is cold and sugar is sweet. In what way are a butterfly and a fly alike, and he will tell you the butterfly is bigger. Even a child of nine or ten still sees differences more easily than likenesses.

Twenty-one boys from fourteen to forty-seven years old of mental ages 9,10 and 11 were asked to give the following likenesses and differences:

a. What is the difference between a cannon and a rifle?

b. In what way are a hat and a coat alike?

c. In what way are a hoe and a razor alike?

d. What is the difference between a hatchet and a hammer?

e. In what way are a rose, a potato and a tree alike?

f. In what way are a table, a chair and a bed alike?

g. What is the difference between a president and a king?

h. In what way are a cat, a snake, a bird and a fish alike?

i. In what way are the eye and the ear alike?

j. What is the difference between character and reputation?

The following table shows the result. 20 Bors

$\begin{array}{ccccc}\text { Mental } & \text { Average } & \text { No. } & \text { Likenesses } & \text { Differences } \\ \text { Age } & \text { Life Age } & \text { Cases } & \text { Correct } & \text { Correct } \\ \mathbf{9} & 20 & 4 & 21 \% & 44 \% \\ 10 & 25 & 11 & 38 \% & 59 \% \\ 11 & 25 & 5 & 73 \% & 75 \%\end{array}$

From this it will be seen that not until mental age 11 do we get practically as many likenesses as differences 


\section{SIMILARITIES AND DIFFERENCES}

recognized. This inability to see similarities has some curious results especially noticeable in moral conduct. A feeble-minded child of mentality 7 or 8 steals a handkerchief and gives it to somebody she likes very much. She is caught and corrected, told that it is stealing and is wrong. It is highly probable that she will never steal a handkerchief from that person again; but it is quite possible that she will steal something else from that same person or will steal a handkerchief from somebody else. She is not able to appreciate the generalization that it is wrong to steal, or even that it is wrong to steal from that particular person, or that it is wrong to steal a handkerchief from anybody. Because of her lack of neuron development, and the consequent impossibility of building up neuron patterns that would mean stealing is wrong, we cannot hope that she will ever grow out of her present condition. This has important bearings upon the problem of training.

Whenever a child cannot profit by experience, or has to be corrected a great many times for not doing right in situations that are similar to those he has experienced before, we may suspect feeble-mindedness or backwardness, or at least that the association neurons whereby he perceives similarities have not yet developed. This is in striking contrast to the usual normal child. The normal child of the proper age might manifest exactly the same state of mind; but, almost while we are correcting him and teaching him the moral principle, his neurons are developing and he is no longer the same child that he was when we began with him. He gradually develops the power of appreciating the abstract principle. Because his neurons are constantly growing, the possibilities of new patterns are just ahead. It is evident that 
the only thing to be done in the case of the feeble-minded is to teach them the right action in each particular case. Where one or two illustrations or one or two experiences would be sufficient for the normal child, the feeble-minded child must have scores.

When it comes to the question of formal education, this fact that the feeble-minded is not developing has another significant bearing. Many things that we teach the normal child require an ever increasing intelligence as we go farther into the process. But because the normal child is ever getting an increased intelligence, he is able to keep pace with the increasing difficulty of the subject. With the feeble-minded this is not true. Here is a subject or a process that requires, let us say, only 6 year intelligence to understand and manage the elements. But as we proceed with the subject, we quickly get to the place where it requires 7 year intelligence and then 8 and 9 and 10 . Here is a child of 7 year intelligence; he is able to begin the subject, but we know he will never get very far in it because he has only 7 year intelligence, and very soon the subject will require more intelligence than that, if it is to be comprehended. Shall we begin it? Certainly not, if the child is never going to be able to get far enough in it to be of any use.

This leads us to an extension of the principle that we laid down in considering the normal child. We must find out the amount of intelligence required for the problem in hand; we must ascertain the mental level of the child, and if his mental level is too low to begin the subject, it is obvious that we are wasting time in attempting to teach it to him. But more than that, if the problem in hand, altho in the beginning within his mental grasp, 


\section{TRAINING FOR THE FEEBLE-MINDED}

quickly develops to a point that requires intelligence greater than he possesses, it is useless to begin it unless it is of such a character that even an elementary knowledge, such as he can obtain, is useful.

From these general principles and considerations let us pass to more specific suggestions as to how to train the feeble-minded. It will be helpful to take them by grades beginning with the idiots. No one any longer thinks of trying to educate idiots; that has been sufficiently tried in the past to make it perfectly safe to accept as a fact that it cannot be done. Practical experience has formulated as the limit to be striven for in his training, the following: "To try to fix upon him a few simple habits so that he will eat a little less like an animal and make known his physical wants to the end that he may be kept clean and comfortable." Even this is unattainable in the lowest grade.

The low grade imbecile can be taught to do all the things just mentioned, to amuse himself with simple play, to take some care of himself, such as buttoning his clothing, lacing his shoes; and to do very simple errands in the sight of the parent or teacher.

There is a gradual development thru the middle grades up to the high grade imbecile, who can, with patience, be trained to do some work of simple character or to participate in more important work by doing the simpler parts of it. But everything must be concrete and definite, the instructions must be explicit, and the trainer must expect a great many failures and mistakes until finally a definite neuron pattern is established. With careful training, long persisted in, a person of this level of mentality may learn to wash dishes, scrub floors, dust furniture, make beds, dig dirt, pile stones, do special er- 
rands for which he has been especially trained and under circumstances where the conditions will not vary from time to time. But the moment variations occur the child is helpless.

The low grade moron is of ten found doing many of the things just enumerated, but he learns them more quickly and easily, is more reliable and can adjust himself to slight variations. He will perform longer tasks and somewhat more complicated ones, and with sufficiently long and painstaking training can do quite a good deal of work about the house or on the farm, provided he is constantly watched and told how to meet the situations that vary a little from the usual.

Finally, the high grade moron ean be trained to do almost any kind of work that does not require special skill, provided again, the training be carefully and painstakingly and persistently carried on until the process, whatever it is, is learned. He is able to adapt himself somewhat better to changed conditions and will often meet new situations fairly well-if the new situation does not involve too much that is not within his direct experience. For the things in which he has been trained he needs only occasional supervision. His great lack is that he cannot plan. High grade morons can be trained to be excellent assistants in a great many lines of work; such as assistant janitors, assistant cooks, assistant painters, and so on thru a long list.

In fact, it is now known as we have already shown, that there are a great many men and women that work in these various occupations who are mentally only morons. They have, by lucky chance, fallen in with some one who was able to train them; and once trained, they go on very well until some situation arises for which their 


\section{FIRST PRINCIPLES}

training has not been specific and which requires judgment. For instance, Eddie has learned to handle a paint brush and can paint a plain surface or varnish a floor in a reasonably satisfactory manner, so much so, that he is useful in that eapacity. But if set to paint the floor of a room that had only one entrance he would be quite apt to begin at the entrance and paint away from it, so that when he was thru he could not get out without walking over the wet paint.

These facts, which have been found out by those who have spent years in the training of feeble-minded children in institutions, point to some very definite lines of action for those who have to train such children either in institutions or in public schools, or in the home. First, as we have already insisted, the grade of mentality of the child must be discovered; then certain tasks that are within his reach must be definitely selected and put before him and he must be carefully and persistently and patiently trained to do them. There are many things to be done in every community that require no more mentality than that of a middle grade imbecile. A decision must be made as to which of these things shall be selected. This will depend partly upon the temperament of the child in addition to his mentality and partly upon the environment in which he lives. The occupation selected should be such as fits any special interest or ability he may have, and secondly such as he is likely to have an opportunity to work at, in order to earn such wages as he is capable of earning.

We may generalize by saying that beginning with the younger and lower grade children the training should be: first, in the direction of self-help; then in the direction of helping others; and lastly of doing things that will 
enable them to earn so much of their living as is possible. Now the first of this concerns naturally their care of themselves. Every child who, for any reason, does not learn to keep himself clean must be taught it. If he does not learn it at home it is a fitting subject for school training. First, to keep his body clean, by washing hands and face, and by bathing; secondly, to care for his clothing, both in the way of cleanliness and in the way of simple mending. While the latter suggests training for girls, it is equally fitting for boys. They may quite well learn to sew on buttons, to sew up rents in clothing and to do other simple work.

From this care of themselves we next proceed to care of their surroundings. Scrubbing floors, sweeping, dusting, even washing windows and washing clothes are all suitable subjects for the school curriculum for the feebleminded. We may pause to call attention to the fact, which may be overlooked, that all this is not only practical and useful, but it is mental training of the best possible sort for these children. It is giving them definite concrete experience; it is developing neuron patterns that will always be useful.

Next to cleanliness of themselves and their surroundings is training in the simpler activities of life. Here comes in the preparing of meals; setting of table, washing the dishes, table manners, such preparation of the food itself as can be trusted to them; even simple cooking. To this end, every special school should have a kitchen where may be done all the work that is done in the homes of these children. Then there is the provision for sleeping. The care of the bedroom and the bed, the washing of the bed linen, mending it, et cetera.

Next comes the out-of-doors. A certain amount of [300] 


\section{SPECIAL TRAINING}

farm work or at least garden work may properly be taught to all. Farm work is the great opportunity for these children. They like it and can do much under supervision. Those who live and are likely to live in cities may soon be turned in the direction of such occupations as those at which they are likely to be employed. This brings us to a large topic; that of training them in some work whereby they can help to earn their living.

Here again we must clearly appreciate the difference between the normal and the feeble-minded boy or girl of working age. The normal boy or girl may go into a factory, secure a job, be set to work by the foreman, who shows what is to be done and how to do it, and in the course of a few hours, or at most a couple of days, has mastered the job and is earning his wages-a permanent employé. On the contrary, the feeble-minded boy or girl cannot do this. He does not learn the job the first day, nor the second, probably not the first week. The result is that at the end of the week he is dismissed. No foreman has time to put up with such "inefficiency." Now the only way this situation can be met is for the children to be trained in the schools to do these things. In other words, ordinary factory work, piece work, is to the feeble-minded boy or girl what the skilled mechanic's work is to him. It requires careful training, long and painstakingly worked out. A simple experiment made in the Vineland Laboratory will illustrate this.

The Bogardus Factory Test consists of a board about two feet square, in the centre of which is a standard from which two arms rotate. These have on the ends of them pieces of steel to simulate knives. A pair of inch cubes are provided; a square inch is painted on the board in 


\section{PSYCHOLOGY OF THE NORMAL AND SUBNORMAL}

the path of the knives. The knives are rotated by a motor at a suitable speed and the person is required to take one of the cubes in his right hand, place it on the square, pick up the other cube with his left hand, transfer it to the right hand, and when the knife knocks the first cube off the square, place the second cube on the square, pick up with the left hand the one that was knocked off, transfer it to the right hand and so on. The requirement each time is to place the cube on the square and get the hand out of the way without getting hit. The whole procedure is intended to simulate a good many factory operations. Most normal people do the test at once. A few require a little time to get the rhythm and the new muscular co-ordination which enables them to shift the block from one hand to the other and place it on the square at the right moment so as not to get hit. Four moron girls of ages ranging from sixteen to twentytwo were practised on this apparatus for about a half an hour a day, with the result that an entire week passed before any of them was able to do it successfully. It is easy to see that any employer who was paying these girls wages for that kind of work would have discharged them at the end of the week if not before. And yet, having once learned this process, they could do it as easily and successfully as any one else. The point is, they needed to be trained to do a simple task which the normal person can do without any training. This is a maxim worth remembering in the training of the feeble-minded. They must be trained to do things that the normal child does without ever having been taught, without knowing he has learned to do them.

In all this we have said nothing about the usual school work, reading, writing and numbers. It is evident from 


\section{READING}

what we said several pages back that only the very highest grades are to be considered for these subjects at all. The others either have not intelligence enough to begin, or their mentality is such that they will never get far enough in these subjects to make it worth while for them to begin. A certain number of high grade morons can be taught to read and write and make use of simple numbers, altho always at great expense of time and effort; but of those who can be thus taught, very few ever make any real use of such accomplishments afterwards. Every institution for the feeble-minded has abundant opportunity to investigate this problem.

Practically all the morons who enter such an institution have been trained in these subjects, either in school or at home, and usually come with the statement that they can read and write and do elementary arithmetic. Miss Lindley (31) recently made an investigation of their reading with this result: Of 108 moron girls and boys who had been taught to read and who declared they could read, only 10 were found to ever actually make any use of reading; and many of those who said they could read and probably had once been able to read a little, were totally unable to read anything except a few of the commonest words.

The following is an abstract of Miss Lindley's report:

\begin{tabular}{ccccccc} 
Mental Age & \multicolumn{3}{c}{ No. Investigated } & \multicolumn{3}{c}{ Readers } \\
VIII & Boys & Girls & Total & Boys & Girls & Total \\
IX & 28 & 10 & 38 & 1 & 1 & 2 \\
X & 22 & 9 & 31 & 5 & 2 & 7 \\
XI & 16 & 12 & 28 & 8 & 4 & 12 \\
Grand Totals & $\mathbf{4 0}$ & 7 & 11 & 3 & 4 & 7 \\
\hline
\end{tabular}

It was first made certain that all children upon whom [303] 


\section{PSYCHOLOGY OF THE NORMAL AND SUBNORMAL}

the investigation was based had been taught to read, and had at some time read fairly well.

If we select from the list of those that do read the ones who do so voluntarily and in preference to doing other things, we have but seven boys and three girls.

Those children who are easily disturbed seem to find reading too exciting. One boy said in substance that he is afraid to read since he had had a "spell." A girl of this type became so excited over The Shepherd of the Hills, a book that she had read again and again, that she tore it. Another girl burst into tears whenever she came upon a word that suggested one of her many complexes.

It is interesting to find that a number sit with books and pretend to read; also that many who can read very well prefer to be read to.

One may be very easily deceived in respect to the children's reading if their word is taken regarding it. They tell many exaggerated stories of the amount of reading they do. There is, as a rule, a tendency to pretence that is quite amusing. Most of those who cannot read are loth to admit it. A boy thirty-eight years old, with a mentality of 4 , sits for hours with a book and pretends to read. As a matter of fact, he cannot read a word. One day, after being called three times to come and be shaved, he slammed the book and grumbled disgustedly, exclaiming, "John can't read!"

The following conversations illustrate the pride felt in regard to being able to read and the subterfuge resorted to. A boy thirty-eight, mentally 8 , cannot read at all. Yet this is the conversation verbatim:

E. "Charley, do you read?"

C. "Oh, yes. I'm a great reader."

[304] 


\section{READING}

E. "What kind of books do you like best?"

C. "'Tain't no difference, just so there is readin' in them."

E. "Which do you like better, to read for yourself or have some one read to you!"

C. "Oh, I'd rather read to myself. I like to go off in the corner and stand and read for an hour or two at a time."

Here the examiner began to wonder if after all Charley could read, so taking a letter from her pocket she showed him the envelope and said, "Read this to me, Charley."

C. "Well (scratching his head), that's pretty hard."

$\mathrm{He}$ examined it closely for quite a while, then his face suddenly brightened and he said, "Oh, I know that; that's care of the Training School."

He had evidently learned the sign for "Care of."

E. "Fine, Charley. Now read the line above."

C. "Oh, that's your name, and I don't know your name."

E. "Of course not, Charley. That isn't fair, is it? But here is some printing on the back of the envelope. Read that."

C. "I could, but it's too fine and I can't see it very well."

Gilbert, twenty-seven years, mental age 7, who can read little, if at all, reports daily on current events, saying that he gets his information from the newspapers. $\mathrm{He}$ is much interested in the war, and here is a report of one of his conversations:

E. "Well, Gilbert, what's the news from the front today?"

G. 'The war's getting worse. Hollum's goin' in now and take it." 


\section{PSYCHOLOGY OF THE NORMAL AND SUBNORMAL}

\section{E. "Take what?"}

G. "The war. She's got a big army."

E. "Yes, but so has Germany."

G. "I know, but Germany's on her last feet. She's got submarines and she can't use them."

E. "Why can't she?"

G. "She can't keep them up."

E. "How's that?"

G. "'Cause she can't get the power."

E. "What about the United States?"

G. "We got awful good guns. If we go in we can help a lot. We got machine guns that shoot nine miles and the bullets go twelve yards."

James is eight years mentally and has never learned to read, yet here is what he says about it:

E. "James, can you read?"

J. "Oh, yes."

E. "What do you like to read best?"

J. "First readers, second readers, third readers."

E. "Take this paper and read to me."

J. "I can't read journals, only books."

E. "Read this line for me, it's quite easy."

J. (Examining it closely.) "I can't read that 'cause the letters are different in papers than in books. Books is what I read."

In strong contrast to this pride in reading is one boy who can read quite well, but says he never does because "only sissy-boys read, and I'm a man now and I'm not going to be a sissy-boy any more."

This investigation has been based upon 108 subjects who have painstakingly, and often with the greatest diffculty, been taught to read. Twenty-eight, or about 25 


\section{MISS LINDLEY QUOTED}

per cent. still read to some extent, while but 9 per cent. care enough for it to do any amount of it voluntarily.

There are no noticeable sex differences. Boys from the same kind of homes and with the same sort of environment here seem to read fully as much and as difficult material as do the girls.

Not more than two or three do reading more difficult than that of the fifth grade. Most of it is very simple, and the average would not be beyond third grade.

No children of seven-year mentality seem to read. While there are two eight-year-old readers, one is a psychopathic case and has, without doubt, tested much higher. Twenty-three per cent. of nine-year-olds and 42 per cent. of ten-year-olds read. There are so few cases in the eleven-year group that it is scarcely fair to consider them as representative. However, out of twentyeight readers, nineteen, or nearly 68 per cent., are found in the ten-year and eleven-year groups. Such results seem to warrant the conclusion that these are the only mental ages that will ever get much from knowing how to read. It is, we feel, these children who should have the attention, while with those whose mental development will evidently stop before this age, it is useless to spend time and labour. With our present knowledge regarding the defective child it is possible in most cases to make early, and rather accurate prognoses, and it is not necessary to continue wasting time with them. This is a point that is just as valuable for the Special Class teacher as it is for those who work with the child in an average institution. If the child is to remain at large it may be of advantage to him to do simple reading, but the results here seem to show that if he has that knowledge he is not likely to be able to use it to any real advantage. 
$\mathrm{He}$ is much more likely to use it to his disadvantage, for, as we have seen, his taste runs to the things that are not good for him. Besides, here as everywhere else a little knowledge may easily prove to be a dangerous thing.

Those of us who have laboured in vain to teach the defective child to read may find some comfort in the fact that so few of those who have learned, derive either pleasure or benefit from the accomplishment, and we may give with a clear conscience our time to things that for him are much more worth while!

Writing is in even a worse state. We have given elsewhere (20a) samples of the handwriting of the high grade feeble-minded. In institutions a few more feeble-minded children can write than actually read because of the constant pressure to "write home." But if they were left to themselves, very few letters would ever be written home and very little use ever made of the ability to write. The penmanship of the feeble-minded has peculiarities easily recognizable by any one familiar with their writing. This is, of course, to be expected since penmanship requires fine co-ordinations that are usually lacking in defectives. This lack of co-ordination resulting in angular, irregular lines is quite a different thing from the careless or hasty writing of a business man whose letter may be hard to decipher.

In number work the story is quite similar. We have shown elsewhere (20c) feeble-minded children "rarely if ever develop a true number concept." Because of their good natural retentiveness they can memorize certain combinations and processes-addition, subtraction, multiplication. I have never known one to master long division. Written problems involving anything more than 


\section{WRITING AND ARITHMETIC}

simple addition or subtraction are quite beyond their abilities-except in school where they may have memorized the problem and its solution.

The fact is, of course, that all these subjects are highly abstract, they involve elaborate associations and neuron patterns that, if not utterly impossible for the feebleminded, at least are made with very great effort. It is probably easier to make these children happy and useful in some other way than by trying to teach them reading, writing and counting. But one thing is evident, if these subjects are to be taught them they should come as extensions of their other work. There should be just such use made of these accomplishments as the house-wife or the farmer boy makes. Making memoranda of household articles to be purchased; reading and writing the names of the things they work with; counting the number of people, the number of plates, horses, loads of coal, and what not; perhaps simple measurements of things they work with. Moreover, in this way will be discovered most easily what the child is capable of in these lines. Those that show some capacity may be helped according to the judgment of the teacher. Those who are not able to learn a little reading and writing and numbers in connection with their daily work, will be found incapable of receiving any more elaborate instruction in these lines. 


\section{CHAPTER VII}

\section{MORAL TRAINING}

THe necessity for definite moral training of the developing mind lies in the fact, already discussed at length, that the child is born with inherited neuron patterns which lead to impulsive and instinctive actions. While adapted to man's welfare in a more primitive condition of society, many of these actions are no longer tolerable because of changed conditions and especially because of the development of the gregarious instinct and the habit of living in co-operative societies. Consequently the neuron patterns must be modified.

In attacking the problem of moral training there are three questions to be answered, three groups of facts that must be understood. First, what is the nature of the child who is thus to be trained to a different habit of action than that which his inborn capacities would naturally lead to? Second, what kind of training do we wish to substitute, in other words, what is our ideal? Third, what are the means by which these results can be brot about?

It is well recognized (and everything we have said in this book emphasizes the consideration) that moral training must begin early. As soon as the child is born he begins to react to his environment and as we have already seen, the action tends to become habitual. All those actions that will be useful may be allowed to take care of themselves; altho conscious appreciation of the value of these actions may result in their being more quickly re- 


\section{NATURE OF THE CHILD}

duced to habit and more securely established. But we are more especially concerned with those natural reactions which, if not corrected, would lead to habits that would be of decided disadvantage both to the individual and to society. Hence, moral training cannot begin too early. We proceed then to consider our three problems in order.

Nature of the child. We have only to recall that the child is a growing and developing organism to realize that our moral training must be gradually applied and must be adapted to the child's stage of development. In other words, the time to modify an undesirable natural reaction is when that reaction takes place, and not when the teacher or parent arbitrarily decides that it is time for the child to develop this new accomplishment. With the normal child this is not such a difficult matter for the intelligent trainer.

The age of the child and his physical development are a rough but fairly satisfactory guide to the thotful parent. One is not apt to try to impress ten year old conduct upon a two year old child. Nevertheless both parents and teachers often err in following this guide. Many are ambitious and want to force the child into conduct that is beyond his capacity to appreciate. We are as a rule ignorant of the capacity of children at various ages. We are prone to interpret the actions of the child in terms of our own experience and to asscribe to him motives, intelligence, and responsibility which he does not possess. Others go to the opposite extreme and, blinded by love and affection, excuse and pass over conduct which should be corrected.

Besides the age and physical development, we have the fact that the normal child is active; constantly doing 
something. This is helpful because it shows to the intelligent disciplinarian the stage of development that the child has reached. All these facts render the training of the normal child more or less difficult; but the problem, tho requiring thot and intelligence, is nevertheless not an insolvable one, as is evidenced by the fact that most parents who devote themselves definitely and consciously to the training of their children achieve a reasonable, and often a high, degree of success.

It is a dull, backward, or feeble-minded child that presents the great problem. Here his age and physical growth deceive us, and his lack of activity deprives us of very sure indications of the state of his natural impulses. In such cases the nature of the child can only be made out by most careful and wise psychological study. We must ascertain by careful tests the mental development of the child and adapt our treatment to his mental level and not to his chronological age or physical condition. We should ever bear in mind the facts of neuron development and neuron patterns as set forth in the earlier chapters of this book; and remember that it is futile to attempt to develop a habit of action, the neurons for which are not yet full grown. The difficulty of carrying out this principle increases directly as the chronological age and inversely as the mental age. By that we mean that the older and larger the child grows, the more apt we are to insist that he is big enough and old enough to carry out a prescribed line of conduct, unless his mental age is extremely low, in which case we readily recognize the condition and excuse him. We recognize the idiots and low grade imbeciles and do not hold them responsible for conduct that is befitting a six, eight or ten year old child. When, however, the mental 


\section{THE IDEAL}

age of the child is higher than that of the low grade or middle grade imbecile, the difficulty becomes correspondingly greater, partly because we do not recognize that he is backward, and partly because we do not know accurately what is the proper conduct for children of various ages.

When we come to the moron grade we have the great problem, as yet unappreciated by the masses of parents and teachers. A sixteen year old boy with a mentality of from 10 to 12 is not recognized as defective by any but experts. His conduct is attributed to carelessness, indifference, indolence, pure wickedness, or natural depravity. In such cases we are apt to treat the symptoms rather than the underlying conditions. While it would be folly to maintain that every youth who does not manifest conduct becoming his chronological age is mentally defective, yet we now know that this is the true explanation in such a large proportion of cases that it is by all odds the wisest procedure to suspect mental defect until the contrary is proved. An entire change of attitude on the part of parents and society in general in this respect would result in an enormous simplification of our problems of delinquency, not only among the youth but among the so-called adults-adults in years but often children in mind. To go on treating as responsible individuals, once the facts are known, persons who are arrested in development and have only the mentality of children with the consequent child's responsibility, is to ignore the dictates of common sense.

The Ideal. Strangely enough our second problem, the ideal, or what kind of training we wish to give and what we wish to accomplish, is probably the least definite of the three problems. Earl Barnes has said that if we 
could agree upon a body of doctrine that we wished to teach children, we could transform the nation in a generation. This is perfectly true, but the fact is we do not agree, at least we cannot get beyond glaring generalizations. We are agreed that we want to make "good and useful citizens," "honest," "truthful," "'humane," "efficient," etc., etc., but when it comes to the definition of these qualities we are widely divergent.

We can only say that from the psychological standpoint our ideal is, as we have stated in the chapter on habit, to fix in the lower nerve centres as large a. group of habitual actions as possible, and such a group as will insure the individual's acting in a way that will be most useful to himself and to society. This will include something of the virtues above mentioned and many others. It has all been summed up in the expression, "preparation for life." With the normal child this means, of course, preparation for an independent existence; that is to say, the ability to manage his own affairs, to take his place in the world, to provide for himself and to contribute something to the general welfare. With the feeble-minded, however, the case is again different. With all but a very few of the highest grade, it is useless to have such an ideal as just expressed. We cannot prepare them for a life of independent action. They cannot provide for themselves, much less contributs anything to the general welfare. Consequently, our ideal must be to so train them that they will be as little a menace to, and as little a burden upon, society as possible, and, in their own life, as happy as possible.

Method. Passing to our third problem we have to consider the question, how is our ideal to be accomplished. We have shown in the previous chapters how the instincts 


\section{MOTIVE}

are to be modified, neuron patterns formed, experience given, so that the individual has all the necessary mechanism for reason and judgment and thot, and the neuron patterns underlying right action So far, however, we have said nothing about motive. We have said that all the nerve mechanism and all the mental processes have for their sole purpose the accomplishment of an act; but what is the purpose of the act? If it has no purpose then there is no need of modification. The nervous machine is what it is, the stimuli produce certain results in the form of actions, and nothing more is to be said. Mere actions, whether impulsive, instinctive or reflex, involve no problem such as we are now discussing. There is no need for modification of the instinct or for control.

But the moment the action which results from any nerve activity has to fit in with other actions and correlate with a larger whole, that moment we begin to have a problem of adjustment. The intelligent adult, realizing the problem, proceeds to make the necessary adjustments in accordance with his experience, judgment and reason. But unfortunately by the time man reaches this stage he has already formed a vast number of habits of action, many of which would be exceedingly detrimental to his adult ideals. Consequently we conclude that it is a kindness to the adult of the next generation for the present adults, with their experiences and ideals, to exercise a control and a directing influence over the development of immature mind.

Persons of undeveloped mind not being sufficiently intelligent to have ideals for themselves, intelligent adults must have ideals for them and must see that the development is in accordance with those ideals. But experience 


\section{PSYCHOLOGY OF THE NORMAL AND SUBNORMAL}

quickly teaches that it is one thing to have ideals for the child, and it is quite another thing to induce him to follow those ideals. Experience also teaches us that a cardinal principle of all training of children is: first to secure their co-operation.

This brings us to the fundamental problem of discipline; and the question comes, how shall we secure the cooperation of the child in our efforts to bring about the necessary modifications of such of his natural instincts as experience has taught must be modified. Fortunately the answer is at hand, at least, in general terms.

Biology teaches us that evolution, or development, in both the plant and animal world, has come about thru a system of rewards and punishments. In unconscious organisms this is simply life or death, survival or nonsurvival. That organism that varied in such a way as to best adapt it to its environment survived; those that were not thus favoured perished. The reward is life and the punishment death. With conscious organisms and especially with man there is a wider range of possibilities thru an appreciation of the tendencies of actions that in themselves are not of sufficient importance to result in a question of life or death. Thru the functioning of the sympathetic system, feelings arise.

These feelings serve as guides for future action. If they are pleasant they encourage the activity that gave rise to them; if unpleasant they discourage it. This gives rise to a natural system of rewards and punishments. This system has been efficient in the evolution of the race, but it is costly for the individual. The rewards and punishments are often so remote that the individual cannot appreciate them, and consequently does not attempt to modify his conduct until it is too late.

[316] 


\section{REWARDS AND PUNISHMENTS}

The next generation will profit by his experience, but he is lost. To obviate this we have developed a system of artificial rewards and punishments. It is these and their application that we have especially to consider.

By punishment is meant anything that causes pain or discomfort, unhappiness or loss. Reward is anything that gives happiness, pleasure, or gain. There is an ascending scale of both rewards and punishments. The lowest form is that which appeals mainly or solely to the physical, bodily pain or bodily comforts. At the upper end we have the highly abstract form of subjective happiness or unhappiness. Obviously the lower forms are applicable to the earlier stages of development and the higher forms only to the later stages.

A question often raised is, which is preferable, the discipline of rewards or the discipline of punishments. Since the two are often reciprocal it is in many cases only a question of emphasis. In practice it has come about, in the past at least, that we have laid the emphasis on punishments in the lower levels and on rewards in the higher. We punish a child for wrong conduct; we reward an adult for good conduct. There is, however, an encouraging tendency in later years to lay greater stress upon the positive side with a consequent greater use of reward and less of punishment. Formerly trainers of animals punished the animal when he did not do the thing that was wanted. Animal trainers have, however, learned that better and quicker results are obtained by rewarding the animal when he does the right thing. The reason for this seems obvious, once one's attention is called to it. There are innumerable wrong actions for every one that is right. Consequently the chances are far greater that an animal will do the wrong thing than 
the right. If we are using the punishment method we will be continually punishing him; and as a consequence numerous associations are made with the wrong reaction and none with the right one.

Moreover, we have learned that the emotional content of an experience has much to do with the strength of the association; therefore, punishment for an action makes the neuron pattern underlying that action all the more strongly affected and more likely to be used again. On the other hand, when all the wrong actions are ignored they are easily forgotten, and then if the right action is rewarded it becomes the one association that remains fixed in the mind. There seems to be no reason why this is not as true of children as of animals. Indeed there are reasons to think that it is more important. Punishment puts the child into an unhappy frame of mind which in many cases seems to make it impossible for him to conceive the right action; or even if he does finally act right and gets rewarded, the reward is insignificant in comparison with several punishments. Moreover, it is a demonstrated fact that punishment takes away energy, while reward increases it. Gilchrist (19) tried the following experiment:

A class of fifty young women in educational psychology were given the Curtis English Test 4 B. Immediately after taking the test, the class was divided at random into two groups and seated in different rooms. To the first group the examiner said, "A hasty examination of the papers in the test just given shows that the members of this group did not do so well in the test as the average twelve-year-old child would do. I ask you to take the test again." To the other group he said, "A hasty examination of the papers in the test just given shows that

[318] 
the members of this group did exceptionally well. I ask you to take the test again." The first group made no improvement on the second test, while the second group improved seventy-nine per cent.

The writer has repeatedly made the experiment of speaking encouragingly or discouragingly to subjects who were using the ergograph. The results were always the same; the subject always lifts the weight higher when praised, and he always falls below when he is criticized. Say to a bystander, but so that the subject hears it, "He is making a fine curve," and the next pull goes higher than any he has made. Then say, "Pretty poor," and the next stroke will be lower than those each side of it. These and many other considerations certainly justify the rule, never scold. We cannot, however, go quite so far as to say never punish; tho it is undoubtedly true that on the whole vastly better results are obtained by the method of reward than by that of punishment. But it should always be remembered that it takes the highest wisdom to punish wisely. A fundamental principle in all punishment is that the form and degree of punishment must fit the offence to a nicety. As much harm is done by overpunishing as by not punishing at all. The psychology of punishment should always be kept in mind. The purpose, of course, is to associate such unpleasant consequences with an undesirable action that that line will be blocked in the future. In view of this it is obvious that the punishment should not be too far removed in time, from the offence; otherwise the child makes no association between the two. A punishment that has no direct connection in the mind of the child, with his offence, is not punishment but brutality.

Rewards have the advantage, as already indicated, of 
increasing the energy, strengthening the association, and encouraging the highest degree of co-operation on the part of the subject. The reward must be carefully selected and, like the punishment, wisely adapted to the action. It also must be used sufficiently close to the action to make the association complete, otherwise it will have no effect upon action. By reward, we do not mean bribe. While a child may occasionally be promised a reward, as a rule the reward should follow the action without previous promise. The action performed, the reward should be forthcoming, and should not be withheld on account of any later misdemeanour. Punishing a misdemeanour by withholding the reward for a previous good act produces a confusion in the mind of the child, and neither the reward nor the punishment gets its proper associations. Give the reward as deserved for the thing done; punish the later misdemeanour on its own account.

With very young children the reward must be definite, concrete and prompt. The one thing that often fulfils all these conditions is something to eat. This appeals to a fundamental instinct and makes a strong association. It is the procedure now followed in the training of animals, as above indicated, and wonderful results are achieved by it. To promise a young child something in the future is absolutely futile. We have already shown that a child does not know the difference between morning and afternoon until he is six years old. Therefore, to promise a young child something "this afternoon" or "tomorrow morning" is meaningless to him. Similarly, things more remote are equally useless to an older child. To promise something next month or next spring to a child who has not yet arrived at the stage where he appreciates "the date" is equally useless; while to hold [320] 


\section{USE OF COMMENDATION}

out to the child the idea that a certain line of conduct will make him "a respected man when he grows up," is undoubtedly utterly lost any time previous to the beginning of adolescence.

The writer has tried the experiment a number of times and has never found a school group that would not choose a half-holiday "today" in preference to a whole holiday next week. There are probably other factors present in such a test, but undoubtedly the one under discussion is prominent. The food reward, which is the only proper one for the very youngest children, may, as the child grows older, be replaced by things of value to him. Still later may come in the appeal to the self-assertion instinct, the showing off, in the form of honour, rank, position, or privilege. Under this head come also words of approval or praise, especially if made in public.

Commendation and approval given to the child in private should be freely bestowed when he has reached the age to appreciate it; but this probably comes rather late, certainly does not reach its highest efficiency until the adolescent period or even adult life. Lastly that "virtue is its own reward," or that a person should be satisfied with the consciousness of having done right, has no place with children and not often even with adults. It may perhaps serve for a time with some persons of highly evolved consciousness for abstract ideals; but every normal man wants the approval of his fellows and must occasionally, at least, hear the word of commendation; and most men are more efficient if this commendation comes pretty frequently. The most successful leaders of men are those who see most to praise in their followers.

It is sometimes feared that too much praise makes [321] 
the child conceited. This is probably not true, but even if so, the evil is far less than that which comes from lack of appreciation. Praise brings out the best that is in a child, while censure or disparagement discourages him from attempting what he really could do.

What we have said of normal children is also true of the feeble-minded according to their mental age rather than their chronological. But more than that, because of the lack that we have described in the feeble-minded, they are unable to formulate for themselves any ideals and consequently the only incentive to action is the rewards that they get, either material ones or, in the highest cases, the word of public commendation. Without working it out psychologically, the caretakers of the feeble-minded have learned empirically that the most successful way of handling them is to constantly praise them. It is found that to praise a child even for a piece of work that is not quite satisfactory is the surest way to make him do it better the next time.

We have seen that participation of the sympathetic system in our nervous activities seems to reinforce the action of the central neurons and when the affective element is strong, the experience is more vivid in consciousness, and is better remembered, and the different parts of the neuron pattern are more firmly linked together. It would follow that when the interest phase of the attention-interest state-is so strong as to be noticeable, the effect is better. This is borne out by the common experience that interesting experiences make life more worth living; make the body function better; conduce to health and long life.

This conclusion is abundantly reinforced from the study of the feeble-minded. The motto of a certain 
school for the feeble-minded is "Happiness first, all else follows." This is not a philosophical dictum but an empirical formula, the result of long experience in the care, training and study of mental defectives. It means that when the child is so treated that its sympathetic system augments the action of the cerebrospinal system by that something, which shows in consciousness as pleasant emotion, interest, or happiness, the resulting action of the entire system is more nearly normal, more satisfactory than under the opposite conditions.

Children learn better, work better, act better when they are happy. Ever since this principle began to be appreciated, the institution has been organized for happiness. If a new plan or a new policy or some new arrangement is under consideration, the first question asked is, "Will it make the children happy?" If a child is under consideration, the first question is, "Is he happy, or will the treatment proposed make him happy?" This does not mean a soft pedagogy or weak discipline, nor that the child gets necessarily what his momentary whim leads him to think he wants. But neither does it mean that some matron or teacher or superintendent in his superior wisdom decides that this thing, in the long run, will make the child happy and therefore he must submit to it, trusting that he will live long enough to enjoy the happiness and appreciate the wisdom of the treatment.

"Happiness first" means that the nature of the child is considered, his temperament and disposition and habits; that the treatment best suited to him is prescribed; and then by careful handling he is gently led away from the thing that he thinks he wants, and his interest is aroused in the thing that will actually make him happy. That this is a real and definite achievement 
will be appreciated by any one who knows of feebleminded children that are not thus treated; who knows how cross and stubborn and ugly, out of sorts and uncomfortable and irritating, feeble-minded people can become. Indeed, those who know feeble-minded children as they are usually met with, handled by those who do not understand them, find it hard to believe that children in an institution for the feeble-minded are happy. Nevertheless, it is true; and every institution that works upon this principle of happiness, as most of them do, contains one of the happiest groups of people that can be found.

The children in institutions are fundamentally like the defectives outside. They have their bad ways and their bad days, their bad dispositions, their annoyances and vexations, their unhappy moments; but it is the business of the first one that comes in contact with them to change all that and make them happy. Let us see how it is done.

John, age thirty-five, mentally 7 , is one of the farmer boys, he has been carefully trained and can plough and harrow and drive a team and do various other work when once it is planned out for him. One morning he came over to the stable and the farmer said to him, "John, you can plough that field today," but John was not feeling happy. Perhaps he had a bit of indigestion, or he had not slept well. He did not appear with his usual good nature, and as a consequence he replied to the farmer, "Ain't goin' to plough that field today." To which the farmer replied pleasantly, "Not going to plough the field, what do you want to do?" "Nothing." "Nothing? well, all right, do you want to go back to the cottage and rest today?" "I don't know, I don't know, 


\section{MAKING A MORON HAPPY}

I don't want to do nothing." “All right, John, you just do nothing today. You just have a good time doing nothing. I'll find somebody to plough the field and you can do nothing all day. Of course, I am sorry that you don't feel like ploughing the field, because while I can find somebody to plough it, there isn't anybody that can plough it quite so nicely as you can." And the farmer worked about, pretending to do something but in reality waiting for John. John could not withstand that little bit of flattery-any more than a normal person. The fact is, it made him happy and the moment he was happy the whole situation changed. After a few minutes he came along, perhaps rather sheepishly and said, "Well, I guess I'll plough the field." He got the thing that made him happy.

Tom is a high grade moron. He has been trained to use the paint brush. There are two painters, one of whom he likes very much but the other one irritates him. It happened that his friend had gone for a short vacation, so that Tom had to work with the painter whom he dislikes. One day he came to the administration building very angry and excited and wanted to see the superintendent. The superintendent was busy with the director of the laboratory, talking over important matters, but these matters could wait. Anything can wait when the happiness of a feeble-minded boy is at stake! Tom came in and, almost crying with anger, he shouted, "Ain't going to work with Jim any more. I want you to give me a note saying that I don't have to work with Jim." "All right, Tom, but tell me what is the matter." Tom explained his grievances as well as he could, he said he liked to paint and he would paint when his friend came back, he would work with him but he would not 


\section{PSYCHOLOGY OF THE NORMAL AND SUBNORMAL}

work with Jim. The superintendent proceeded to write out the order for his transfer to another line of work. Tom was gradually getting over his anger. He seemed to be getting what he wanted, what would make him happy. Presently he said, "I can come back to the painting when my friend comes back, can't I?" To which the superintendent replied, "Well, now I don't know about that, Tom. You see there is a lot of painting to be done, and I must have boys working with $\mathrm{Mr}$. Blank who will be willing to take his place and work with Jim when Blank goes away. He has to have a vacation, you know. You wouldn't want him to stay home from his vacation, would you?" "No, I wouldn't want that, but I want to work with him when he comes back." "Well, you see how it is, I must have boys working with him when he is here who will be willing to work with Jim when Mr. Blank is away." And the superintendent continued to write the order. I could see that Tom was thinking hard. Presently he said, "Well you needn't write the order, I'll go back and work with Jim." He got what he wanted and was happy. Not that he wanted to work with Jim, but he wanted to work with Mr. Blank and the smaller thing is swallowed up in the greater.

"Happiness first, all else follows," is surely based upon sound, scientific facts-the physiology of the sympathetic nervous system. Perhaps the reader will ask, what is to be done with those persons, normal or defective, who cannot be made happy. To which we shall reply briefly; that is a problem for the physician, not for the psychologist. In other words, the person who cannot be made happy is diseased and it is for the physician to discover the disease, its proper treatment and its cure.

One might sum up the problem of discipline among [326] 


\section{MAKING A MORON HAPPY}

the feeble-minded by saying:-Treat them as children according to their mental age, constantly encourage and praise, never discourage or scold; and keep them happy. 



\section{APPENDIX}

\section{THE MECHANISM OF THE EMOTIONS}

BY

\section{Professor Angeto Mosso}

Todar I may be permitted to express my own ideas about the mechanism of the emotions.

We are sometimes surprised by a sad or a joyful piece of news. We all know what happens in a state of fear and distress. Physiological phenomena occur that cannot be described. But when we learn suddenly that the news which has troubled us is false, that our fear and distress had no foundation, the internal disturbance does not cease, the physiological phenomena continue in the organism in spite of all efforts of the will to suppress them.

The investigation of these processes has shown that the seat of the emotions lies in the sympathetic nervous system.

Before we were born, and for a long time after birth, our life was entrusted to the activity of the sympathetic system and the reflex movements derived from the spinal cord. We need not be surprised at this, when we reflect how great an importance nature has attributed to the vegetative and generative life processes in the formation of the organism.

In decisive moments of life, when the emotions are most violent, it is just the sympathetic nervous system that comes into action. The intestines and the smooth muscular fibres contract in order to raise the pressure of the blood, and to utilize the blood better for the brain and the muscles.

The first observations concerning this subject were made by me more than twenty years ago. I was able to see that in sleep a contraction of the blood-vessels always takes place as soon as the sense organs and the skin are stimulated, even when the 


\section{PSYCHOLOGY OF THE NORMAL AND SUBNORMAL}

stimulation is so weak that the subject does not wake up. These changes, which result without our knowledge, form one of the most remarkable arrangements which we ean observe among the perfections of our organization. During the interruption of consciousness our body does not remain helplessly exposed to the influences of the external world, or in danger of becoming the prey of its enemies. Even in sleep a portion of the nerve centres watches over the operation of the external world, and prepares in good time the material conditions for the awaking of consciousness. If we glance back at the unconscious processes which we saw take place in sleep under external influences, we shall see that they are all co-ordinated in correspondence with a final object; they all coincide in favouring the circulation of the blood in the brain, and thereby making it possible that, in case of danger, the organ may awake to full activity.

I do not believe myself far from the truth in maintaining that the totality of the reflex movements to be observed during sleep forms a real defensive apparatus for the organism.

Other investigators have since demonstrated the same thing. Two years later, in 1881, Dr. Pellacani and I found that even very weak sensations caused a contraction of the bladder. These facts had, in general, been already known, for these contractions have become proverbial in connection with fear and other emotional conditions; but no one had previously observed that this organ reacts with such facility to all sense imimpressions that its tonicity changes in consequence of attention and inconsiderable psychic processes.

\section{II}

The organs of the abdomen and the pelvic cavity are just as sensitive to the emotions as the heart. I have studied the movements of the abdominal organs, the stomach, and the rectum. In the smallest emotions movements of the intestines and stomach always occur. 


\section{EMOTIONS}

In movements of the bladder, we must distinguish between active and passive, i.e. between such as are peculiar to the bladder itself, and such as are transferred to it from the diaphragm and from the walls of the abdomen.

In order to investigate with exactness these movements of the bladder itself, I have carried on experiments both on the dog and on woman. I shall first explain the construction of the apparatus employed, and then give an account of the experiments performed.

The instrument made use of was my plethysmograph, which has the advantage of maintaining the pressure constant and of registering the slightest movements of contraction and relaxation of the bladder. ... (Here follows description of the apparatus.)

Another day while we were recording the movements of the bladder, a servant, to whom the dog was much attached, entered the laboratory. Immediately the curve showed an active contraction of the bladder, as may be seen in Fig. 3, at G. In $a b c$ we see passive movements of the bladder, which have become weaker because the respiration is more superficial.

When, shortly before, another person, whom the dog did not know so well, had entered the room, we had also noticed another, but stronger contraction of the bladder. In order to keep the dog quiet, one of us laid his hand on his head. (See P in curve of Fig. 3.) When the hand was taken away, and the servant laid his upon the dog, there occurred again an active, but less marked, contraction of the bladder. As soon as the respiration became more superficial, the passive movements of the bladder became also less distinct.

After these observations, the dog lay with eyes half closed, as if he was about to go to sleep. His tail was touched, and immediately afterward the curve showed an active contraction of the bladder, while-what is noteworthy-the rhythm and depth of the respiratory movements did not change. After the bladder had again assumed its full volume, and while the dog was perfectly quiet, his skin was touched, and the curve record 


\section{PSYCHOLOGY OF THE NORMAL AND SUBNORMAL}

showed at once a stronger, active contraction of the bladder. In like manner, sensations of pain, which we produced by pulling the dog's ears, caused strong, active contraction of the bladder.

Such experiments were many times repeated. They were also earried on with bitches, the bladder being directly connected with the plethysmograph by the introduction of a catheter, without previous establishment of a fistula. The results which we obtained were always the same. It was sufficient to speak kindly to the animals, or to earess them, to make the curve express the psychic influence upon their active movements.

But I could not rest content with these results obtained from animals. I needed to corroborate them by experiments on human beings. Naturally this can be done better with woman, since with her the bladder can be easily brought into connection with the plethysmograph by the introduction of a eatheter. My clinical colleagues were kind enough to place at my disposal some girls from the hospitals, who readily offered themselves for the purposes of these experiments.

I may be permitted to give an account of these experiments also. These experiments were carried on, otherwise, as the first. Again I had the thoracic and abdominal respiration and the movements of the bladder independently recorded. The subject lay comfortably on a bed. Here, in state of complete rest, the whole curve of the bladder was, at times, horizontal, showing, at others, slight active undulations. To touch the hand of the subject lightly sufficed, however, to produce at once an active contraction of the bladder. (See $\mathrm{T}$ in the curve of Fig. 4.)

While the subject was lying quietly on the bed, the elockwork of the kymograph was wound up (see $\mathbf{C}$ ). The noise resulting was entirely unknown to the subject, but the impression sufficed to cause itself to be reflected in the bladder, and to induce a contraction, visible in the eurve. When the subject was addressed (see $\mathrm{P}$ ), it could be seen that the bladder contracted immediately, while, if she herself spoke (see R), a series of such contractions took place. All these contractions were move- 


\section{EMOTIONS}

ments proper to the bladder. As was shown by other experiments as well, they were not transferred to the bladder from the abdominal walls as from the diaphragm, and were not, therefore, passive movements $F$. The lower line $T$ marks the seconds.

I was particularly interested in the movements produced in the bladder by purely psychic influences. These are shown, e.g., by the following experiment. While the girl lay quietly on the bed, and respiration was quiet and normal-this is always shown by the curve,--some one said to her, "Now I'm going to pinch you," but without doing so. Immediately the bladder contracted, without the slightest change being noticed in the thoracic and abdominal respiration. After rest had been again restored, a jest was spoken to the girl, and again we perceived a contraction of the bladder on the curve, without seeing any modification whatever of the two respiratory curves.

Beyond all doubt, then, the contractions of the bladder which we observed were movements proper to that organ itself.

All these phenomena may be considered the most delicate reflex movements which occur in the organism. I was particularly interested to know what influence a direct activity of the brain would exercise upon the movements of the bladder, and I carried on experiments to that end. The subject had only a slight education; she was especially a bad mental arithmetician, very easy problems in arithmetic causing her difficulties. She needed, therefore, in such work to exert her brain very much.

While she lay quietly on the bed and her respiration was quite normal, she was given the following example in arithmetic: "How many eggs are seven dozen?" Immediately the bladder was seen to contract (Fig. 5). After this problem was solved (see W), we had her multiply in her head thirteen by twelve, and then a relaxation of the bladder was to be seen.

I noticed, also, that merely speaking to the girl, without her answering, was sufficient to produce a contraction of the bladder. 


\section{PSYCHOLOGY OF THE NORMAL AND SUBNORMAL}

\section{III}

The preponderating activity of the sympathetic system in the emotions is so great that the brain effort is not able to suppress it. Many men feel a contraction in the abdomen when they look down from a tower or other high place. These troublesome sensations, which are connected with the idea of a possible fall, are simply caused by the contraction of the bladder and the intestines.

When we investigate, by means of the plethysmograph, the movements of the intestines during emotional states, we obtain the same curves as we received from the movements of the bloodvessels of the extremities or of the brain, or from the movements of the bladder. All these facts enable us to understand the mechanism of the emotions better. Emotion signifies movement We understand now that the constant and fundamental movements taking place in emotions are the movements of the internal organs of vegetative life.

The investigations earried on in my laboratory by Dr. Kiesow have convinced me that in certain emotions the blood pressure increases, and the blood-vessels and smooth muscular fibres contract in order to prevent the blood from being dammed up in the abdominal cavity.

In order to increase the circulation of the blood in the brain and muscles our bodily machine has to work under a high blood pressure. This end could be attained only thru the sympathetic system, which sends its fibres everywhere to the smooth musculature. During blushing a paling of the skin ean be noticed before the blood-vessels expand, and the blush proper takes place.

In the study of the emotions the reflex movements of the striped musculature of the face, the extremities, and the trunk are of secondary significance to the physiologist. They are simply accompanying phenomena and, just because they are more complicated, less fundamental.

However useful the first reactions of the nervous system are, 


\section{EMOTIONS}

yet we all know that they do not suffice for the defence of the organism in strong emotions. The nerve substance is so irritable that a small shock is enough to disturb the equilibrium. I will not enter into detail here, since I have already shown in my book on Fear, how unstable is the equilibrium of the nervous system, and how easily the brain and the sympathetic system go beyond the proper measure in their activity when danger threatens, and existence is at stake.

Even a practiced observer is often unable to decide from the gestures and facial expression of an individual whether he is enraged or in a state of the greatest joy.

To recall the expressions of two so opposite emotional conditions suffices to convince us that the reflex phenomena accompanying them are not only useless, but even injurious. Indeed, in great pain and great pleasure we have seen the same phenomena; trembling of the muscles, secretion of tears, expansion of the pupils, decrease of visual acuity, buzzing in the ears, oppression of the breathing, palpitation of the heart, inability to speak, exclamations, convulsive movements of the diaphragm, etc. All these phenomena are injurious. After the emotion is over we feel nervous fatigue, have headache, and suffer from insomnia. I am sorry to find myself in this matter in disagreement with Darwin, but I cannot concede that the unconscious processes occurring during the emotions (at least the best known and most characteristic) have always a physiological purpose.

If we compare the expressions of pleasure and satisfaction in their highest degree with those of pain, it will be seen that there is one and the same mechanism for both. In my book on Fear I have shown that it is the quantity and not the quality of the excitation which disturbs the equilibrium of our organism. Only the processes which take place in the system of the great sympathetic are purposive and advantageous for the preservation of life. And it cannot be otherwise. The animals, whose involuntary movements preserved them from destruction in danger, won in the struggle for existence over others who possessed in less marked degree this capacity. 


\section{PSYCHOLOGY OF THE NORMAL AND SUBNORMAL}

Whatever the emotions may be, we always see that in these states the blood pressure increases, the heart beats become stronger, and the respiration deeper. These advantageous effects are the same in man as in animals, when they fix the attention, are passionately excited, curious or jealous, or when they run at play or in pursuit of prey.

But so soon as the emotion becomes more intense, the equilibrium of the organs ceases. The condition of excitability is increased and becomes more complicated, contractions of the muscles and changes in the sense organs take place, from which it results that the capacity for resistance of the organism is lowered. In strong emotions, as in rage and anger, we are overpowered by unconscious and disco-ordinated movements, and a penetrative and irresistible transformation occurs in us, as if the influence of education had been extinguished, as if our reason had suffered an eclipse. We are no longer able to suppress the internal excitement, the voice refuses its office, and we utter a wild cry. Many persons in such states gnash their teeth like wild beasts, others act foolishly, like children.

These disturbances occur not only in the reflex movements, but also in the conscious processes, and more even in the latter than in the former. Education has taught us that we must seek to master and to calm ourselves during this internal excitement, for in these states we lack mental clearness and power of judgment, and consciousness cannot again regain control until these disco-ordinated reflex movements have ceased. Even the ancients knew that strong emotions resembled a suddenly occurring sickness. The legend of ancient Rome idealized a king in order to represent war. They gave him the name Hostilius, which is derived from hostis. Tradition further informs us that this king erected a temple to "Pallor and Fear," for pallor and fear were looked upon as malevolent, destructive deities who must be appeased in order that the soldiers might be victorious in battle.

Reprinted by permission from the Decennial Celebration of Clark University, Worcester, Mass., 1899, pp. 396-407. 


\section{BIBLIOGRAPHY}

1. Anderson, Meta L. Education of Defectives in the Public Schools. Yonkers-on-Hudson, World Book Co., 1917. $104 \mathrm{p}$.

2. Bailey, Frederick R., and Miller, Adam Marion. Textbook of Embryology. New York, Wm. Wood \& Co., 1909. $672 \mathrm{p}$.

3. Barnes, Earl. A Study in Children's Drawings. Stanford University, Studies in Education, 1896-7, vol. 1. Philadelphia, 1902, vol. 2. (See also Ped. Sem., 1893, vol. 2, no. 3, pp. 455-463.)

4. Binet, Alfred. The Psychology of Reasoning. Chicago, Open Court Publishing Co., 1912. 191 p.

5. - and Simon, Th. The Development of Intelligence in Children. Translated by Elizabeth S. Kite. Vineland, The Training School, publication no. 11, 1916. $336 \mathrm{p}$.

6. - and Simon, Th. The Intelligence of the Feebleminded. Translated by Elizabeth S. Kite. Vineland, The Training School, publication no. 12, 1916. $328 \mathrm{p}$.

7. Bolton, J. S. A Contribution to the Localization of Cerebral Function. Brain, 1911, vol. 33, pp. 26-148.

8. Cannon, Walter B. Bodily Changes in Pain, Hunger, Fear and Rage. An account of recent researches into the function of emotional excitement. New York, D. Appleton \& Co., 1915. 311 p.

9. Church, Archibald, and Peterson, Frederick. Nervous and Mental Diseases. Philadelphia, W. B. Saunders Co., 1905. $937 \mathrm{p}$.

10. Crile, George W. Man-an Adaptive Mechanism. New York, Macmillan Co., 1916.387 p. 
11. Crile, George W. The Origin and Nature of the Emotions. Philadelphia, W. B. Saunders Co., 1915. $240 \mathrm{p}$.

12. Doll, Edgar A. Clinical Studies in Feeble-mindedness. Boston, Badger, 1917. $233 \mathrm{p}$.

13. Donaldson, Henry Herbert. Anatomical Observations on the Brain and Several Sense-organs of the Blind Deaf-mute, Laura Dewey Bridgman. Am. Jour. of Psy., 1890, vol. 3, pp. 293-342; 1891, vol. 4, pp. 248294.

14. The Growth of the Brain. A study of the nervous system in relation to education. New York, Chas. Scribner's Sons, 1895. $374 \mathrm{p}$.

15. Dunlap, Knight. An Outline of Psychobiology. Baltimore, Johns Hopkins Press, 1914. 121 p.

16. Flechsig, Paul. Gehirn und Seele. Leipzig, Veit u. Comp., 1896. 112 p.

17. Freeman, Frank Nugent. The Psychology of the Common Branches. Boston, Houghton Mifflin Co., 1916. $275 \mathrm{p}$.

18. Gesell, Arnold L., and Gesell, Beatrice Chandler. The Normal Child and Primary Education. Boston, Ginn $\&$ Co., 1912. $342 \mathrm{p}$.

19. Gilchrist, Edward P. The Extent to Which Praise and Reproof Affect a Pupil's Work. School and Society, 1916, vol. 4, no. 101, pp. 872-874.

20. Goddard, Henry Herbert. The Adaptation Board as a Measure of Intelligence. Tr. Sch. Bull., 1915, vol. 11 , no. 10, pp. 182-188.

20a. - The Criminal Imbecile. New York, Macmillan Co., 1915. $157 \mathrm{p}$.

20b. - Feeble-mindedness: Its Causes and Consequences. New York, Macmillan Co., 1914. 599 p.

20c. A Group of Feeble-minded Children with Special Regard to Their Number Concepts. Supplement to the Tr. Sch., 1908, March, pp. 1-16.

[338] 


\section{BIBLIOGRAPHY}

21. Greenman, M. J. Regeneration of Peripheral Nerves. Jour. of Nerv. and Ment. Dis., 1916, vol. 43, no. 1, pp. 62-68.

22. Hammarberg, Carl. Studien über Klinik und Pathologie der Idiotie nebst Untersuchungen über die normale anatomie der Hirnrinde. Upsala, 1895. 126 p.

23. Herrick, C. Judson. An Introduction to Neurology. Philadelphia, W. B. Saunders Co., 1916. 355 p.

24. Hobhouse, L. T. Mind in Evolution. New York, Macmillan Co., 1901. 415 p.

25. Hollingworth, H. L., and Hollingworth, Leta S. Vocational Psychology. New York, D. Appleton \& Co., 1916. $308 \mathrm{p}$.

26. - and Poffenberger, A. T. Applied Psychology. New York, D. Appleton \& Co., 1917. 337 p.

27. Huey, Edmund B. Backward and Feeble-minded Children. Baltimore, Warwick \& York, 1912. $221 \mathrm{p}$.

28. James, William. Talks to Teachers on Psychology and to Students on Some of Life's Ideals. New York, Henry Holt \& Co., 1904. 301 p.

29. Keller, Helen. The Story of My Life. New York, Doubleday, Page \& Co., $1903.441 \mathrm{p}$.

30. Lapage, C. Paget. Feeble-mindedness in Children of School age. Manchester, England, University Press, 1911. $359 \mathrm{p}$.

31. Lindley, Martha. The Reading Ability of Feeble-minded Children. Tr. Sch. Bull., 1917, vol. 14, pp. 90-94.

32. Loeb, Jacques. The Organism as a Whole. From a Physio-chemical Viewpoint. New York, G. P. Putnam's Sons, 1916. 379 p.

33. Luciani, Luigi. Human Psychology. New York, Macmillan Co., 1915. $667 \mathrm{p}$.

34. McDougall, William. An Introduction to Social Psychology. Boston, John W. Luce \& Co., 1915. 431 p.

35. MeMurrich, J. Playfair. The Development of the Human 


\section{PSYCHOLOGY OF THE NORMAL AND SUBNORMAL}

Body. A manual of human embryology. Philadelphia, P. Blakiston's Sons \& Co., 1903. 527 p.

36. Macnamara, N. C. Instinct and Intelligence. London, Henry Frowde with Hodder \& Stoughton, 1915. $216 \mathrm{p}$.

37. Mateer, Florence. Child Behavior. A critical and experimental study of young children by the method of conditioned reflexes. Boston, Badger, 1918. $239 \mathrm{p}$.

38. Morat, J. P. Physiology of the Nervous System. Chicago, W. T. Keener \& Co., 1906. 680 p.

39. Morgan, C. Lloyd. An Introduction to Comparative Psychology. New York, Chas. Scribner's Sons, 1896. $382 \mathrm{p}$.

40. Mosso, Angelo. Fear. New York, Longmans, Green \& Co., 1896. $278 \mathrm{p}$.

41. - The Mechanism of the Emotions. Worcester, Mass., Clark University Decennial Celebration Volume, 1899. pp. 396-407.

42. Pillsbury, W. B. The Psychology of Reasoning. New York, D. Appleton \& Co., 1910. 306 p.

43. Ribot, Th. Essay on the Creative Imagination. Chicago, Open Court Publishing Co., 1906. 370 p.

44. - The Psychology of the Emotions. New York, Chas. Scribner's Sons, 1897. $455 \mathrm{p}$.

45. Santee, Harris E. Anatomy of the Brain and Spinal Cord with Special Reference to Mechanism and Function. Philadelphia, P. Blakiston's Son \& Co., 1915. 474 p.

46. Shand, Alexander F. The Foundations of Character. London, Eng., Macmillan \& Co., Ltd., 1914. 532 p.

47. Sherlock, E. B. The Feeble-minded. A guide to study and practice. London, Eng., Macmillan \& Co., Ltd., 1911. $327 \mathrm{p}$.

48. Sherrington, Charles S. The Integrative Action of the Nervous System. New York, Chas. Scribner's Sons, 1906. $411 \mathrm{p}$.

49. Shuttleworth, G. E., and Potts, W. A. Mentally Deficient [340] 


\section{BIBLIOGRAPHY}

Children, Their Treatment and Training. Philadelphia, P. Blakiston's Son \& Co., 1910. 236 p.

50. Terman, Lewis M. The Measurement of Intelligence. Boston, Houghton Mifflin Co., 1916. 362 p.

51. Titchener, Edward Bradford. A Primer of Psychology. New York, Macmillan Co., 1900. 316 p.

52. Thorndike, Edward L. The Elements of Psychology. New York, A. G. Seiler, 1905. $351 \mathrm{p}$.

53. Tredgold, A. F. Mental Deficiency (Amentia). London, Eng., Balliere, Tindall \& Cox, 1914. 491 p.

54. Watson, John B. Behavior. An introduction to comparative psychology. New York, Henry Holt \& Co., 1914. $439 \mathrm{p}$.

55. White, William. Outlines of Psychiatry. Jour. of Nerv. and Ment. Dis. Mon. no. 1, New York, 1915. 320 p. 



\section{INDEX}

A

Accident, fear of, 279

Action, automatic, 159

impulsive, 194

instinctive, 193

perfection of, depends upon the neuron pattern, 193

reflex, 193

volitional, 195

Adaptability, a measure of intelligence, 58

Adaptation Board, 102

Admiration, 140, 155

Adrenal gland, 132 secretion, effect of, 138

Affections, the, 123

Age, physiological, 60

Alexia, 178

Ambitious, parents and teachers, 311

Ameboid processes, 29

Anger, 124

Anger and adrenal glands, 134

Animals have language, 174

think, 158

wild, approach if eyes are closed, 48

Antagonism between different parts of the sympathetic, 144

Aphasia, 178 case of, 111

Aphasias, table of simple, 179

Aristocracy, 236

Arrested mental development, 52

limitations of persons of, 243

Artistic sense, impossible to develop, 101

Association areas, 98

necessary for complex emotions, 142

Association areas, to hasten the functioning of the great, 216 poverty of neurons in the, 153

Association centers, 65

higher processes depend upon, 109

last to develop, 67
Association, free, 81

of ideas, 45,83

inherent in the nature of the nerv. ous mechanism, 83

logical, 110

neurons, 12

by similarity, 94

importance of, 99

Attention, 76

acquired, 107

and interest, 136

lack of acquired, characteristic of the feeble-minded, 108

objection to our view, 82

voluntary, 202

Automatic action, 208

in a mature mind, 209

"Avalanche Conduction," 151

Awe, 140, 154

\section{B}

Barnes, Earl, 48

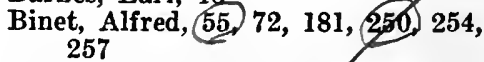

on who is mentally defective, 60

Bladder, contractions of the, 126

Blocking, explanation of, 197

Bolton, 13

Bogardus factory test. 301

Brain of arrested development, 63

Brain of embryo, 7 necessary to thought, 159 patterns, limitation of, 67 weight, 6

Bridgman, Laura, 16 cortex of, 17

Brodmann, 37

Burnet, Thomas, 158

C

Cajal, 151

Cannon, W. B., 126, 128, 138, 144, 228

Cell body, 8 


\section{INDEX}

Charity, recipients of, 246

Child, nature of, 311 without well formed habits, 217

Children, gifted, 216 without reverence, 142

Chinese tailor, 184

Church \& Peterson, 178

Citizenship, good, 262

Crampton, C. Ward, 60

Cretinism, 271

Crile, 126, 228

Criminals, 248

lack of emotion in, 152

Commendation and approval, 321

Concrete, abstract, 283

Conditioned reflex, 147

Conflict, 195

Consciousness, 24

as cause, 83

complex patterns give rise to larger, 76

Consciousness, connected with the passage of neurokyme, 31

cortex is the seat of, 31

marginal, 78

maturity of, 33

the result of interference, 28 seat of, 27

Conscious reflex, 194

Contempt, 154

Contiguity, association by, 84, 92 verbal, 96

Convolutions, 7

Cortex, 7

of Laura Bridgman, 17

neuron layers in, 13

Cowboy, 150

Cretin, 229

Curiosity, 145, 148

leads a child to explore, 91

Curve of distribution, 235

of mental development, 34

\section{D}

Deductive, 183

Defect by deprivation, 57

Defectives never able to handle abstractions, 287

associate by contignity, 87

cannot concentrate, 107

Deliberate, what happens when we, 197
Deliberation, 194

objection to the term, 197

Democracy, 236

Dendrite, 8

Dentist and idiot, 274

Deprivation, defect by, 57

Des Cartes, 140

Diamond, copying the, 255

Differences are perceived before similarities, 101

more easy than likenesses, 294

Discipline, 29.2

among the feeble-minded, 326, 327

fundamental problem of, 316

Diplomacy, honesty in, 290

Disgust, 148

Donaldson, H. H., 16

Drawing, child's first, 48

Dull normal person, 59

\section{E}

Education, 242

and intelligence, 54

Efficiency, 244

total mental, 262

Ego, 197

Emotion, 124

Emotional accompaniment, 196

Emotions, conflict of, 149 complex, 140

complex, result of extensive associations, 141

complex, not much more violent than the simple, 143

distinct from the other phases of mind, 145

intelligence controls the, 272

manifestation of, different in the feeble-minded, 153

nerve action underlying the complex, 142

primary, in the feeble-minded, 145 the mechanism of the, 329

Encephalon, 4, 5

Encouragement, effect of, 318

End organs, 16

Environment to which one can adapt himself, 261

Envy, 155

Experience, 277

child must be given, 291

develops neuron patterns, 48 


\section{INDEX}

Experience (Continued)

fundamental to good judgment, 188

poverty of, 164

school, 263

sets patterns into activity, 288

vicarious, 280

Eye cannot see itself, 207

\section{F}

Factory test, 301

Fascination, 140, 155

Fear, 124

and curiosity antagonistic, 150 paralyzed by, 125, 273

Feeble-minded, 52,57 child steals a handkerchief, 295

dissipation of mental energy of, 161

fond of music, 75

have memory and imagination, 74

have very incomplete neuron patterns, 152

how to train the, 297

in the army, 250

in the high schools, 115

lack neurons for emotions, 155

lacking in reasoning, 188

limitations of the, 102

not hard to please, 274

not able to build up elaborate neuron patterns, 108

Feeble-minded, the great problem, 312

who need to go to an institution, 252

often repeat judgments, 182

not naturally bad, 275

lacking in energy, 189

do not modify their instincts, 147

pedagogy of the, 293

vicarious experience nil, 283

reason for the weak volitional action of. 205

Feeble-mindedness defined, 58

upper limit of, 59

we may suspect, 295

Feeble-minded children cited: Bert, 162; Charles, 191: Eddie, 161, 299; Fire-alarm Joe, 75; Florence, 87; Garry, 163; George, 153; Jay, 184; John, 324;
Feeble-minded children (Continued) John's proticiency in numbers, 283; Kirk, 167; Lewis, 285; Lil, 154; Marjorie B., 74; Robert, 74; Theodore, 190; Tom. 162, 325; Willie, 154

Feeling of familiarity, 71

Feelings, 123, 136

as guides for future action, 316

Elechsig, $15,65,98$

Flight, 145

Foote, Elizabeth, 255, 260

Frog, experiment with, 21

Frequency, 196

\section{G}

Galen, 227

Ganglion. 10 sympathetic, 6 spinal, 20

Generalizing, 183

Geography, teaching, 282

Gilchrist, 318

Glands of internal secretion, 133

Goff, Judge, 253

Gordian knot, 207

Gratitude, 141, 155

Greenman, M. J., 30

\section{$\mathbf{H}$}

Habit, 212

vs. no habit, 217

based on an impulse, 214

an acquired instinct, 212

Habits easily broken, 219

good and bad, 215

of inaction, 201

of the feeble-minded, 222

why we have undesirable, 219

Harum, David. 198

Harvard and Yale, 96

Hauser, Caspar, 48

Hammarberg, 63

Happiness first, 323

Healy, Wm., 269

Heidelberg, 212

Heredity, what is. 241

Herrick, 9, 12, 27, 28, 66, 70, 98, 110

Holmes, Oliver Wendell, 118 


\section{INDEX}

I

Iceberg, figure of, 25

Idea, 45

Ideas, association of, 45,83

Ideal of training, 313

Idiot, 60

a creature of impulse, 265

capable of instinctive attention, 78

mícrocephalic and hydrocephalic, 79

Idiots associate, 114

recite Psalms, 114

repetition of movements, 49

see and hear, 65

Image, a mental, 45

Imagination, 72

creative, 117

not unlimited, 117

possible for undeveloped minds, 74

reproductive, 73

Imbeciles, 60

define in terms of use, 184

few accomplishments of, 50

Impulsive action, 194

action dangerous, 215

habits, 214

Impulse, individual differences of, 265

Inductive, 182

Industrial education, 292

Infant, conscious as born, 32 new born, 40

Inhibitory action, 197

Instinct, 38

an inherited habit, 212

strength of, 275

Instincts according to McDougall, 39

are modified, 146

Intelligence of the average person, 53, (235.) 251 )

and emotion, 270

and training, 247

and will, 264

can be measured, 250

cause of low, 238

confused Fith education, 54

defined, 56

different from education, 238

eight year, etc., 53

level, 60
Intelligence (Continued)

losses come from lack of, 244

measuring, 55,116

of various groups determined, 234 ranges of, 234

twenty year, the maximum, 53, 56

Interest, 136

and attention, 136

Irritability, 69

\section{$J$}

James, Wm., 58, 66, 126, 197, 200, $201,202,203,206,212,215,221$, 262,264

James-Lange theory, 138

James' maxims for habit forming, 218

Jesus, prayer of, 291

Judgment, 167, 178

why the mental defective shows poor, 190

Judgments, reasoning a train of, 182

Junk shop, 279

Juries nonplussed, 152

\section{$\mathbf{K}$}

Keller, Helen, 167

Knee jerk, 20

Knowledge of the "first order," 86

$\mathbf{L}$

Lange, 126

Lange-James theory, 138

Language, 174

Levels of intelligence, 56, 249

Lindley, Martha, 303

Loathing, 140, 155

Localization, brain, 12

Lugaro, 29

Lying and stealing, 289

\section{M}

Man with the Hoe, 239

Markham, Edwin, 239

Mateer, Florence, 147

McDougall, Wm., 123, 155, 140, 146, 149. 290

instinct defined by, 38 


\section{INDEX}

Measuring scales for intellizence, 55, 250,261

Medicine, license to practise, 244

Medullation, 15

Memory, 69, 110

acquired, 110

of the feeble-minded, 75

inherent, 71

idiots show, 75

man losing the power of, 76

poor, 72

a property of nervous tissue, 71

Mental age, 53, 60

Mental, arrest, 52

defect, wisest procedure to suspect, 313

image, 73

levels, 233, 246

Mental levels depend upon the time when arrest occurs, 64

determined with much accuracy, 250

educate according to, 253

theory of, 235

Mental tests in the army, 251

the theory of, 254

Mesial surfaces, 7

Messenger boy, 246

Metric system remembered, 115

Microcephaly, 63

Microcephalic idiot, $\mathbf{7 9}$

Millet, 239

Mind, apathy of undeveloped, 151

two phases of, 271

Mongolian type, 186

Moral imbecile, 267

Moral training, 310

Morgan, Lloyd, 156

Moron, defined, 58

a menace, 237

the discovery of the, 233

develops some neuron patterns, 50

a problem of education, 252

Morons, 60

apparently normal until 7 or 8 years, 61

brains of, 64

not vicious, 275

who had run away, 163

inability to hold a job, 205

Mosso, 126, 138, 228, 270

Motive, 315

Movement of the heart arrested, 197
Murderers studied by the writer, 152

Muscle, goal of neurokyme, 16

$\mathbf{N}$

Name, recall of a, 81

Names, what are, 174

Naming instinct, 176

Natural retentiveness, 71

Nerve action underlying the com. plex emotions, 142

degeneration in, 30

energy dissipated, 161

Nervous system, 3

the autonomic, 130

properties inherent in, 69

Neurite, 8

Neurokyme, 9, 20

is blocked, 92

Neuron action and sympathetic system, 127

development at sixteen years, 54

paths must be blocked, 291

Neuron pattern, 21

and association by similarity, 95

in instincts, 142

elaborateness of, 195

inductive reasoning a question of, 185

simple, 35

simplest kind of cortical, 165

very complicated, 36

Neuron patterns, 23

acquired, 39,42

built up by experience, 48, 151

elaborated by experience, 45

innate, 38

increasing in complexity, 48

patterns, man a slave to his, 207

in the feeble-minded, 49

specific, 37

Neurons, 8

at birth, 15

changed permanently, 70

condition of, in the feeble-minded, 62

dependent upon stimulation, 16

develop until forty-five, 33

grow at different rates, 14

impossible to develop the defective, 97

in contact, 29 


\section{INDEX}

Neurons (Continued)

layers of the cortex, 13

motor, 15

sensory, 15

Norsworthy, Naomi, 87

Number work of feeble-minded children, 308

\section{0}

Over inhibited type, 200

\section{$\mathbf{P}$}

Parental instinct, 145

Patriotism, 154

Pathological liars, 269

Pavlov, 131

Penmanship of the feeble-minded, 308

Perception, 44, 167, 171, definition, 181

sometimes called judgment, 178 and judgment differ only in degree, 181

judgment and reasoning the same process, 188

Peterson, Church and, 178

Pity, 155

Pleasure, feeling of, 124

Pneumogastric nerve, 197

Porteus tests, Lewis and the, 285

Pugnacity, 145, 149

Punishment, 318

vs. brutality, 319

Punning, 118

\section{$\mathbf{Q}$}

Quickening, 32, 33

\section{$\mathbf{R}$}

Rage and adrenal glands, 134

blind with, 125

Rami communicantes, 127

Reading, an investigation of, 303

Reasoning, 182

course of nerve energy in deductive, 186

deductive, 183

dependent upon experience, 183

inductive, 183
Recency, 196

Relay, 23

Reflex-arc, 25

Reflex, patella, 20

Reflexes, neuron patterns for, 38

Reproach, 154

Repulsion, 145

Resentment, 155

Responsibility, ideas of, 243

Retina, 16

Reverence, 141

no evidence of, among the feebleminded, 153

Rewards or punishments, 317

Reward must be definite, 320

Rousseau, 212

Rugh, $17 \%$

$\mathbf{s}$

Scorn, 140, 154

Secret of will, 207

Self-abasement, 145, 149

Self-assertion, 145, 149

Self-made men, 242

Sensation, 41

adults seldom have pure, 165

is consciousness of a stimulus, 165 of pressure, 172

of sound, 71

Sex emotion, 273

Sheath, medullary, 15

Similarities, children do not easily see, 101

Similarity, association by, 94 degrees of, 99

Snakes, imbeciles and idiots do not fear, 148

Society, a perfect, 243

Sound-sight, 166

Splanchnic nerves, 131

Square, copying the, 255

State license, 244

Steel, heated and cooled, 70

Stern, 58

St. Lawrence River, 282

Stout, 178

Stimulus, 16 single, tends to radiate in all directions, 42

Stimuli do not come singly, 42

infinite number of, 41

interference of two or more, 91 


\section{INDEX}

Stimuli, specific, 16

summation of, 43

Subnormals, training for, 300

Sullivan, Miss, 170

Swiss guide, 150

Syllogism, 185

Symbols, 282

Sympathy, 146

Sympathetic system, 6

the seat of the emotions, 126

the oldest of all nervous structures, 127

Synapse, 10

Telegraphy, 22

Temperament, 226

Temperaments, four, 227 of the feeble-minded, 229

Terman 53, 56, 235

Tests, pedagogical, indication of the mental level, 263

what is tested by each question, 259

Thorndike, 40

Thought, 158, 163 poverty of, 164 results when the action is impeded, 160

Thumb, opposing, to fingers, 260

Thyroid gland, 271

Time, an important point, 202 ability to appreciate, 257

Titchener, 45, 171, 178, 227

Touch, sense of, 16

Train, impulse to jump on, 194

Tredgold defines feeble-mindedness, 58

Truart, the, 292

\section{$\mathbf{U}$}

United States Army, use of mental tests in, 250

\section{V}

Vengeance, 154

Verbal associations, 177

description, 281

memory, $17 \tau$

types, 177

Vicarious experience when valuable, 280

Vineland Training School, child in, 61

Virtue is its own reward, 321

Visual center, 16

Vladivostok, 113

Volitional action, 194, 201 emotional content of, 204

Voluntary habits, 214

W

Watch, winding, 159 automatic, 164

White, Wm. A., 249

Will, the elements of, 201 breaking defended, 264, 267

a matter of the neuron patterns, 201

strong or weak, 203

the way to control, 265

relations of, to intelligence, 264

lacking in feeble-minded, 205

Wilson, President, 289

Wit, 118

Word blindness, 178 deafness, 178 trying to think of a, 82

Words arouse experiences, 177 are only symbols, 177

Writing of the feeble-minded, 308 
7334 
. 




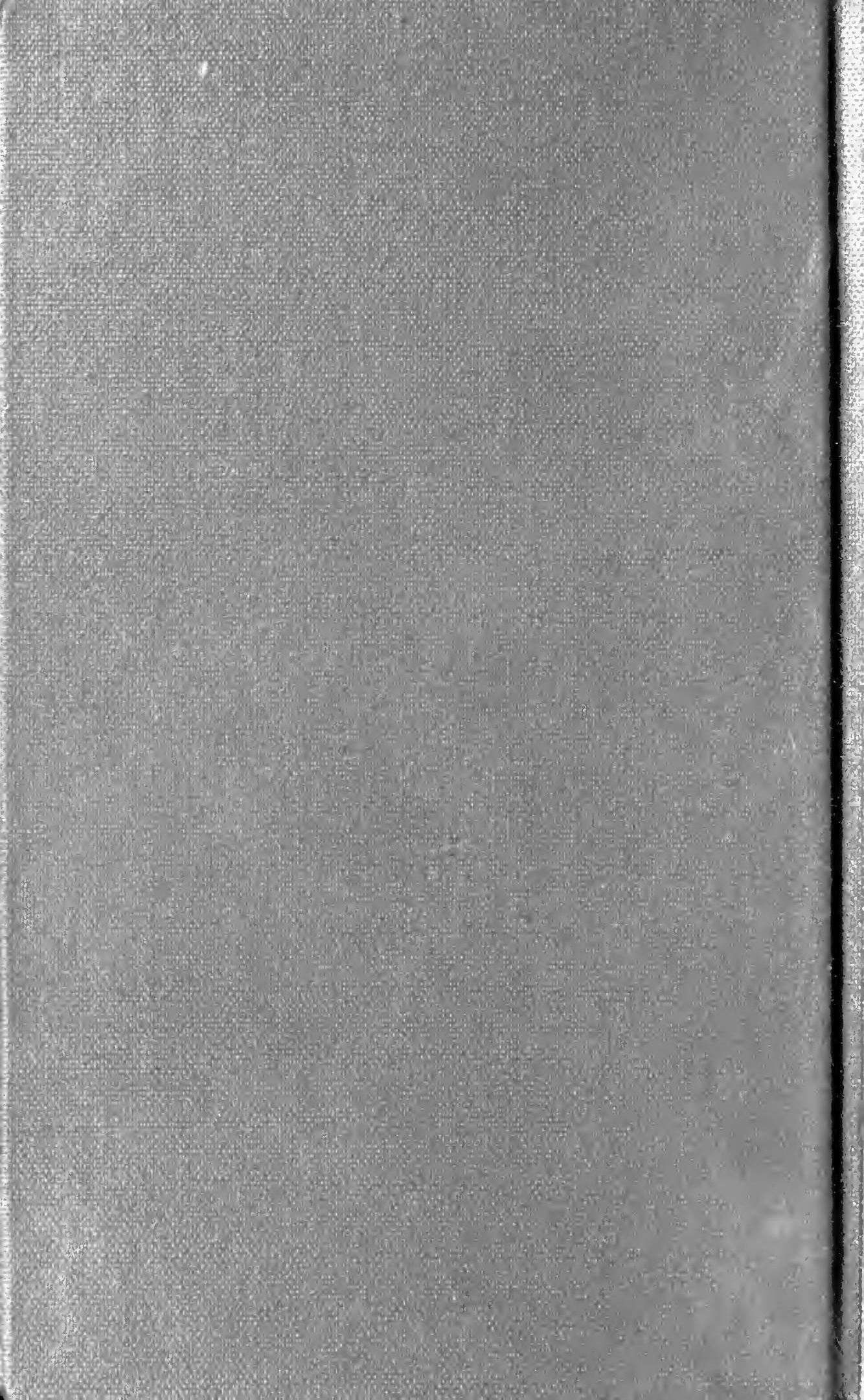

